\title{
A Study of Charged Current Single Charged Pion Productions on Carbon in a Few-GeV Neutrino Beam
}

\section{Katsuki Hiraide}

Department of Physics, Graduate School of Science Kyoto University

January, 2009

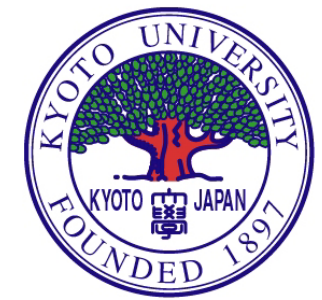





\title{
A Study of Charged Current Single Charged Pion Productions on Carbon in a Few-GeV Neutrino Beam
}

\author{
A dissertation \\ submitted in partial fulfillment of the requirements \\ for the Degree of Doctor of Science \\ in the Graduate School of Science, Kyoto University
}

\section{Katsuki Hiraide}

Department of Physics, Graduate School of Science

Kyoto University

January, 2009 
Dissertation Committee:

\author{
Tsuyoshi Nakaya \\ Noboru Sasao \\ Masashi Yokoyama \\ Tomofumi Nagae \\ Yoshikazu Fujiwara
}




\begin{abstract}
Understanding single charged pion production via neutrino-nucleus charged current interaction in the neutrino energy region of a few $\mathrm{GeV}$ is essential for future neutrino oscillation experiments since this process is a dominant background for $\nu_{\mu} \rightarrow \nu_{x}$ oscillation measurements. There are two contributions to this process: single pion production via baryonic resonance $\left(\nu_{\mu} N \rightarrow \mu^{-} N \pi^{+}\right)$and coherent pion production interacting with the entire nucleus $\left(\nu_{\mu} A \rightarrow \mu^{-} A \pi^{+}\right)$, where $N$ is nucleon in the nucleus and $A$ is the nucleus.

The purpose of the study presented in this thesis is a precise measurement of charged current single charged pion productions, resonant and coherent pion productions, with a good final state separation in the neutrino energy region of a few $\mathrm{GeV}$. In this thesis, we focus on the study of charged current coherent pion production from muon neutrinos scattering on carbon, $\nu_{\mu}{ }^{12} \mathrm{C} \rightarrow \mu^{-12} \mathrm{C} \pi^{+}$, in the SciBooNE experiment. This is motivated by the fact that without measuring this component first, the precise determination of resonant pion production cross section can not be achieved since the contribution of coherent pion production in the region of small muon scattering angle is not small. Furthermore, the coherent process is particularly interesting because it is deeply rooted in fundamental physics via Adler's partially conserved axial-vector current theorem. We took data from June 2007 until August 2008, in both the neutrino and antineutrino beam. In total, $2.52 \times 10^{20}$ protons on target were collected.

We have performed a search for charged current coherent pion production by using SciBooNE's full neutrino data set, corresponding to $0.99 \times 10^{20}$ protons on target. No evidence for coherent pion production is observed. We set $90 \%$ confidence level upper limits on the cross section ratio of charged current coherent pion production to the total charged current cross section at $0.67 \times 10^{-2}$ at mean neutrino energy $1.1 \mathrm{GeV}$ and $1.36 \times$ $10^{-2}$ at mean neutrino energy $2.2 \mathrm{GeV}$. We reveal that the Rein-Sehgal model widely used in neutrino oscillation experiments breaks down at the neutrino energy region of a few $\mathrm{GeV}$. This creates active controversies on the model of coherent pion production, and the understanding of coherent pion production is being progressed.

In addition, future prospects of measurements of charged current single charged pion production in SciBooNE are discussed.
\end{abstract}





\section{Acknowledgements}

First of all, I would like to sincerely express my great appreciation to my supervisor Prof. Tsuyoshi Nakaya for giving me an opportunity for this research. His unerring judgements as a co-spokesperson of SciBooNE led the experiment toward a success. I am grateful for his encouragement, criticism, and honest advice. I would like to thank Prof. Koichiro Nishikawa, my former adviser, who involved me with a series of the exciting neutrino experiments: K2K, T2K, and Super-Kamiokande. I was impressed by his energetic attitude to these experiments and by his leadership. I am thankful to Dr. Masashi Yokoyama for giving me shrewd advice concerning the detector operation and physics analysis. I sincerely express my gratitude to Prof. Yoji Totsuka who helped us to start this experiment.

I would like to acknowledge all the people in the SciBooNE Collaboration. Especially, I would like to give my special thanks to Dr. Morgan Wascko who welded together a team of collaborators as a co-spokesperson of SciBooNE and made several traditions (SciBooNE social club, formal Friday, etc.). I am really glad to have been able to work with him from the beginning of the SciBooNE experiment. I am grateful to Dr. R. Tesarek for leading us as a project manager. I would like express my thanks to Dr. H.-K. Tanaka who is a real axis of SciBooNE. The experiment could not have succeeded without his enormous efforts. I am thankful to Dr. L. Ludovici and Dr. R. Napora for leading the construction and commissioning works for the EC and MRD, respectively. I would like to thank Dr. M. Sorel, Dr. G. Zeller, Dr. S. Brice, Prof. Y. Hayato, and Prof. M. Shaevitz for giving me appropriate advice on my analysis. I am grateful to Dr. T. Katori and K. Mahn for competing with each other for graduation and arranging several social events for us. I really thank Y. Kurimoto, Y. Nakajima, H. Takei, G. Mitsuka, J. Walding, Dr. C. Mariani, C. Giganti, J. Alcaraz, J. Catala, A. Hanson, and G. Cheng for working hard and drinking a lot with me. I spent precious days with them during two years' stay at FNAL, and without them the experiment could not have been achieved in such a short time scale. I am thankful to K. Matsuoka, H. Kubo, D. Orme, Y. Kobayashi, S. Masuike, and S. Mizugashira for helping us construct and/or de-commission the detector.

I would also like to express my thanks to people outside the collaboration who supported our experiment. I would like to thank J. Kubota, Y. Kurosawa, T. Nobuhara, M. Taguchi, Dr. Y. Takenaga, C. Ishihara, T. Koike, and Y. Maruyama for helping us dismantle fibers from SciBar at KEK. I wish to thank M. Taino, K. Hayashi, and all the people from Futaba Kogyo Co., Ltd. and Hitachi Kenki Logistics Technology Co., Ltd. for technical support during disassembly of the SciBar and EC detectors at KEK. I am grateful to American Overseas Transport Ltd. for the safe transportation of SciBar and the EC from KEK to FNAL. I would like to acknowledge engineering supports from E. Chi, E. Villegas, and R. Schmitt, and technical support from J. Voirin, J. Cornele, 
M. Shoun, and B. Lambin during the construction of the SciBooNE detector at FNAL. I am thankful to J. Krider, B. Pritchard, and D. Butler for technical support during testing fibers in Lab 3 at FNAL. I would like to thank H. Kawamuko, S. Gomi, T. Usuki, N. Kobayashi, K. Sugiya, Y. J. Lee, J. E. Jung, and C. S. Moon for helping us install fibers into SciBar at FNAL. I gratefully thank people in Accelerator Division at FNAL for providing us a proton beam in good condition throughout the data-taking period. I am thankful to the MiniBooNE Collaboration for sharing not only neutrino beam but also experimental shifts. I deeply thank M. Otani and A. Murakami for helping us de-commisson the detector.

I am grateful to S. Kunori and K. Kunori for inviting us to plenty of house parties during our stay at FNAL. Every time, we ate and drank a lot, and spent delightful time.

I wish to extend my thanks to the $\mathrm{K} 2 \mathrm{~K}$ and $\mathrm{T} 2 \mathrm{~K}$ Collaborations, the first experiments which I joined. Especially, I would like to thank members of the K2K-SciBar group: Dr. M. Yoshida, Dr. K. Nitta, Dr. H. Maesaka, Dr. M. Hasegawa, Dr. S. Yamamoto, Dr. Y. Takubo, T. Morita, and T. Sasaki. I am also thankful to Prof. T. Kobayashi, Prof. T. Ishii, Prof. A. K. Ichikawa, Dr. T. Ishida, Dr. S. Tada, Dr. T. Nakadaira, Dr. T. Sekiguchi, Dr. I. Kato, K. Hayashi, and S. Ueda.

I am thankful to members of High Energy Physics Group in Kyoto University: Prof. N. Sasao, Prof. T. Nomura, Dr. H. Nanjo, M. Suehiro, Dr. S. Nishida, H. Yokoyama, Dr. Y. Honda, Dr. K. Mizouchi, Dr. T. Sumida, Dr. A. Minamino, H. Morii, Dr. N. Taniguchi, T. Shirai, K. Takezawa, K. Ezawa, K. Shiomi, N. Kawasaki, N. Nagai, T. Masuda, K. Ieki, D. Naito, and Y. Maeda. I would like to express my thanks to A. Nakao, K. Nakagawa, M. Hiraoka, and the other secretaries of Kyoto University for taking care of every business.

I would like to acknowledge supports from the Japan Society for Promotion of Science (JSPS), the Japan/U.S. Cooperation Program in the field of High Energy Physics, Fermi National Accelerator Laboratory, and the global COE program "The Next Generation of Physics, Spun from Universality and Emergence". This work was supported from the MEXT and JSPS (Japan), the INFN (Italy), the Ministry of Science and Innovation and CSIC (Spain), the STFC (UK), and the DOE and NSF (USA).

Finally, I would like to express my special appreciation to my parents for various supports throughout my life.

Katsuki Hiraide Kyoto, Japan January 2009 


\section{Contents}

Acknowledgments $\quad$ i

1 Introduction $\quad 1$

1.1 Neutrinos and neutrino oscillations . . . . . . . . . . . . . . . 1

1.1.1 Neutrinos and their masses . . . . . . . . . . . . . . . . . . 1

1.1.2 Phenomenology of neutrino oscillations . . . . . . . . . . . . . 1

1.1.3 Summary of neutrino oscillation measurements . . . . . . . . . . . 3

1.1.4 Future neutrino oscillation experiment . . . . . . . . . . . . . 4

1.2 Neutrino-nucleus interactions . . . . . . . . . . . . . . . . 4

1.2 .1 Overview ............................. 4

1.2.2 Importance of neutrino-nucleus cross section measurements . . . . . 5

1.2.3 Single pion production . . . . . . . . . . . . . . 8

1.3 Overview of this thesis . . . . . . . . . . . . . . . 13

2 SciBooNE Experiment $\quad 15$

2.1 Overview of SciBooNE . . . . . . . . . . . . . . . . . 15

2.2 History of SciBooNE . . . . . . . . . . . . . . . . . . . 16

2.3 Physics motivations of SciBooNE . . . . . . . . . . . . . . . 17

2.3.1 Precise measurements of neutrino-nucleus cross sections . . . . . . . 17

2.3.2 Measurements of antineutrino-nucleus cross sections . . . . . . . . . 17

2.3.3 Neutrino flux measurements . . . . . . . . . . . . . . . . . 18

3 Booster Neutrino Beam $\quad 19$

3.1 Beamline description . . . . . . . . . . . . . . . . . . . . . . 19

3.1.1 Primary proton beam . . . . . . . . . . . . . . . . 19

3.1.2 Target and magnetic focusing horn . . . . . . . . . . . . . . . . 19

3.1.3 Decay region and absorber . . . . . . . . . . . . . . 20

3.2 Neutrino flux prediction . . . . . . . . . . . . . . . . 20

3.2.1 Simulation of meson productions . . . . . . . . . . . 20

3.2.2 Simulation of meson decays . . . . . . . . . . . . . . . . . 22

3.2.3 Neutrino flux prediction at SciBooNE . . . . . . . . . . . . . . . 22

3.2.4 Systematic uncertainties in the neutrino flux prediction . . . . . . . 23

4 Neutrino Interaction Simulation (NEUT) 25

4.1 Neutrino-nucleus interactions . . . . . . . . . . . . . . . . 25

4.1.1 Quasi-elastic scattering . . . . . . . . . . . . . 25

4.1.2 Single meson production via baryon resonances . . . . . . . . . . 28 
4.1.3 Coherent pion production . . . . . . . . . . . . . 28

4.1.4 Deep inelastic scattering (DIS) . . . . . . . . . . . . . . 31

4.2 Intra-nuclear interactions . . . . . . . . . . . . . . . . . . 32

4.2 .1 Pion interactions . . . . . . . . . . . . . . . . . 32

4.2 .2 Nucleon re-scattering . . . . . . . . . . . . . . . . . . 32

5 SciBooNE Detector 33

5.1 Scintillator Bar Tracker (SciBar) . . . . . . . . . . . . . . . . 34

5.1 .1 Extruded scintillator . . . . . . . . . . . . . 36

5.1 .2 Wavelength shifting (WLS) fiber . . . . . . . . . . . . 36

5.1.3 64-channel multi-anode PMT (MA-PMT) . . . . . . . . . . . . 38

5.1 .4 Readout electronics . . . . . . . . . . . . . . . . . . 39

5.1.5 Gain monitoring system . . . . . . . . . . . . . . . . . . . 40

5.1.6 Energy scale calibration . . . . . . . . . . . . . . . . . . 42

5.2 Electromagnetic Calorimeter (EC) . . . . . . . . . . . . . . . . . . . . 42

5.3 Muon Range Detector (MRD) . . . . . . . . . . . . . . . . . 43

5.4 Data Acquisition (DAQ) System . . . . . . . . . . . . . . . . . . . . . . . . . . 44

5.5 Detector Coordinate and Alignment . . . . . . . . . . . . . . 45

5.5 .1 Detector coordinate . . . . . . . . . . . . 45

5.5 .2 Detector alignment . . . . . . . . . . . . . . . 46

5.6 Detector simulation . . . . . . . . . . . . . . . . . 47

5.6.1 Simulation of detector responses . . . . . . . . . . . . . . . . 47

5.6 .2 Simulation of pion interaction in detector . . . . . . . . . . 48

6 Data Summary $\quad 50$

6.1 Data quality cuts . . . . . . . . . . . . . . . . 50

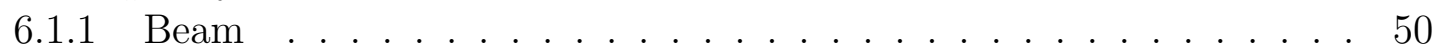

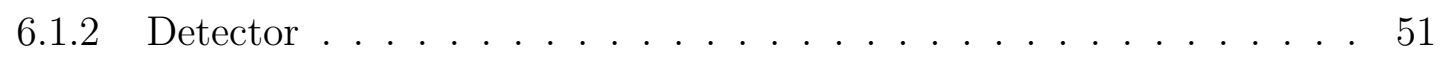

6.2 Summary of data-taking . . . . . . . . . . . . . . . . . . . . . . . . . . . . . . . . . 53

6.2.1 Number of protons on target . . . . . . . . . . . . . 53

6.2.2 Neutrino event rate stability . . . . . . . . . . . . . . . . . . 54

7 Study of Charged Current Coherent Pion Production $\quad 56$

7.1 Analysis overview . . . . . . . . . . . . . . . . . 56

7.2 Charged current event selection . . . . . . . . . . . . . . 56

7.2 .1 1st layer veto . . . . . . . . . . . . . . . . . 57

7.2 .2 Fiducial volume cut . . . . . . . . . . . . . . . . . . . 57

7.2 .3 Event timing cut . . . . . . . . . . . . . . . . . 58

7.2.4 Summary of the SciBar-MRD matched sample . . . . . . . . . . . 60

7.2.5 MRD stopped and MRD penetrated samples . . . . . . . . . . . 60

7.3 Event classification . . . . . . . . . . . . . . . . . . 67

7.3.1 Number of vertex-matched tracks . . . . . . . . . . . . . . . 67

7.3.2 Particle identification . . . . . . . . . . . . . . . . . 67

7.3 .3 Vertex activity . . . . . . . . . . . . . . . 70

7.4 MC tuning . . . . . . . . . . . . . . . . . . . . . 71

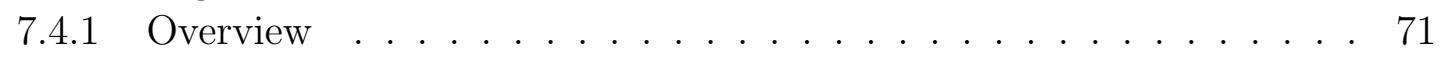

7.4 .2 Fitting parameters ....................... 72 
7.4.3 Definition of $\chi^{2} \ldots \ldots \ldots \ldots \ldots$. . . . . . . . . . . . . . . . . . . . . . . . . . 74

7.4 .4 Covariance matrix . . . . . . . . . . . . . . . . . 75

7.4 .5 Fitting results . . . . . . . . . . . . . . 76

7.5 Coherent pion event extraction . . . . . . . . . . . . . 77

7.5.1 MRD stopped charged current coherent pion events . . . . . . . . 78

7.5.2 MRD penetrated charged current coherent pion events . . . . . . . 82

$7.6 \sigma(\mathrm{CC}$ coherent $\pi) / \sigma(\mathrm{CC})$ cross section ratio $\ldots \ldots \ldots \ldots \ldots . \ldots . \ldots . \ldots . \ldots$

7.6.1 MRD stopped sample . . . . . . . . . . . . . . . 84

7.6.2 MRD penetrated sample . . . . . . . . . . . . . 86

7.7 Systematic errors . . . . . . . . . . . . . . . . . . . . . 87

7.7.1 Detector response and track reconstruction . . . . . . . . . . . 88

7.7 .2 Nuclear effects . . . . . . . . . . . . . . . . . . . . . . . 89

7.7.3 Neutrino interaction models . . . . . . . . . . . . . . . . . . . . 89

7.7 .4 Neutrino beam . . . . . . . . . . . . . . . . . . . 91

7.7.5 Event selection . . . . . . . . . . . . . . . . 92

7.8 Summary and discussion . . . . . . . . . . . . . . . . . . . . . . . . 92

8 Discussion on Future Prospects $\quad 96$

8.1 Coherent pion production . . . . . . . . . . . . 96

8.1.1 Further study of neutrino coherent pion production . . . . . . . . 96

8.1.2 Antineutrino coherent pion production . . . . . . . . . . . 101

8.1.3 Neutral current coherent pion production . . . . . . . . . . . . . . . 101

8.2 Resonant pion production . . . . . . . . . . . . . . . 103

9 Conclusions $\quad 106$

$\begin{array}{ll}\text { A Track Reconstruction } & 108\end{array}$

A.1 Hit preparation in SciBar . . . . . . . . . . . . . . . . . . 108

A.1.1 Crosstalk correction . . . . . . . . . . . . . . . . . 108

A.1.2 Hit threshold for tracking . . . . . . . . . . . . . . . . . 109

A.2 Track reconstruction in SciBar . . . . . . . . . . . . . . . . . 109

A.2.1 2D track reconstruction . . . . . . . . . . . . . . 109

A.2.2 3D track reconstruction . . . . . . . . . . . . . . . 111

A.2.3 Track edge correction . . . . . . . . . . . . . . . . . . 111

A.3 Track reconstruction improvement . . . . . . . . . . . . . . . . . . . . 113

A.3.1 Kink track reconstruction . . . . . . . . . . . . . . . . . . 113

A.3.2 Overlapping track reconstruction . . . . . . . . . . . . . . 115

A.3.3 Reconstruction performance . . . . . . . . . . . . . . 116

A.4 Matching between SciBar and MRD tracks . . . . . . . . . . . . 118

A.4.1 Track-based matching . . . . . . . . . . . . . . . . . 118

A.4.2 Hit-based matching . . . . . . . . . . . . . . . . . . 119

B Low $Q^{2}$ Data Excess in the $\mu+p$ Sample $\quad 120$

C Kinematic Distributions after the MC Tuning 123

$\begin{array}{ll}\text { List of Tables } & 123\end{array}$ 


\section{Chapter 1}

\section{Introduction}

\subsection{Neutrinos and neutrino oscillations}

\subsubsection{Neutrinos and their masses}

Neutrino is a neutral lepton with a very tiny mass, which was originally postulated by Pauli in 1930 in order to explain the continuum electron energy spectrum from the $\beta$ decay. There exists three types of neutrinos: electron neutrino $\left(\nu_{e}\right)$, muon neutrino $\left(\nu_{\mu}\right)$, and tau neutrino $\left(\nu_{\tau}\right)$. The possibility of the existence of the fourth light neutrino $\left(m_{\nu}<M_{Z} / 2\right)$ was excluded by the measurement of the invisible decay width of $Z$ boson in the LEP experiments.

Neutrino masses have been searched for by the direct kinematic measurements, neutrinoless double-beta decay searches, and neutrino oscillation experiments. In addition, another constraint on neutrino masses comes from cosmology. No evidence for finite neutrino masses is obtained from the direct kinematic measurements, neutrino-less double-beta decay searches, and cosmology until now, except from the neutrino oscillation experiments.

\subsubsection{Phenomenology of neutrino oscillations}

In general, a flavor eigenstate of neutrino, $\left|\nu_{\alpha}\right\rangle(\alpha=e, \mu, \tau)$, can be expressed as a superposition of mass eigenstates, $\left|\nu_{i}\right\rangle(i=1,2,3)$;

$$
\left|\nu_{\alpha}\right\rangle=\sum_{i} U_{\alpha i}\left|\nu_{i}\right\rangle
$$

where $U_{\alpha i}$ is an element of a $3 \times 3$ unitary matrix $U$ which is referred to as Maki-NakagawaSakata (MNS) matrix [1]. The matrix is expressed using four independent parameters: three mixing angles, $\theta_{12}, \theta_{23}$, and $\theta_{13}$, and one $\mathrm{CP}$ phase $\delta$;

$$
\begin{aligned}
U & =\left(\begin{array}{ccc}
1 & 0 & 0 \\
0 & c_{23} & s_{23} \\
0 & -s_{23} & c_{23}
\end{array}\right)\left(\begin{array}{ccc}
c_{13} & 0 & s_{13} e^{-i \delta} \\
0 & 1 & 0 \\
-s_{13} e^{i \delta} & 0 & c_{13}
\end{array}\right)\left(\begin{array}{ccc}
c_{12} & s_{12} & 0 \\
-s_{12} & c_{12} & 0 \\
0 & 0 & 1
\end{array}\right) \\
& =\left(\begin{array}{ccc}
c_{12} c_{13} & s_{12} c_{13} & s_{13} e^{-i \delta} \\
-s_{12} c_{23}-c_{12} s_{13} s_{23} e^{i \delta} & c_{12} c_{23}-s_{12} s_{13} s_{23} e^{i \delta} & c_{13} s_{23} \\
s_{12} s_{23}-c_{12} s_{13} c_{23} e^{i \delta} & -c_{12} s_{23}-s_{12} s_{13} c_{23} e^{i \delta} & c_{13} c_{23}
\end{array}\right),
\end{aligned}
$$

where $c_{i j}=\cos \theta_{i j}$ and $s_{i j}=\sin \theta_{i j}$. 
Generated as $\nu_{\alpha}$, the state of neutrino at time $t$ after traveling distance $L$ is expressed as

$$
|\nu(t)\rangle=\sum_{i} U_{\alpha i} e^{-i\left(E_{i} t-p_{i} L\right)}\left|\nu_{i}\right\rangle
$$

where $E_{i}$ and $p_{i}$ are the energy and momentum of $\nu_{i}$ in the laboratory frame, respectively. In practice, neutrino is extremely relativistic due to the tininess of the mass, and thus we can make the following replacements:

$$
\begin{aligned}
& t \sim L, \\
& E_{i}=\sqrt{p_{i}^{2}+m_{i}^{2}} \sim p_{i}+\frac{m_{i}^{2}}{2 p_{i}} .
\end{aligned}
$$

Since $\nu_{\alpha}$ is produced with a definite momentum $p$, all of $\nu_{\alpha}$ 's mass eigenstates have a common momentum. Thus, the probability $P\left(\nu_{\alpha} \rightarrow \nu_{\beta}\right)$ that $\nu_{\beta}$ is observed after $\nu_{\alpha}$ travels the distance $L$ is given by

$$
\begin{aligned}
P\left(\nu_{\alpha} \rightarrow \nu_{\beta}\right)= & \left|<\nu_{\beta}\right| \nu(t)>\left.\right|^{2}=\left|\sum_{i} U_{\alpha i} U_{\beta i}^{*} e^{-i p L} e^{-i \frac{m_{i}^{2} L}{2 p}}\right|^{2} \\
= & \delta_{\alpha \beta}-4 \sum_{i>j} \operatorname{Re}\left(U_{\alpha i} U_{\beta i}^{*} U_{\alpha j}^{*} U_{\beta j}\right) \sin ^{2} \frac{\Delta m_{i j}^{2} L}{4 E} \\
& -2 \sum_{i>j} \operatorname{Im}\left(U_{\alpha i} U_{\beta i}^{*} U_{\alpha j}^{*} U_{\beta j}\right) \sin \frac{\Delta m_{i j}^{2} L}{2 E},
\end{aligned}
$$

where $\Delta m_{i j}^{2} \equiv m_{i}^{2}-m_{j}^{2}$ is the mass squared difference between $\nu_{i}$ and $\nu_{j}$. The sign of the last term in Equation 1.6 is + instead of - in the case of the expression for antineutrinos. Because of the condition $\Delta m_{12}^{2}+\Delta m_{23}^{2}+\Delta m_{31}^{2}=0$ to be imposed, the number of independent parameters for neutrino oscillations is six in the case of three lepton generations: three mixing angles, $\left(\theta_{12}, \theta_{23}, \theta_{13}\right)$, one CP phase, $\delta$, and any two out of three mass squared differences, $\Delta m^{2}$ 's.

From the past experimental results, it is known that the mass squared difference $\Delta m_{12}^{2}$ is small compared with the other two: $\Delta m_{23}^{2} \simeq \Delta m_{31}^{2} \gg \Delta m_{12}^{2}$. In this case, when we focus an attention on the neutrino energy region $E \sim\left(\Delta m_{23}^{2} \cdot L\right)$ which is interesting in the long-baseline neutrino oscillation experiments, the neutrino oscillation probabilities can be expressed as

$$
\begin{aligned}
P\left(\nu_{\mu} \rightarrow \nu_{e}\right) & \simeq \sin ^{2} 2 \theta_{13} \cdot \sin ^{2} \theta_{23} \cdot \sin ^{2} \Delta_{23} \\
& \equiv \sin ^{2} 2 \theta_{\mu e} \cdot \sin ^{2} \Delta_{23} \\
P\left(\nu_{\mu} \rightarrow \nu_{\mu}\right) & \simeq 1-\sin ^{2} 2 \theta_{23} \cdot \cos ^{4} \theta_{13} \cdot \sin ^{2} \Delta_{23} \\
& \equiv 1-\sin ^{2} 2 \theta_{\mu \tau} \cdot \sin ^{2} \Delta_{23}, \\
P\left(\nu_{e} \rightarrow \nu_{e}\right) & \simeq 1-\sin ^{2} 2 \theta_{13} \cdot \sin ^{2} \Delta_{23},
\end{aligned}
$$

where $\Delta_{23} \equiv \Delta m_{23}^{2} L / 4 E, \sin ^{2} 2 \theta_{\mu e} \equiv \sin ^{2} 2 \theta_{13} \cdot \sin ^{2} \theta_{23}$, and $\sin ^{2} 2 \theta_{\mu \tau} \equiv \sin ^{2} 2 \theta_{23} \cdot \cos ^{4} \theta_{13}$. These expressions can be regarded as the case of two flavor oscillations with their effective mixing angles of $\theta_{\mu e}$ and $\theta_{\mu \tau}$. 


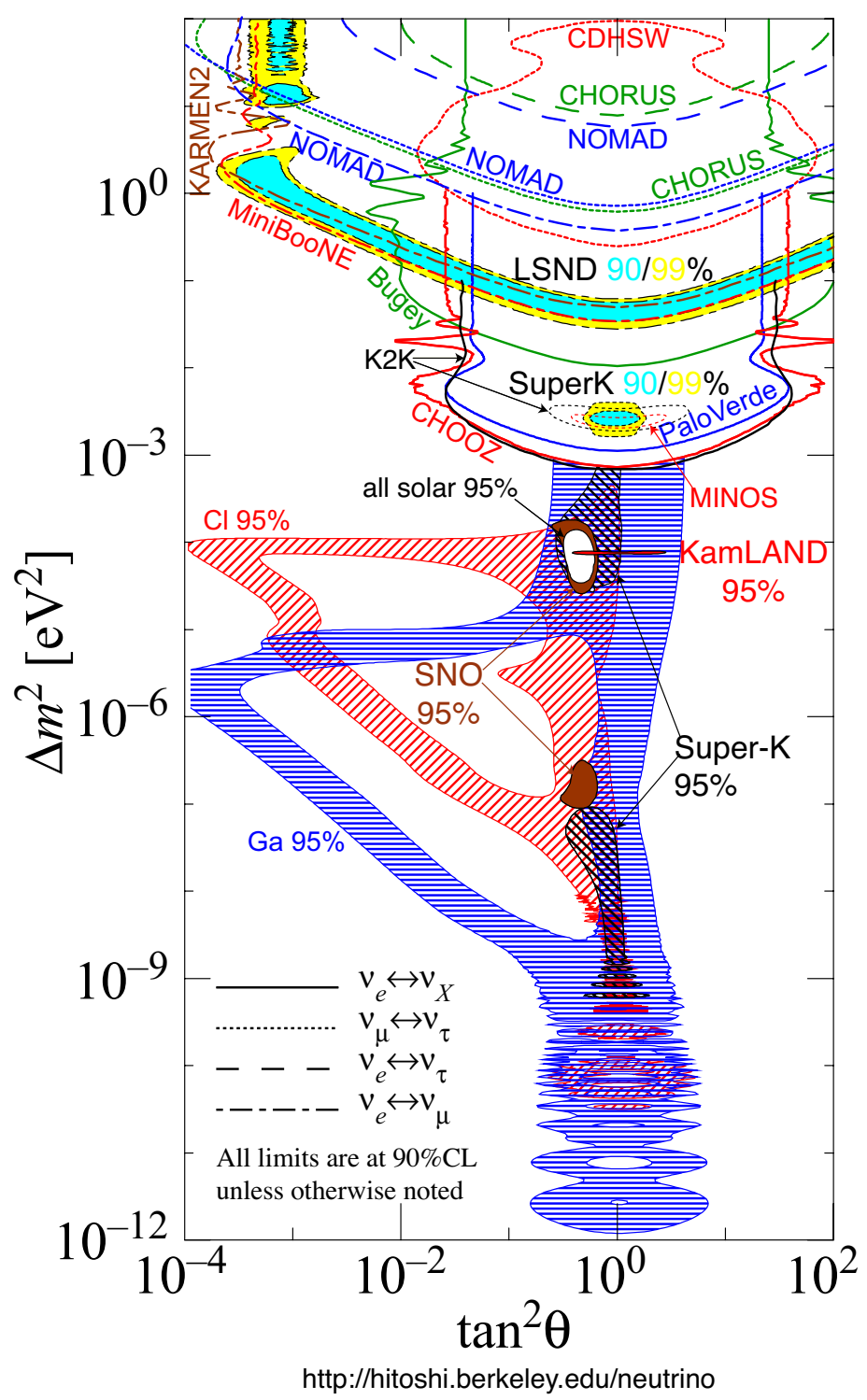

Figure 1.1: Allowed or excluded regions in the $\tan ^{2} \theta-\Delta m^{2}$ plane from various experiments.

\subsubsection{Summary of neutrino oscillation measurements}

Up to now, a number of experiments and observations to search for neutrino oscillation have been performed in the world. They are categorized into four types according to their source of neutrinos; atmospheric neutrino observations, solar neutrino observations, reactor neutrino experiments, and accelerator neutrino experiments. Figure 1.1 shows allowed or excluded regions from various experiments. In summary, there are two allowed regions:

1. Atmospheric region: $\Delta m^{2} \sim 2.5 \times 10^{-3} \mathrm{eV}^{2}, \theta \sim 45$ degrees

2. Solar region: $\Delta m^{2} \sim 8 \times 10^{-5} \mathrm{eV}^{2}, \theta \sim 30$ degrees 


\subsubsection{Future neutrino oscillation experiment}

The Tokai-to-Kamioka (T2K) experiment [2] is a next generation long-baseline neutrino oscillation experiment. The experiment will use a one-GeV muon neutrino beam produced by using a 50-GeV proton synchrotron at Japan Proton Accelerator Research Complex (J-PARC) in Japan. The properties of the neutrino beam are first measured by a near detector located at $280 \mathrm{~m}$ downstream from the proton target, and then neutrinos are detected by a 50 kton water Cherenkov detector, known as Super-Kamiokande, after traveling $295 \mathrm{~km}$. The goals of the T2K experiment are;

- Discovery of $\nu_{\mu} \rightarrow \nu_{e}$ oscillation

T2K searches for the last undiscovered oscillation channel, $\nu_{\mu} \rightarrow \nu_{e}$, which is related with the mixing angle $\theta_{13}$. The goal is to extend the search down to $\sin ^{2} 2 \theta_{13} \sim 0.008$.

- Precise measurement of oscillation parameters in $\nu_{\mu} \rightarrow \nu_{x}$ oscillation ( $\nu_{\mu}$ disappearance)

The experiment aims to measure the mixing angle $\theta_{23}$ and mass squared difference $\Delta m_{23}^{2}$ with an accuracy of $1 \%$ and $3 \%$, respectively.

- Search for sterile neutrinos $\left(\nu_{s}\right)$ in $\nu_{\mu}$ disappearance $\mathrm{T} 2 \mathrm{~K}$ can distinguish $\nu_{\mu} \rightarrow \nu_{\tau}$ oscillation from $\nu_{\mu} \rightarrow \nu_{s}$ oscillation at the region of $\Delta m^{2}>10^{-3} \mathrm{eV}^{2}$ by detecting neutral current events.

\section{$1.2 \quad$ Neutrino-nucleus interactions}

In the long-baseline neutrino oscillation experiments, neutrinos are detected via neutrinonucleus interactions because these interactions are predominant in the neutrino energy region of a few $\mathrm{GeV}$. In this section, we introduce the neutrino-nucleus interactions, the main topic of this thesis.

\subsubsection{Overview}

Neutrinos interact with nucleons in the nucleus (or entire nucleus) via both charged current $(\mathrm{CC})$ and neutral current $(\mathrm{NC})$ in the neutrino energy region of a few $\mathrm{GeV}$. The following processes are possible:

- quasi-elastic scattering $\left(\nu N \rightarrow \ell N^{\prime}\right)$

- single pion production $\left(\nu N \rightarrow \ell N^{\prime} \pi\right.$, or $\left.\nu A \rightarrow \ell A \pi\right)$

- deep inelastic scattering $\left(\nu N \rightarrow \ell N^{\prime}+\right.$ hadrons $)$

where $N$ and $N^{\prime}$ are the nucleons (proton or neutron), $A$ is the nucleus, $\ell$ is the lepton. Figure 1.2 shows the total muon neutrino charged current cross sections in the neutrino energy range between 0.1 and $100 \mathrm{GeV}$, overlaid with data from several experiments. In general, there are only a handful of cross section measurements in the few-GeV neutrino energy range, and their precision is limited by small statistics.

When we use nuclear targets, there are remarkable effects compared to neutrino interactions with a free nucleon. The Fermi motion of the initial state nucleon in the nucleus 


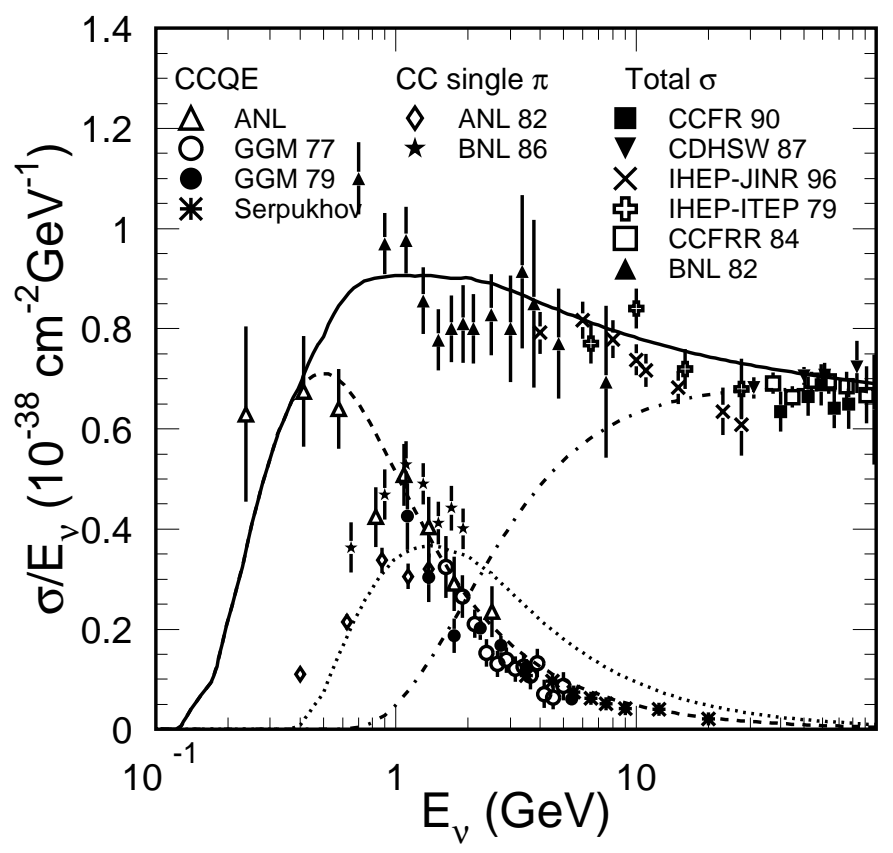

Figure 1.2: Charged current total cross section divided by neutrino energy for neutrino nucleon charged current interactions. The solid line shows the calculated total cross section. The dashed, dot and dash-dotted lines show the calculated quasi-elastic, single-meson and deep-inelastic scatterings, respectively. The data points are taken from the following experiments: $(\triangle) \operatorname{ANL}[3],(\bigcirc) \operatorname{GGM} 77[4],(\bullet) G G M 79(\mathrm{a})[5],(\mathrm{b})[6]$,

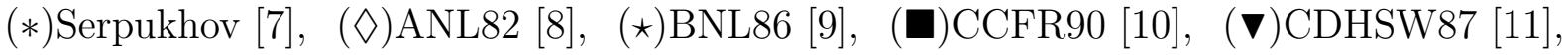

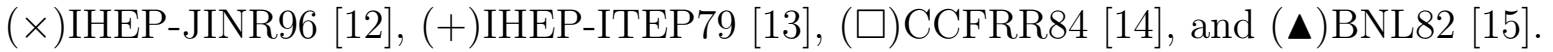

affects the kinematics of the final state particles. The Pauli exclusion principle suppresses the cross section at the small momentum transfer. The intra-nuclear interactions of mesons and nucleons produced in the nucleus change the momentum and direction of the particles. These effects are generally referred to as nuclear effects.

\subsubsection{Importance of neutrino-nucleus cross section measurements}

In this section, we discuss the importance of neutrino-nucleus cross section measurements for future neutrino oscillation experiments. Especially, we focus on the precise measurement of oscillation parameters in $\nu_{\mu}$ disappearance in the T2K experiment since this is the motivation of this thesis.

As discussed in Section 1.1.2, the survival probability of $\nu_{\mu}$ after traveling distance $L$ is expressed as

$$
P\left(\nu_{\mu} \rightarrow \nu_{\mu}\right)=1-\sin ^{2} 2 \theta_{\mu \tau} \cdot \sin ^{2} \frac{\Delta m_{23}^{2} L}{E_{\nu}},
$$

where $\theta_{\mu \tau}$ is the effective mixing angle, $\Delta m_{23}^{2}$ is the mass squared difference, and $E_{\nu}$ is the neutrino energy. With a fixed distance, the probability is a function of neutrino energy, and thus a distortion of the $\nu_{\mu}$ energy spectrum occurs. 

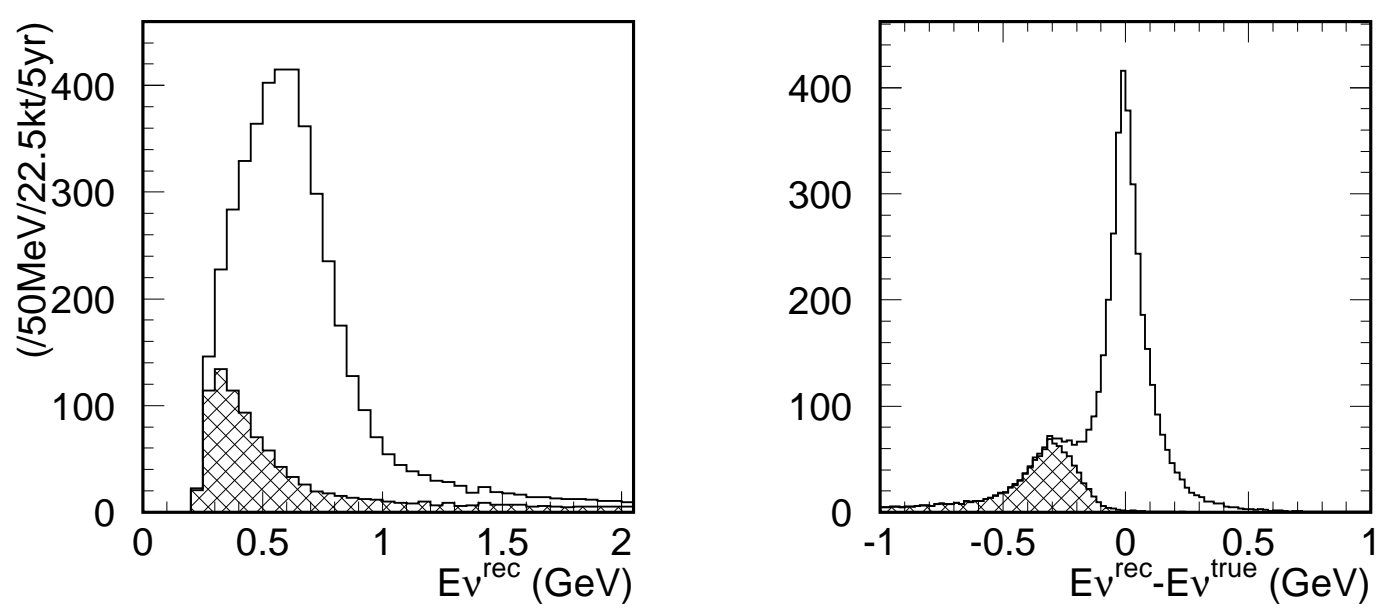

Figure 1.3: Expected distribution of the reconstructed neutrino energy assuming the CCQE kinematics (left), and the energy resolution in the case of no oscillation (right). The hatched histogram shows the background contribution.

In the long-baseline neutrino oscillation experiments, the energy spectrum distortion is measured by comparing the observations at the near site and at the far detector. In the case of T2K, the $\nu_{\mu}$ energy spectrum is measured with charged current quasi-elastic (CCQE) interactions, $\nu_{\mu} n \rightarrow \mu^{-} p$, which is the dominant process in this energy range. Since the CC-QE interaction is a two-body interaction, one can reconstruct neutrino energy from the measured muon momentum $\left(p_{\mu}\right)$ and angle with respect to the neutrino beam $\left(\theta_{\mu}\right)$, as

$$
E_{\nu}^{r e c}=\frac{1}{2} \frac{\left(m_{p}^{2}-m_{\mu}^{2}\right)-\left(m_{n}-V\right)^{2}+2 E_{\mu}\left(m_{n}-V\right)}{\left(m_{n}-V\right)-E_{\mu}+p_{\mu} \cos \theta_{\mu}},
$$

where $m_{p}, m_{n}$ and $m_{\mu}$ are the mass of proton, neutron and muon, respectively. $V$ is the nuclear potential. Figure 1.3 shows the expected distribution of the reconstructed neutrino energy at the far detector assuming the CC-QE kinematics in the case of no oscillation. The energy resolution is also shown. The hatched histogram shows the background contribution which is referred to as non-QE events. The diagrams of the signal and background process for the $\nu_{\mu}$ disappearance study are illustrated in Figure 1.4. The non-QE background is dominated by the charged current single charged pion production $\left(\mathrm{CC}-1 \pi^{+}\right)$in which the pion is not observed so that the final state looks like a CC-QE interaction. In T2K using the water Cherenkov detector, this mis-identification comes from the lack of the final state $\pi^{+}$detection due to low energy as well as pion absorption inside the nucleus.

Figure 1.5 shows the expected distribution of the reconstructed neutrino energy in the case of $\Delta m_{23}^{2}=2.7 \times 10^{-3} \mathrm{eV}^{2}$ and $\sin ^{2} 2 \theta_{23}=1$. The data points show the fake events generated with the MC simulation, and the hatched histogram shows the non$\mathrm{QE}$ background contribution. The non-QE background accounts for about half of the observed events when neutrino oscillation occurs.

Figure 1.6 shows the ratio of the reconstructed neutrino energy distribution with oscillation to the one without oscillation. The position and depth of the dip in the figure correspond to the size of $\Delta m_{23}^{2}$ and $\sin ^{2} 2 \theta_{23}$, respectively. If there exists a large uncertainty in the non-QE background estimation, the position and depth of the dip are 


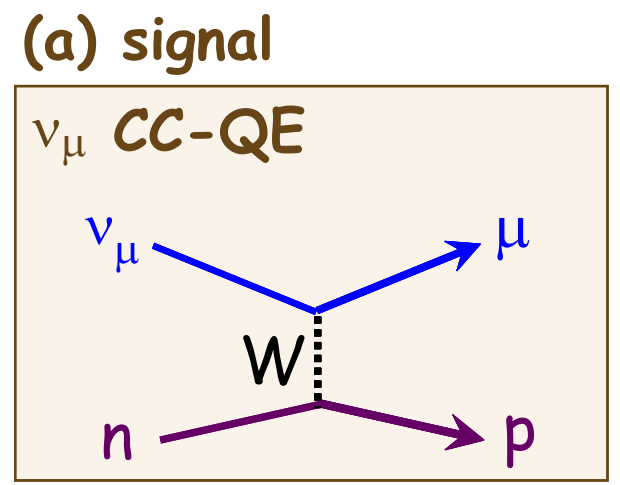

(b) background

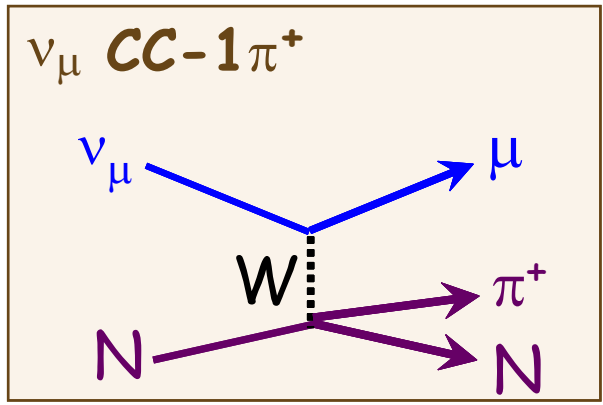

Figure 1.4: The diagrams of the signal and background processes for the $\nu_{\mu}$ disappearance study.

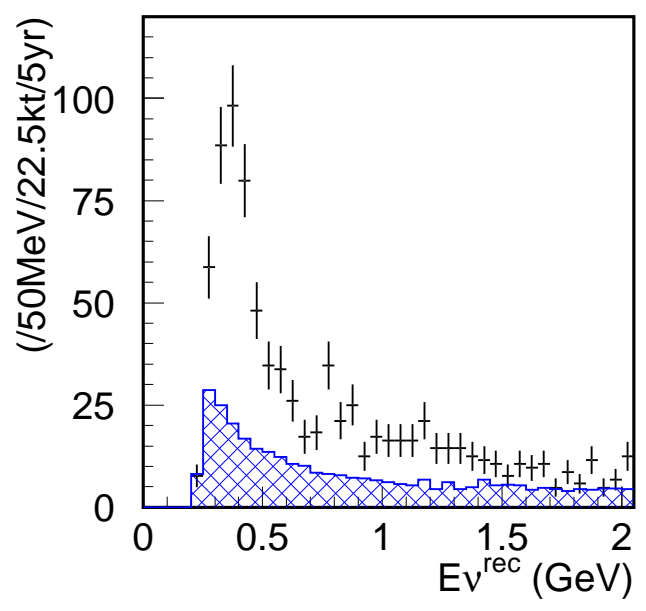

Figure 1.5: Expected distribution of the reconstructed neutrino energy in the case of $\Delta m_{23}^{2} \sim 2.7 \times 10^{-3} \mathrm{eV}^{2}$ and $\sin ^{2} 2 \theta_{23} \sim$ 1.

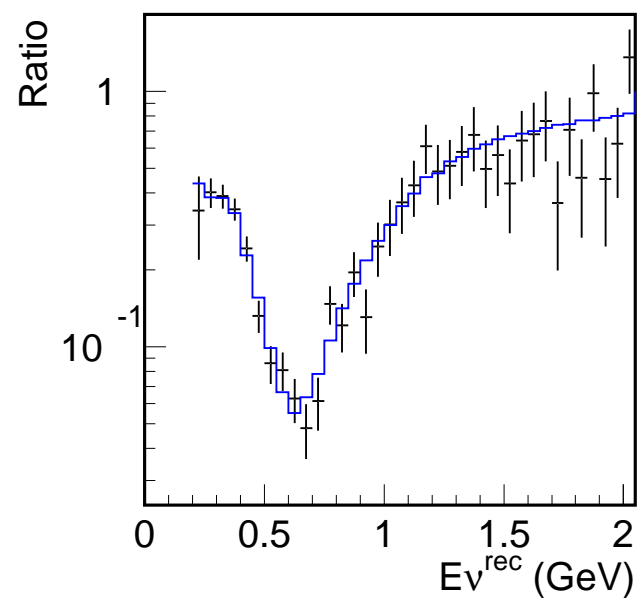

Figure 1.6: Ratio of the reconstructed neutrino energy distribution with oscillation to one without oscillation.

affected, and thus this can be a dominant source of the systematic uncertainty in the measurement of the oscillation parameters. The impact of the uncertainty in the nonQE background estimation on those oscillation parameters in T2K has been studied in Ref. [16]. Figure 1.7 shows the size of the measurement error in the oscillation parameters, $\sin ^{2} 2 \theta_{23}$ and $\Delta m_{23}^{2}$, as a function of true value of $\Delta m_{23}^{2}$ in various cases of the systematic uncertainty. The non-QE/CC-QE cross section ratio is desired to be understood at a $5 \%$ level to keep the resulting error on the oscillation parameters comparable to that due to statistical uncertainties. Since the non-QE background is dominated by charged current single charged pion production, the requirement is essentially equivalent to a $5 \%$ precision of the CC- $1 \pi^{+} / \mathrm{CC}-\mathrm{QE}$ cross section ratio. Whereas, the current knowledge of the cross section ratio is at a $20 \%$ level, and therefore further measurements of single pion production cross section are needed. 

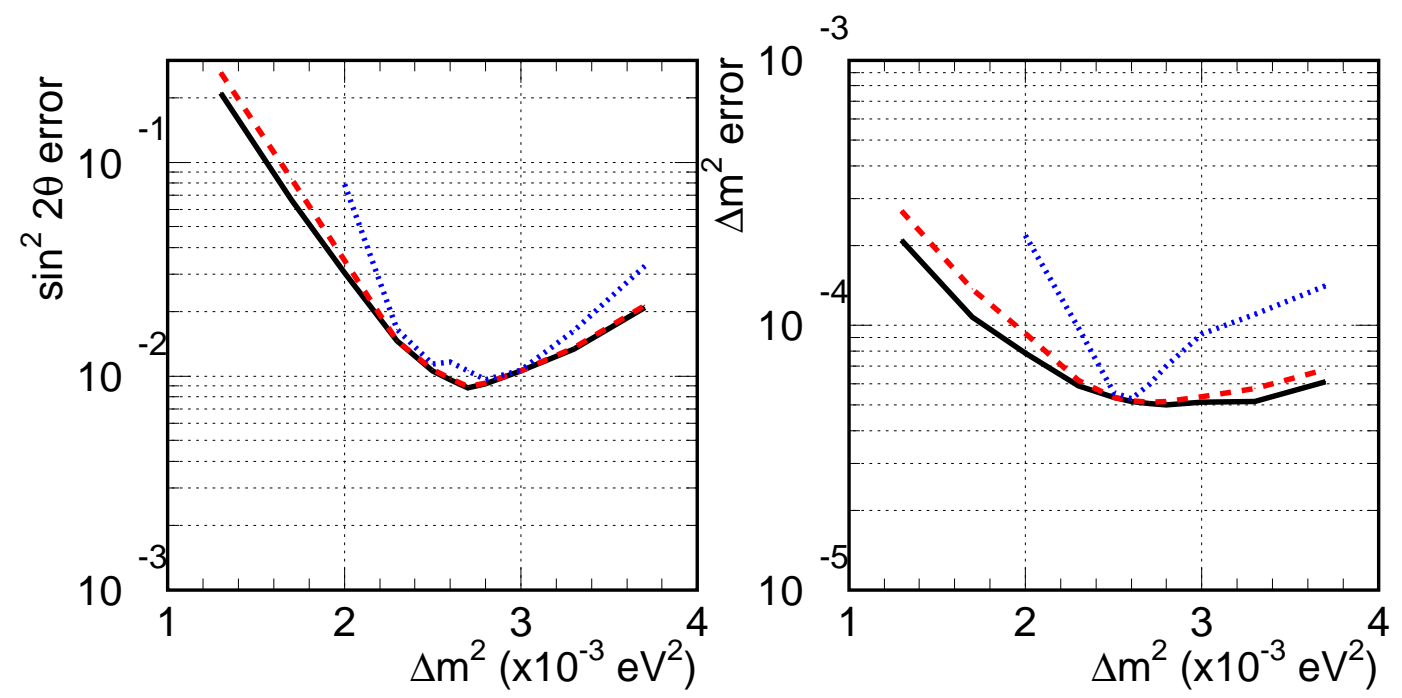

Figure 1.7: Size of the measurement error in the oscillation parameters, $\sin ^{2} 2 \theta_{23}$ and $\Delta m_{23}^{2}$, as a function of true value of $\Delta m_{23}^{2}$ in various cases of the systematic uncertainty. The solid line shows the case of statistical error only. The dashed and dotted lines shows the cases with a $5 \%$ and $20 \%$ uncertainty in the non-QE/CC-QE cross section ratio, respectively.

\subsubsection{Single pion production}

There are two processes which contribute to single pion production via neutrinonucleus interaction: resonant pion production and coherent pion production. In this section, we describe current knowledge of these processes, and emphasize the importance of precise measurements of these cross sections.

\section{Resonant pion production}

Single pion production is dominated by a baryonic resonance excitation off a single nucleon bound in a nucleus. The resonance state is followed by its prompt decay into a nucleon and a pion in the final state;

$$
\begin{array}{ll}
\nu N \rightarrow \ell \quad & N^{*} \\
& N^{*} \rightarrow N^{\prime} \pi,
\end{array}
$$

where $N$ and $N^{\prime}$ are nucleons (proton or neutron), or it is simply written as $\nu N \rightarrow$ $\ell N^{\prime} \pi$. Both charged current (CC) and neutral current (NC) resonant pion productions are possible. Considering charged pion production via charged current interaction by neutrinos, there are two channels:

$$
\begin{aligned}
& \nu_{\mu} p \rightarrow \mu^{-} p \pi^{+} \\
& \nu_{\mu} n \rightarrow \mu^{-} n \pi^{+} .
\end{aligned}
$$

A number of experimental measurements of resonant pion production via charged current interactions have been performed in the past 40 years, covering the neutrino energy range between 0.2 and $200 \mathrm{GeV}$, as shown in Table 1.1. Measurements of an early 
Table 1.1: Past measurements of charged current resonant pion production by neutrinos.

\begin{tabular}{lcccc}
\hline \hline Experiment & & $E_{\nu}(\mathrm{GeV})$ & Target & Final state \\
\hline Gargamelle & Lerche 1978 & $1-10$ & Propane & $\mu^{-} p \pi^{+}$ \\
BEBC & Allen 1980 & $5-200$ & $\mathrm{H}_{2}$ & $\mu^{-} p \pi^{+}$ \\
& Allen 1986 & $10-80$ & $\mathrm{H}_{2}$ & $\mu^{-} p \pi^{+}$ \\
& Allasia 1990 & $5-150$ & $\mathrm{D}_{2}$ & $\mu^{-} p \pi^{+}, \mu^{-} p \pi^{0}, \mu^{-} n \pi^{+}$ \\
BNL & Kitagaki 1986 & $0.5-14.5$ & $\mathrm{D}_{2}$ & $\mu^{-} p \pi^{+}, \mu^{-} p \pi^{0}, \mu^{-} n \pi^{+}$ \\
ANL & Campbell 1973 & $0.4-6.0$ & $\mathrm{H}_{2}, \mathrm{D}_{2}$ & $\mu^{-} p \pi^{+}$ \\
& Barish 1979 & $0.2-1.5$ & $\mathrm{H}_{2}, \mathrm{D}_{2}$ & $\mu^{-} p \pi^{+}, \mu^{-} n \pi^{+}$ \\
& Radecky 1982 & $0.3-1.5$ & $\mathrm{H}_{2}, \mathrm{D}_{2}$ & $\mu^{-} p \pi^{+}, \mu^{-} p \pi^{0}, \mu^{-} n \pi^{+}$ \\
FNAL & Bell 1978 & $10-100$ & $\mathrm{H}_{2}$ & $\mu^{-} p \pi^{+}$ \\
SKAT & Ammosov 1988 & $4-18.4$ & Heavy Freon & $\mu^{-} p \pi^{+}$ \\
& Grabosch 1989 & $3.5-12$ & Heavy Freon & $\mu^{-} p \pi^{+}, \mu^{-} p \pi^{0}, \mu^{-} n \pi^{+}$ \\
K2K & Rodriguez 2008 & $0.4-3$ & Polystyrene & $\mu^{-} N \pi^{+}$ \\
\hline \hline
\end{tabular}
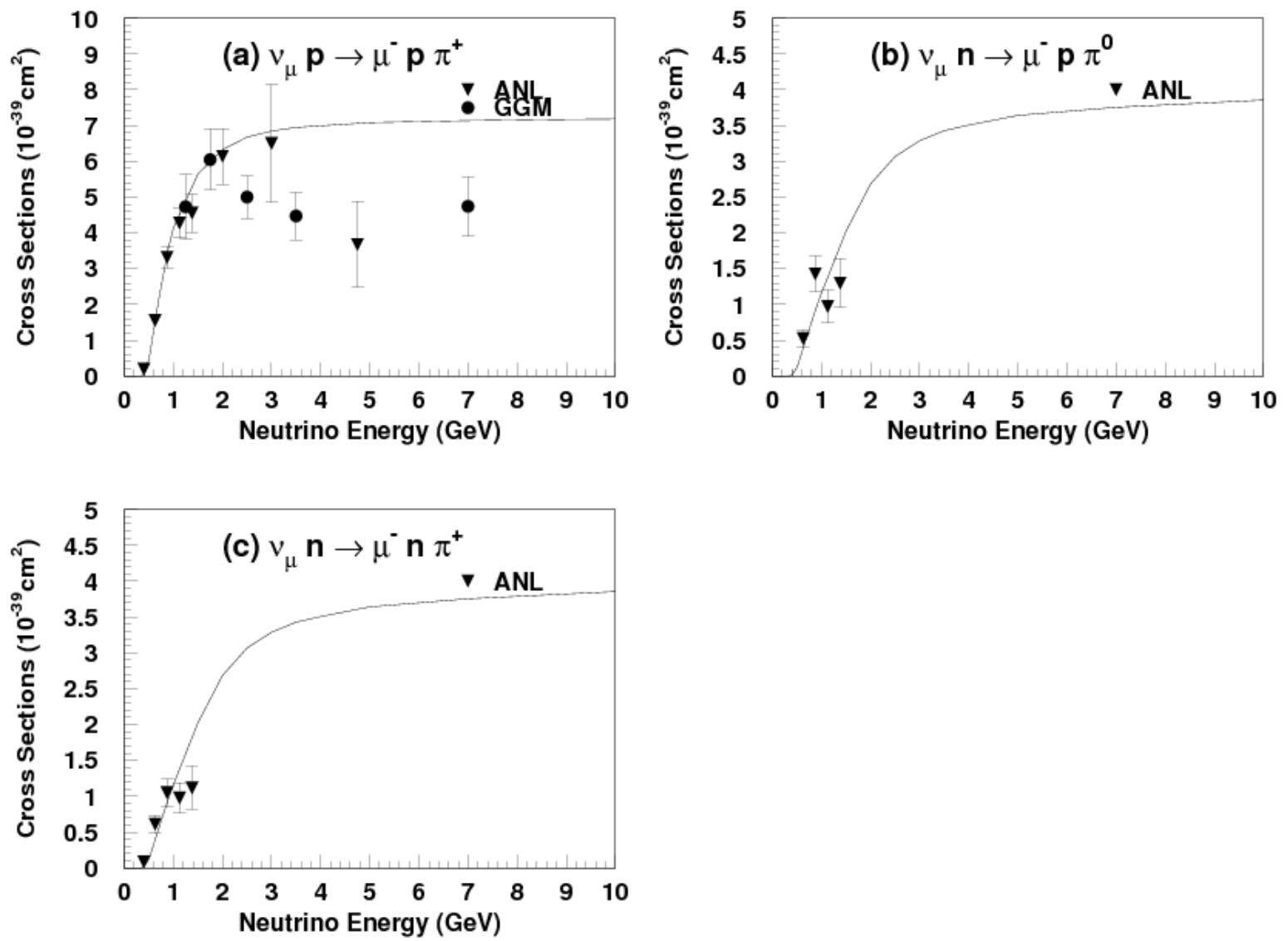

Figure 1.8: Experimental results on charged current resonant pion production cross sections in the neutrino energy region of a few $\mathrm{GeV}$. 
Table 1.2: Measured cross section ratios of charged current resonant charged pion production to charged current quasi-elastic scattering in the K2K experiment. The uncertainties in the measurement due to fitting errors, nuclear effects, and all other systematic effects are labeled "fit," "nucl," and "sys," respectively.

\begin{tabular}{ll}
\hline \hline Energy range $(\mathrm{GeV})$ & Cross section ratio $R_{k}=\sigma_{k}^{\text {res }} / \sigma_{k}^{\mathrm{QE}}$ \\
\hline$>0.00$ & $0.734 \pm 0.086$ (fit $)_{-0.103}^{+0.076}(\mathrm{nucl})_{-0.073}^{+0.079}(\mathrm{sys})$ \\
\hline $0.00-1.35$ & $0.402 \pm 0.111$ (fit $)_{-0.071}^{+0.079}(\mathrm{nucl})_{-0.092}^{+0.131}(\mathrm{sys})$ \\
$1.35-1.72$ & $1.022 \pm 0.167(\text { fit })_{-0.217}^{+0.072}(\mathrm{nucl})_{-0.139}^{+0.107}(\mathrm{sys})$ \\
$1.72-2.22$ & $1.007 \pm 0.214(\text { fit })_{-0.065}^{+0.209}(\mathrm{nucl})_{-0.173}^{+0.241}($ sys $)$ \\
$>2.22$ & $1.450 \pm 0.324(\text { fit })_{-0.200}^{+0.200}(\text { nucl })_{-0.480}^{+0.330}($ sys $)$ \\
\hline \hline
\end{tabular}

date were performed in bubble chamber experiments: ANL [8,17,18], Gargamelle [19,20], FNAL [21,22], BEBC [23-25], BNL [9], SKAT [26,27]. Hence, many results were obtained using light nuclei such as hydrogen and deuterium. Figure 1.8 shows experiment results on the charged current resonant pion production cross section in the neutrino energy range of a few $\mathrm{GeV}$. There are only a few experimental results in this energy region, and the precision of the measurements is at a $20 \%$ level, limited by low statistics.

The K2K Collaboration has recently published the result on the cross section ratio of charged current resonant pion production to charged current quasi-elastic scattering with a $1.3 \mathrm{GeV}$ wide band neutrino beam [28]. Table 1.2 shows the measured cross section ratios of charged current resonant charged pion production to charged current quasi-elastic scattering in the K2K experiment. The result, however, is limited by large systematic uncertainties mainly due to nuclear effects, and statistics are also not sufficient to measure the cross section with a precision better than $10 \%$. The knowledge of the cross section of this process has not reached to the precision required from future neutrino oscillation experiments, and therefore, further precision measurement of charged current resonant pion production cross section is essential.

\section{Coherent pion production}

In addition to resonant pion production, neutrinos can produce pions by interacting coherently with the nucleons forming the target nucleus. The general feature of the coherent process is that the four-momentum transfer to the target nucleus, $t$, is small so that the effective dimensions of space involved in the interactions is large compared with the dimensions of the target, i.e.,

$$
|t|<\frac{1}{R}
$$

where $R$ is the radius of the nucleus. There may not be any transfer of charge, spin, isospin, or any other quantum numbers. For example, if isospin were not conserved, the individual amplitudes for neutrons and protons would have opposite signs, destroying coherence. Because of the small momentum transfer to the target nucleus the outgoing lepton and pion tend to go in the forward direction in the lab frame, and no nuclear breakup occurs. Both charged current $(\mathrm{CC})$ and neutral current (NC) coherent pion 
Table 1.3: List of past measurements of coherent pion production.

\begin{tabular}{lcccccc}
\hline \hline Experiment & Beam & Reaction & $E_{\nu}(\mathrm{GeV})$ & Target & $\langle A\rangle$ & Reference \\
\hline Aachen-Padova & $\nu_{\mu} / \bar{\nu}_{\mu}$ & $\mathrm{NC}$ & 2 & $\mathrm{Al}$ & 27 & {$[38]$} \\
Gargamelle & $\nu_{\mu} / \bar{\nu}_{\mu}$ & $\mathrm{NC}$ & 3.5 & Freon & 30 & {$[39]$} \\
SKAT & $\nu_{\mu} / \bar{\nu}_{\mu}$ & $\mathrm{CC} / \mathrm{NC}$ & $3-30$ & Freon & 30 & {$[40]$} \\
CHARM & $\nu_{\mu} / \bar{\nu}_{\mu}$ & $\mathrm{NC}$ & $10-160$ & Marble & 20 & {$[41]$} \\
CHARM II & $\nu_{\mu} / \bar{\nu}_{\mu}$ & $\mathrm{CC}$ & $3-300$ & Glass & 20.1 & {$[42]$} \\
BEBC (WA59) & $\nu_{\mu} / \bar{\nu}_{\mu}$ & $\mathrm{CC}$ & $5-150$ & $\mathrm{Ne}$ & 20 & {$[43,44]$} \\
FNAL E632 & $\nu_{\mu} / \bar{\nu}_{\mu}$ & $\mathrm{CC}$ & $10-300$ & $\mathrm{Ne}$ & 20 & {$[45,46]$} \\
K2K & $\nu_{\mu}$ & $\mathrm{CC}$ & 1.3 & $\mathrm{C}$ & 12 & {$[47]$} \\
MiniBooNE & $\nu_{\mu}$ & $\mathrm{NC}$ & 1.2 & $\mathrm{C}$ & 12 & {$[48]$} \\
\hline \hline
\end{tabular}

productions are possible;

$$
\begin{aligned}
& \nu_{\mu} A \rightarrow \mu^{-} A \pi^{+} \\
& \nu_{\mu} A \rightarrow \nu_{\mu} A \pi^{0},
\end{aligned}
$$

where $A$ is a nucleus.

Several theoretical models describing coherent pion production have been proposed, using different formalisms to describe the relevant physics. A first class of models is built on the basis of Adler's Partially Conserved Axial-vector Current (PCAC) theorem [29], relating the neutrino-nucleus cross section to that of a pion interacting with a nucleus at $Q^{2}=0$, where $Q^{2} \equiv-\left(P_{\ell}-P_{\nu}\right)^{2}$ is the square of the four-momentum transfer, and $P_{\ell}$ and $P_{\nu}$ are the four-momenta of the outgoing lepton and the incoming neutrino, respectively; the extrapolation to $Q^{2} \neq 0$ is performed via a propagator term [30-34]. A second commonly-used formalism is based on the description of the coherent production of $\Delta$ resonances on nuclei by using a modified $\Delta$-propagator and a distorted wave-function for the pion [35-37].

The model of Rein and Sehgal [31,34], one of the first formalisms, is commonly used in neutrino oscillation experiments. Figure 1.9 shows the cross sections for $\nu_{\mu}{ }^{12} \mathrm{C} \rightarrow \mu^{-} \pi^{+12} \mathrm{C}$ interaction. The solid line represents the Rein and Sehgal model with lepton mass effects [34], the dashed line represents the Rein and Sehgal model without lepton mass effects [31]. The cross sections predicted by some of the recent models are also shown in the figure. While the relationship between neutral current and charged current modes, and that between neutrino and antineutrino coherent pion production cross sections, are relatively well known, order-of-magnitude variations on absolute coherent pion production cross sections are expected within these models. In addition, the cross section dependence on neutrino energy and on target material is also uncertain. It is therefore imperative that more experimental input on coherent pion production in neutrino-nucleus interactions is gathered in the near future.

Coherent pion production in neutrino-nucleus interactions has already been the subject of several experimental campaigns. The neutrino energy range between 1 and $100 \mathrm{GeV}$ has been investigated, including both the charged current and neutral current modes, and using both neutrino and antineutrino probes. Table 1.3 summarizes the past measurements of coherent pion production. The first evidence for neutral current coherent 


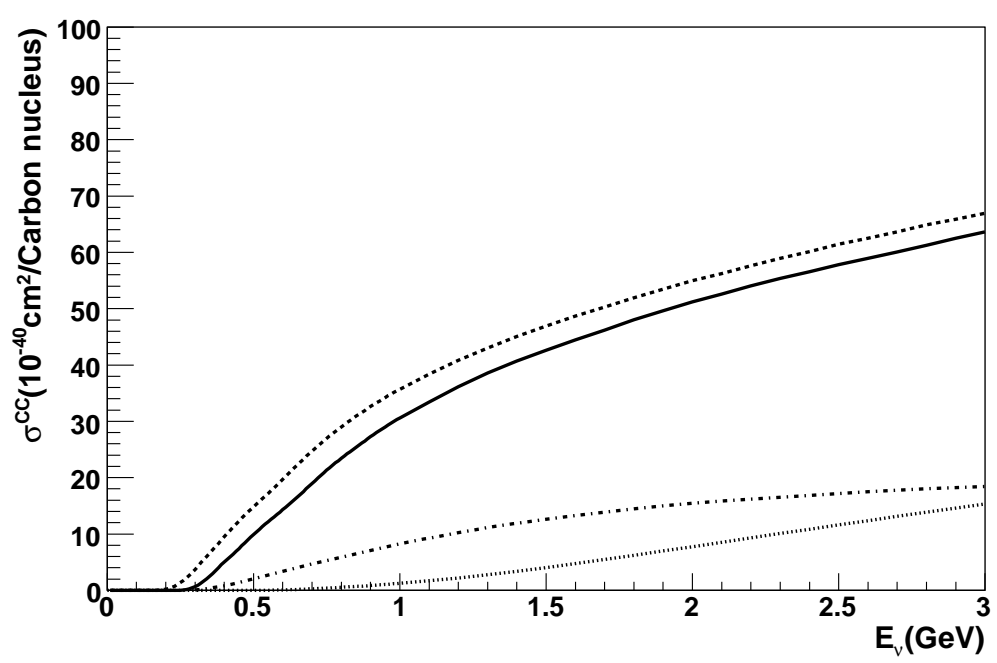

Figure 1.9: Cross section for $\nu_{\mu}{ }^{12} \mathrm{C} \rightarrow \mu^{-} \pi^{+12} \mathrm{C}$ interaction. The solid line represents the Rein and Sehgal model with lepton mass effects [34], the dashed line represents the Rein and Sehgal model without lepton mass effects [31], the dotted line represents the model of Kartavtsev et al. [33], and the dashed-dotted line represents the model of Alvarez-Ruso et al. [37]. The model of Singh et al. [35] gives a cross section similar to the model of Alvarez-Ruso et al.

pion production in a neutrino energy range of a few $\mathrm{GeV}$ has been reported from the Aachen-Padova [38] and Gargamelle [39] experimental data. Then, there exist coherent pion production positive results at higher energies (3-300 GeV neutrino energy) from the SKAT [40], CHARM [41], CHARM II [42], BEBC [43,44] and FNAL E632 [45,46] experiments.

A result that has drawn much attention in the neutrino physics community has been the recent non-observation of charged current coherent pion production by the $\mathrm{K} 2 \mathrm{~K}$ experiment with a $1.3 \mathrm{GeV}$ wide-band neutrino beam [47]. This is motivated by the fact that the K2K collaboration has quoted an upper limit for the ratio of the charged current coherent pion production cross-section to the charged current inclusive cross-section: $\sigma(\mathrm{CC}$ coherent $\pi) / \sigma(\mathrm{CC})<0.60 \times 10^{-2}$ at $90 \%$ confidence level. This is well below the prediction of the original Rein-Sehgal model [31] that has been adopted in the past to describe coherent pion production processes. In addition, even within more recently proposed models, it is often difficult to reconcile this new and accurate null result at low energies with previous measurements.

On the other hand, neutral current coherent pion production is observed by the MiniBooNE Collaboration [48] in a neutrino energy range that is similar to K2K. The fraction of neutral current coherent pion production to all neutral current single neutral pion production is found to be $(19.5 \pm 1.1$ (stat) \pm 2.5 (sys) $) \%$, while the fraction predicted based on the Rein-Sehgal model is $30 \%$. Although the measured fraction is significantly lower than the prediction, it is difficult to explain both the null result of charged current coherent pion production and the positive result on the neutral current channel in the similar neutrino energy with the same target nucleus at the same time.

Figure 1.10 shows a summary of existing experimental results on the coherent pion production cross section below $20 \mathrm{GeV}$. The results are scaled to the cross section for 


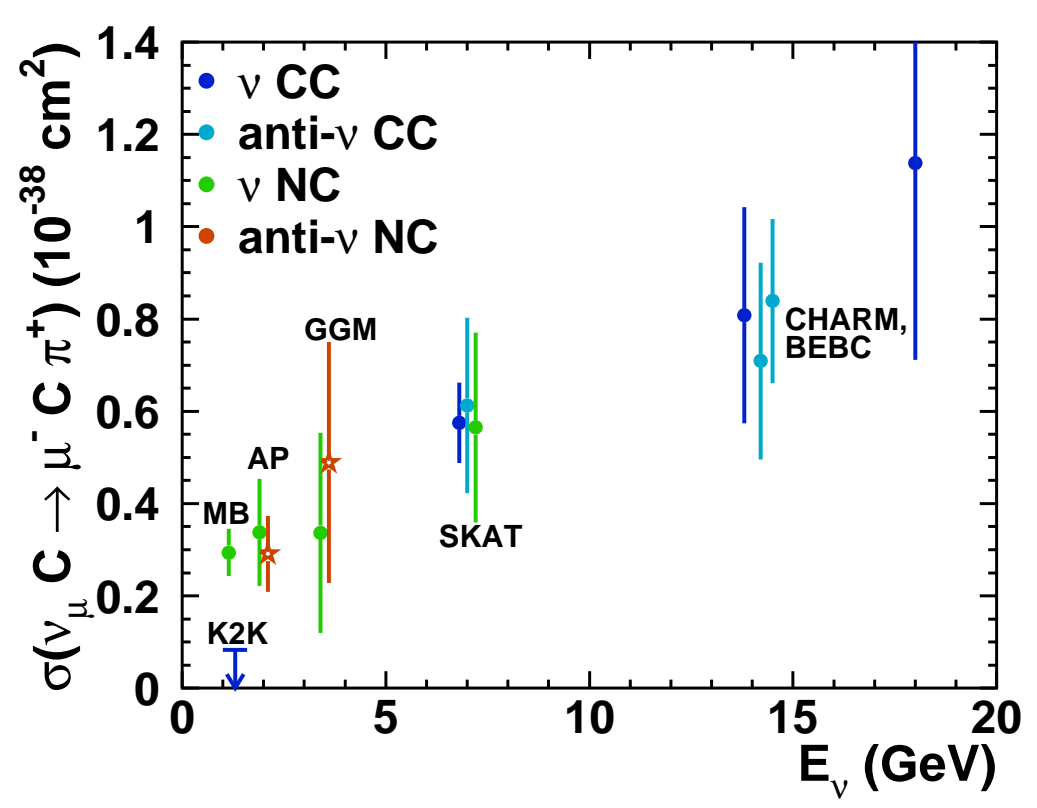

Figure 1.10: Existing experimental results on the coherent pion production cross section $\left(E_{\nu}<20 \mathrm{GeV}\right)$. The results are scaled to the cross section for $\nu_{\mu}{ }^{12} \mathrm{C} \rightarrow \mu^{-} \pi^{+12} \mathrm{C}$ by assuming (a) $\sigma(\mathrm{CC})=2 \sigma(\mathrm{NC})$, (b) $\sigma\left(\nu_{\mu}\right)=\sigma\left(\overline{\nu_{\mu}}\right)$, and (c) $A^{2 / 3}$ dependence. For the $\mathrm{K} 2 \mathrm{~K}$ result, the upper limit on the cross section ratio to total charged current is converted using the predicted value of the total charged current cross section quoted in their paper. Similarly, for the MiniBooNE result, the cross section ratio to all neutral current single neutral pion production is converted using the Rein-Sehgal prediction for resonant pion production.

$\nu_{\mu}{ }^{12} \mathrm{C} \rightarrow \mu^{-} \pi^{+12} \mathrm{C}$ under the following assumptions: (a) $\sigma(\mathrm{CC})=2 \sigma(\mathrm{NC})$, (b) $\sigma\left(\nu_{\mu}\right)=$ $\sigma\left(\overline{\nu_{\mu}}\right)$, and (c) $A^{2 / 3}$ dependence. The Rein-Sehgal model well explains these experimental measurements except for results from the K2K and MiniBooNE experiments.

In summary, there is a $100 \%$ uncertainty in the model prediction of charged current coherent pion production cross section in the neutrino energy regime of a few $\mathrm{GeV}$. In addition, recent experimental results in the charged current and neutral current channels are contradictory. Whereas, the model prediction of charged current resonant pion production is relatively reliable, and the uncertainty in the cross section measurement is at a $20 \%$ level. Although resonant pion production is dominant in this neutrino energy range, coherent pion production comprises $\sim 10 \%$ of single charged pion production, and the contribution is especially large in the region of small muon scattering angle. Therefore, the precise determination of resonant pion production cross section can not be achieved without measuring the coherent component first.

\subsection{Overview of this thesis}

The purpose of the study presented in this thesis is a precise measurement of charged current single charged pion productions, resonant and coherent pion productions, with a good final state separation in the neutrino energy region of a few $\mathrm{GeV}$. In this thesis, 
we focus on the measurement of charged current coherent pion production using the full neutrino data from the SciBooNE experiment. This is motivated by the fact that the precise determination of resonant pion production cross section can not be achieved without measuring the coherent component first since the contribution of coherent pion production in the region of small muon scattering angle is large. Furthermore, the coherent process is particularly interesting because it is deeply rooted in fundamental physics via Adler's PCAC theorem.

This thesis is organized as follows. Chapter 2 describes the overview of the SciBooNE experiment. In Chapter 3, we describe the neutrino beamline and the neutrino flux simulation. The simulation of neutrino interactions with nuclei is described in Chapter 4. The detector configuration and simulation are described in Chapter 5. A summary of the data set and experimental performance is given in Chapter 6. In Chapter 7, we discuss a study of charged current coherent pion production. The charged current event selection, tuning of the Monte Carlo (MC) simulation, the extraction of coherent pion events, and the measurement of the ratio of charged current coherent pion production to total charged current cross sections are described in detail. The discussion of future prospects of charged current single charged pion production measurements is presented in Chapter 8, and conclusions are given in Chapter 9. 


\section{Chapter 2}

\section{SciBooNE Experiment}

In this chapter, we describe the overview, history, and physics motivations of the SciBooNE experiment.

\subsection{Overview of SciBooNE}

The SciBar Booster Neutrino Experiment (SciBooNE) [49] is designed for measuring the neutrino-nucleus cross sections around one $\mathrm{GeV}$ region, which is essential for future neutrino oscillation experiments such as T2K. We briefly summarize features of the SciBooNE experiment;

- High intensity low energy neutrino beam

SciBooNE uses the Booster Neutrino Beam (BNB) at Fermi National Accelerator Laboratory (FNAL) which has been used for the MiniBooNE experiment. The BNB can provide a high rate, low energy neutrino beam. The beamline can also provide an antineutrino beam by reversing the polarity of the horn current.

- Fully active fine segmented scintillator tracking detector

The experiment uses Scintillator Bar (SciBar) detector, a fully active fine segmented tracking detector. The SciBar detector was originally developed for the $\mathrm{K} 2 \mathrm{~K}$ experiment [50]. SciBar acts as the neutrino target, and also can detect all charged particles produced by neutrino interactions.

The marriage of a high intensity low energy neutrino beam and the fine granularity of SciBar is a unique and efficient opportunity for precise measurements of neutrino cross sections since both are already built and have been operated successfully.

Figure 2.1 shows a schematic drawing of the experimental setup of SciBooNE. The SciBooNE detector is positioned $100 \mathrm{~m}$ downstream from the proton target on the axis of the beam. The MiniBooNE detector is located $440 \mathrm{~m}$ downstream from the SciBooNE detector, exposed to the same neutrino beam. The detector comprises three sub-detectors; the SciBar detector is followed by an electromagnetic calorimeter (EC) and a muon range detector (MRD). Detailed descriptions of the BNB and SciBooNE detector are given in Chapter 3 and Chapter 5, respectively. 


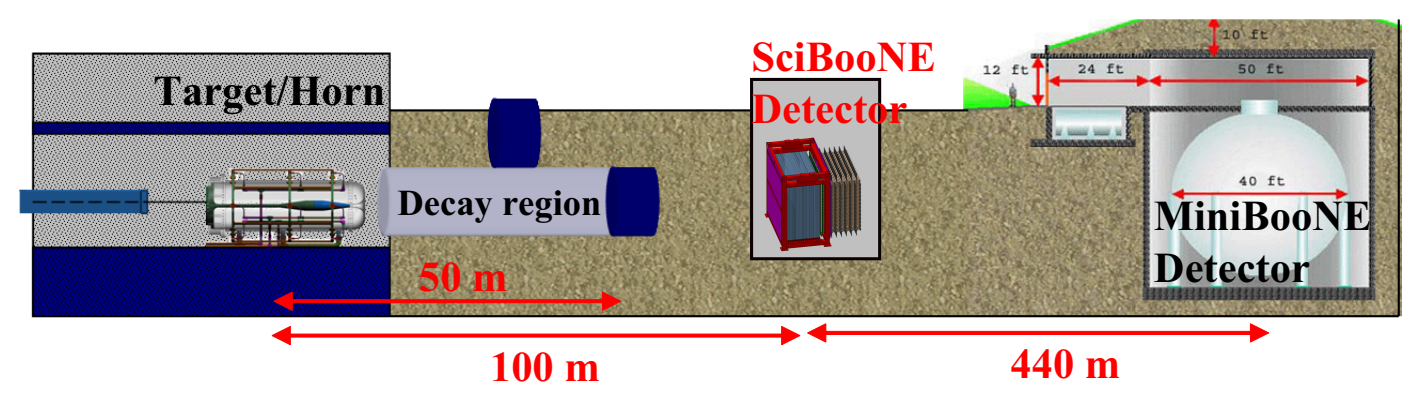

Figure 2.1: Schematic drawing of the experimental setup of SciBooNE.

\subsection{History of SciBooNE}

We summarize a history of the SciBooNE experiment in Table 2.1. The idea of the experiment came up in early 2005 which is right after the completion of the K2K experiment. The SciBooNE Collaboration was formed in summer 2005, physics case studies were performed [51,52], and we submitted a proposal [49] to FNAL Physics Advisory Committee (PAC). The proposal was approved by FNAL PAC in December 2005. The SciBar and EC detectors in the K2K experiment were disassembled at KEK between November 2005 and February 2006. We moved the SciBar and EC components from KEK to FNAL in July 2006. The civil construction of the SciBooNE detector hall started in September 2006. From November 2006 to March 2007, the SciBar/EC and MRD detectors were built up and tested with cosmic-ray muons separately, in parallel with the detector hall construction. In April 2007, all the sub-detectors were installed into the detector hall, and then we started integrating sub-detector systems and commissioning. We started data-taking first with antineutrino beam in June 2007 since the MiniBooNE experiment was already running in the antineutrino mode. We continued data-taking until the summer accelerator shutdown in August 2007 [53]. The period is called Run-I. After the accelerator shutdown, we took data with neutrino beam by reversing the horn

Table 2.1: History of the SciBooNE experiment.

\begin{tabular}{ll}
\hline \hline 2005 Summer & Collaboration was formed. \\
2005 Dec. & Proposal was approved by FNAL PAC (FNAL E954). \\
2005 Nov. - 2006 Feb. & The SciBar/EC detectors were disassembled at KEK. \\
2006 Jul. & The SciBar/EC components were moved to FNAL. \\
2006 Sep. & Civil construction of the detector hall was started. \\
2006 Nov. - 2007 Mar. & Sub-detectors were built up and tested with cosmic-rays. \\
2007 Apr. & Detector was installed into the detector hall. \\
2007 May. & Sub-detector systems were merged and commissioned. \\
2007 Jun. - 2007 Aug. & Data-taking with antineutrino beam (Run-I). \\
2007 Oct. - 2008 Apr. & Data-taking with neutrino beam (Run-II). \\
2008 Apr. - 2008 Aug. & Data-taking with antineutrino beam (Run-III). \\
2008 Aug. & SciBooNE completed data-taking. \\
\hline \hline
\end{tabular}




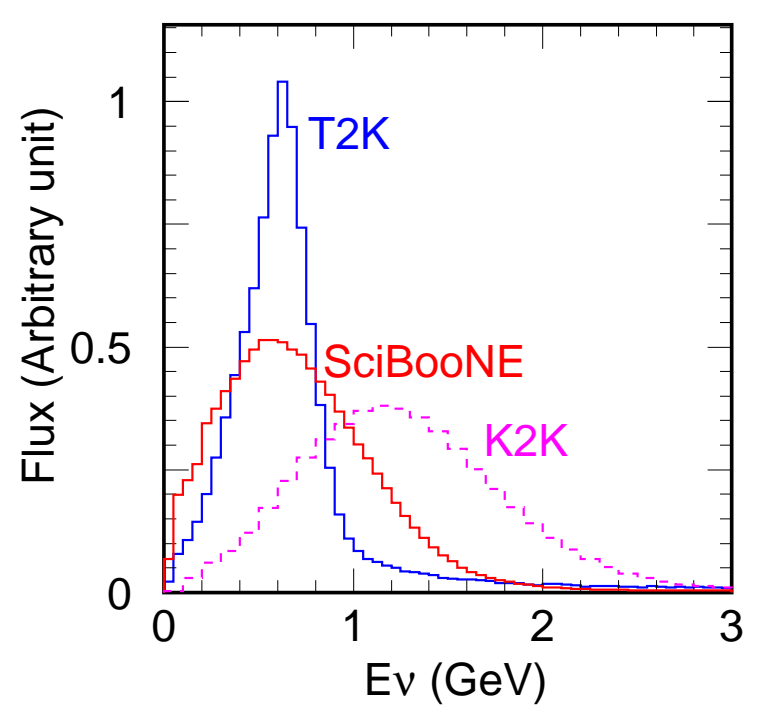

Figure 2.2: Comparison of the muon neutrino energy spectra at $\mathrm{K} 2 \mathrm{~K}, \mathrm{~T} 2 \mathrm{~K}$, and SciBooNE. All curves are normalized to unit area.

polarity from October 2007 until April 2008 (Run-II). Then, we switched back to the antineutrino mode data-taking, and kept running until August 2008 (Run-III). Since we have successfully collected sufficient amount of data as requested, in both neutrino and antineutrino beams, we completed data-taking and the detector was de-commissioned in August 2008.

\subsection{Physics motivations of SciBooNE}

The physics motivations of SciBooNE can be put into three categories: precise measurements of neutrino-nucleus cross sections, measurements of antineutrino-nucleus cross sections, and neutrino flux measurements.

\subsubsection{Precise measurements of neutrino-nucleus cross sections}

Figure 2.2 shows the $\nu_{\mu}$ energy spectrum at SciBooNE as well as those at K2K and $\mathrm{T} 2 \mathrm{~K}$. The entire range of the T2K energy spectrum is encompassed within the spectrum of SciBooNE. Given similarity of neutrino spectra as shown in the figure, various neutrino cross section measurements at SciBooNE will help neutrino oscillation studies in T2K. The study presented in this thesis is classified into this category.

\subsubsection{Measurements of antineutrino-nucleus cross sections}

If there are indications of a finite $\theta_{13}$, T2K will step forward to search for CP violation in the neutrino sector. It requires oscillation measurements with both neutrino and antineutrino beam. However, the current knowledge of antineutrino cross sections in the few $\mathrm{GeV}$ range is very poor, even less understood compared with neutrino cross sections, with only a handful of low statistics measurements to date. SciBooNE can provide various antineutrino cross section measurements as well. 


\subsubsection{Neutrino flux measurements}

The SciBooNE detector can also provide a measured constraint on the $\nu_{\mu}$ flux normalization and energy spectrum that can be used for neutrino oscillation searches at MiniBooNE. The effects of flux normalization and shape systematics on the sensitivity to $\nu_{\mu} \rightarrow \nu_{x}$ and $\bar{\nu}_{\mu} \rightarrow \bar{\nu}_{x}$ oscillations have been studied [49], and indicate the utility of an external measurement of the neutrino flux. In the $\bar{\nu}_{\mu} \rightarrow \bar{\nu}_{x}$ oscillation search, it is crucial to understand the wrong-sign $\left(\nu_{\mu}\right)$ backgrounds, and the SciBooNE detector allows us to extract the normalization and energy spectrum of the backgrounds. 


\section{Chapter 3}

\section{Booster Neutrino Beam}

In this chapter, we describe apparatus to produce neutrino beam, and the neutrino flux prediction at the SciBooNE detector location.

\subsection{Beamline description}

\subsubsection{Primary proton beam}

The primary beam uses protons accelerated to $8 \mathrm{GeV}$ kinetic energy by the Fermilab Booster. Selected batches containing approximately $4-5 \times 10^{12}$ protons are extracted and bent toward the BNB target hall via dipole magnets. Each spill is composed of 81 bunches of protons, approximately $6 \mathrm{nsec}$ wide each and $19 \mathrm{nsec}$ apart, for a total spill duration of $1.6 \mu \mathrm{sec}$. One cycle is about $2 \mathrm{sec}$ which is defined by the accelerator timing sequence. The BNB receives one train of proton beam pulses per cycle, with a maximum of 10 pulses in a row at $15 \mathrm{~Hz}$.

Beam proton trajectories and positions are monitored on a pulse-by-pulse basis. The typical beam alignment and divergence, measured by the beam position monitors located near the target, are within $1 \mathrm{~mm}$ and $1 \mathrm{mrad}$ of the nominal target center and axis direction, respectively; the typical beam focusing on target measured by beam profile monitors is of the order of 1-2 mm (RMS) in both the horizontal and vertical directions. These parameters are well within the experiment requirements. The number of protons delivered to the BNB target is measured for each proton batch using two toroidal current transformers (often referred to as toroids) located near the target along the beamline. The gain and pedestal corrections for each toroid are performed using test current pulses on a pulse-by-pulse basis, and the absolute calibration is performed occasionally. These toroid calibrations provide a measurement of the number of protons to BNB with a $2 \%$ accuracy.

\subsubsection{Target and magnetic focusing horn}

The primary proton beam strikes a thick beryllium target located in the BNB target hall. Hadronic interactions of the protons with the target material produce a beam of secondary mesons (pions and kaons). The target is made of seven cylindrical slugs with a radius of $0.51 \mathrm{~cm}$, for a total target length of $71.1 \mathrm{~cm}$, or about 1.7 inelastic interaction lengths. 
The beryllium target is surrounded by a magnetic focusing horn, bending and signselecting the secondary particles that emerge from the interactions in the target along the direction pointing to the SciBooNE detector. The focusing is produced by the toroidal magnetic field present in the air volume between the horn's two coaxial conductors made of aluminum alloy. The horn current pulse is approximately a half-sinusoid of amplitude $174 \mathrm{kA}, 143 \mu \mathrm{sec}$ long, synchronized to each beam spill. Given the pulsed nature of the horn current, a residual magnetic field also penetrates the horn inner conductor (skin depth effect). The polarity of the horn current flow can be (and has been) switched, in order to focus negatively-charged mesons, and therefore produce an antineutrino instead of a neutrino beam.

\subsubsection{Decay region and absorber}

The secondary mesons emerging from the target/horn region are further collimated via passive shielding, and allowed to decay into neutrinos in a cylindrical decay region filled with air at atmospheric pressure, $50 \mathrm{~m}$ long and $90 \mathrm{~cm}$ in radius. A beam absorber located at the end of the decay region stops hadronic particles and muons, and only a pure neutrino beam pointing toward the detector remains, mostly from $\pi^{+} \rightarrow \mu^{+} \nu_{\mu}$ decays.

\subsection{Neutrino flux prediction}

In order to obtain neutrino flux predictions at the SciBooNE detector location, a Monte Carlo (MC) simulation, developed by the MiniBooNE Collaboration [54], is used. The simulation uses the GEANT4 framework [55].

\subsubsection{Simulation of meson productions}

In the simulation code, a realistic description of the geometry and materials present in the BNB target hall and decay region is implemented. Primary protons are generated according to the expected beam optics properties upstream of the target. The interactions of primary protons with the beryllium target are simulated according to state-of-the-art hadron interaction data. Production of secondary protons, neutrons, charged pions, and charged and neutral kaons is taken into account, and elastic and quasi-elastic scattering of protons in the target are also simulated. Of particular importance for these analyses is $\pi^{+}$production in proton-beryllium interactions, which uses experimental input from the HARP [56] and BNL E910 [57] experiments.

For $\pi^{+}, \pi^{-}$, and $K^{0}$ productions, the Sanford-Wang parametrization [58] is employed to calculate the double differential cross section of a given meson species:

$$
\frac{d^{2} \sigma}{d p d \Omega}=C_{1} p^{C_{2}}\left(1-\frac{p}{p_{B}-C_{9}}\right) \exp \left(-C_{3} \frac{p^{C_{4}}}{p_{B}^{C_{5}}}-C_{6} \theta\left(p-C_{7} p_{B} \cos ^{C_{8}} \theta\right)\right)
$$

where $p$ is the total momentum of the meson, $\theta$ is the angle of the meson with respect to the incident proton, $p_{B}$ is the momentum of the incident proton, and $C_{1}, \ldots, C_{9}$ are parameters determined by fitting to the experimental data. In the case of the $K^{0}$ production, since the particles are produced in strong interactions, the neutral kaons have equal 
Table 3.1: Sanford-Wang parameters used in the simulation

\begin{tabular}{cccccccccc}
\hline \hline & $C_{1}$ & $C_{2}$ & $C_{3}$ & $C_{4}$ & $C_{5}$ & $C_{6}$ & $C_{7}$ & $C_{8}$ & $C_{9}$ \\
\hline$\pi^{+}$ & 220.7 & 1.080 & 1.000 & 1.978 & 1.32 & 5.572 & 0.0868 & 9.686 & 1 \\
$\pi^{-}$ & 213.7 & 0.9379 & 5.454 & 1.210 & 1.284 & 4.781 & 0.07338 & 8.329 & 1 \\
$K_{S}^{0}$ & 15.130 & 1.975 & 4.084 & 0.928 & 0.731 & 4.362 & 0.048 & 13.300 & 1.278 \\
\hline \hline
\end{tabular}

Table 3.2: Feynman scaling parameters used in the simulation

\begin{tabular}{cccccccc}
\hline \hline & $C_{1}$ & $C_{2}$ & $C_{3}$ & $C_{4}$ & $C_{5}$ & $C_{6}$ & $C_{7}$ \\
\hline$K^{+}$ & 11.70 & 0.88 & 4.77 & 1.51 & 2.21 & 2.17 & 1.51 \\
\hline \hline
\end{tabular}

content as $K_{S}^{0}$ and $K_{L}^{0}$. As a result, the production properties of neutral kaon decaying as $K_{L}^{0}$ is obtained from the measured $K_{S}^{0}$ production data. The Sanford-Wang parameters used in the simulation are summarized in Table 3.1.

For $K^{+}$production, since no measurement exist at the BNB primary proton beam energy, the Feynman scaling hypothesis is employed to relate $K^{+}$production measurements at different proton beam energies to the expected production at the BNB proton beam energy. According to the hypothesis, the invariant cross section is a function of only two variables, namely the transverse component of the momentum of the produced particle, $p_{T}$, and the Feynman scaling variable, $x_{F} \equiv p_{\|}^{\mathrm{cm}} / p_{\|}^{\max , \mathrm{cm}}$, defined as the ratio of the parallel component of the momentum of the produced particle in the center-of-mass frame and the maximum value of this quantity for the given reaction. The double differential cross section using the Feynman scaling is expressed as:

$$
\frac{d^{2} \sigma}{d p d \Omega}=\frac{p^{2}}{E^{2}} C_{1}\left(1-\left|x_{F}\right|\right) \exp \left(-C_{2} p_{T}-C_{3}\left|x_{F}\right|^{C_{4}}-C_{5} p_{T}^{2}-C_{7}\left|p_{T} \times x_{F}\right|^{C_{6}}\right)
$$

where $p$ and $E$ are the momentum and energy of the produced meson, respectively. The Feynman scaling parameters used in the simulation are summarized in Table 3.2.

For $K^{-}$production, because of the scarcity of production measurements in the relevant kinematic regions, the MARS hadronic interaction package [59] is used to determine the absolute double differential cross section.

Particles emanating from the primary proton-beryllium interaction in the target are then propagated within the GEANT4 framework, which accounts for all relevant physics processes. Hadronic re-interactions of pions and nucleons with beryllium and aluminum materials are particularly important and are described by custom models, while other hadronic processes and all electromagnetic processes (energy loss, multiple scattering, effect of horn magnetic field, etc.) are described according to default GEANT4 physics lists. 
Table 3.3: Neutrino-producing decay modes considered in the simulation. The corresponding decays of negatively-charged particles are also simulated.

\begin{tabular}{lrlr}
\hline \hline Particle & $\begin{array}{r}\text { Lifetime } \\
(\mathrm{nsec})\end{array}$ & Decay mode & $\begin{array}{r}\text { Branching ratio } \\
(\%)\end{array}$ \\
\hline$\pi^{+}$ & 26.03 & $\mu^{+} \nu_{\mu}$ & 99.9877 \\
& & $e^{+} \nu_{e}$ & 0.0123 \\
\hline$K^{+}$ & 12.385 & $\mu^{+} \nu_{\mu}$ & 63.44 \\
& & $\pi^{0} e^{+} \nu_{e}$ & 4.98 \\
& & $\pi^{0} \mu^{+} \nu_{\mu}$ & 3.32 \\
\hline$K_{L}^{0}$ & 51.6 & $\pi^{-} e^{+} \nu_{e}$ & 20.333 \\
& & $\pi^{+} e^{-} \bar{\nu}_{e}$ & 20.197 \\
& & $\pi^{-} \mu^{+} \nu_{\mu}$ & 13.551 \\
& & $\pi^{+} \mu^{-} \bar{\nu}_{\mu}$ & 13.469 \\
\hline$\mu^{+}$ & 2197.03 & $e^{+} \nu_{e} \bar{\nu}_{\mu}$ & 100.0 \\
\hline \hline
\end{tabular}

\subsubsection{Simulation of meson decays}

A second, FORTRAN-based Monte Carlo code uses the output of the GEANT4 program as input, and is responsible for generating the neutrino kinematics distributions from meson and muon decays, and for obtaining the final neutrino fluxes extrapolated to the SciBooNE detector. Current best knowledge of meson and muon decay branching fractions, and decay form factors in three-body semi-leptonic decays, are used as summarized in Table 3.3. Polarization effects in muon decays are also accounted for.

\subsubsection{Neutrino flux prediction at SciBooNE}

Once produced by the simulation, neutrinos are extrapolated along straight lines toward the SciBooNE detector. All neutrinos whose ray traces cross any part of the detector volume are considered for SciBooNE flux predictions. Each simulated neutrino interaction is linked to its detailed beam information and history, which includes neutrino flavor, energy, parent type, and kinematics, and ray trace entry and exit points within the detector volume; the ray trace information is used to determine the incoming neutrino's direction and interaction location. Proper weights for each beam neutrino event are computed, using this beam neutrino information, as well as information from the interaction and detector simulation: neutrino interaction probability, and detailed SciBooNE detector geometry and specifications. No information from the SciBooNE and MiniBooNE neutrino flux measurements is used as experimental input for the neutrino beam simulation.

Figure 3.1 shows the neutrino flux predictions at the SciBooNE detector location and as a function of neutrino energy. The spectra are averaged within $2.12 \mathrm{~m}$ from the beam center. $^{1}$ In neutrino mode running (positive horn polarity), a total neutrino flux per proton on target of $2.2 \times 10^{-8} \mathrm{~cm}^{-2}$ is expected at the SciBooNE detector location, with a mean neutrino energy of $0.7 \mathrm{GeV}$. The flux is dominated by muon neutrinos $(93 \%$ of

\footnotetext{
${ }^{1}$ The area used in this calculation covers the cross-sectional area of the SciBar detector which is $3 \mathrm{~m} \times 3 \mathrm{~m}$.
} 

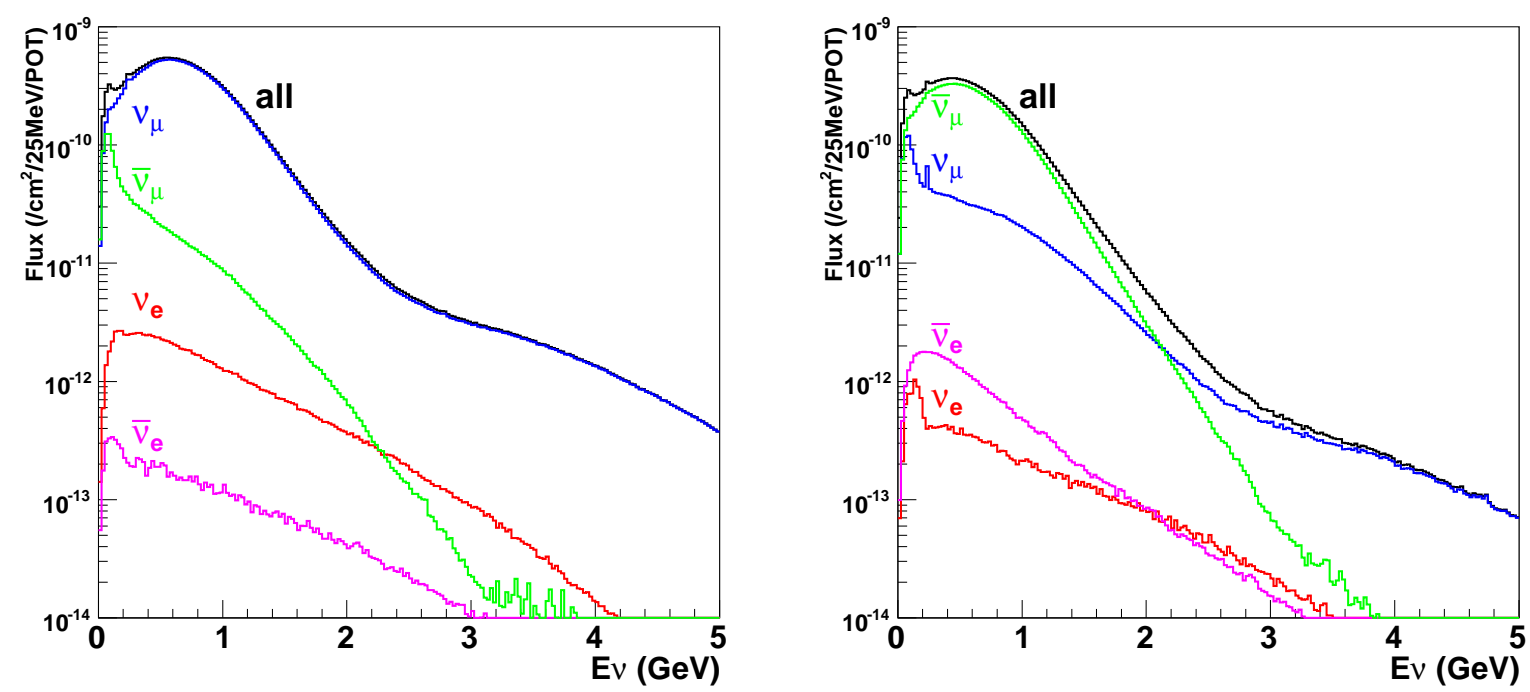

Figure 3.1: Neutrino flux predictions at the SciBooNE detector as a function of neutrino energy $E_{\nu}$, normalized per unit area, proton on target (POT) and neutrino energy bin width, in neutrino mode (left) and antineutrino mode running. The spectra are averaged within $2.12 \mathrm{~m}$ from the beam center. The total flux and contributions from individual neutrino flavors are shown.

total), with small contributions from muon antineutrinos (6.4\%), and electron neutrinos and antineutrinos ( $0.6 \%$ in total).

On the other hand, in antineutrino mode running (negative horn polarity), the expected total neutrino flux per proton on target is $1.3 \times 10^{-8} \mathrm{~cm}^{-2}$, and the mean neutrino energy is $0.6 \mathrm{GeV}$. The flux is dominated by muon antineutrinos ( $84 \%$ of total), with contributions from muon neutrinos (15.6\%), and electron neutrinos and antineutrinos $(0.7 \%$ in total).

\subsubsection{Systematic uncertainties in the neutrino flux prediction}

The systematic uncertainties in the neutrino flux prediction come from several sources;

- Proton delivery/optics: The predicted number of neutrino interactions in the detector varies directly with the uncertainty in the number of protons on target. The uncertainties in the overall flux normalization due to the proton beam intensity measurements and the proton beam optics are $2 \%$ and $1 \%$, respectively.

- Secondary particle productions: The uncertainties in the secondary particle production cross sections in proton-beryllium interactions affect the rate and spectrum of the neutrinos they produce. To simulate secondary particle productions, we use the Sanford-Wang model for $\pi^{+}, \pi^{-}$, and $K^{0}$ productions, and the Feynman scaling model is used for $K^{+}$production, as described in Section 3.2.1. The uncertainties in the fitted parameters are considered. This is the dominant uncertainty.

- Hadronic interactions in the target or horn: The uncertainties in the rate of these interactions affect both the rate and shape of the flux. To evaluate the 

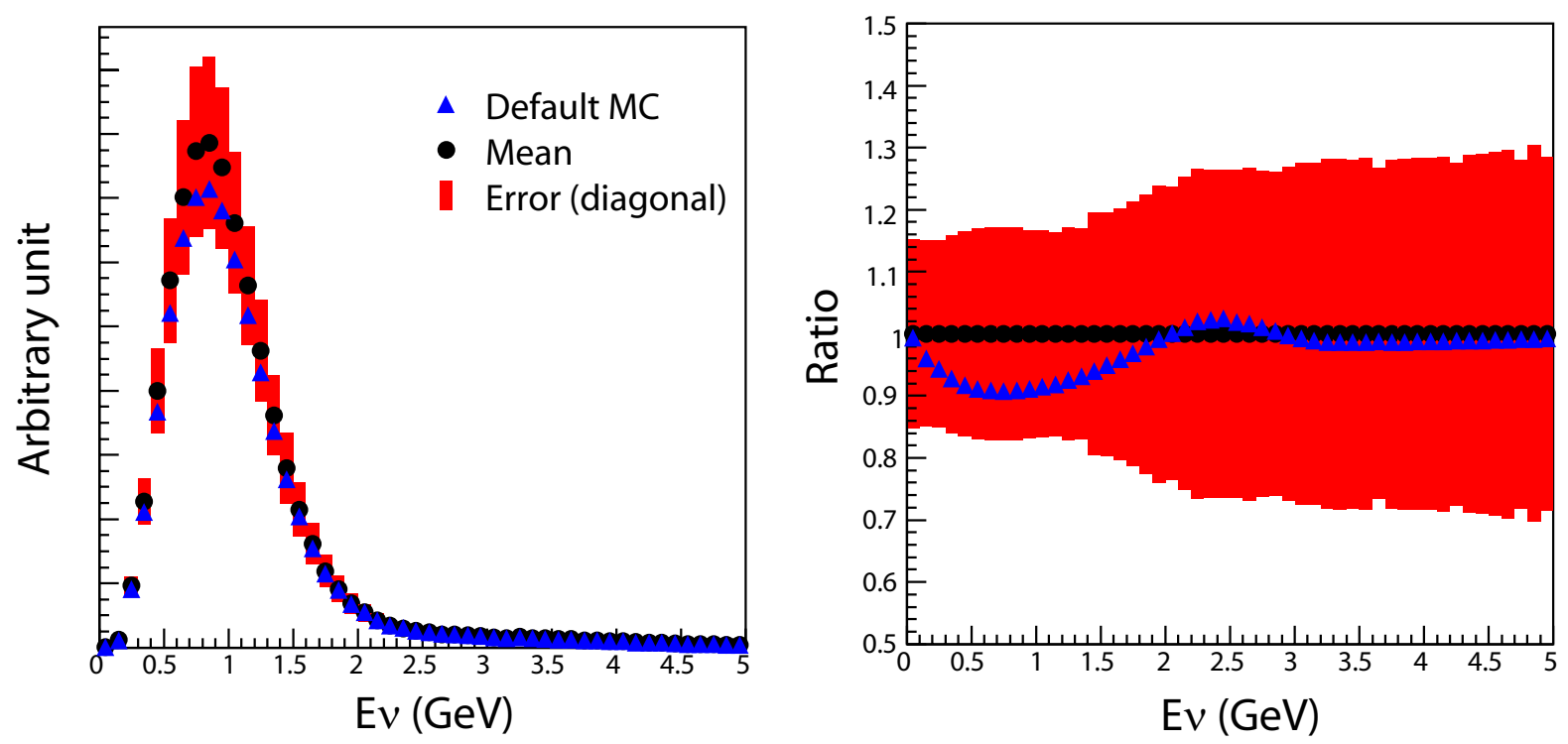

Figure 3.2: Systematic uncertainty in the neutrino energy distribution for the total interactions by muon neutrinos. The fractional uncertainty as a function of neutrino energy is also shown.

systematic uncertainties, the total hadronic cross section, the inelastic and quasielastic cross sections are separately varied for nucleons on beryllium and aluminum. The same is done for the pion cross sections.

- Horn magnetic field : The uncertainties in the horn magnetic field model result in spectral distortions of the neutrino flux. The horn current uncertainty of $\pm 1 \mathrm{kA}$ and the uncertainty in the modeling of the current within the inner cylinder due to the so-called "skin effect" are taken into account.

Detailed descriptions of each uncertainty are found elsewhere [54].

Figure 3.2 shows the neutrino energy distribution for the total interactions by muon neutrinos at the SciBooNE detector. The filled band indicates the uncertainty due to the beam systematics. The overall normalization error due to the proton delivery and optics is not included in the figure. The triangle points indicate the distribution obtained from the default MC simulation used in these analyses. Due to the complex parameterization of the Sanford-Wang and Feynman scaling formulae, the error band is asymmetric about the default MC simulation. The systematic uncertainty in the total number of interactions at the SciBooNE detector is estimated to be $15 \%$. The error is dominated by the uncertainty in the $\pi^{+}$production cross section, which is $14 \%$.

Because of the large uncertainty in the neutrino flux prediction, we measure cross section ratios in this thesis in order to reduce the systematic uncertainty, instead of measuring absolute cross sections. 


\section{Chapter 4}

\section{Neutrino Interaction Simulation (NEUT)}

The neutrino interactions with nuclear targets are simulated with the NEUT program library [60,61] which is used in the Kamiokande, Super-Kamiokande, K2K, and T2K experiments. NEUT handles protons, oxygen, carbon, and iron as nuclear targets in the energy range from $100 \mathrm{MeV}$ to $100 \mathrm{TeV}$. Following the primary neutrino interactions in nuclei, re-interactions of the mesons and hadrons with the nuclear medium are also simulated.

\subsection{Neutrino-nucleus interactions}

In NEUT, the following neutrino interactions in both charged current (CC) and neutral current $(\mathrm{NC})$ are simulated:

- quasi-elastic scattering $\left(\nu N \rightarrow \ell N^{\prime}\right)$

- single meson production $\left(\nu N \rightarrow \ell N^{\prime} m\right)$

- single gamma production $\left(\nu N \rightarrow \ell N^{\prime} \gamma\right)$

- coherent $\pi$ production $\left(\nu^{12} \mathrm{C}\left(\mathrm{or}^{56} \mathrm{Fe}\right) \rightarrow \ell \pi{ }^{12} \mathrm{C}\left(\mathrm{or}^{56} \mathrm{Fe}\right)\right)$

- deep inelastic scattering $\left(\nu N \rightarrow \ell N^{\prime} h a d r o n s\right)$

where $N$ and $N^{\prime}$ are the nucleons (proton or neutron), $\ell$ is the lepton, and $m$ is the meson. Figure 4.1 shows neutrino-nucleus cross sections per nucleon divided by neutrino energy. The expected number of $\nu_{\mu}$ interactions in the SciBar fiducial volume at SciBooNE is summarized in Table 4.1.

\subsubsection{Quasi-elastic scattering}

The dominant interaction in the SciBooNE neutrino energy range is quasi-elastic scattering, which is implemented using the model of Llewellyn-Smith [62]. The amplitude of 


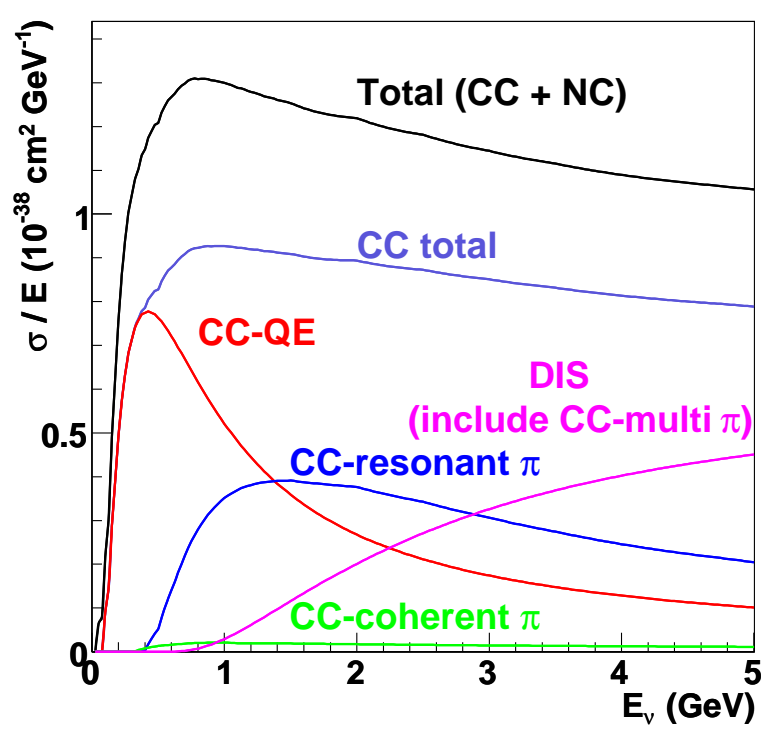

Figure 4.1: Neutrino-nucleus cross sections per nucleon divided by neutrino energy.

Table 4.1: Expected number of $\nu_{\mu}$ interactions in the SciBar fiducial volume at SciBooNE.

\begin{tabular}{lr}
\hline \hline Mode & $\begin{array}{r}\text { Number of } \nu_{\mu} \text { interactions } \\
\left(/ 10 \text { tons } / 10^{20} \text { POT }\right)\end{array}$ \\
\hline CC QE & 50,800 \\
CC resonant $\pi$ & 26,200 \\
CC coherent $\pi$ & 1,700 \\
CC DIS & 6,300 \\
NC elastic & 21,400 \\
NC resonant $\pi$ & 10,400 \\
NC coherent $\pi$ & 1,000 \\
NC DIS & 2,000 \\
\hline \hline
\end{tabular}

this process is described by the product of the leptonic and hadronic weak currents. The hadronic current is expressed as

$$
<N^{\prime}\left|J_{\mu}^{\mathrm{had}}\right| N>=\cos \theta_{c} \bar{u}\left(N^{\prime}\right)\left[\gamma_{\mu} F_{V}^{1}\left(Q^{2}\right)+\frac{i \sigma_{\mu \nu} q^{\nu} \xi F_{V}^{2}\left(Q^{2}\right)}{2 m_{N}}+\gamma_{\mu} \gamma_{5} F_{A}\left(Q^{2}\right)\right] u(N),
$$

where $\theta_{c}$ is the Cabbibo angle, $\xi \equiv \mu_{p}-\mu_{n}=3.71$ is the difference of anomalous dipole moments between a proton and a neutron, and $m_{N}$ is the nucleon mass. The vector form factors, $F_{V}^{1}$ and $F_{V}^{2}$, are represented as

$$
\begin{aligned}
F_{V}^{1}\left(Q^{2}\right) & =\left(1+\frac{Q^{2}}{4 m_{N}^{2}}\right)^{-1}\left[G_{E}^{V}\left(Q^{2}\right)+\frac{Q^{2}}{4 m_{N}^{2}} G_{M}^{V}\left(Q^{2}\right)\right], \\
\xi F_{V}^{2}\left(Q^{2}\right) & =\left(1+\frac{Q^{2}}{4 m_{N}^{2}}\right)^{-1}\left[G_{M}^{V}\left(Q^{2}\right)-G_{E}^{V}\left(Q^{2}\right)\right]
\end{aligned}
$$


where $G_{E}^{V}$ and $G_{M}^{V}$ are the electric and magnetic Sachs form factors, given by

$$
G_{E}^{V}\left(Q^{2}\right)=\frac{1}{\left(1+\frac{Q^{2}}{M_{V}^{2}}\right)^{2}} \quad, \quad G_{M}^{V}\left(Q^{2}\right)=\frac{1+\xi}{\left(1+\frac{Q^{2}}{M_{V}^{2}}\right)^{2}} .
$$

The axial-vector form factor, $F_{A}$, is given by

$$
F_{A}\left(Q^{2}\right)=\frac{g_{A}}{\left(1+\frac{Q^{2}}{M_{A}^{2}}\right)^{2}}
$$

where $g_{A}=-1.23$ is determined from neutron decay measurements.Both the vector and axial-vector form factors are assumed to be dipole. The vector mass, $M_{V}$, is set to be $0.84 \mathrm{GeV} / c^{2}$. The axial vector mass, $M_{A}$, is set to be $1.21 \mathrm{GeV} / c^{2}$ as suggested by recent results $[63,64]$.

The differential cross section is expressed as

$$
\frac{d \sigma}{d Q^{2}}=\frac{m_{N}^{2} G_{F}^{2} \cos ^{2} \theta_{c}}{8 \pi E_{\nu}^{2}}\left[A\left(Q^{2}\right) \mp B\left(Q^{2}\right) \frac{s-u}{m_{N}^{2}}+C\left(Q^{2}\right) \frac{(s-u)^{2}}{m_{N}^{4}}\right],
$$

where $E_{\nu}$ is the incident neutrino energy, $s-u \equiv 4 m_{N} E_{\nu}-Q^{2}-m_{\ell}^{2}, m_{\ell}$ is the mass of the outgoing lepton, and

$$
\begin{aligned}
A\left(Q^{2}\right)= & \frac{m_{\ell}^{2}+Q^{2}}{4 m_{N}^{2}}\left[\left(4+\frac{Q^{2}}{m_{N}^{2}}\right)\left|F_{A}\right|^{2}-\left(4-\frac{Q^{2}}{m_{N}^{2}}\right)\left|F_{V}^{1}\right|^{2}\right. \\
& +\frac{Q^{2}}{m_{N}^{2}}\left|\xi F_{V}^{2}\right|^{2}\left(1-\frac{Q^{2}}{4 m_{N}^{2}}\right)+\frac{4 Q^{2} F_{V}^{1} \xi F_{V}^{2}}{m_{N}^{2}} \\
& \left.-\frac{m_{\ell}^{2}}{m_{N}^{2}}\left(\left|F_{V}^{1}+\xi F_{V}^{2}\right|^{2}+\left|F_{A}\right|^{2}\right)\right], \\
B\left(Q^{2}\right)= & -\frac{Q^{2}}{m_{N}^{2}} F_{A}\left(F_{V}^{1}+\xi F_{V}^{2}\right), \\
C\left(Q^{2}\right)= & \frac{1}{4}\left(\left|F_{A}\right|^{2}+\left|F_{V}^{1}\right|^{2}+\frac{Q^{2}}{m_{N}^{2}}\left|\frac{\xi F_{V}^{2}}{2}\right|^{2}\right) .
\end{aligned}
$$

To obtain the cross sections for neutral current elastic scattering, we use the following relations $[65,66]$ :

$$
\begin{aligned}
\sigma(\nu p \rightarrow \nu p) & =0.153 \times \sigma\left(\nu n \rightarrow \mu^{-} p\right) \\
\sigma(\bar{\nu} p \rightarrow \bar{\nu} p) & =0.218 \times \sigma\left(\bar{\nu} p \rightarrow \mu^{+} n\right) \\
\sigma(\nu n \rightarrow \nu n) & =1.5 \times \sigma(\nu p \rightarrow \nu p) \\
\sigma(\bar{\nu} n \rightarrow \bar{\nu} n) & =1.0 \times \sigma(\bar{\nu} n \rightarrow \bar{\nu} n)
\end{aligned}
$$

For scattering off nucleons in the nucleus, we use the relativistic Fermi gas model of Smith and Moniz [67]. The nucleons are treated as quasi-free particles and the Fermi motion of nucleons along with the Pauli exclusion principle is taken into account. The momentum distribution of the target nucleon is assumed to be flat up to a fixed Fermi surface momentum of $217 \mathrm{MeV} / c$ for carbon and $250 \mathrm{MeV} / c$ for iron. The same Fermi momentum distribution is also used for all of the other nuclear interactions. The nuclear potential is set to $27 \mathrm{MeV}$ for carbon and $32 \mathrm{MeV}$ for iron. 


\subsubsection{Single meson production via baryon resonances}

The second most probable interaction in SciBooNE is the resonant single meson production of $\pi, K$, and $\eta$, described by the model of Rein and Sehgal [68]. The model assumes an intermediate baryon resonance, $N^{*}$ :

$$
\begin{aligned}
\nu N & \rightarrow \ell N^{*}, \\
N^{*} & \rightarrow N^{\prime} m \quad(m=\pi, \eta, K) .
\end{aligned}
$$

The double differential cross section of single meson production depends on the amplitude for the production of a given resonance and the probability of the baryon resonance decay to the meson:

$$
\frac{d^{2} \sigma}{d Q^{2} d \nu}=\frac{1}{32 \pi m_{N} E_{\nu}^{2}} \cdot \frac{1}{2} \sum_{\text {spins }}\left|T\left(\nu N \rightarrow \ell N^{*}\right)\right|^{2} \cdot \delta\left(W^{2}-M^{2}\right),
$$

where $W$ is the hadronic invariant mass, and $T\left(\nu N \rightarrow \ell N^{*}\right)$ is the amplitude of a given resonance production which is calculated using FKR (Feynman-Kislinger-Ravndal) baryon model [69]. The model includes the vector and axial-vector form factors using the dipole parametrization with the same $M_{V}$ and $M_{A}$ values as quasi-elastic scattering. For resonances with a finite decay width, the double differential cross sections can be derived by replacing the $\delta$-function with a Breit-Wigner formula:

$$
\delta\left(W^{2}-M^{2}\right) \rightarrow \frac{1}{2 \pi} \frac{\Gamma}{(W-M)^{2}+\Gamma^{2} / 4} .
$$

All intermediate baryon resonances with mass less than $2 \mathrm{GeV} / c^{2}$ are included. Those baryon resonances with mass greater than $2 \mathrm{GeV} / c^{2}$ are simulated as deep inelastic scattering. Lepton mass effects from the non-conservation of lepton current and the pion-pole term in the hadronic axial vector current are included in the simulation [70,71].

To determine the angular distribution of a pion in the final state, Rein's method [72] is used for the $P_{33}(1232)$ resonance. For other resonances, the directional distribution of the generated pion is set to be isotropic in the resonance rest frame. The angular distribution of $\pi^{+}$has been measured for $\nu_{\mu} p \rightarrow \mu^{-} p \pi^{+}[9]$ and the results agree well with NEUT's prediction. Pauli blocking is accounted for in the decay of the baryon resonance by requiring the momentum of the nucleon to be larger than the Fermi surface momentum. Pion-less $\Delta$ decay is also taken into account, where $20 \%$ of the events do not have a pion and only the lepton and nucleon are generated [73].

\subsubsection{Coherent pion production}

The model of Rein and Sehgal [31,34], which is widely used in neutrino oscillation experiments, is used to simulate coherent pion production in the NEUT simulation. Based on Adler's PCAC theorem, the differential cross section for $Q^{2}=0$ is expressed as

$$
\left.\frac{d^{3} \sigma(\nu A \rightarrow \ell A \pi)}{d x d y d t}\right|_{Q^{2}=0}=\left.\frac{G_{F}^{2}}{\pi^{2}} f_{\pi}^{2} m_{N} E_{\nu}(1-y) \frac{\sigma(\pi A \rightarrow \pi A)}{d t}\right|_{E_{\nu} y=E_{\pi}},
$$

where $x=Q^{2} / 2 m_{N} \nu$ and $y=\nu / E_{\nu}$ are the Bjorken kinematic variables, $\nu$ is the energy transfer, $t$ is the square of the four-momentum transfered to the nucleus, $G_{F}$ is the weak 
coupling constant, $f_{\pi}$ is the pion decay constant, and $m_{N}$ is the mass of nucleon. The relation expresses the forward neutrino cross section at the nucleus $A$ in terms of the cross section for the process $\pi A \rightarrow \pi A$.

The extrapolation of the cross section to $Q^{2} \neq 0$ is performed based on the method of hadron dominance, which is an extension of the model used in electromagnetic interactions, the vector meson dominance (VMD) model. The VMD model treats the electromagnetic current as a superposition of the contribution from the lightest vector mesons as

$$
\mathcal{A}(\gamma+X \rightarrow Y)=\sum_{V=\rho, \omega, \phi} \frac{e}{g_{V}} \frac{m_{V}^{2}}{Q^{2}+m_{V}^{2}} \mathcal{A}(V+X \rightarrow Y) .
$$

In case of weak interaction, the $\rho$ meson is used for the vector current, and the $\pi$ and $a_{1}$ mesons are used for the axial vector current. For coherent pion production, the axial vector current contribution is considered to be dominant, and the contributions of the vector current and the interference between the vector and axial-vector currents are thought to be negligible. Following the calculation of the VMD model, the cross section is then obtained by attaching a propagator term:

$$
\frac{d^{3} \sigma(\nu A \rightarrow \ell A \pi)}{d x d y d t}=\left.\frac{G_{F}^{2}}{\pi^{2}} f_{\pi}^{2} m_{N} E_{\nu}(1-y)\left(\frac{m_{A}^{2}}{Q^{2}+m_{A}^{2}}\right)^{2} \frac{\sigma(\pi A \rightarrow \pi A)}{d t}\right|_{E_{\nu} y=E_{\pi}}
$$

where $m_{A}$ is the axial vector mass, introduced practically in the propagator.

The pion-nucleus differential cross section is expressed as

$$
\frac{d \sigma(\pi A \rightarrow \pi A)}{d t}=\left.A^{2}\left|F_{A}(t)\right|^{2} \frac{d \sigma(\pi N \rightarrow \pi N)}{d t}\right|_{t=0}
$$

where $A$ is the atomic number of the nucleus, $F_{A}(t)$ is the nuclear form factor (including the effect of pion absorption). With the aid of the optical theorem, the pion-nucleon differential cross section in the forward direction is given by

$$
\left.\frac{d \sigma(\pi N \rightarrow \pi N)}{d t}\right|_{t=0}=\frac{1}{16 \pi}\left[\sigma_{\mathrm{tot}}^{\pi N}\right]^{2}\left(1+r^{2}\right), \quad r=\frac{\operatorname{Re} f_{\pi N}(0)}{\operatorname{Im} f_{\pi N}(0)} .
$$

In the model, an average cross section from measurements of pion-deuteron scattering is incorporated as $\sigma_{\text {tot }}^{\pi N}$. For the nuclear form factor, a simple form of

$$
\left|F_{A}(t)\right|^{2}=e^{-b|t|} F_{\text {abs }}
$$

is adopted, where $b$ is related to the nuclear radius $R$, given by

$$
b=\frac{1}{3} R^{2} \quad\left(R=R_{0} A^{1 / 3}\right) .
$$

The term $F_{\text {abs }}$ is a $t$-independent attenuation factor representing the effect of pion absorption in the nucleus, expressed as

$$
F_{\mathrm{abs}}=e^{-\langle x\rangle / \lambda},
$$

where $\langle x\rangle$ and $\lambda$ are the average path length traversed by the pion produced in the nucleus, and the absorption length, respectively. By assuming the nucleus is a homogeneous sphere with an uniform density, $\langle x\rangle$ and $\lambda$ are calculated as

$$
\langle x\rangle=\frac{3}{4} R, \quad \lambda=A\left(\frac{4 \pi R^{3}}{3}\right)^{-1} \sigma_{\text {inel }}^{\pi N},
$$


where $\sigma_{\text {inel }}^{\pi N}$ is the pion-nucleon inelastic cross section. This leads to

$$
F_{\text {abs }}=\exp \left(-\frac{9 A^{1 / 3}}{16 \pi R_{0}^{2}} \sigma_{\text {inel }}^{\pi N}\right)
$$

Therefore, the differential cross section for coherent pion production in the model of Rein and Sehgal is expressed as

$$
\frac{d^{3} \sigma(\nu A \rightarrow \ell A \pi)}{d x d y d t}=\frac{G_{F}^{2}}{\pi^{2}} f_{\pi}^{2} m_{N} E_{\nu}(1-y)\left(\frac{m_{A}^{2}}{Q^{2}+m_{A}^{2}}\right)^{2} \frac{A^{2}}{16 \pi}\left[\sigma_{\text {tot }}^{\pi N}\right]^{2}\left(1+r^{2}\right) e^{-b|t|} F_{\text {abs }} .
$$

The Rein and Sehgal formalism predicts the following features of coherent pion production.

1. Since the axial parts of the neutral and charged currents form a triplet in isospace, we are led to $f_{\pi^{0}}^{2}=\left(\sqrt{1 / 2} f_{\pi^{+}}\right)^{2}=(1 / 2) f_{\pi^{+}}^{2}=(1 / 2)\left(0.93 m_{\pi}\right)^{2}$. Therefore, the model predicts the relation between the neutral and charged current coherent pion production cross sections as $\sigma(\mathrm{CC})=2 \times \sigma(\mathrm{NC})$. The relation is slightly modified by the lepton mass correction described below.

2. The $A$ dependence of the cross section turns out to be approximately $A^{1 / 3}$. The behavior results roughly from a product $A^{4 / 3} b^{-1} b^{-1 / 2}$, where $b \sim R^{2} \sim A^{2 / 3}$. The first factor is the effect of nuclear coherence, including the effects of pion absorption, the second factor comes from integration of $e^{-b|t|}$ over $t$, the third one comes from integration over $x$.

3. The model predicts the same cross section for coherent pion production by neutrinos and antineutrinos.

There is an important modification when the mass of out-going lepton is taken into account. This modification is expressed as a simple multiplicative correction factor;

$$
C=\left(1-\frac{1}{2} \frac{Q_{\min }^{2}}{Q^{2}+m_{\pi}^{2}}\right)^{2}+\frac{1}{4} y \frac{Q_{\min }^{2}\left(Q^{2}-Q_{\min }^{2}\right)}{\left(Q^{2}+m_{\pi}^{2}\right)^{2}},
$$

where

$$
Q_{\min }^{2}=m_{\ell}^{2} \frac{y}{1-y}
$$

The range of the variable $Q^{2}$ is

$$
Q_{\min }^{2}<Q^{2}<2 m_{N} E_{\nu} y_{\max }
$$

where $y$ lies between $y_{\min }=m_{\pi} / E_{\nu}$ and $y_{\max }=1-m_{\ell} / E_{\nu}$. Thus, the differential cross section with lepton mass correction is expressed as

$$
\left.\frac{d^{3} \sigma}{d x d y d z}\right|_{m_{\ell} \neq 0}=\left.\frac{d^{3} \sigma}{d x d y d z}\right|_{m_{\ell}=0} \cdot C \theta\left(Q^{2}-Q_{\min }^{2}\right) \theta\left(y-y_{\min }\right) \theta\left(y_{\max }-y\right) .
$$

The lepton mass correction is applied only to the charged current channel, and the neutral current channel is unaffected.

In our MC simulation, we set the axial vector mass, $m_{A}$, to $1.0 \mathrm{GeV} / c^{2}$, and the nuclear radius parameter, $R_{0}$, is set to $1.0 \mathrm{fm}$. For the total and inelastic pion-nucleon cross sections, $\sigma_{\text {tot }}^{\pi N}$ and $\sigma_{\text {inel }}^{\pi N}$, respectively, the fitted results given in Rein and Sehgal's paper are employed. 


\subsubsection{Deep inelastic scattering (DIS)}

The double differential cross section for charged current deep inelastic scattering (DIS) is calculated as:

$$
\begin{aligned}
\frac{d^{2} \sigma}{d x d y} & =\frac{G_{F}^{2} m_{N} E_{\nu}}{\pi}\left[\left(1-y+\frac{1}{2} y^{2}+C_{1}\right) F_{2}(x) \pm y\left(1-\frac{1}{2} y+C_{2}\right) x F_{3}(x)\right] \\
C_{1} & =\frac{m_{\ell}^{2}(y-2)}{4 m_{N} E_{\nu} x}-\frac{m_{N} x y}{2 E_{\nu}}-\frac{m_{\ell}^{2}}{4 E_{\nu}^{2}} \\
C_{2} & =-\frac{m_{\ell}^{2}}{4 m_{N} E_{\nu} x}
\end{aligned}
$$

where $x \equiv Q^{2} /\left(2 m_{N}\left(E_{\nu}-E_{\ell}\right)+m_{N}^{2}\right)$ and $y \equiv\left(E_{\nu}-E_{\ell}\right) / E_{\nu}$ are the Bjorken scaling parameters, and $E_{\ell}$ is the energy of the final state lepton. The nucleon structure functions, $F_{2}$ and $x F_{3}$, are calculated using the GRV98 parton distribution functions [74]. Additionally, we have included the corrections in the small $Q^{2}$ region developed by Bodek and Yang [75]. In the calculation, the hadronic invariant mass, $W$, is required to be larger than $1.3 \mathrm{GeV} / c^{2}$.

The multi-hadron final states are simulated with two models. In the range of $1.3<$ $W<2.0 \mathrm{GeV} / c^{2}$, a custom-made program [76] is employed. The mean multiplicity of pions, $\left\langle n_{\pi}\right\rangle$, is estimated from the experimental result [77], assuming $\left\langle n_{\pi^{+}}\right\rangle=\left\langle n_{\pi^{-}}\right\rangle=$ $\left\langle n_{\pi^{0}}\right\rangle$, to be

$$
\left\langle n_{\pi}\right\rangle=0.09+1.83 \ln W^{2}
$$

The number of pions for each event is determined using KNO (Koba-Nielsen-Olesen) scaling [78]. Since single pion production is already included in the simulation, the multiplicity of pions is restricted to be larger than or equal to two in this $W$ region. The forward-backward asymmetry of pion multiplicity in the hadronic center-of-mass system, estimated from the experimental result [79], is also taken into account:

$$
\frac{\left\langle n_{\pi}^{F}\right\rangle}{\left\langle n_{\pi}^{B}\right\rangle}=\frac{0.35+0.41 \ln W^{2}}{0.50+0.09 \ln W^{2}}
$$

For the events with $W>2 \mathrm{GeV} / c^{2}$, PYTHIA/JETSET [80] is used to calculate the kinematics of hadronic final states.

To obtain the cross sections for neutral current deep inelastic scattering, we use the following relations:

$$
\begin{aligned}
& \frac{\sigma(\nu \text { NC-DIS })}{\sigma(\nu \text { CC-DIS })}= \begin{cases}0.26 & \left(E_{\nu} \leq 3 \mathrm{GeV}\right) \\
0.26+0.04 \times\left(E_{\nu} / 3-1\right) & \left(3<E_{\nu}<6 \mathrm{GeV}\right) \\
0.30 & \left(E_{\nu} \geq 6 \mathrm{GeV}\right)\end{cases} \\
& \frac{\sigma(\bar{\nu} \mathrm{NC}-\mathrm{DIS})}{\sigma(\bar{\nu} \mathrm{CC}-\mathrm{DIS})}= \begin{cases}0.39 & \left(E_{\nu} \leq 3 \mathrm{GeV}\right) \\
0.39-0.02 \times\left(E_{\nu} / 3-1\right) & \left(3<E_{\nu}<6 \mathrm{GeV}\right) \\
0.37 & \left(E_{\nu} \geq 6 \mathrm{GeV}\right)\end{cases}
\end{aligned}
$$

These relations are estimated from the experimental results $[81,82]$. 


\subsection{Intra-nuclear interactions}

The intra-nuclear interactions of mesons and nucleons produced in neutrino interactions in the nuclei are simulated. These interactions are treated using a cascade model, and each of the particles is traced until it escapes from the nucleus. The neutrino interaction position in the nucleus is calculated using the Wood-Saxon type nucleon density distribution:

$$
\rho(r)=\frac{Z}{A} \rho_{0}\left[1+\exp \left(\frac{r-c}{a}\right)\right]^{-1},
$$

where $\rho_{0}=0.48 m_{\pi}^{3}, A$ and $Z$ are the mass number and atomic number of the nucleus, respectively. For carbon nucleus, $a=0.52 \mathrm{fm}$ and $c=2.36 \mathrm{fm}$ [83]. Fermi motion of nucleons in the nucleus and Pauli blocking effect are taken into account in the simulation. The Fermi surface momentum at the interaction point is defined as

$$
p_{F}(r)=\left(\frac{3}{2} \pi^{2} \rho(r)\right)^{\frac{1}{3}} .
$$

\subsubsection{Pion interactions}

Among all the interactions of mesons and nucleons, the interactions of pions are most important to these analyses. The inelastic scattering, charge exchange and absorption of pions in the nuclei are simulated. The interaction cross sections of pions in the nuclei are calculated using the model by Salcedo et al. [84], which agrees well with past experimental data [85]. If inelastic scattering or charge exchange occurs, the direction and momentum of pions are determined by using results from a phase shift analysis of pion-nucleus scattering experiments [86]. When calculating the pion scattering amplitude, Pauli blocking effect is taken into account by requiring the nucleon momentum after the interaction to be larger than the Fermi surface momentum at the interaction point.

In SciBooNE, approximately $15 \%$ of pions produced via neutrino interaction are absorbed in carbon, about $20 \%$ of pions are inelastically scattered, and the probability of charge exchange is $5 \%$.

\subsubsection{Nucleon re-scattering}

Re-interactions of the recoil protons and neutrons produced in neutrino interactions are also important, because the proton tracks are used to classify the neutrino event type. Nucleon-nucleon interactions modify the outgoing nucleon's momentum and direction. Both elastic scattering and pion production are considered. In order to simulate these interactions, the cascade model is again used and the generated particles in the nucleus are tracked using the same code as for the mesons. The differential cross sections were obtained from nucleon-nucleon scattering experiments [87]. For pion production, the isobaric nucleon model [88] is used.

In SciBooNE, approximately 35\% of protons produced via charged current quasi-elastic scattering interact in carbon. 


\section{Chapter 5}

\section{SciBooNE Detector}

Figure 5.1 shows a schematic drawing of the SciBooNE detector. The SciBooNE detector consists of three sub-detectors: a fully active and finely segmented scintillator bar tracker (SciBar), an electromagnetic calorimeter (EC) and a muon range detector (MRD). The SciBar and EC detectors are placed in a dark box. The dark box provides reference positions so that we can align SciBar and the EC, in addition to the main purpose of shielding light. In the following sections, sub-detectors and the data acquisition system are described. The detector coordinate and alignment of the detector are also discussed.

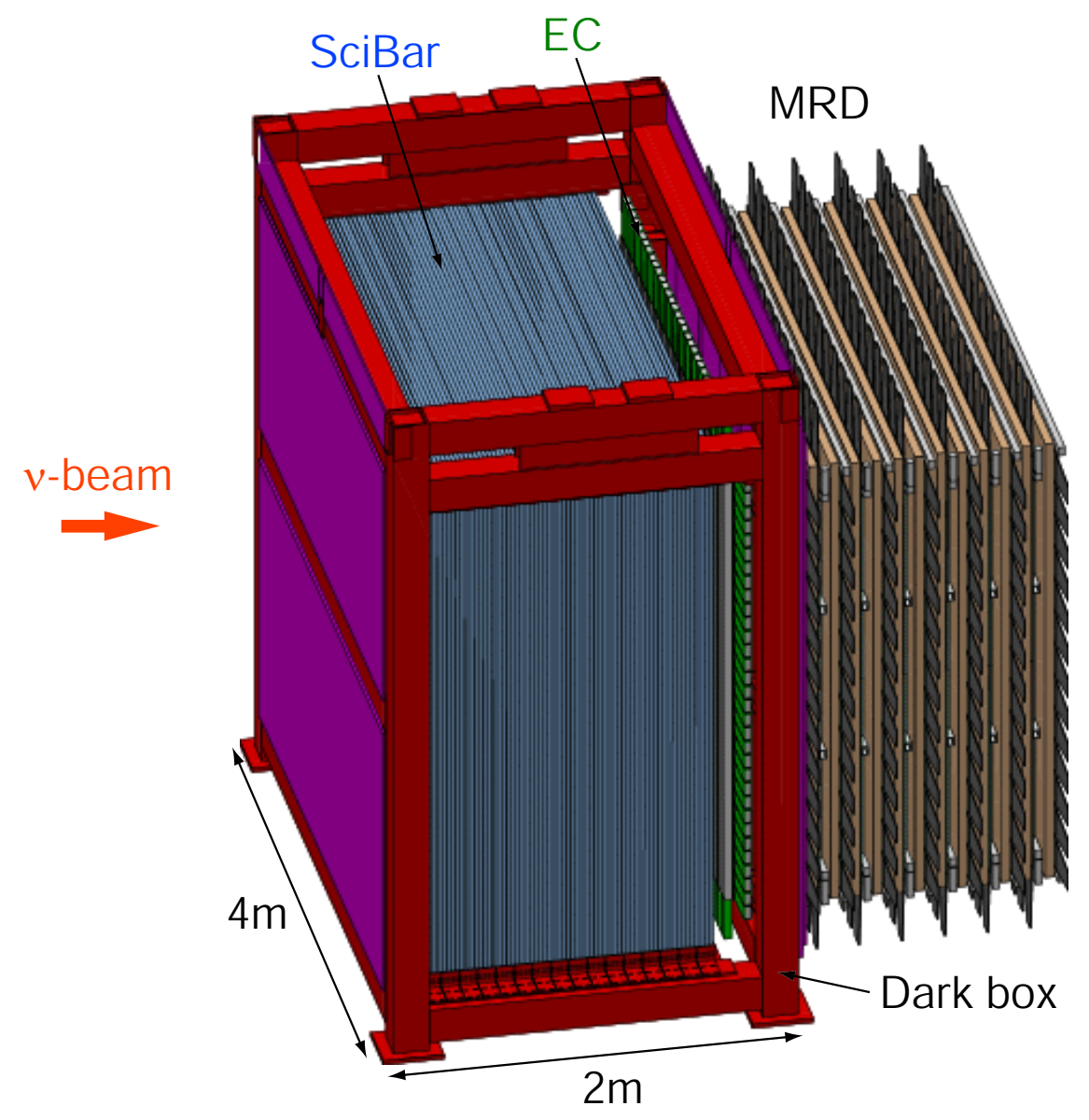

Figure 5.1: Schematic drawing of the SciBooNE detector. 


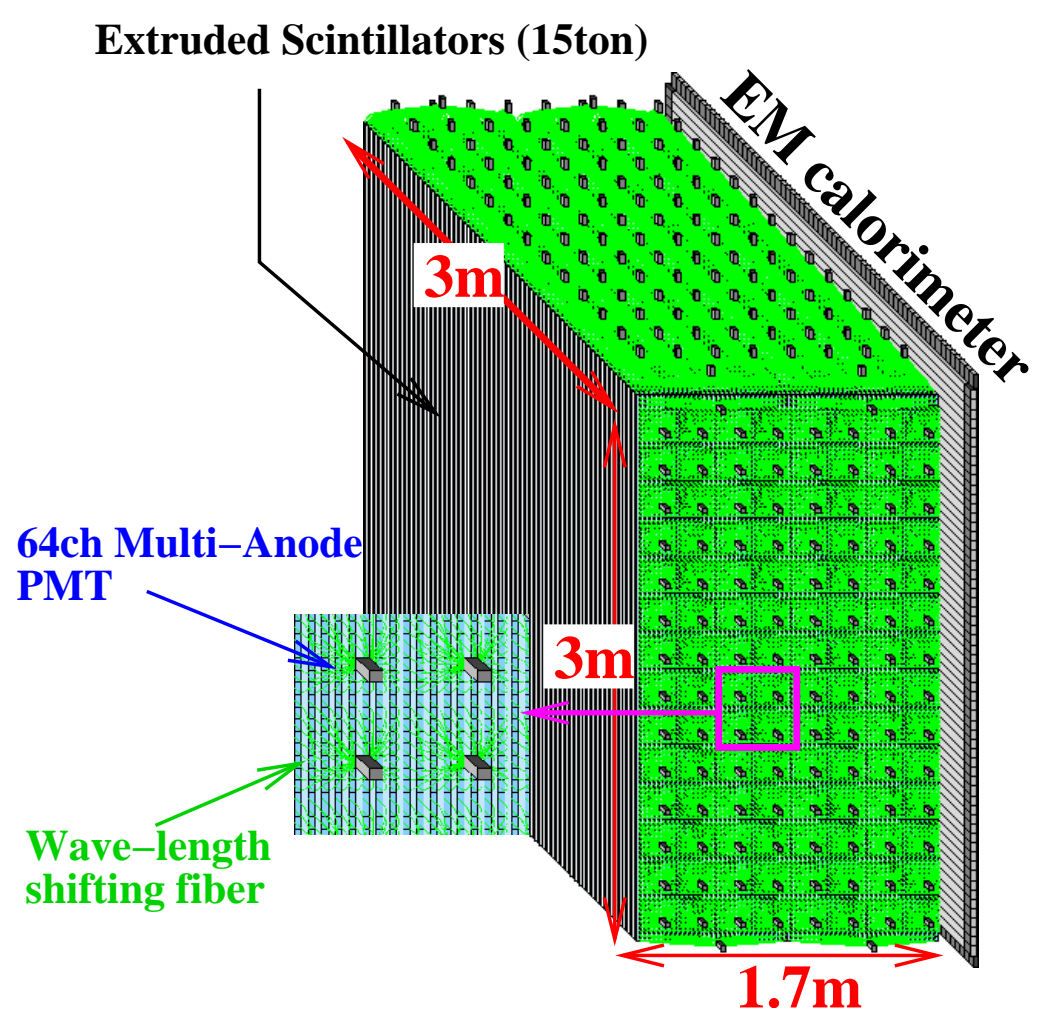

Figure 5.2: Schematic drawing of SciBar.

\subsection{Scintillator Bar Tracker (SciBar)}

The SciBar detector is positioned upstream of the other sub-detectors. The primary role of SciBar is to reconstruct the neutrino-nucleus interaction vertex and detect charged particles produced by neutrino interactions. Moreover, SciBar is capable of particle identification based on energy deposition per unit length. SciBar was originally designed and built as a near detector for the K2K experiment [50]. After K2K's completion, the SciBar detector was once disassembled, shipped to FNAL, and then re-built there for SciBooNE.

Figure 5.2 shows a schematic drawing of SciBar. The SciBar detector consists of 14,336 extruded plastic scintillator strips which serve as the target for the neutrino beam as well as the active detection medium. Each strip has dimensions of $1.3 \times 2.5 \times 300 \mathrm{~cm}^{3}$. The scintillators are arranged vertically and horizontally to construct a $3 \times 3 \times 1.7 \mathrm{~m}^{3}$ volume with a total mass of 15 tons. Each strip is read out by a wavelength shifting (WLS) fiber attached to a 64-channel multi-anode photomultiplier tube (MA-PMT) as shown in Figure 5.3. Charge and timing information from each MA-PMT is recorded by a front-end electronics board (FEB) attached directly to the MA-PMT and a back-end VME module. The stability of the gain of the MA-PMTs is monitored by a custom-made monitoring system using light-emitting diodes (LEDs). The energy and timing calibrations are done by using cosmic-ray muons.

Table 5.1 summarizes specifications of the SciBar detector. In the following sections, we describe each component in detail and show the basic performance of the detector. 
Table 5.1: Specifications of the SciBar detector

\begin{tabular}{|c|c|}
\hline \multicolumn{2}{|r|}{ Structure } \\
\hline Dimensions & $3 \mathrm{~m} \times 3 \mathrm{~m} \times 1.7 \mathrm{~m}$ \\
\hline Weight & 15 tons \\
\hline Number of channels & 14,336 \\
\hline \multicolumn{2}{|r|}{ Scintillator } \\
\hline Material & Polystyrene, PPO(1\%), POPOP $(0.03 \%)$ \\
\hline Emission peak wavelength & $420 \mathrm{~nm}$ \\
\hline Reflector material & $\mathrm{TiO}_{2}(15 \%)$ infused in polystyrene \\
\hline Dimensions & $1.3 \mathrm{~cm} \times 2.5 \mathrm{~cm} \times 300 \mathrm{~cm}$ \\
\hline Density & $1.021 \mathrm{~g} / \mathrm{cm}^{3}$ \\
\hline \multicolumn{2}{|r|}{ WLS fiber } \\
\hline Type & Kuraray Y11(200)MS, multi-clad \\
\hline Material & polystyrene(core), acrylic(inner), polyfluor(outer) \\
\hline Refractive index & 1.56 (core), 1.49 (inner), 1.42 (outer) \\
\hline Absorption peak wavelength & $430 \mathrm{~nm}$ \\
\hline Emission peak wavelength & $476 \mathrm{~nm}$ \\
\hline Diameter & $1.5 \mathrm{~mm}$ \\
\hline Attenuation length & $350 \mathrm{~cm}$ (typical) \\
\hline \multicolumn{2}{|r|}{ MA-PMT } \\
\hline Model & Hamamatsu H8804 \\
\hline Anode & $8 \times 8$ pixels (pixel size: $2 \times 2 \mathrm{~mm}^{2}$ ) \\
\hline Cathode & Bialkali (Sb-K-Cs) \\
\hline Sensitive wavelength & 300-650 nm (peak: $420 \mathrm{~nm})$ \\
\hline Quantum efficiency & $12 \%$ at $\lambda=500 \mathrm{~nm}$ \\
\hline Dynode & Metal channel structure, 12 stages \\
\hline Gain & typical $6 \times 10^{5}$ at $800 \mathrm{~V}$ \\
\hline Response linearity & $\begin{array}{l}\text { within } 10 \% \text { up to } 200 \text { photoelectrons } \\
\text { with the gain of } 6 \times 10^{5}\end{array}$ \\
\hline Crosstalk & $3.15 \%$ (adjacent pixel) \\
\hline \multicolumn{2}{|r|}{ Readout electronics } \\
\hline Number of ADC channels & 14,336 \\
\hline ADC pedestal width & below 0.3 photoelectron \\
\hline ADC response linearity & $\begin{array}{l}\text { within } 5 \% \text { up to } 300 \text { photoelectrons } \\
\text { with the gain of } 5 \times 10^{5}\end{array}$ \\
\hline Number of TDC channels & 448 \\
\hline TDC resolution & $0.78 \mathrm{nsec}$ \\
\hline TDC full range & $50 \mu \mathrm{sec}$ \\
\hline
\end{tabular}




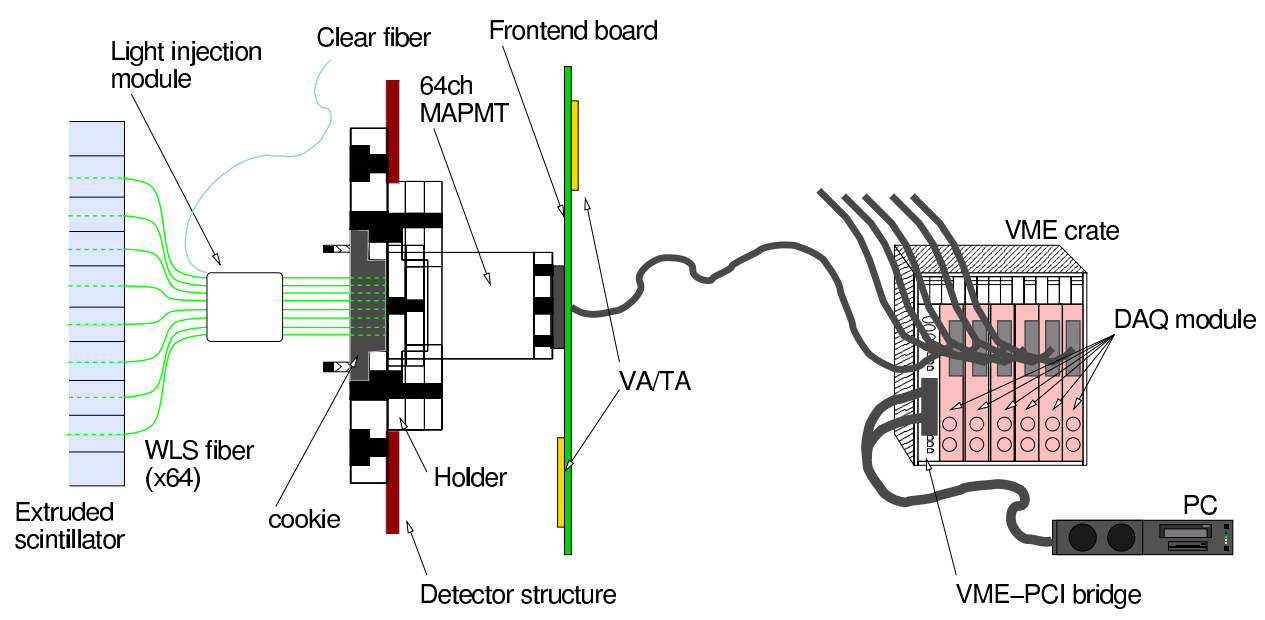

Figure 5.3: Schematic drawing of the SciBar readout system.

\subsubsection{Extruded scintillator}

The extruded scintillator strips are made of polystyrene $\left(\mathrm{C}_{8} \mathrm{H}_{8}\right)$, infused with the fluors PPO and POPOP ( $1 \%$ and $0.03 \%$ by weight, respectively), with an emission peak wavelength of $420 \mathrm{~nm}$. The scintillator is developed and produced by FNAL, and its composition is same as that of the scintillator used in the MINOS experiment at FNAL.

Figure 5.5 shows a schematic drawing of the scintillator strip. The scintillator is $2.5 \mathrm{~cm}$ wide, $1.3 \mathrm{~cm}$ thick, and $300 \mathrm{~cm}$ long. There is a $1.8 \mathrm{~mm}$ diameter hole in the center to insert the WLS fiber. The scintillator is covered with co-extruded reflective coating, composed of $\mathrm{TiO}_{2}$ infused in polystyrene ( $15 \%$ by weight). The dimensions and weight of the strip were measured by sampling of $10 \%$ of the total. The averaged density of the scintillator is $1.021 \mathrm{~g} / \mathrm{cm}^{3}$, estimated from the measured dimensions and weight. The scintillator array of SciBar consists of 64 layers along the beam axis. Each layer is comprised of 112 vertical strips and 112 horizontal strips, and the vertical and horizontal planes are glued together with an aluminium frame using epoxy. Each layer module has a dimension of $300 \times 300 \times 2.6 \mathrm{~cm}^{3}$, mounted individually onto the dark box.

The light yield from the scintillator is not in general proportional to the energy given by the ionization process. There is a reduction of the light yield at larger energy deposition, and thus the effect is called scintillator quenching. The relation between the visible energy $\Delta E_{\mathrm{vis}}$ and the actual deposited energy $\Delta E$ is expressed by Birk's law [89];

$$
\frac{\Delta E_{\mathrm{vis}}}{\Delta E} \propto \frac{1}{1+c \cdot d E /\left.d x\right|_{\exp }}
$$

where $d E /\left.d x\right|_{\text {exp }}$ is the expected energy deposition per unit length, and $c$ is Birk's constant which depends on material. The Birk's constant for the SciBar scintillator is measured to be $0.0208 \pm 0.0023 \mathrm{~cm} / \mathrm{MeV}$ [90], using a prototype of SciBar in a proton beam (Figure 5.6).

\subsubsection{Wavelength shifting (WLS) fiber}

WLS fibers, Kuraray Y11(200)MS type, are used to collect the scintillation light for readout. Figure 5.7 shows the absorption and emission spectra of the WLS fiber. The 


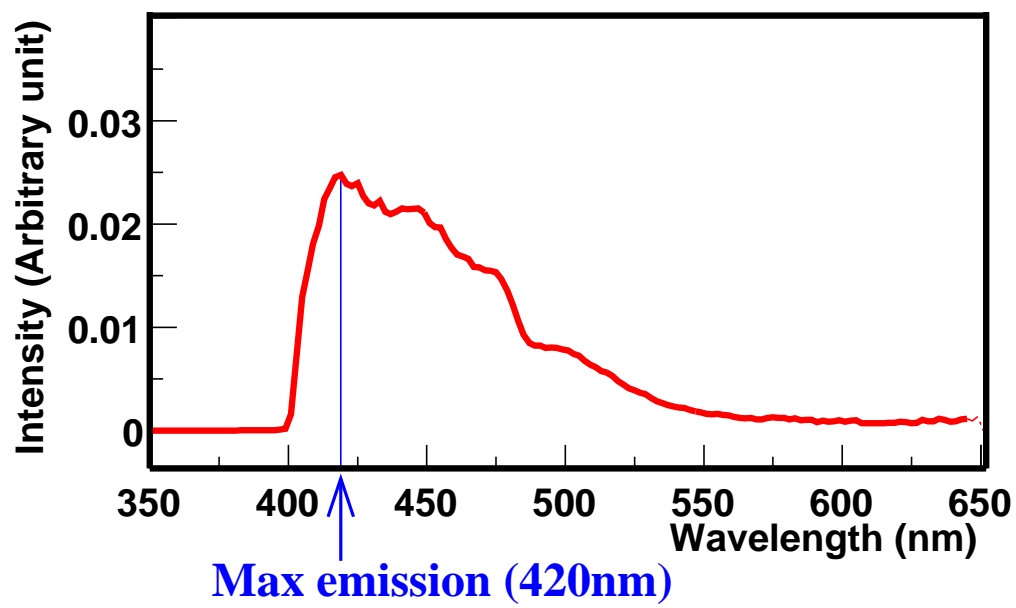

Figure 5.4: Emission spectrum for the SciBar scintillator (Ref. [91]).

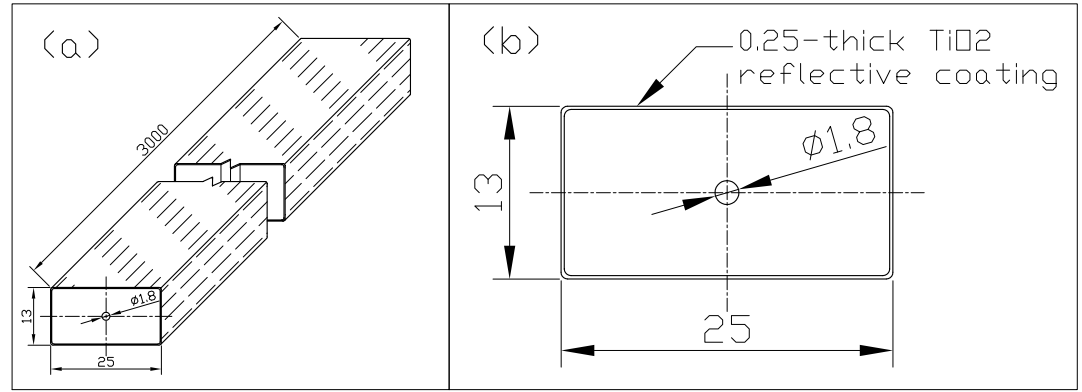

Figure 5.5: Schematic drawing of a scintillator strip. (a) Three-dimensional view and (b) Cross section. The unit is $\mathrm{mm}$.

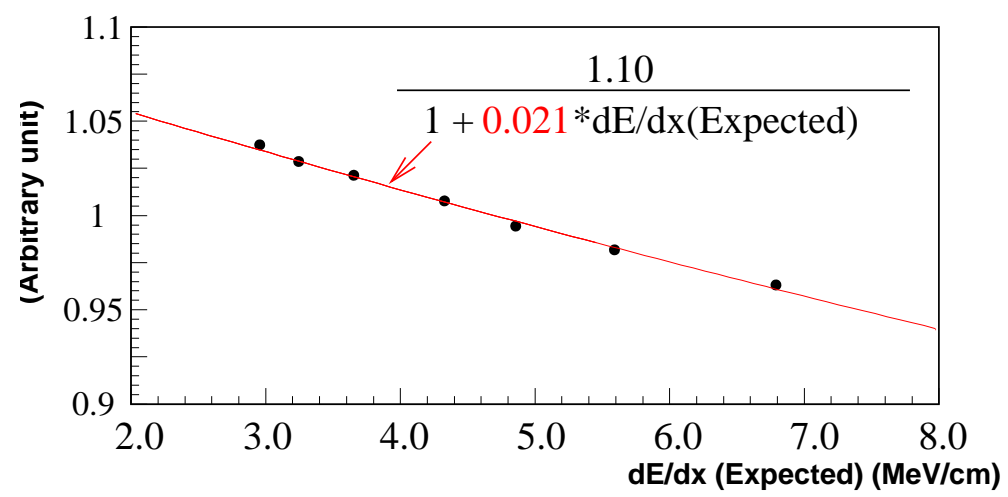

Figure 5.6: Ratio of the observed energy deposition per unit length $(d E / d x)$ to the expected $d E / d x$ as a function of the expected $d E / d x$, measured with a prototype of SciBar in a proton beam. 


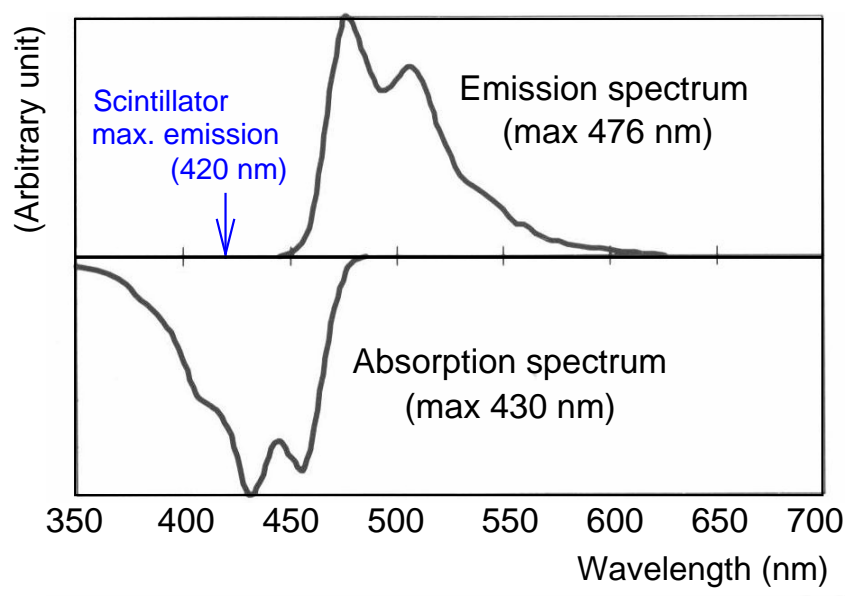

Figure 5.7: Absorption and emission spectra of Kuraray Y11(200)MS type WLS fiber.

absorption peak wavelength of $430 \mathrm{~nm}$ matches with the peak scintillation wavelength of $420 \mathrm{~nm}$. The emission peak wavelength is $476 \mathrm{~nm}$.

Each fiber has a polystyrene core (reflective index $n=1.56$ ) which contains the wavelength shifting fluor with a concentration of $200 \mathrm{ppm}$, a thin acrylic inner clad $(n=1.49)$, and a thin polyfluor outer clad $(n=1.42)$. The diameter of the fiber is $1.5 \mathrm{~mm}$, and the length is $335 \mathrm{~cm}$ for vertical fibers and $360 \mathrm{~cm}$ for horizontal fibers. Sixty-four fibers are bundled together using a custom-made alignment fixture. All the fibers are aligned to pixels of MA-PMT with a precision of $0.2 \mathrm{~mm}$. In total, 224 fiber bundles are used in SciBar.

The attenuation length of all the WLS fibers were measured prior to installation in $\mathrm{K} 2 \mathrm{~K}$ [92]. The averaged attenuation length is $350 \mathrm{~cm}$. We checked the attenuation length before installation in SciBooNE, three years after production, by sampling of $4 \%$ of the total. The measured attenuation length is approximately $2 \%$ shorter in average than that measured before installation in $\mathrm{K} 2 \mathrm{~K}$, but no serious degradation is found.

\subsubsection{4-channel multi-anode PMT (MA-PMT)}

The scintillation light is detected by Hamamatsu H8804 MA-PMTs. Each MA-PMT has 64 channels, whose pixel size is $2 \mathrm{~mm} \times 2 \mathrm{~mm}$, arranged in an $8 \times 8$ array. The photocathode is made of bialkali (Sb-K-Cs), with a quantum efficiency of $12 \%$ at a wavelength of $500 \mathrm{~nm}$.

The gains of all MA-PMT channels were measured prior to installation in K2K [93]. The pixel-to-pixel gain uniformity is measured to be approximately $20 \%$ in RMS. The operation high voltage of each MA-PMT, typically $800 \mathrm{~V}$, is determined so that the averaged gain of 64 channels is $6 \times 10^{5}$. The absolute gain is measured from the single photoelectron (p.e.) peak. The temperature coefficient of the gain is measured to be $0.3 \%$ /degree C. The response linearity is kept within $10 \%$ up to 200 p.e. with the gain of $6 \times 10^{5}$ [94]. The single photoelectron resolution of the MA-PMT is $50 \pm 20 \%$, determined using cosmic-ray muons to reproduce the photoelectron distribution in the MC simulation.

The crosstalk effect, which is mainly caused by spreading of the incident light at the surface of photocathode, is measured in the laboratory. The amount of the crosstalk is 


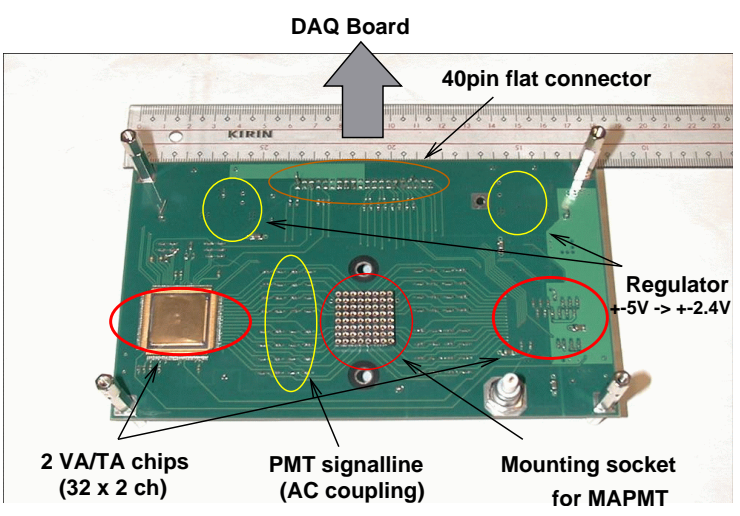

Figure 5.8: Picture of a front-end board.

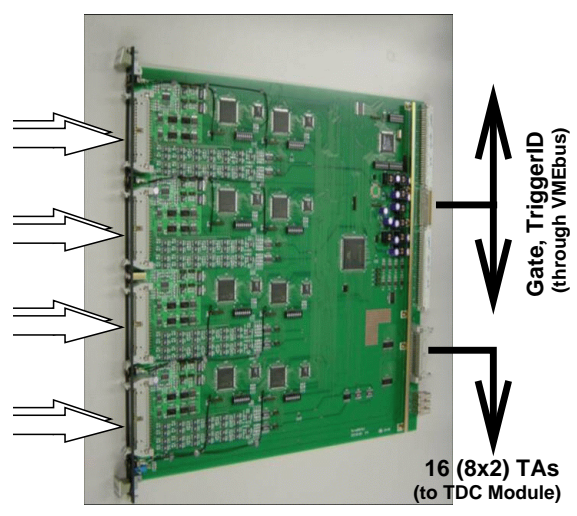

Figure 5.9: Picture of a DAQ board.

measured to be $3.15 \pm 0.4 \%$ for an adjacent channel, and $0.7 \%$ for an diagonal channel. The crosstalk to a next-to-next channel is between $0.1 \%$ and $0.3 \%$.

\subsubsection{Readout electronics}

The readout electronics system, originally developed for the K2K experiment [95], consists of a front-end electronics board (FEB) attached to each MA-PMT and a backend VME module. Figure 5.8 shows a picture of the FEB. On the FEB, a combination of VA and TA ASICs (IDEAS VA32HDR11 and TA32CG) is employed to multiplex pulseheight information from each anode of the MA-PMT and to make a fast-triggering signal. The VA has a 32-channel preamplifier-shaper circuit with a multiplexer. The slow shaper shapes the output with a peaking time of $1.2 \mu \mathrm{sec}$. The signal from each VA shaper is sampled at the time of an external hold request, and the result is passed to the multiplexer. The signal after preamplification in the VA is also sent to a fast shaper in the TA with a peaking time of 80 nsec. A logical "OR" of 32 channels is sent out from the TA. The intrinsic time jitter of the discriminated output is less than 1 ns. Each FEB has two packages of VA/TA, processing 64-channel charge information and two-channel timing information for each MA-PMT.

The back-end VME module, called the DAQ board, is developed as a standard VME$9 \mathrm{U}$ board. Figure 5.9 shows a picture of the DAQ board. Each DAQ board controls the readout of eight FEBs, and thus 28 DAQ boards are used in total. Each of the eight channels has line drivers to control front-end ASICs and a 12-bit flash analog-to-digital converter $(\mathrm{ADC})$ to digitize the multiplexed analog signal from the FEB with a 1-MHz readout clock. The readout system achieves low noise; the typical pedestal width is below 0.3 photoelectron. The linearity of the ADC response is kept within $5 \%$ up to $24 \mathrm{pC}$ which corresponds to 300 p.e. at the gain of $5 \times 10^{5}$ [94]. Timing information is sent to a 64channel multi-hit time-to-digital converter (TDC). The module was originally developed for the ATLAS experiment [96]. The timing resolution and full range are $0.78 \mathrm{nsec}$ and $50 \mu \mathrm{sec}$, respectively.

The TA signal is also sent to a cosmic-ray trigger board. The board is a general purpose logic board powered by an FPGA, and programmed to generate a signal when a cosmic-ray penetrates almost all the layers of SciBar. The signal is used for a cosmic-ray trigger for SciBar and the EC. 


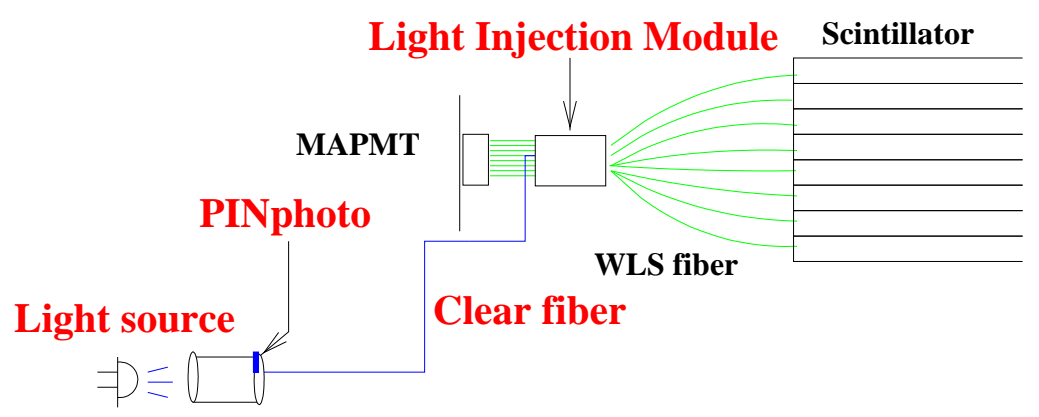

Figure 5.10: Schematic drawing of the SciBar gain monitoring system.

\subsubsection{Gain monitoring system}

In order to monitor and correct for the gain drift of all the MA-PMT channels continuously during data-taking, a custom-made gain monitoring system [90] is employed. ${ }^{1}$ The system consists of four sets of light sources, PIN photo-diodes, and clear fiber bundles. Figure 5.10 shows a schematic drawing of the SciBar gain monitoring system. A blue LED, NICHIA NSPB510, is used as the light source. Its emission spectrum (max: $460 \mathrm{~nm}$ ) matches with absorption spectrum of the WLS fiber. The LED is controlled by a custom-made LED driver. The driver, triggered by an external NIM pulse, provides a constant charge to the LED. The LED intensity is adjustable by changing the DC bias voltage to the driver, which is controlled by a 12-bit DAC (Digital-to-Analog Converter), BiRa Systems 5408. The PIN photo-diode, Hamamatsu S1227-101BQ, is used to monitor the intensity of the LED light. The photo-diode has a very good linearity up to $10^{9}$ photons, corresponding to $10^{3}$ p.e. for the MA-PMT. The photo-diodes are read out by an 8-channel 12-bit ADC, CAEN V265. The pulsed light from each LED is divided and carried to 56 MA-PMTs through clear fibers. A white cylinder, called a Light Injection Module, is assembled to the WLS fiber bundle in order to illuminate 64 WLS fibers uniformly. The LED light is absorbed by the WLS fibers, and then emitted light from the WLS fiber is carried to each channel of the MA-PMT.

Figure 5.11 shows the measured stability of the LED intensity during the whole datataking period. The LED intensity is monitored with a precision of better than $0.1 \%$. The intensity was stable to better than $2 \%$. Figure 5.12 shows the measured gain stability during the whole data-taking period for a typical MA-PMT channel. The relative gain is monitored every 8 hours with a precision of $0.1 \%$, and we correct for the gain drift. In the figure, we correct for the drift of the LED intensity monitored by the PIN photo-diode. The gain was stable within $\pm 2 \%$.

The number of dead channels is also monitored using the gain monitoring system. There was a certain period, corresponding to $1.5 \%$ of the total data-taking period, in which 16 channels did not respond to the LED light due to a problem of the readout electronics. However, during most of the period, only a few channels out of $14,336(0.03 \%$ of the total) were dead.

\footnotetext{
${ }^{1}$ The system was called HASE-moni (High Accuracy gain monitoring SystEm) in the K2K experiment.
} 


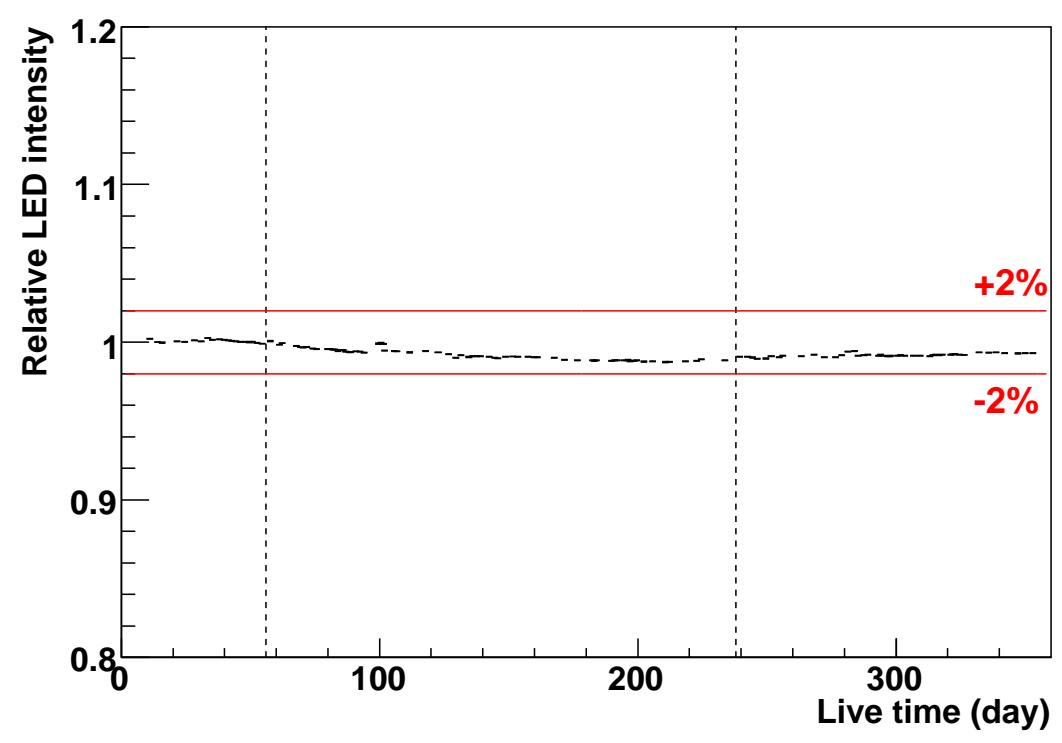

Figure 5.11: Measured stability of the LED intensity during the whole data-taking period.

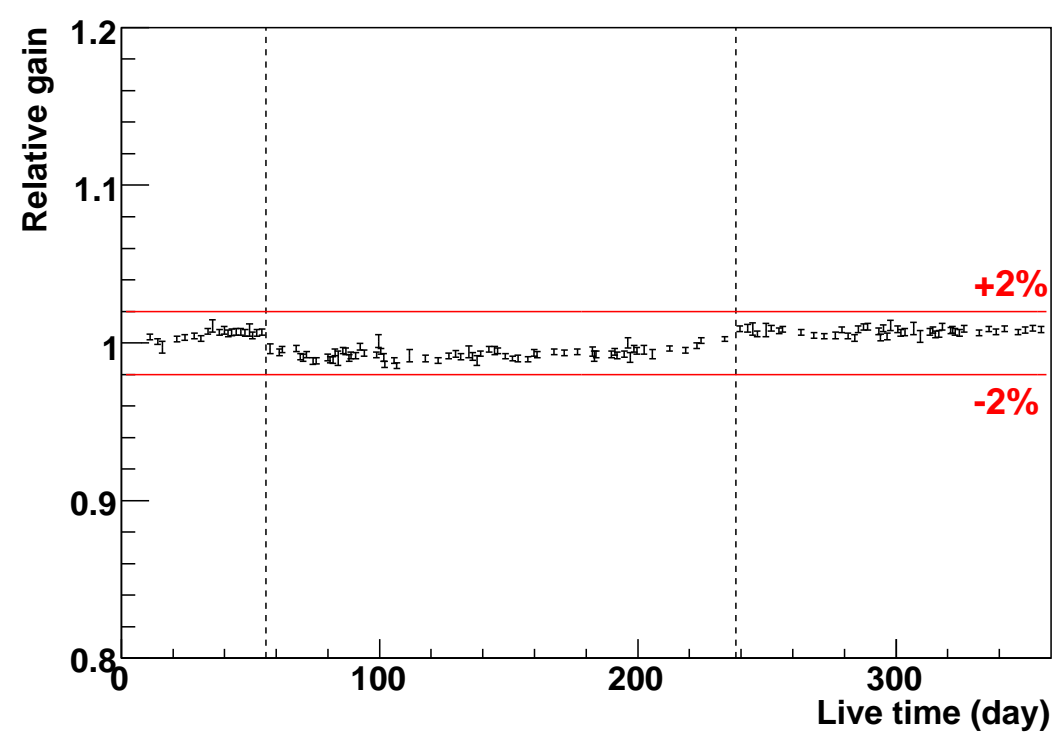

Figure 5.12: Measured gain stability during the whole data-taking period for a typical MA-PMT channel. 


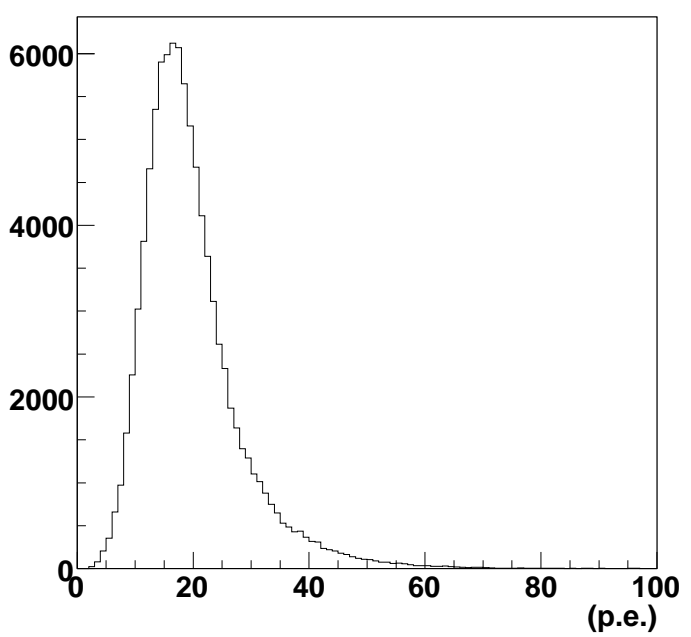

Figure 5.13: Number of photoelectrons for cosmic-ray muons for a typical channel.

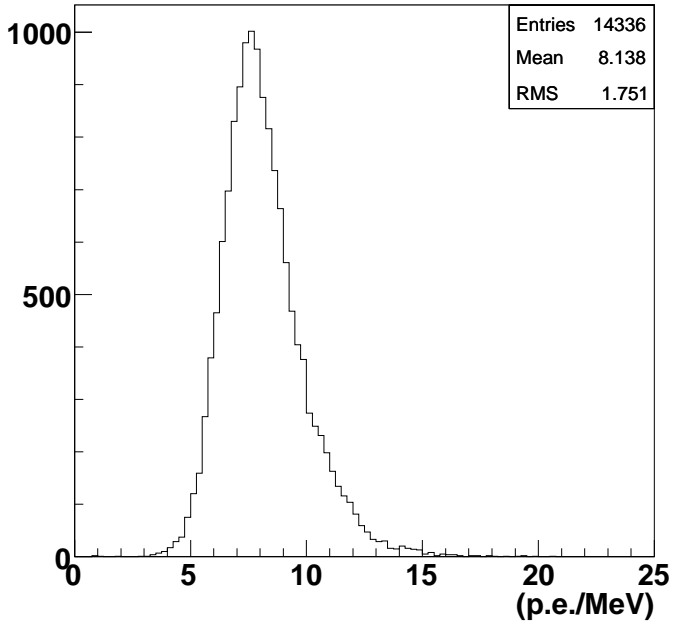

Figure 5.14: Energy calibration constants for all channels.

\subsubsection{Energy scale calibration}

The energy scale for each channel is calibrated with cosmic-ray muons. Figure 5.13 shows the number of photoelectrons for cosmic-ray muons for a typical channel. The path length of the particle inside the scintillator strip and the light attenuation in the WLS fiber are corrected in the figure. The averaged light yield for a minimum ionizing particle is measured to be approximately 20 p.e. per $1.3 \mathrm{~cm}$ path length. The energy calibration constant which converts the number of photoelectrons to the visible energy is measured for each channel. Figure 5.18 shows the energy calibration constants for all channels. The averaged value is 8.1 p.e./MeV, and the channel-by-channel variation is about $20 \%$.

\subsection{Electromagnetic Calorimeter (EC)}

The EC detector is a "spaghetti" type electromagnetic calorimeter, installed downstream of SciBar, and is designed to measure the electron neutrino contamination in the beam and tag photons from $\pi^{0}$ decay. The calorimeter modules were originally built for the CHORUS experiment at CERN [97] and later used in HARP and then K2K.

The calorimeter is made of modules of dimensions $262 \times 8.4 \times 4.2 \mathrm{~cm}^{3}$. The modules construct one vertical and one horizontal plane, and each plane has 32 modules. The planes cover an active area of $2.7 \times 2.6 \mathrm{~m}^{2}$. The EC has a thickness of 11 radiation lengths along the beam direction.

Each module consists of a stack of 21 lead sheets and 740 scintillating fibers. The $1 \mathrm{~mm}$ diameter scintillating fibers, Kuraray SCSF81, are embedded in the grooves on $1.9 \mathrm{~mm}$ thick lead sheets. The stack is kept together by a welded steel case. At each end of the module, fibers are grouped into two bundles, and each bundle is coupled to a Plexiglas light guide. The light guide is attached to 1 inch PMT, Hamamatsu R1335/SM, with a special green-extended photocathode. The cathode material is bialkali with an average quantum efficiency of $27 \%$ in the wavelength range of $350-450 \mathrm{~nm}$. A typical gain 


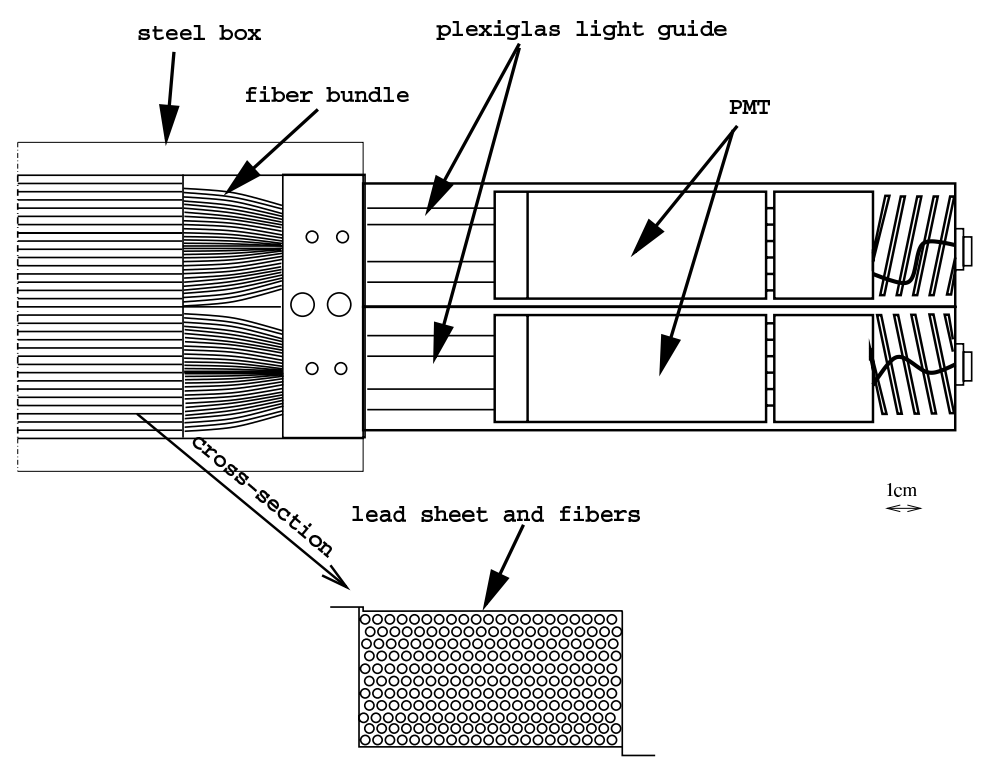

Figure 5.15: Schematic drawing of the EC module.

is $2 \times 10^{6}$ at the operation voltage of $1600 \mathrm{~V}$. In total, 256 PMTs are used in the EC. To select the spectral component with a larger attenuation length, a yellow filter (Kodak Wratten No.3) is inserted in front of the PMT. The attenuation length of the fiber is measured for each semi-module by using cosmic-ray muons to be approximately $400 \mathrm{~cm}$.

The PMTs produce a differential signal using the outputs from the cathode and the last dynode, and are read via multipolar differential screened cables which are approximately $100 \mathrm{~m}$ long. The readout system consists of eight 32-channel 12-bit QDC (Charge-toDigital Converter) modules, CAEN V792. Impedance matching cards, custom modified CAEN A992, are used to convert the $110 \Omega$ differential signals into $50 \Omega$ single ended signals and to decouple the PMT and QDC grounds.

The energy resolution for electrons was measured to be $14 \% / \sqrt{E(\mathrm{GeV})}$ using a test beam [97].

\subsection{Muon Range Detector (MRD)}

The MRD detector is installed downstream of the EC and is designed to measure the momentum of muons produced by charged-current neutrino interactions up to $1.2 \mathrm{GeV} / c$ using the observed range. The MRD was constructed for SciBooNE at FNAL, primarily out of parts recycled from past experiments.

The MRD consists of 12 iron plates and 13 alternating horizontal and vertical scintillator planes. Each iron plate is 2 inch thick, and covers an area of $274 \times 305 \mathrm{~cm}^{2}$. The total mass of absorber material is approximately 48 tons. The density of a spare iron plate was measured at several positions of the plate, to be $7.841 \pm 0.002 \mathrm{~g} / \mathrm{cm}^{3}$. The thickness of each plate was also measured prior to the experiment, with an accuracy of 1\%. The iron plates are sandwiched between scintillator planes. Each scintillator plane consists of $20 \mathrm{~cm}$ wide, $6 \mathrm{~mm}$ thick scintillator paddles. Each vertical scintillator plane is comprised of $138 \mathrm{~cm}$ long paddles, arranged in a $2 \times 15$ array to have an active area of 
Table 5.2: Specifications of the MRD detector.

\begin{tabular}{ll}
\hline \hline & \multicolumn{1}{c}{ Iron plate } \\
\hline Number of plates & 12 \\
Dimensions & $274 \times 305 \mathrm{~cm}^{2}, 2$ inch thickness \\
Density & Scintillator plane \\
\hline & 13 \\
\hline Number of planes & $2 \times 15$ (vertical), $13 \times 2$ (horizontal) \\
Segmentation & length: $138 \mathrm{~cm}$ (vertical), $155 \mathrm{~cm}$ (horizontal) \\
Dimensions of a counter & Phickness: $6 \mathrm{~mm}$, width: $20 \mathrm{~cm}$ \\
& EMI $9954 \mathrm{~KB}, 9839 \mathrm{~b}$ and $9939 \mathrm{~b}$ (horizontal) \\
\hline Model & Readout electronics \\
& 362 \\
\hline Number of channels & LeCroy $4300 \mathrm{~B}$ (ADC), Lecroy 3377 (TDC) \\
Model & 0.5 nsec \\
TDC resolution & $32 \mu$ sec \\
TDC full range &
\end{tabular}

$276 \times 300 \mathrm{~cm}^{2}$. On the other hand, each horizontal scintillator plane consists of $155 \mathrm{~cm}$ long paddles, arranged in a $13 \times 2$ array to have an active area of $260 \times 310 \mathrm{~cm}^{2}$. In total, 362 paddles are used in the MRD. The iron plates and scintillator paddles are recycled from the FNAL E605 experiment [98].

The scintillator paddles are read out by five types of 2 inch PMTs; the vertical planes consist of Hamamatsu 2154-05 PMTs from the NuTeV experiment and RCA 6342A PMTs, the horizontal planes consist of EMI 9954KB PMTs from the KTeV experiment, as well as EMI 9839b and 9939b PMTs. Charge and timing information from each PMT are recorded. The readout electronics system consists of LeCroy 4300B ADCs and LeCroy 3377 TDCs. The timing resolution and full range are $0.5 \mathrm{~ns}$ and $32 \mu \mathrm{sec}$, respectively. The energy threshold for TDC hits is approximately $250 \mathrm{keV}$ which corresponds to $20 \%$ of the signal from minimum ionizing particles. The single noise rate is typically $100 \mathrm{~Hz}$ for the horizontal planes, and is below $10^{4} \mathrm{~Hz}$ for the vertical planes.

The MRD has a cosmic-ray trigger independent from SciBar and the EC. Hit finding efficiency was continuously monitored using cosmic ray data taken between beam spills. Figure 5.16 shows the hit finding efficiency as a function of position for a typical scintillator plane. The average hit finding efficiency is $99 \%$.

\subsection{Data Acquisition (DAQ) System}

SciBooNE has two global triggers, the beam trigger and the off-beam trigger. Two types of data are collected in one beam cycle, neutrino data with the beam trigger and calibration data with the off-beam trigger. One cycle is about 2 sec which is defined by the accelerator timing sequence. The BNB receives one train of proton beam pulses per 


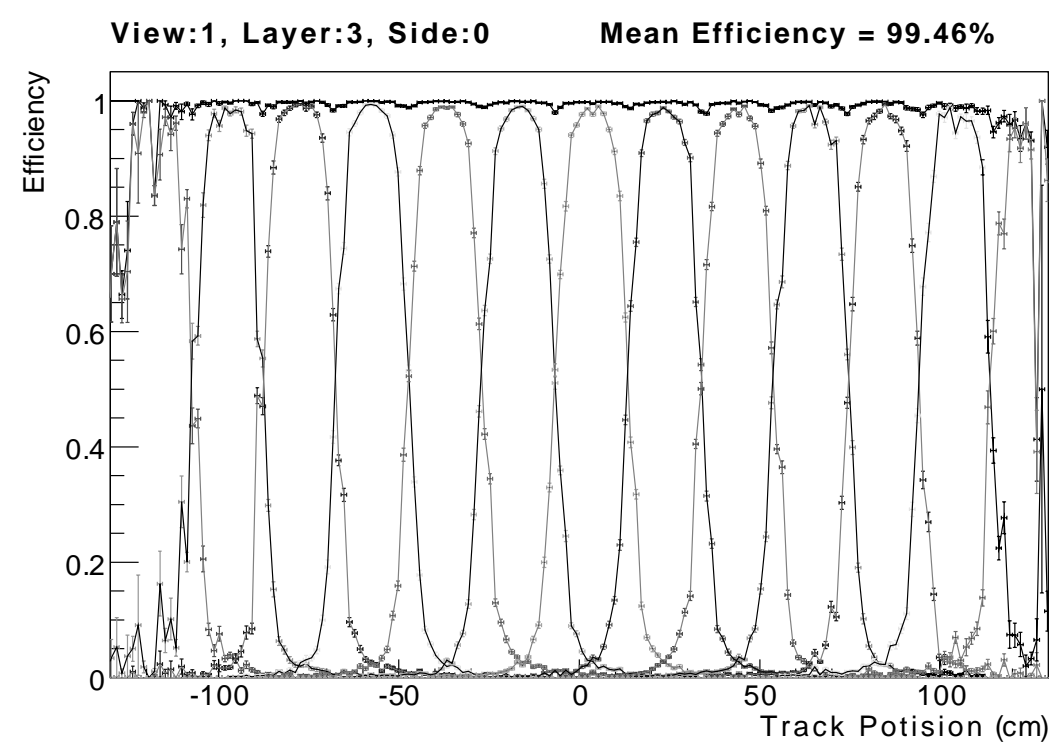

Figure 5.16: Hit finding efficiency as a function of position for a typical scintillator plane. Both individual counter efficiencies and the summed efficiency are shown.

cycle, with a maximum of 10 pulses in a row at $15 \mathrm{~Hz}$. A fast timing signal sent by the extraction magnet on BNB pulses establishes a beam-trigger. Once the beam trigger condition is set, all sub-detector systems read out all channels irrespective of hit occupancy (i.e. whether or not a neutrino interaction occurred), ensuring unbiased neutrino data. A Global Positioning System (GPS) is used to record the trigger timing. A PCI module, Symmetricom bc637 PCI-U, is connected to a Linux computer, and the GPS timing for each beam trigger is recorded. The accelerator information is provided by FNAL ACNET (Accelerator Control NETwork). The ACNET DAQ stream is independent from the detector DAQ stream, and the beam and detector information is merged at offline using the GPS time stamps.

After the beam trigger turns off, the off-beam trigger condition is automatically set and each sub-detector takes calibration data. There are three types of calibration data: pedestal, LED (only for SciBar) and cosmic ray data. The pedestal and LED data are collected once per cycle. For cosmic ray data, there are two independent trigger blocks: SciBar/EC and MRD. SciBar and the EC use a common cosmic ray trigger which is generated using fast signals from the TA. The MRD has its own cosmic ray trigger which is also self-generated by discriminator outputs. Both SciBar/EC and the MRD collect 20 cosmic ray triggers in a cycle.

\subsection{Detector Coordinate and Alignment}

\subsubsection{Detector coordinate}

Figure 5.17 shows an event display of a typical muon neutrino charged current quasielastic (CC-QE) scattering event candidate. The coordinate is shown in the figure. SciBooNE uses a right-handed Cartesian coordinate system in which the $z$ axis is the beam direction and the $y$ axis is the vertical upward direction. Thus, the $x$ axis is the hori- 


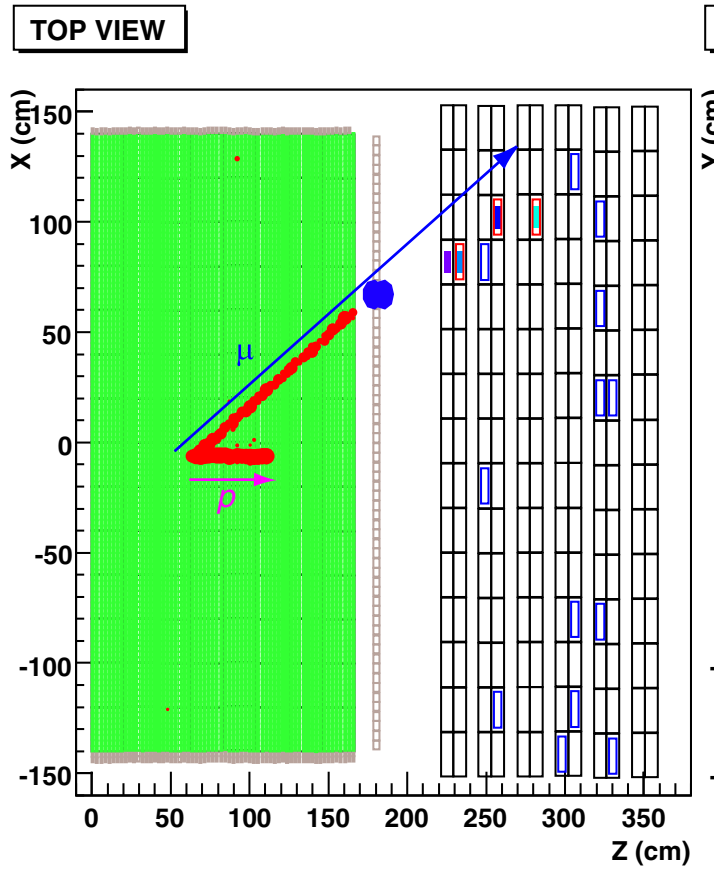

\section{SIDE VIEW}

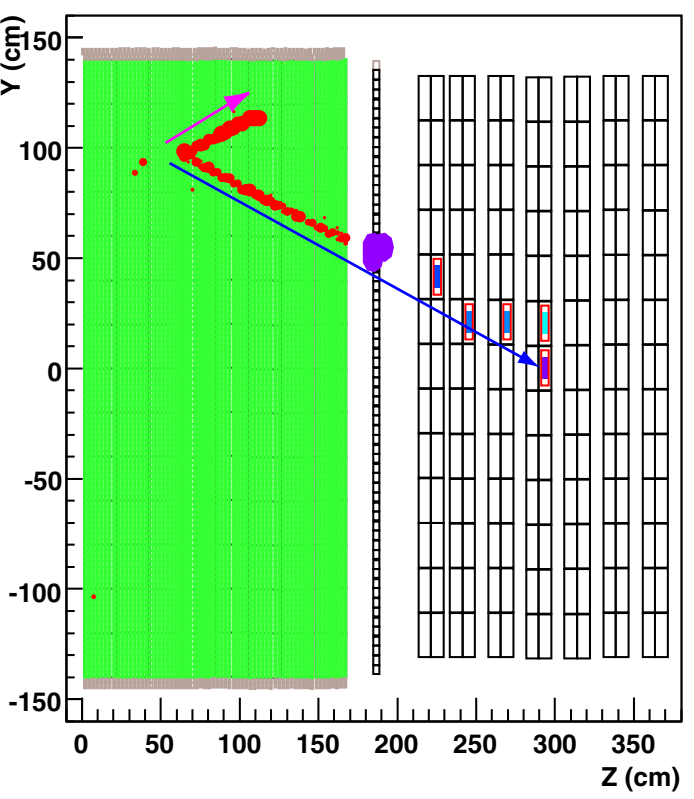

Figure 5.17: Event display of a typical muon neutrino charged current quasi-elastic scattering $\left(\nu_{\mu} n \rightarrow \mu^{-} p\right)$ candidate in SciBooNE data. Circles on SciBar indicate ADC hits, and the area of the circle is proportional to the energy deposition in the scintillator strip. Framed boxes on the MRD indicate TDC hits in the beam-on timing (red) and beam-off timing (blue) windows. Filled boxes on the MRD show ADC hits in the beam-on timing window.

zontal direction perpendicular to the beam direction. The origin is located on the most upstream surface of SciBar in the $z$ dimension, and at the center of the SciBar scintillator plane in the $x$ and $y$ dimensions. Since each sub-detector is read out both vertically and horizontally, two views are defined; the top view $(z-x$ projection $)$ and the side view $(z-y$ projection).

\subsubsection{Detector alignment}

For SciBar and the EC, the alignment of the detectors was performed with respect to the dark box. The position of each SciBar scintillator layer was surveyed during installation to the dark box. After installing the detector to the detector hall, the position of each layer was measured again using cosmic-ray muons; the $x$ and $y$ positions of each layer are determined so that the $\chi^{2}$, calculated from positions of hits in the layer and the fitted line using hits in the other layers, is minimized. Figure 5.18 shows the displacement of each SciBar scintillator layer with respect to the most upstream layer, measured with the two different methods. As seen in the figure, the results are fairly consistent with each other, and the precision of the measurements is estimated to be $1 \mathrm{~mm}$. The relative positions of the EC planes with respect to SciBar were determined using cosmic-ray muons. The precision of the relative positions is estimated to be a few $\mathrm{mm}$.

The positions of the dark box and each MRD scintillator layer were surveyed with 

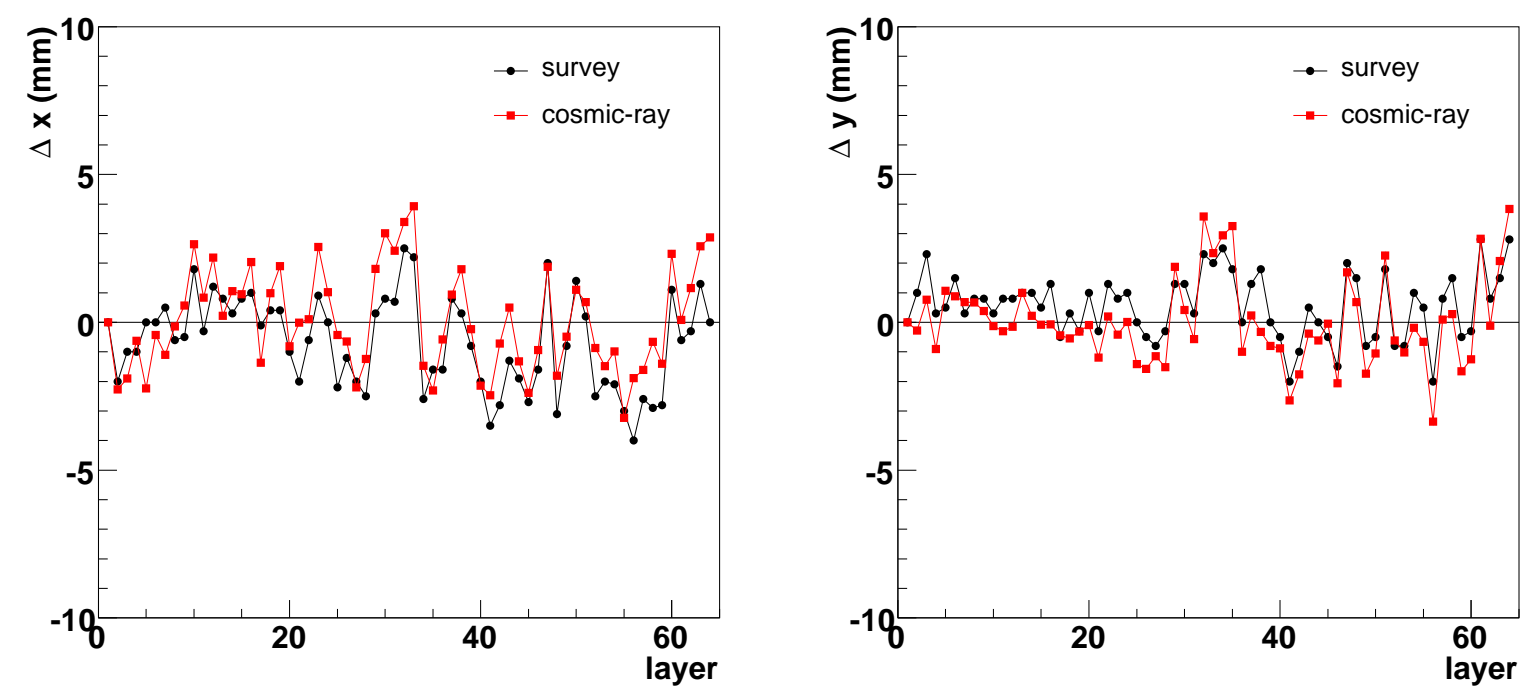

Figure 5.18: Displacement of each SciBar scintillator layer with respect to the most upstream layer.

respect to the detector hall. The measurement allows us to determine the MRD position in terms of the detector coordinates. The position of the detector hall with respect to the beamline was also surveyed. The measurement results are reflected in the MC simulation and event reconstruction. According to the survey data, the distance between the center of the beryllium target and the center of the SciBar detector is $99.92 \mathrm{~m}$, with the SciBooNE detector located on beam axis within a tolerance of a few $\mathrm{cm}$.

\subsection{Detector simulation}

The GEANT4 framework is used for the detector simulation. The detector simulation includes a detailed geometric model of the detector, including the detector frame and experimental hall and soil, which is based on survey measurements taken during detector construction.

\subsubsection{Simulation of detector responses}

In the detector simulation of SciBar, low level data parameters are used as input to the simulation whenever possible. The energy loss of a charged particle in a single strip is simulated by GEANT, and this energy scale is tuned using cosmic-ray data. Scintillator quenching is simulated using Birk's law with a measured value of Birk's constant. The energy deposited by a charged particle is converted to photoelectrons using conversion factors measured for each channel with cosmic muons. The measured light attenuation length of each fiber is used in the simulation. Crosstalk between nearby MA-PMT channels is simulated using measured values. The number of photoelectrons is smeared by Poisson statistics, and the single photoelectron resolution of the MA-PMT is simulated. To simulate the digitization of the PMT signal, the number of photoelectrons is converted to ADC counts, and then electronics noise and threshold effects of the TA are simulated. 
TDC hit simulation includes light propagation delays in the WLS fibers. A logical OR of 32 MA-PMT channels is made for each TDC channel, and the time of each hit is converted to TDC counts. Multiple TDC hits in each channel are simulated.

In the EC detector simulation, true energy deposition in scintillating fibers in the detector is converted to the number of photoelectrons using a conversion factor which is measured for each channel with cosmic-ray muons. The attenuation of light in the fiber is simulated using the measured attenuation length value. The number of photoelectrons is smeared by Poisson statistics and by the PMT resolution, and then converted to ADC counts. The time-dependent ADC gain due to the overshoot of the PMT signal is simulated based on a measurement with cosmic muons. Electronics noise is also simulated.

For the detector simulation of the MRD, true energy deposition in each scintillator is converted to ADC counts using the conversion factor measured with cosmic muons. The attenuation of light in the scintillator as well as electronics noise are simulated. Gaps between scintillator counters in each plane, which cause inefficiency, are included in the simulation. The time of energy deposition is digitized and converted into TDC counts.

\subsubsection{Simulation of pion interaction in detector}

The Bertini cascade model within GEANT4 [99] is used to simulate the interactions of hadronic particles with detector materials. Among all the hadronic interactions, the interactions of pions are most important to these analyses. To check the validity of the simulation in our interested energy region $\left(T_{\pi}<500 \mathrm{MeV}\right)$, the simulated cross section of pion-carbon interaction is compared with external measurements [85,100-104]. Figure 5.19 shows the $\pi^{+}$-carbon interaction cross sections as a function of pion kinetic energy. The cross sections for pion absorption, pion charge exchange, total inelastic interaction (absorption, charge exchange, and inelastic scattering), and total interaction are shown separately. The MC simulation reproduces the data. ${ }^{2}$ A $10 \%$ difference of the total cross section between the MC simulation and data is seen for higher energy pion, and therefore it is considered when we evaluate systematic errors.

\footnotetext{
${ }^{2}$ The default GEANT4 version 4.9.1 simulates less pion absorption. For these analyses, the cross section in GEANT4 is scaled to reproduce external measurements.
} 


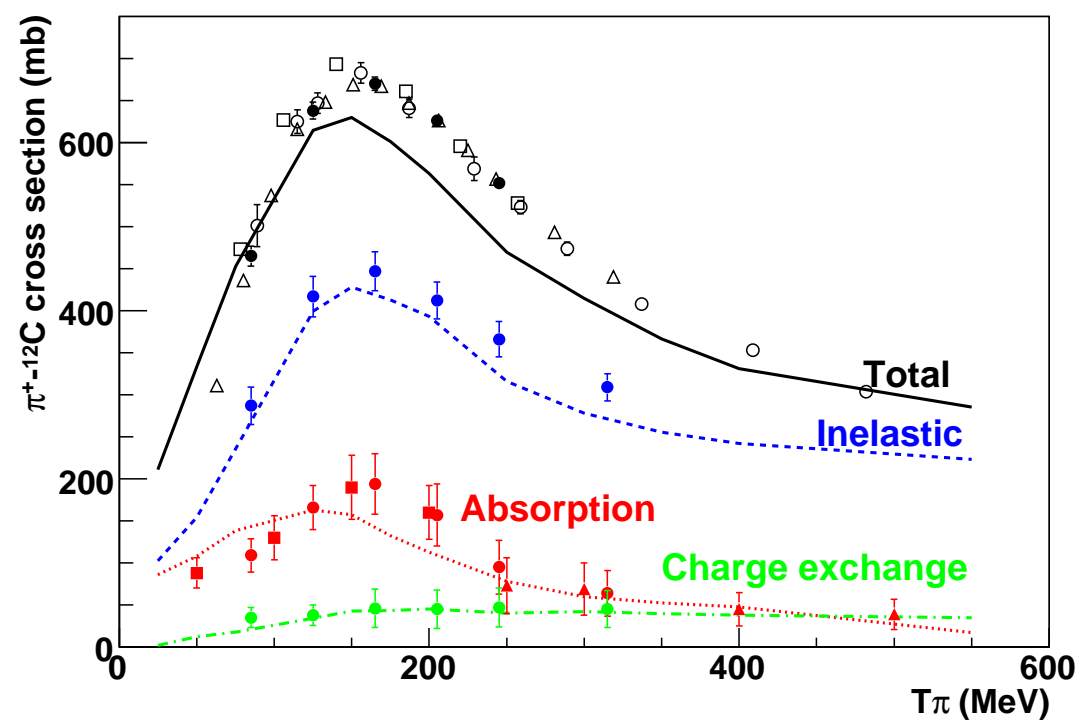

Figure 5.19: $\pi^{+}$-carbon interaction cross sections as a function of pion kinetic energy. The data points are taken from the following experiments: (•)Ashery [85], ( $\square$ )Wilkin [100], $(\bigcirc)$ Clough [101], $(\triangle)$ Carroll [102], (ם)Ransome [103], and (ム)Jones [104]. 


\section{Chapter 6}

\section{Data Summary}

The SciBooNE experiment took data from June 2007 until August 2008. The datataking is divided into three periods depending on the polarity of the horn: Run-1 (Antineutrino mode), Run-2 (Neutrino mode), and Run-3 (Antineutrino mode). In this chapter, we describe data quality cuts which are applied before any physics analyses, and then we summarize data set used in these analyses. The stability of the beam data-taking is also shown.

\subsection{Data quality cuts}

Only spills that satisfy certain data quality cuts are used for physics analyses. The purpose of the data quality cuts is to ensure that the experimental apparatus is functioning properly. This includes both the beam and detector.

\subsubsection{Beam}

Table 6.1 summarizes beam quality cuts and fractions of the total number of protons on target that fail each cut. Each cut is described below. Distributions of the beam quality variables for a typical beam condition are shown in Figure 6.1. Overall, beam quality cuts reject less than $1 \%$ of the total number of protons on target accumulated during the run.

Table 6.1: Beam quality cuts and fractions of the total number of protons on target that fail each cut.

\begin{tabular}{llr}
\hline \hline Cut & & Fail fraction \\
\hline Proton beam intensity & TOR875 $>0.1 \times 10^{12}$ p.p.p & $0.06 \%$ \\
Toroid agreement & $2 \times \frac{\mid \text { TOR875-TOR860 }}{(\text { TOR875+TOR860) }}<10 \%$ & $0.07 \%$ \\
Peak horn current & $\left|I_{\text {peak }}\right|>170 \mathrm{kA}$ & $0.09 \%$ \\
Targeting efficiency & $\epsilon_{\text {target }}>95 \%$ & $0.15 \%$ \\
GPS time difference & $\left|t_{\text {beam }}-t_{\text {det }}\right|<10 \mathrm{msec}$ & $0.13 \%$ \\
\hline \hline
\end{tabular}

1) protons per pulse 


\section{Proton beam intensity}

The intensity of the primary proton beam is measured on a spill-by-spill basis using two toroidal current transformer (toroids), TOR860 and TOR875, located $200 \mathrm{~m}$ and $5 \mathrm{~m}$ upstream from the target along the beamline, respectively. The number of protons hitting on the beryllium target is measured using TOR 875 . Since the proton beam typically contains $4-5 \times 10^{12}$ protons per pulse, beam spills which contain at least $0.1 \times 10^{12}$ protons per pulse are selected (Figure 6.1 (a)).

\section{Toroid agreement}

In order to ensure that the toroids are functioning and that the proton beam is transported properly, the agreement between two toroid readouts is required to be better than $10 \%$ (Figure $6.1(\mathrm{~b})$ ).

\section{Peak horn current}

The peak horn current is also measured on a spill-by-spill basis. The actual operating values are $174 \mathrm{kA}$ for neutrino mode and $-176 \mathrm{kA}$ in antineutrino mode. The absolute peak horn current, $\left|I_{\text {peak }}\right|$, is required to be greater than $170 \mathrm{kA}$ (Figure 6.1 (c)).

\section{Targeting efficiency}

The targeting efficiency, a fraction of the beam passes through the entire length of the target, is estimated using measurements with two sets of the horizontal and vertical beam position monitors, located upstream of the target. The efficiency is required to be greater than 95\% (Figure $6.1(\mathrm{~d})$ ).

\section{GPS time difference}

Since the BNB receives one train of proton beam pulses in a row at $15 \mathrm{~Hz}$, the minimum time span between beam spills is $67 \mathrm{msec}$. To ensure that the beam and detector information is correctly merged, the difference between the beam and detector GPS time stamps, $\left|t_{\text {beam }}-t_{\text {det }}\right|$, is required to be within 10 msec (Figure $6.1(\mathrm{e})$ ).

\subsubsection{Detector}

Approximately 1-2\% of beam spills were lost due to the dead time of the DAQ system during the run switch done manually every $\sim 8$ hours, and due to the initialization failure of SciBar electronics which sometimes happened right after the run switch. In addition, there were occasionally detector down time due to maintenance works. Approximately $2 \%$ of beam spills were lost during the detector maintenance.

Pedestals, the supplied high-voltages, and responses to cosmic-ray muons of each subdetector, and the response of SciBar to the LED light, are monitored continuously, and only the periods where all the sub-detectors are functioning are selected. Less than $1 \%$ of protons on target are rejected because of these cuts. 


\section{Run011640 [ Sun Jan 20 12:14:00 2008 - Sun Jan 20 18:08:52 2008 ]}
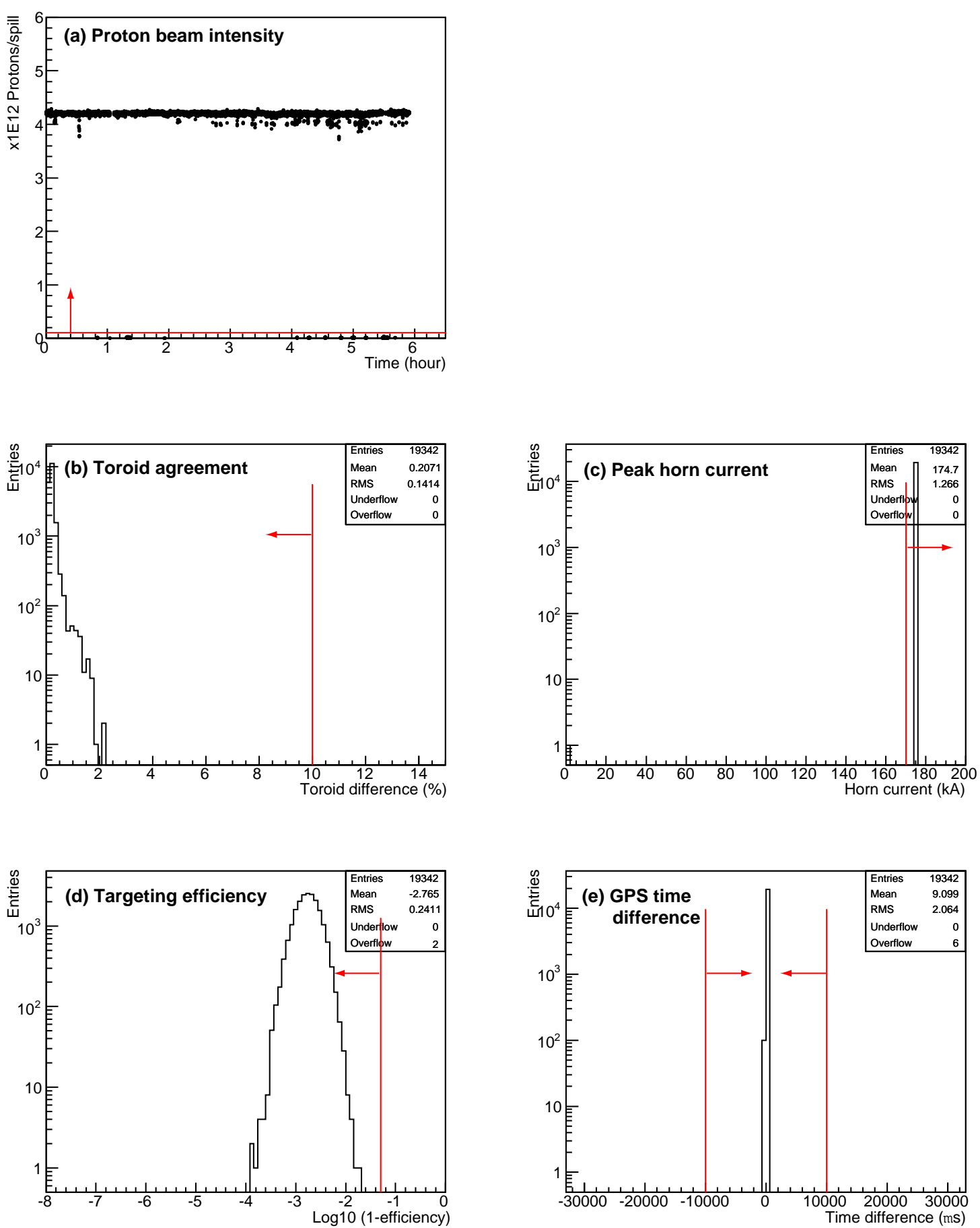

Figure 6.1: Distributions of the beam quality variables for a typical beam condition. 


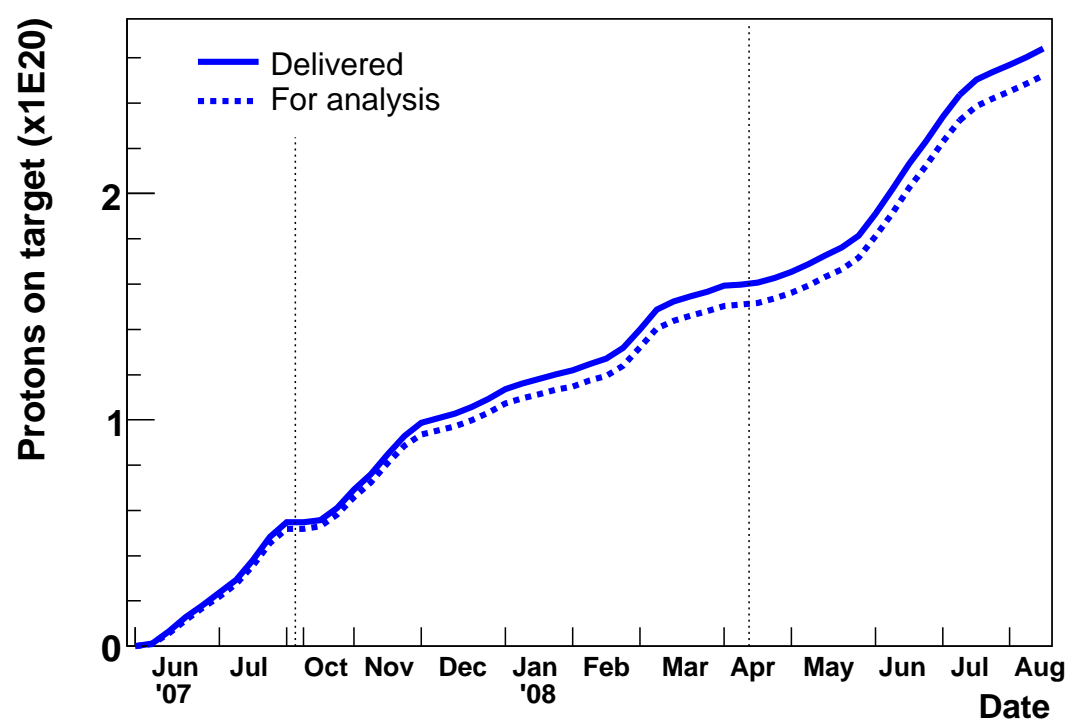

Figure 6.2: History of the accumulated number of protons on target (POT).

Table 6.2: Summary of SciBooNE data-taking. The table shows the number of protons on target (POT) collected after application of data quality cuts, as described in the text.

\begin{tabular}{lll}
\hline \hline Run & Period & POT \\
\hline Run 1 (Antineutrino) & Jun. 2007 - Aug. 2007 & $0.52 \times 10^{20}$ \\
Run 2 (Neutrino) & Oct. 2007 - Apr. 2008 & $0.99 \times 10^{20}$ \\
Run 3 (Antineutrino) & Apr. 2008 - Aug. 2008 & $1.01 \times 10^{20}$ \\
\hline \hline
\end{tabular}

\subsection{Summary of data-taking}

\subsubsection{Number of protons on target}

Fig. 6.2 shows a history of the accumulated number of protons on target; the two curves show the total protons on target for all events and the protons on target for events passing all data quality cuts, described above. In total, $2.64 \times 10^{20}$ protons on target were delivered to the beryllium target during the SciBooNE data run. After all beam and detector quality cuts, $2.52 \times 10^{20}$ protons on target are usable for physics analyses. The data collection efficiency is $95.5 \%$. The number of protons on target for each run period is summarized in Table 6.2.

In these analyses, the full neutrino data sample is used, corresponding to $0.99 \times$ $10^{20}$ protons on target satisfying all data quality cuts, collected between October 2007 and April 2008. The antineutrino data sample collected after the neutrino data-taking period, corresponding to $1.01 \times 10^{20}$ protons on target, is used in the analysis presented in Chapter 8. The antineutrino data sample collected before the neutrino data-taking period is not considered in these analyses. 


\subsubsection{Neutrino event rate stability}

In order to confirm the stability of the beam data-taking, the event rate of charged current candidates which occur in SciBar is monitored at the semi-online level, using simple selections:

- If there are more than three hits in each view in an event, with a hit threshold of two photoelectrons, the event is selected, and a simple tracking is applied.

- The tracking algorithm searches for contiguous hits in each view to reconstruct a two dimensional track projection. Three dimensional tracks are reconstructed by matching the $z$-edges of two dimensional tracks. Events with at least one track are selected.

- The longest track is required to penetrate more than four layers of SciBar to reject neutral current events. Such a track is considered as a muon candidate.

- The neutrino interaction vertex is defined as the most upstream edge of the muon candidate. We select events whose vertices are in the SciBar fiducial volume, defined to be $\pm 130 \mathrm{~cm}$ in both the $x$ and $y$ dimensions, and $2.62 \mathrm{~cm}<z<157.2 \mathrm{~cm}$.

- The muon candidate is required to reach the most downstream layer of SciBar in order to reject cosmic-ray muons coming in from the side edge of the detector.

- Events in a $2 \mu$ sec beam timing window are selected. The contamination of cosmicray background in the beam timing window can be estimated using a beam-off timing window.

This reconstruction is used only for operations related studies, and not for the analyses described in this thesis.

Figure 6.3 shows the stability of the number of charged current candidate events in SciBar normalized to the number of protons on target. The BNB receives $(2-6) \times 10^{16} \mathrm{POT} / \mathrm{hr}$ depending on accelerator cycle, and therefore the vertical axis corresponds approximately to the number of events per hour in a typical beam condition. The contamination of cosmic-ray background is estimated to be $1 \%$ using a beam-off timing window, and subtracted in the figure. The event rate was stable during each of neutrino and antineutrino mode runnings. The event rate difference between neutrino and antineutrino modes can be seen clearly. This is due to the difference between positively- and negatively-charged meson production cross sections in the proton-beryllium interaction and the difference between neutrino- and antineutrino-nucleus cross sections.

A similar event rate monitoring by using the MRD was also performed independently. Figure 6.4 shows the stability of the number of charged current candidate events in the MRD normalized to the number of protons on target. The event rate was stable during each of neutrino and antineutrino mode runnings. The event rate difference between SciBar and the MRD is due to the difference in the fiducial mass and the selection efficiency.

In summary, we confirmed that both the neutrino beam and detector had been stable during the whole data-taking period. 


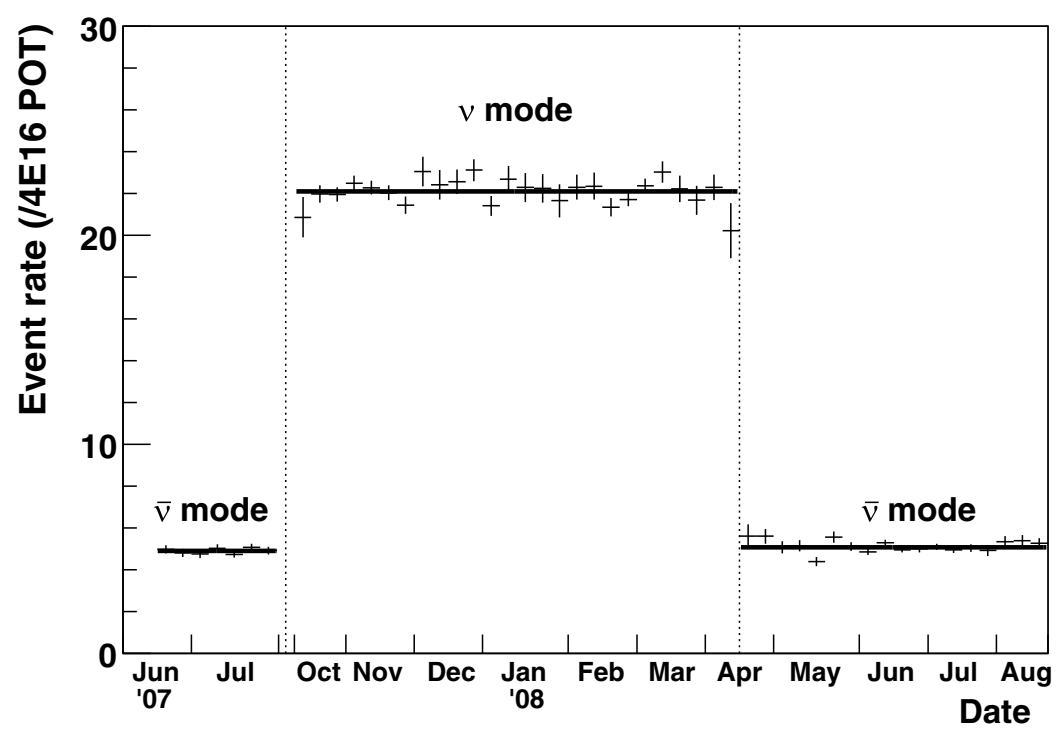

Figure 6.3: Stability of the number of charged current candidate events in SciBar normalized to the number of protons on target.

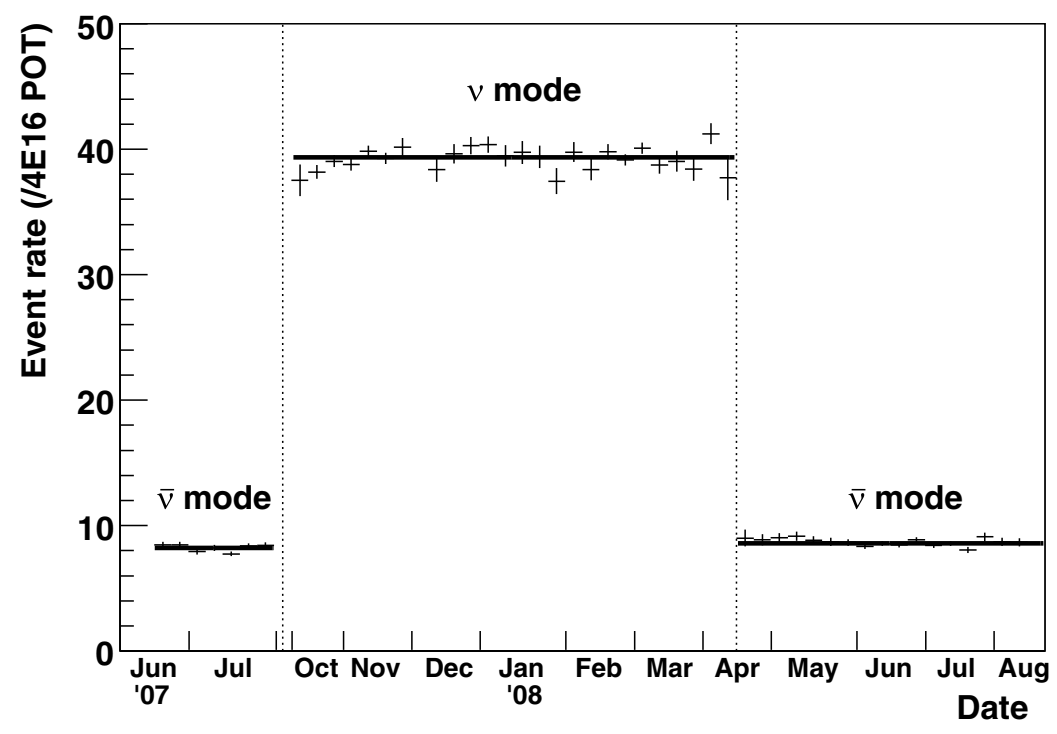

Figure 6.4: Stability of the number of charged current candidate events in the MRD normalized to the number of protons on target. 


\section{Chapter 7}

\section{Study of Charged Current Coherent Pion Production}

\subsection{Analysis overview}

The experimental signature of charged current coherent pion production is the existence of two and only two tracks originating from a common vertex, both consistent with minimum ionizing particles (a muon and a charged pion), with a small momentum transfer to the nucleus. According to the MC simulation, the dominant background for this process is charged current resonant charged pion production, $\nu N \rightarrow \mu^{-} N \pi^{+}$, where the proton or neutron is not detected. In order to suppress resonant pion backgrounds,

- Protons with momentum below the tracking threshold are detected by their large energy deposition around the vertex.

- Events in which a pion-like track goes forward are selected.

Two charged current coherent pion samples are made, events with a muon stopping in the MRD and events with a muon penetrating the MRD, and this enables us to measure the charged current coherent pion production at different neutrino energies.

The amount of background in the signal region is estimated with the MC simulation. In order to constrain systematic uncertainties in the MC simulation, the MC distributions of reconstructed $Q^{2}$ (the square of the four-momentum transfer) outside the signal region are fitted to the data distributions.

We measure the cross section ratios to total charged current interaction, not absolute cross sections, in order to reduce the systematic uncertainty in the neutrino flux prediction. Furthermore, charged current inclusive samples for normalization are carefully chosen to have similar mean neutrino energy to the corresponding charged current coherent pion samples.

\subsection{Charged current event selection}

The first step of the event reconstruction is to search for two-dimensional tracks in each view of SciBar using a cellular automaton algorithm [105]. For tracking, the hit threshold is set to two photoelectrons, corresponding to approximately $0.2 \mathrm{MeV}$. Three 
dimensional tracks are reconstructed by matching the timing and z-edges of the two dimensional tracks. The timing difference between two two dimensional tracks is required to be less than $50 \mathrm{nsec}$, and the $z$-edge difference must be less than $6.6 \mathrm{~cm}$ for upstream and downstream edges. Reconstructed tracks are required to have at least three-layer penetration, and therefore the minimum length of a reconstructed track is $8 \mathrm{~cm}$ in the beam direction. According to the MC simulation, $96 \%$ of charged current interactions in SciBar are reconstructed to have at least one track.

To identify charged current events, we look for events in which at least one reconstructed track in SciBar is matched with a track or hits in the MRD. Such a track is defined as a SciBar-MRD matched track. The most energetic SciBar-MRD matched track in any event is considered as a muon candidate. For matching a MRD track to a SciBar track, the upstream edge of the MRD track is required to be on either one of the first two layers of the MRD. The transverse distance between the two tracks at the first layer of the MRD must be less than $30 \mathrm{~cm}$. The requirement on the difference between track angles with respect to the beam direction is given by $\left|\theta_{\mathrm{MRD}}-\theta_{\mathrm{SB}}\right|<\theta_{\max }$, where $\theta_{\max }$ is a function of the length of the MRD track, varying between 0.4 radian and 1.1 radians. For track reconstruction in the MRD, at least two hit layers in each view are needed, and thus this matching method is used for tracks which penetrate at least three steel plates. If no MRD track is found, we extrapolate the SciBar track to the MRD and search for nearby contiguous hits in the MRD identifying a short muon track. For matching MRD hits to a SciBar track, the MRD hit is required to be within a cone with an aperture of \pm 0.5 radian and a transverse offset within $10 \mathrm{~cm}$ of the extrapolated SciBar track at the upstream edge of the MRD. The timing difference between the SciBar track and the track or hits in the MRD is required to be within 100 nsec. The matching criteria impose a muon momentum threshold of $350 \mathrm{MeV} / c$. Detailed descriptions of track reconstruction are given in Appendix A.

Then, we apply the 1st layer veto cut, the fiducial volume cut and the event timing cut to reduce backgrounds due to neutrino interactions which occurred outside the detector and cosmic-ray muons as described below.

\subsubsection{1st layer veto}

Particles produced by neutrino interactions in the upstream wall or soil often come into SciBar. Therefore, we reject events in which hits adjacent to the muon candidate exist on the most upstream layer of SciBar. The hit threshold for this veto cut is set to two photoelectrons.

\subsubsection{Fiducial volume cut}

The neutrino interaction vertex is reconstructed as the upstream edge of the muon track. Figure 7.1 shows the difference between the reconstructed and true vertex estimated with the MC simulation. The vertex resolution is approximately $0.5 \mathrm{~cm}$ in each dimension, estimated by fitting with a Gaussian. Since the $z$ position is discretely determined and the true vertex is uniformly distributed, the resolution in the $z$ dimension is expected to be $1.3 / \sqrt{12} \sim 0.4 \mathrm{~cm}$. Therefore, the $z$ vertex resolution obtained from Figure 7.1 is consistent with the expectation. If there is a backward-going track emerging from the same point as the SciBar-MRD matched track, the backward-going track is often 

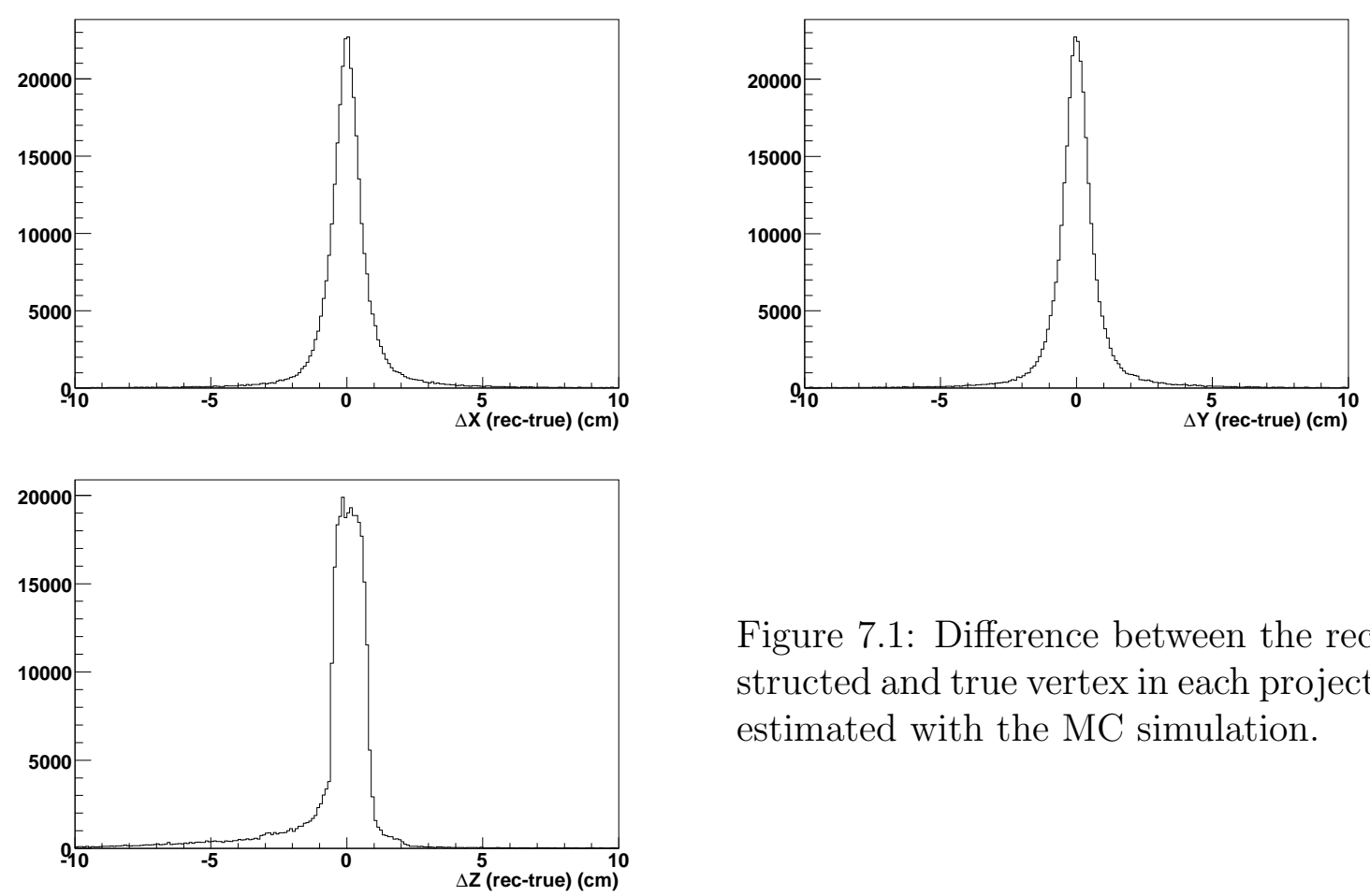

Figure 7.1: Difference between the reconstructed and true vertex in each projection estimated with the MC simulation.

reconstructed as a part of the SciBar-MRD matched track, and thus the reconstructed vertex is shifted to upstream. This causes a tail in the negative $\Delta z$ in Figure 7.1.

We select events whose neutrino interaction vertices are in the SciBar fiducial volume. The fiducial volume is defined as

$$
\left\{\begin{array}{l}
-130 \mathrm{~cm}<x<130 \mathrm{~cm}, \\
-130 \mathrm{~cm}<y<130 \mathrm{~cm}, \\
2.62 \mathrm{~cm}<z<157.2 \mathrm{~cm} \text { (2nd-60th layer) }
\end{array}\right.
$$

The total mass in the fiducial volume is

$$
260 \mathrm{~cm} \times 260 \mathrm{~cm} \times 2.6 \mathrm{~cm} \times 59 \text { layers } \times 1.021 \mathrm{~g} / \mathrm{cm}^{3}=10.6 \text { tons } .
$$

Figure 7.2 shows the reconstructed vertex distribution of events in the $2 \mu$ sec beam timing window after 1st layer veto cut. To demonstrate how each fiducial cut works, the distributions of each projection with all the other cuts applied are shown. The data excess outside of the fiducial volume is due to cosmic-ray muons and incoming particles produced by neutrino interactions in surrounding materials, which is not simulated in the MC simulation. The background contamination due to neutrino events which occur in the EC and MRD after the fiducial volume cut is estimated with the MC simulation to be $2.0 \%$ and $0.5 \%$, respectively.

\subsubsection{Event timing cut}

The event timing is defined as the mean time of the reconstructed muon track. Figure 7.3 shows the event timing distribution after the fiducial volume cut. Finally, we 

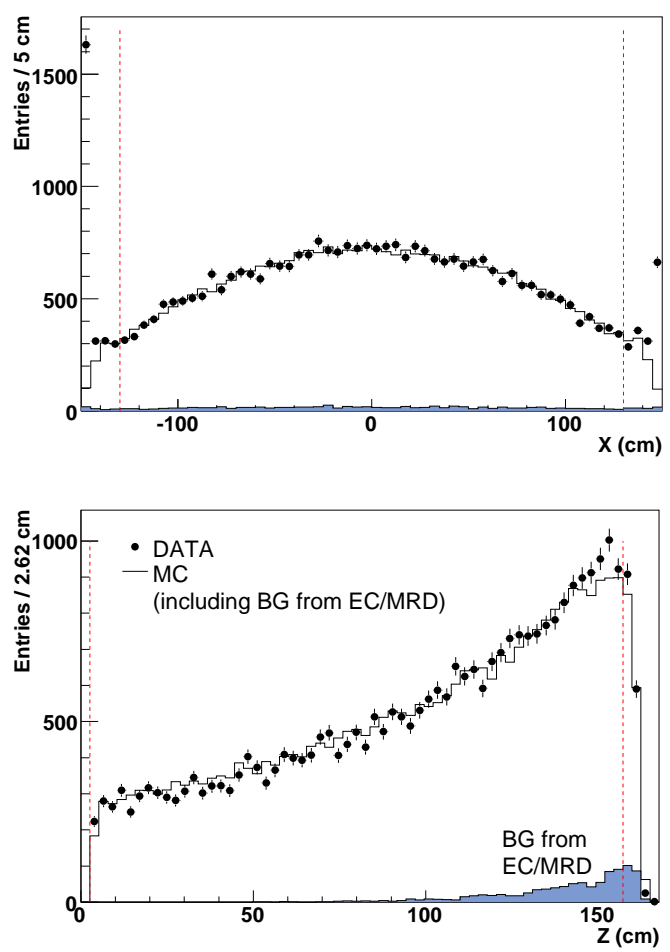

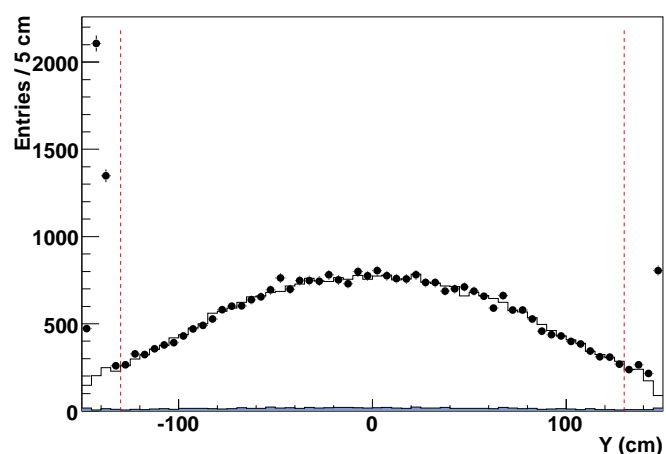

Figure 7.2: Reconstructed vertex distribution of beam-timing events after 1st layer veto cut. Each projection shows the distribution with all cuts applied except for the fiducial volume cut in the projection. Dashed lines indicate the fiducial volume cut. The MC distribution is normalized with the SciBar-MRD matched sample after all cuts.

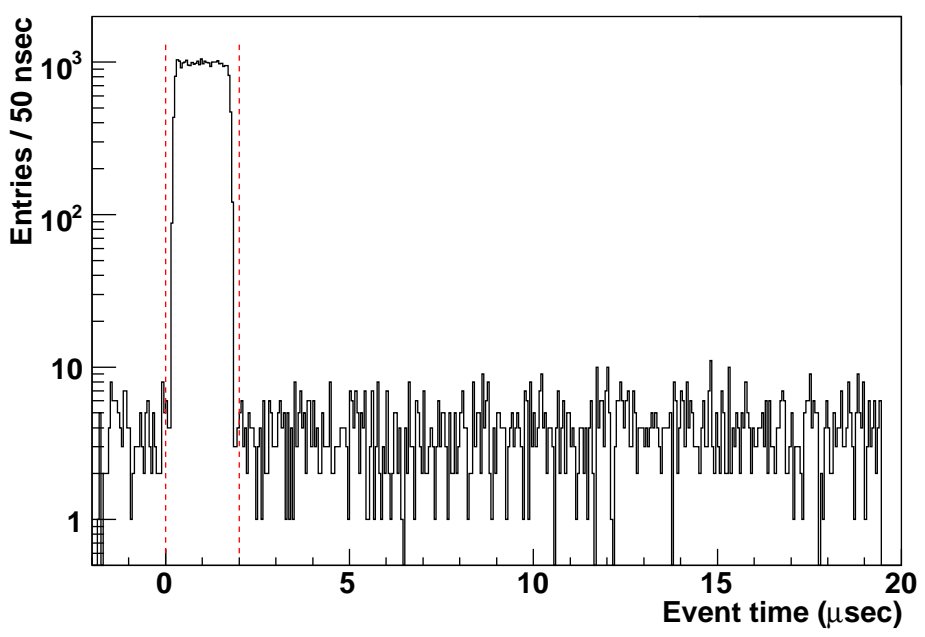

Figure 7.3: Event timing distribution of the SciBar-MRD matched events. Dashed lines indicate the $2 \mu$ sec beam timing window.

select events in the $2 \mu$ sec beam timing window $(0 \mu$ sec $<t<2 \mu$ sec $)$. The cosmic-ray background contamination in the beam timing window is only $0.5 \%$, estimated using a beam-off timing window $(5 \mu \mathrm{sec}<t<15 \mu \mathrm{sec})$. 

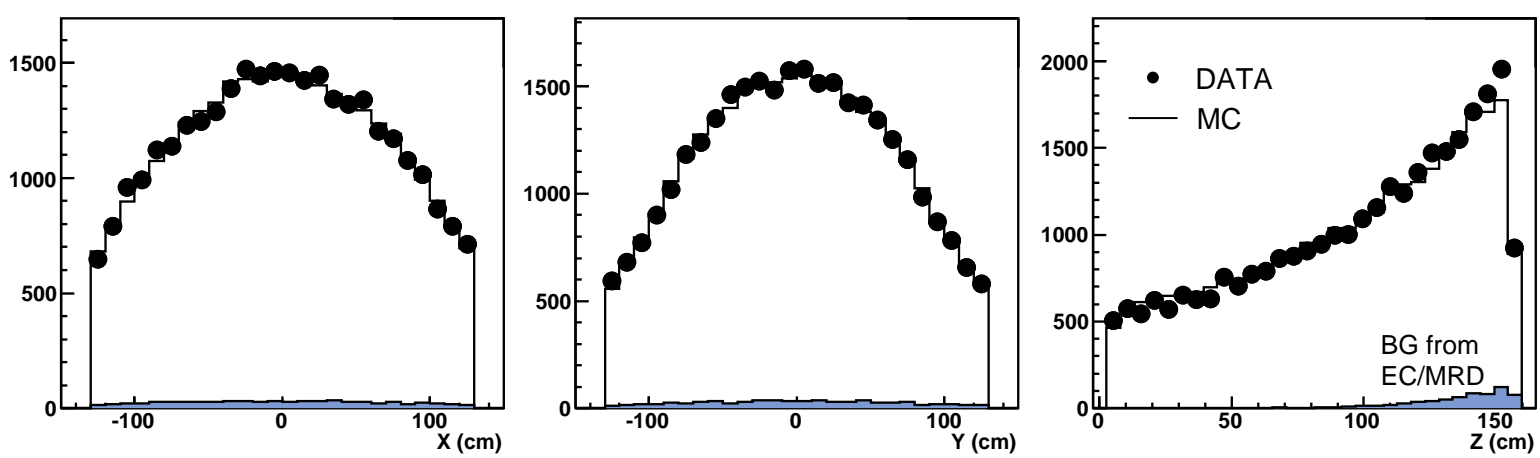

Figure 7.4: Reconstructed vertex distribution of the SciBar-MRD matched sample.

\subsubsection{Summary of the SciBar-MRD matched sample}

We select 30,337 SciBar-MRD matched events in data. Figure 7.4 shows the reconstructed vertex distribution of the sample for data and the MC simulation. The detection efficiency and purity of $\nu_{\mu}$ charged current events in the sample are estimated with the MC simulation as follows.

$$
\begin{aligned}
\nu_{\mu} \text { CC efficiency } & \equiv \frac{\text { the number of } \nu_{\mu} \text { CC events passed the selection }}{\text { the number of } \nu_{\mu} \text { CC events generated in the FV }} \\
& =0.279 \\
\nu_{\mu} \text { CC purity } & \equiv \frac{\text { the number of selected } \nu_{\mu} \text { CC events }}{\text { the number of events passed the selection }} \\
& =0.928 .
\end{aligned}
$$

Impurity comes from $\nu_{\mu}$ neutral current events $(3.0 \%), \bar{\nu}_{\mu}$ charged current events $(1.6 \%)$, and neutrino events which occur in the EC/MRD (2.5\%).

Figure 7.5 shows $\nu_{\mu}$ charged current efficiencies versus true muon momentum $\left(p_{\mu}\right)$ and angle $\left(\theta_{\mu}\right)$ for the SciBar-MRD matched sample. Filled histograms in the efficiency plots indicate events in which a particle other than muon, mainly proton or charged pion, is reconstructed as the muon candidate. Due to the MRD matching requirement, there is no efficiency for the events with a backward-going muon. Figure 7.6 shows $\nu_{\mu}$ charged current efficiencies versus true neutrino energy $\left(E_{\nu}\right)$ and the square of four-momentum transfer $\left(Q^{2}\right)$ for the SciBar-MRD matched sample. The average neutrino beam energy for true charged current events in the sample is $1.2 \mathrm{GeV}$.

Figure 7.7 shows the number of steel planes penetrated by the muon candidate for the SciBar-MRD matched sample.

This SciBar-MRD matched sample is our standard charged current data set and defines the MC normalization; i.e. the MC distributions are normalized to the number of SciBarMRD matched events in data.

\subsubsection{MRD stopped and MRD penetrated samples}

The SciBar-MRD matched sample is further divided into sub-samples based on the end point of the muon candidate as shown in Figure 7.8. If the muon candidate stops in 

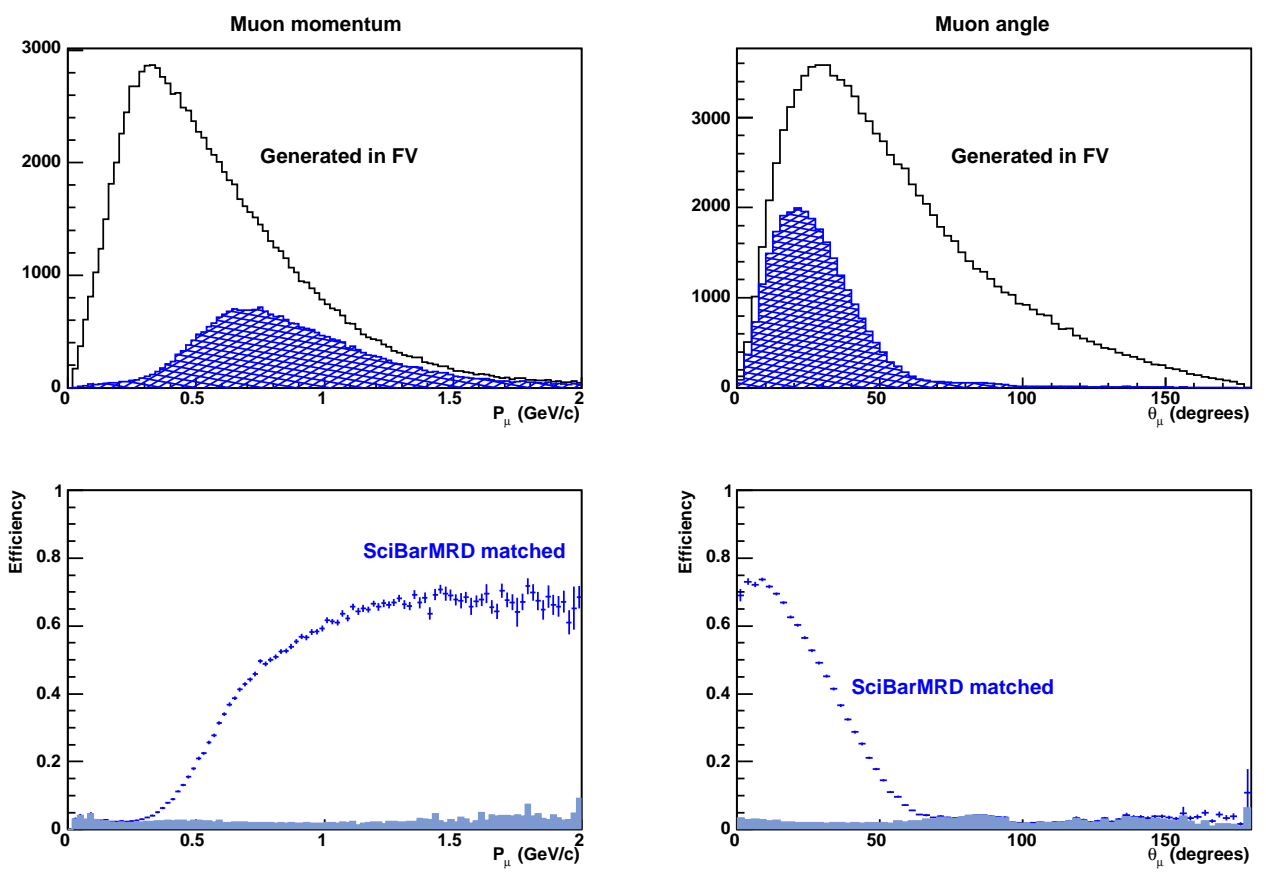

Figure 7.5: $\nu_{\mu}$ CC efficiencies versus true muon momentum (left) and angle (right) for the SciBar-MRD matched sample. Filled histograms indicate events in which a particle other than muon, mainly proton or charged pion, is reconstructed as the muon candidate.
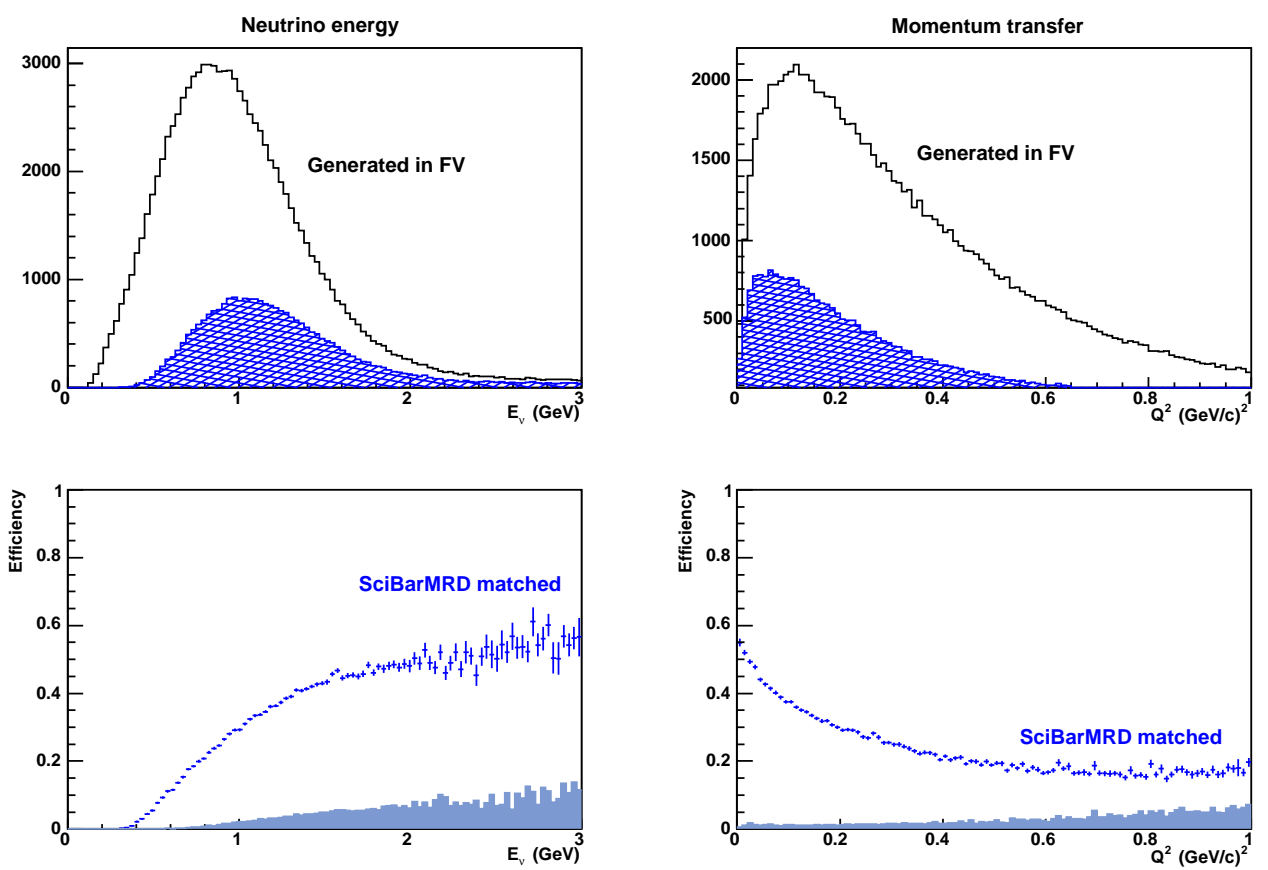

Figure 7.6: $\nu_{\mu}$ CC efficiencies versus true neutrino energy (left) and the square of fourmomentum transfer (right) for the SciBar-MRD matched sample. Filled histograms indicate events in which a particle other than muon, mainly proton or charged pion, is reconstructed as the muon candidate. 


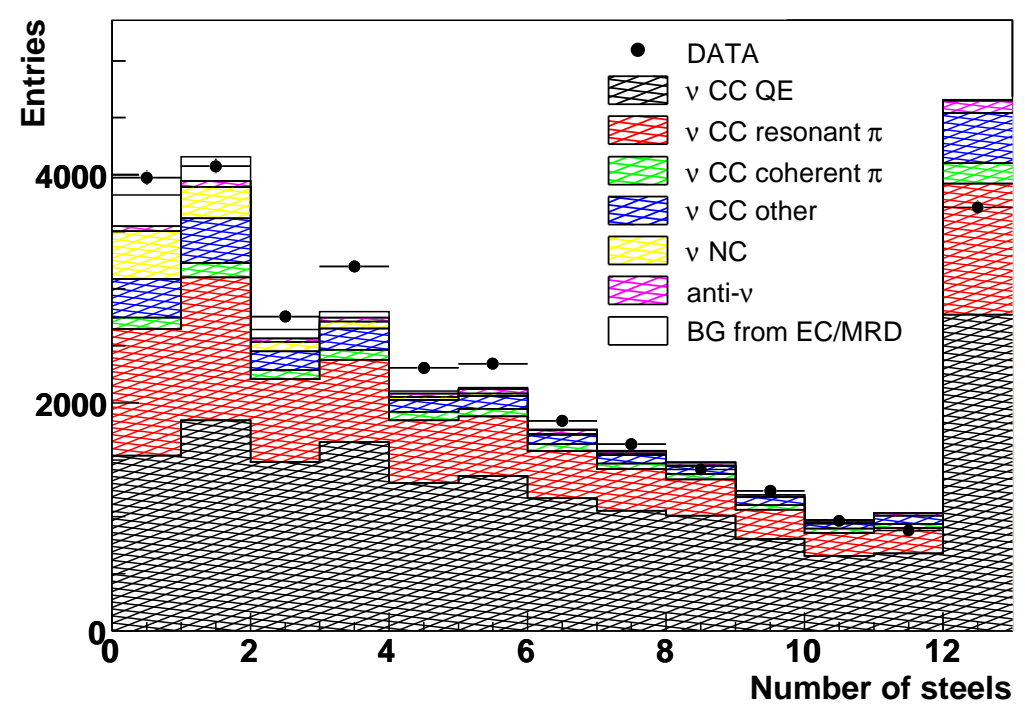

Figure 7.7: Number of steel planes penetrated by the muon track for the SciBar-MRD matched sample

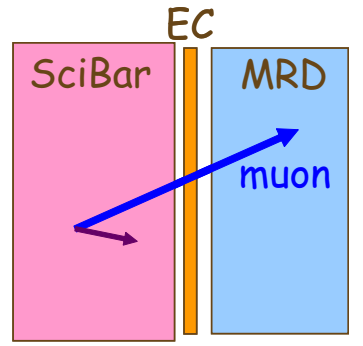

(a) MRD stopped

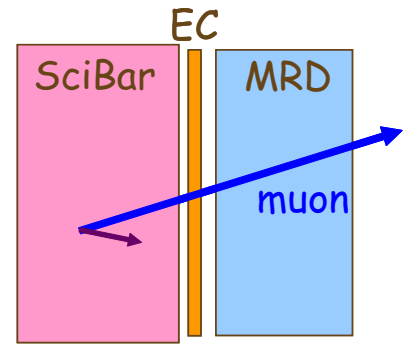

(b) MRD penetrated

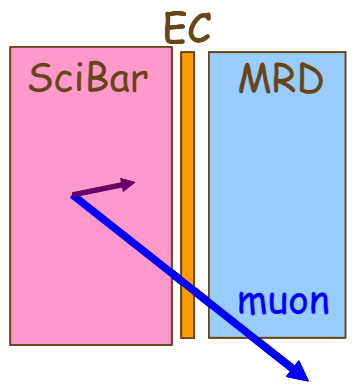

(c) MRD side-escaped

Figure 7.8: Schematic drawings of the MRD stopped, MRD penetrated, and MRD sideescaped events.

the MRD, namely the end point of the muon candidate is neither at the most downstream plane of MRD nor at the side edge channel of the MRD, the event is classified into the "MRD stopped" sample. If the muon candidate goes through all the MRD planes, the event is categorized into the "MRD penetrated" sample. The events in which the muon candidate escapes from the side of the MRD are classified into the "MRD side-escaped" sample. Of these samples, we use the MRD stopped and MRD penetrated samples for the charged current coherent pion measurement.

Figure 7.9 shows $\nu_{\mu}$ charged current efficiencies versus true $p_{\mu}$ and $\theta_{\mu}$ for the MRD stopped and MRD penetrated samples. The MRD stopped sample has the efficiency for the muon momentum between $350 \mathrm{MeV} / c$ and $1.5 \mathrm{GeV} / c$, and the MRD penetrated sample requires muons with momentum above $1 \mathrm{GeV} / c$ and angle less than 30 degrees. Figure 7.10 shows $\nu_{\mu}$ charged current efficiencies versus true $E_{\nu}$ and $Q^{2}$ for the MRD stopped and MRD penetrated samples. The average neutrino beam energy for true charged current events in the MRD stopped and MRD penetrated samples are $1.0 \mathrm{GeV}$ and $2.0 \mathrm{GeV}$, 
respectively, enabling a cross section measurement at two different neutrino energy regions.

The 3D angle of the muon with respect to the beam direction is calculated using the slope of the track in each 2D projection in SciBar. The kinetic energy of the muon is calculated by the range and expected energy deposition per unit length $(d E / d x)$ in SciBar, EC and MRD,

$$
\begin{aligned}
E_{\mathrm{kin}} & =E^{\mathrm{SB}}+E^{\mathrm{EC}}+E^{\mathrm{MRD}} \\
& =\left.\frac{d E}{d x}\right|_{\mathrm{SB}} \times L_{\mathrm{SB}}+\frac{\Delta E_{0}^{\mathrm{EC}}}{\cos \theta_{\mu}}+E^{\mathrm{MRD}}\left(L_{\mathrm{MRD}}\right),
\end{aligned}
$$

where $E^{\mathrm{SB}}, E^{\mathrm{EC}}$, and $E^{\mathrm{MRD}}$ are the energy deposition in each detector. $L_{\mathrm{SB}}$ and $L_{\mathrm{MRD}}$ are the track length of the muon in SciBar and the range in the MRD, respectively. We set $d E /\left.d x\right|_{S B}$ to $2.04 \mathrm{MeV} / \mathrm{cm}$, and $\Delta E_{0}^{\mathrm{EC}}$, which is the energy deposited in the EC by a horizontally transversing minimum ionizing particle is set to $91 \mathrm{MeV}$, estimated with the GEANT4 simulation. $E^{\mathrm{MRD}}$ is calculated from a range to energy lookup table based on the MC simulation.

Figure 7.11 shows the difference between reconstructed and true muon momenta for the MRD stopped sample, estimated using the MC simulation. Figure 7.12 shows the difference between reconstructed and true muon angles for the MRD stopped sample. For muons stopping in the MRD, the average muon momentum and muon angular resolutions are $50 \mathrm{MeV} / c$ and 0.9 degree, respectively. The systematic uncertainty in the muon momentum scale is estimated to be $2 \%$ which is dominated by the difference among various calculations of the range to energy lookup table.

By assuming CC-QE kinematics, the reconstructed neutrino energy is calculated from the muon momentum $p_{\mu}$ and the angle $\theta_{\mu}$ as

$$
E_{\nu}^{r e c}=\frac{1}{2} \frac{\left(m_{p}^{2}-m_{\mu}^{2}\right)-\left(m_{n}-V\right)^{2}+2 E_{\mu}\left(m_{n}-V\right)}{\left(m_{n}-V\right)-E_{\mu}+p_{\mu} \cos \theta_{\mu}},
$$

where $m_{p}, m_{n}$ and $m_{\mu}$ are the mass of proton, neutron and muon, respectively. $V$ is the nuclear potential, which is set to $27 \mathrm{MeV}$. The reconstructed momentum transfer is calculated as

$$
Q_{\text {rec }}^{2}=2 E_{\nu}^{r e c}\left(E_{\mu}-p_{\mu} \cos \theta_{\mu}\right)-m_{\mu}^{2} .
$$

Figure 7.13 and Figure 7.14 show the expected resolutions of the reconstructed $E_{\nu}$ and $Q^{2}$ for the MRD stopped sample, respectively. CC-QE events are shown as a hatched histogram.

For muons exiting the MRD, only a lower limit on muon momentum is obtained, while the muon angle is determined with better resolution than that of stopping muon. Figure 7.15 shows the difference between the reconstructed and true muon momenta, and the difference between the reconstructed and true muon angles is shown in Figure 7.16. The reconstructed $E_{\nu}$ and $Q^{2}$ for the MRD penetrated sample are calculated from muon angle and partially-reconstructed muon energy, using Equation 7.6 and Equation 7.7, respectively. Figure 7.17 shows the difference between the reconstructed and true $E_{\nu}$ for the MRD penetrated sample, and Figure 7.18 shows the difference between the reconstructed and true $Q^{2}$ for the MRD penetrated sample. Although only a part of the muon energy is observed, $Q^{2}$ is relatively well reconstructed because of the small muon angle. 

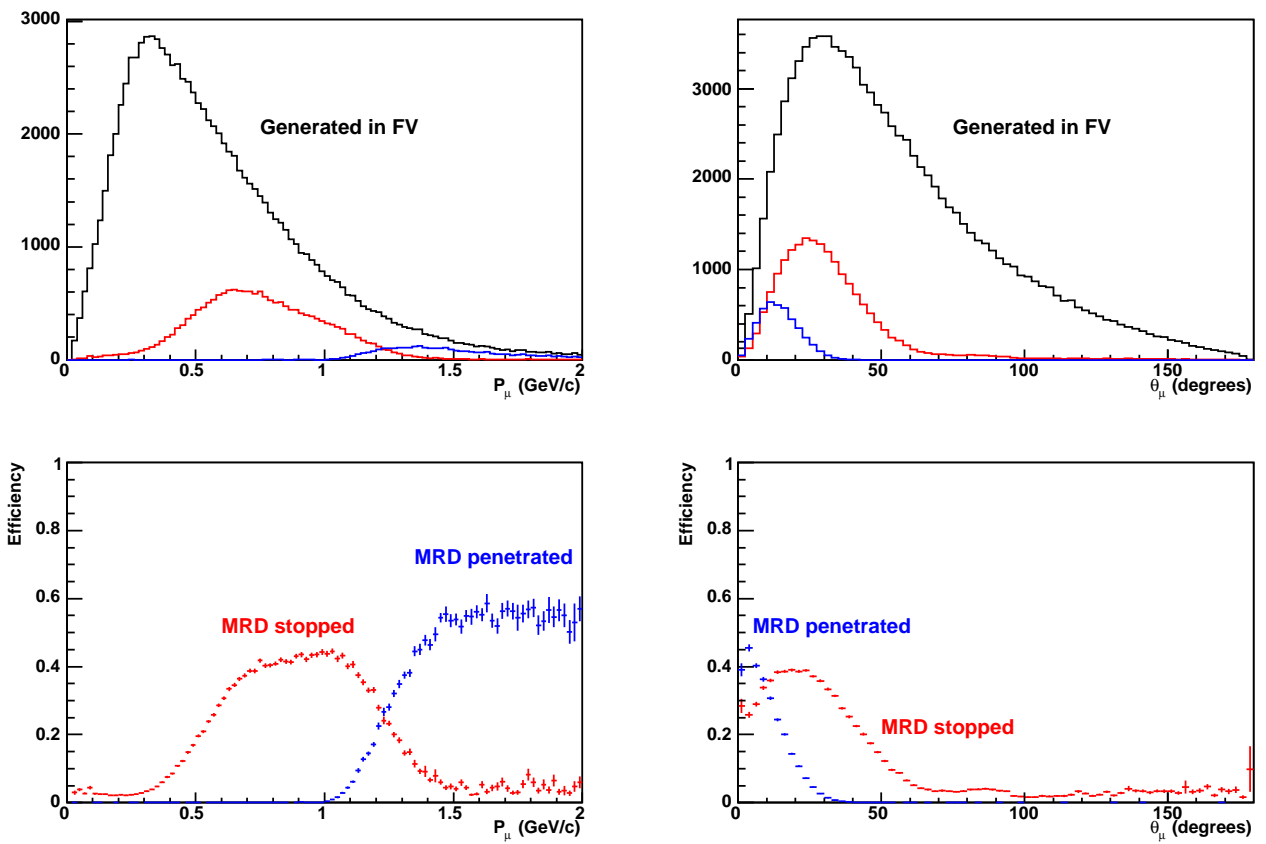

Figure 7.9: $\nu_{\mu}$ CC efficiencies versus true muon momentum (left) and angle (right) for the MRD stopped and MRD penetrated samples.
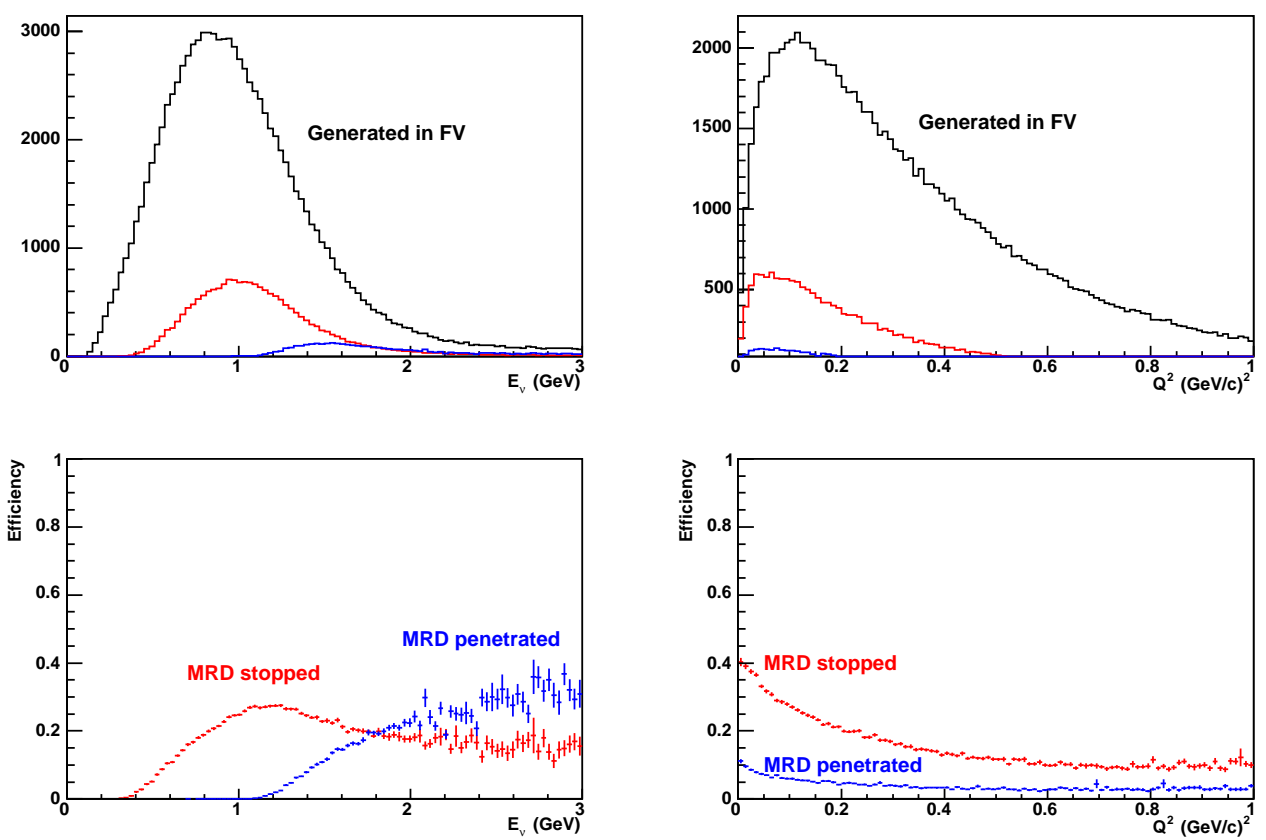

Figure 7.10: $\nu_{\mu}$ CC efficiencies versus true neutrino energy (left) and the square of fourmomentum transfer (right) for the MRD stopped and MRD penetrated samples. 


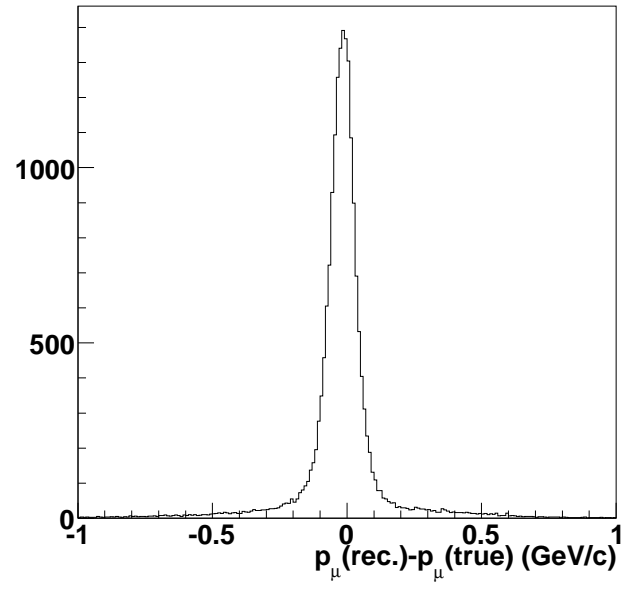

Figure 7.11: Difference between the reconstructed and true muon momenta for the MRD stopped sample estimated with the MC simulation.

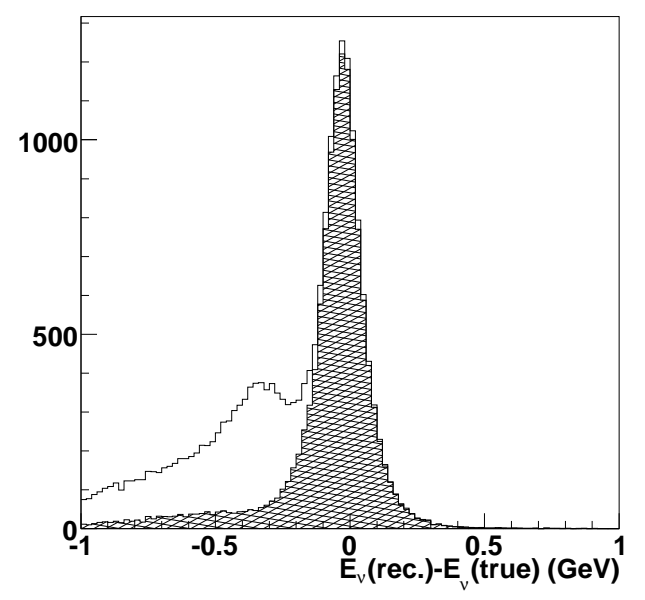

Figure 7.13: Difference between the reconstructed and true $E_{\nu}$ for the MRD stopped sample estimated with the MC simulation. Hatched histogram shows CC-QE events.

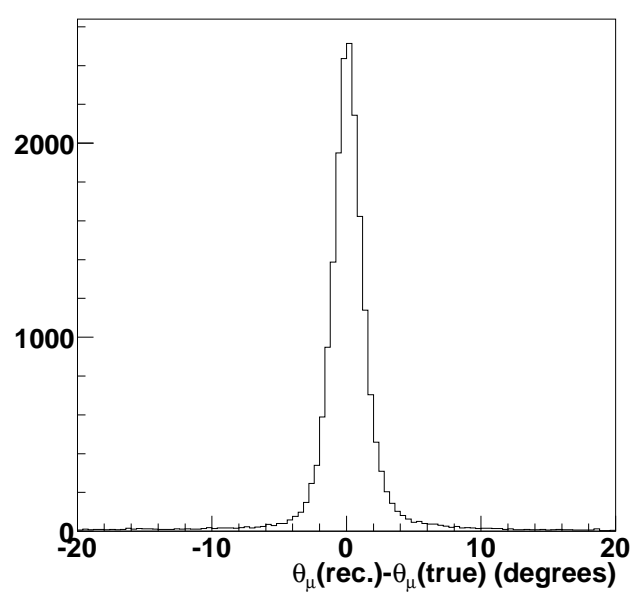

Figure 7.12: Difference between the reconstructed and true muon angles for the MRD stopped sample estimated with the MC simulation.

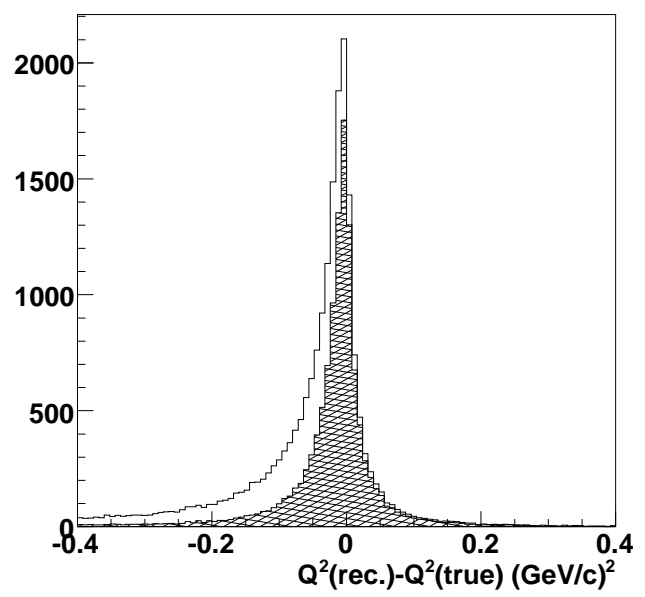

Figure 7.14: Difference between the reconstructed and true $Q^{2}$ for the MRD stopped sample estimated with the MC simulation. Hatched histogram shows CC-QE events. 


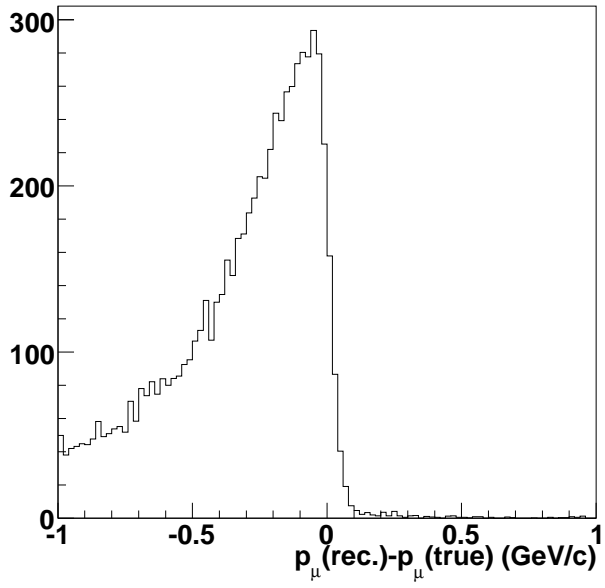

Figure 7.15: Difference between the reconstructed and true muon momenta for the MRD penetrated sample estimated with the MC simulation.

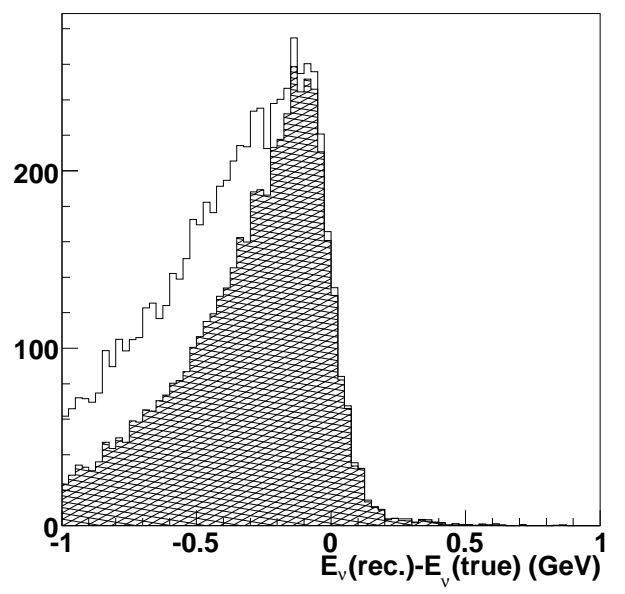

Figure 7.17: Difference between the reconstructed and true $E_{\nu}$ for the MRD stopped sample estimated with the MC simulation. Hatched histogram shows CC-QE events.

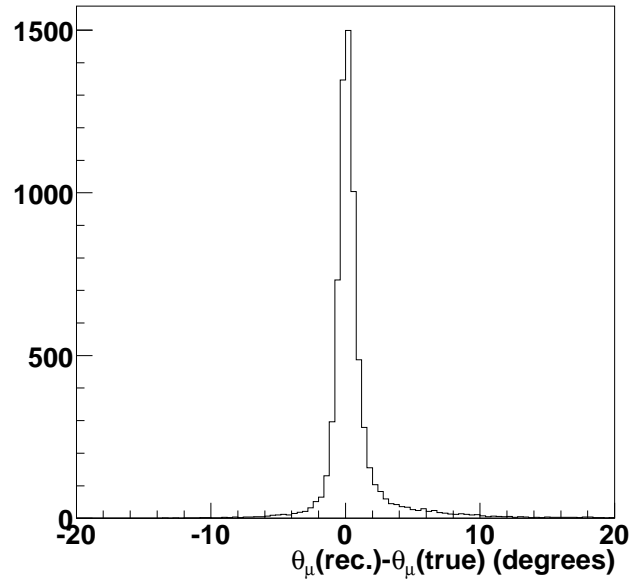

Figure 7.16: Difference between the reconstructed and true muon angles for the MRD penetrated sample estimated with the MC simulation.

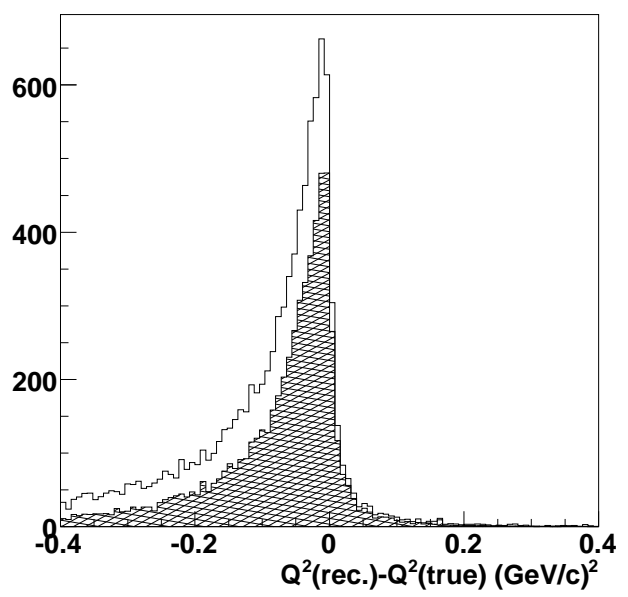

Figure 7.18: Difference between the reconstructed and true $Q^{2}$ for the MRD stopped sample estimated with the MC simulation. Hatched histogram shows CC-QE events. 


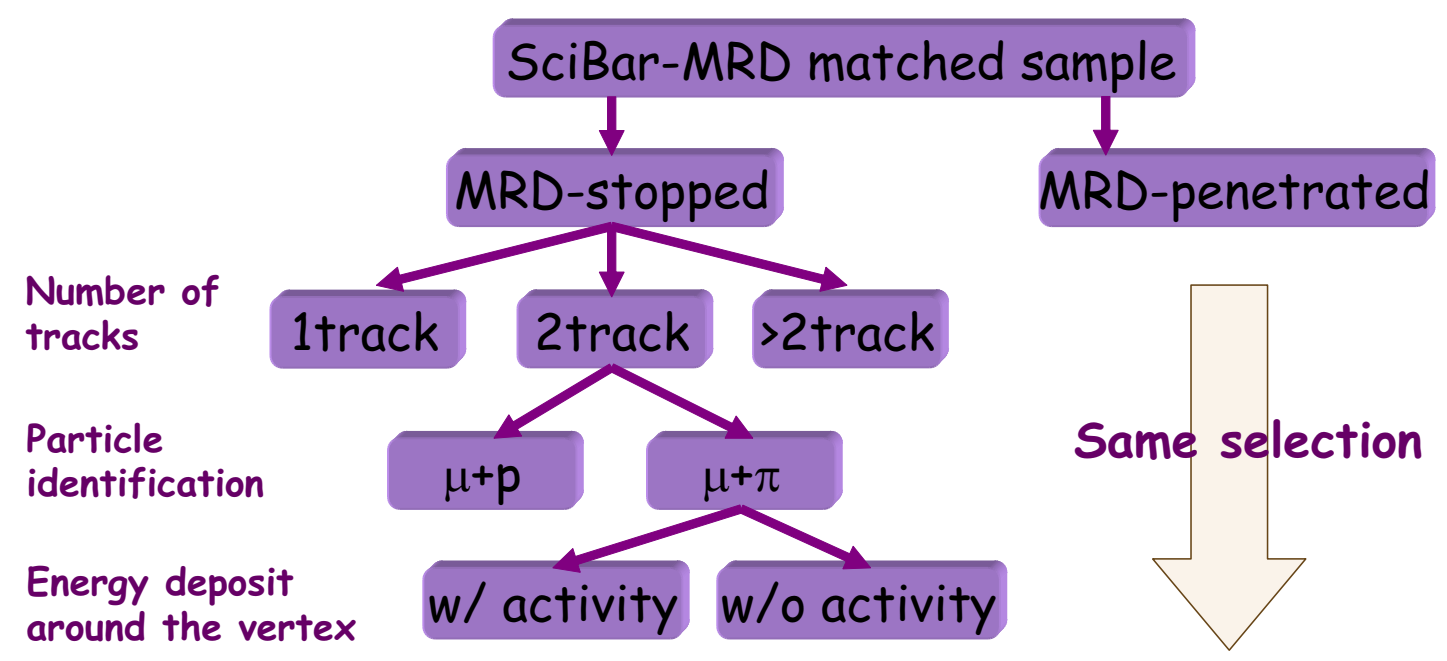

Figure 7.19: Flow chart of event classification.

\subsection{Event classification}

The MRD stopped and MRD penetrated samples are further divided into sub-samples with the same selection criteria. The event classification is based on the number of vertexmatched tracks, particle identification, and activity around the vertex. Figure 7.19 shows a flow chart of the event classification.

\subsubsection{Number of vertex-matched tracks}

Once a muon track and the neutrino interaction vertex are reconstructed, we search for other tracks originating from the vertex. The track edge distance is defined as the 3D distance between the vertex and the closer edge of another reconstructed track. Figure 7.20 shows the distribution of the track edge distance for all reconstructed tracks in the MRD stopped sample. Tracks whose edge distance is within $10 \mathrm{~cm}$ are selected to be the vertex-matched tracks.

Figure 7.21 shows the number of vertex-matched tracks for the MRD stopped sample. For the MC simulation, the contributions from charged current coherent pion, charged current resonant pion, CC-QE, and other interactions are shown separately. The "other" category contains charged current deep inelastic interactions, neutral current interactions, and antineutrino interactions, with a small contamination of neutrino events which occur in the EC and MRD. Most events are reconstructed as either one track or two track events.

\subsubsection{Particle identification}

The SciBar detector has the capability to distinguish protons from muons and pions using $d E / d x$. For this purpose, we construct the particle identification variable, Muon Confidence Level (MuCL) as follows. 


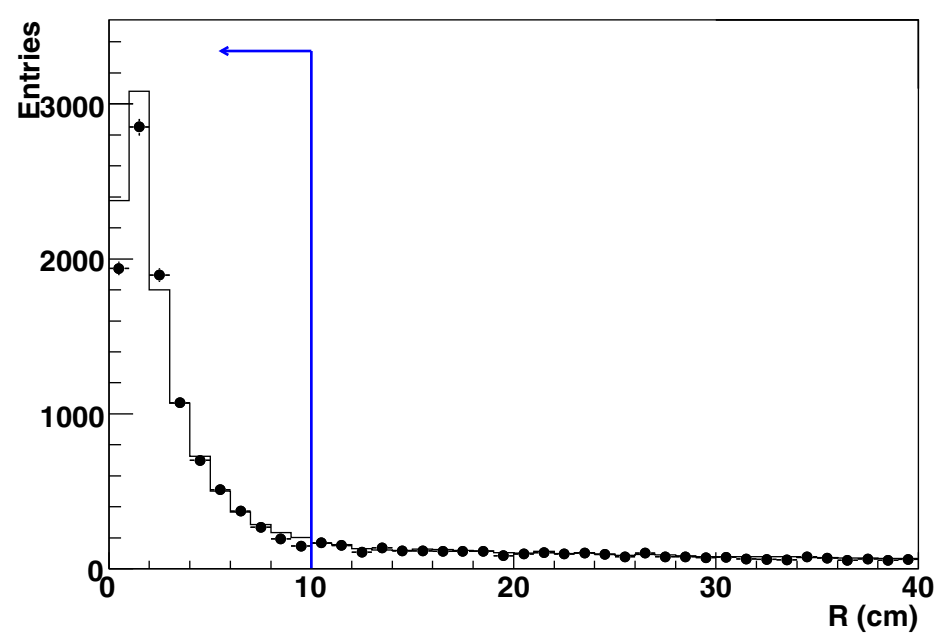

Figure 7.20: Track edge distance distribution for all reconstructed tracks in the MRD stopped sample.

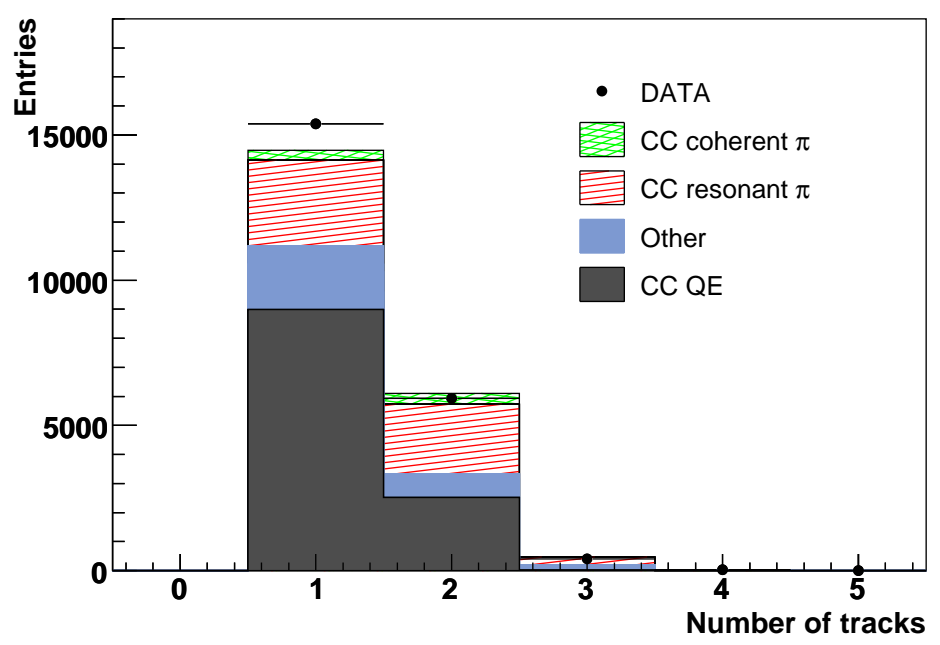

Figure 7.21: Number of tracks from the vertex for the MRD stopped sample.

\section{Definition of Muon Confidence Level (MuCL)}

The first step of the particle identification is to estimate a confidence level that a particle is identified as a muon on a plane-by-plane basis. The confidence level at each plane is defined as the fraction of events in the expected $d E / d x$ distribution of muons above the observed value, $(d E / d x)_{\text {obs. }}$. The expected $d E / d x$ distribution of muons is obtained by using cosmic-ray muons as shown in Figure 7.22 (left). Figure 7.22 (right) shows the cumulative distribution function of the muon $d E / d x$ distribution, or the confidence level at each plane as a function of $(d E / d x)_{\text {obs }}$.

The next step is to combine the confidence levels obtained from all the planes penetrated by the track to form a total confidence level. Assuming the confidence level at 

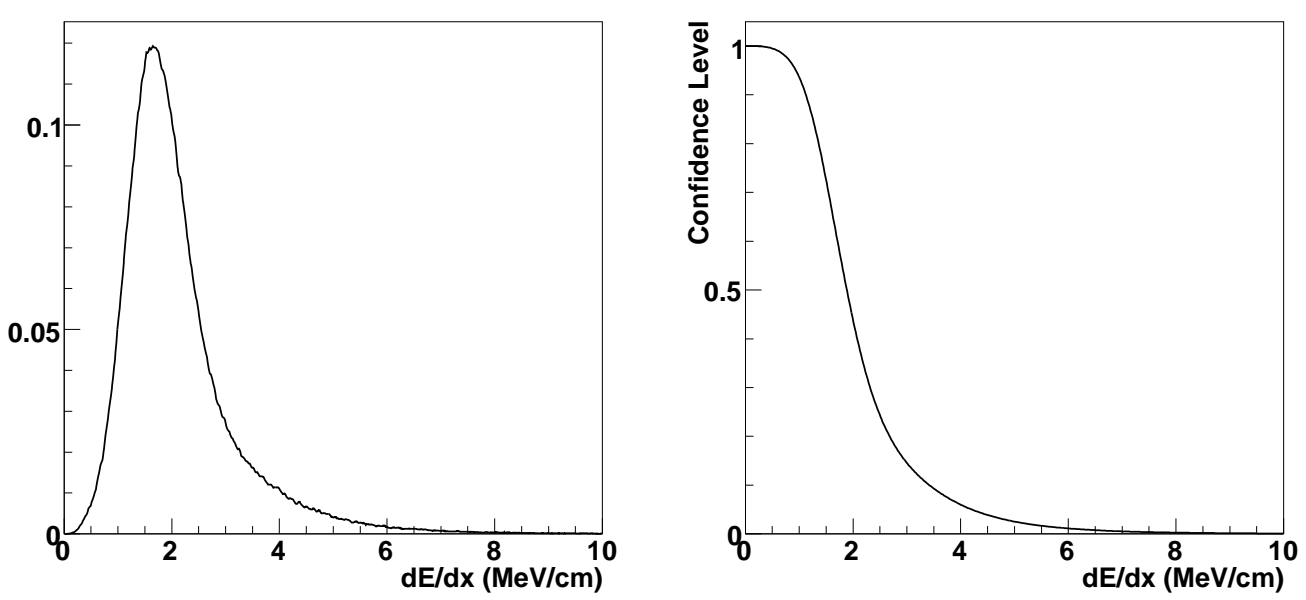

Figure 7.22: Expected $d E / d x$ distribution of a cosmic-ray muon (left) and confidence level as a function of $d E / d x$ observed in a scintillator plane (right).

each layer is independent, the MuCL is calculated as

$$
\mathrm{MuCL}=P \times \sum_{i=0}^{n-1} \frac{(-\ln P)^{i}}{i !},
$$

where $n$ is the number of planes penetrated by the track, $P=\prod_{i=1}^{n} \mathrm{CL}_{i}, \mathrm{CL}_{i}$ is the confidence level at the $i$-th plane.

In reality, the measurement is affected by inefficiency, noise hits and the energy deposition by the other particles. In order to avoid the effects of the cross talk of MA-PMT and the inefficiency of scintillator, planes with less than 6 p.e. are not used for the MuCL calculation. In case of a track overlapped with the other tracks in one view, whole hits in the overlapping view are not used. Then, we sort CLs for each plane in a track sequentially and truncate CLs of 10\% from the larger ones and 50\% from the smaller ones, and calculate MuCL using the rest of CLs.

\section{Performance of $\mu / p$ identification}

The performance of the particle identification is demonstrated using muon and proton enriched control samples. To select muon candidates for this study, we select SciBar-MRD matched tracks. According to the MC simulation, the sample is $94.7 \%$ pure muons with a small contamination of protons and charged pions. For proton candidates, we select the second track in a CC-QE enriched sample made by cutting on a kinematic variable described later. The fraction of protons in the sample is $92.1 \%$, estimated with the MC simulation. The contamination of charged pions and muons are estimated to be $5.5 \%$ and $1.6 \%$, respectively.

Figure 7.23 shows the $d E / d x$ distributions of muon and proton enriched samples. The predicted distributions of true muon and proton tracks are shown as hatched histograms. The MC distributions well reproduce the data. The MuCL distributions for the muon enriched sample and the proton enriched sample are shown in Fig. 7.24. Proton candidates are clearly separated from muon candidates. Tracks with MuCL greater than 0.05 

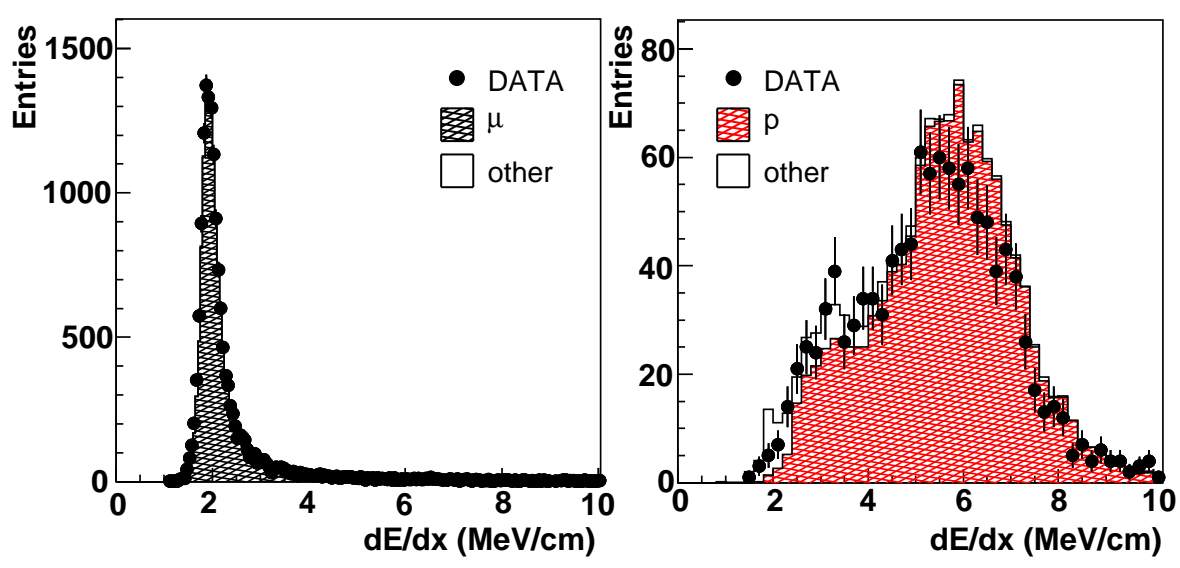

Figure 7.23: $d E / d x$ of the muon track (left) and proton track (right).
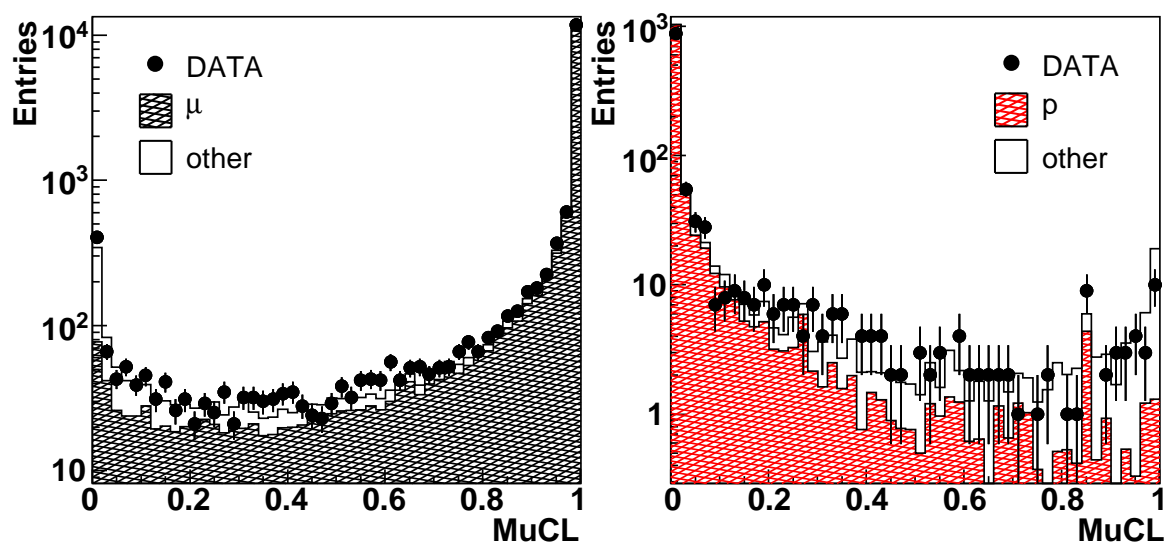

Figure 7.24: MuCL of the muon track (left) and proton track (right).

are considered muon-like (or pion-like) and the others are classified as proton-like. The probability of misidentification is estimated to be $1.1 \%$ for muons and $12 \%$ for protons, averaged over track length in the muon and proton enriched samples.

Separation of the $\mu+p$ and $\mu+\pi$ samples

The two track sample is further divided based on the particle identification variable. We first require that the MuCL of the SciBar-MRD matched track is greater than 0.05 to reject events with a proton penetrating into the MRD. Then the second track in the event is classified as a pion-like or a proton-like track with the same MuCL threshold. Figure 7.25 shows the contributions to the second track from true proton, pion, muon, and electron tracks as predicted by the MC simulation.

\subsubsection{Vertex activity}

In a charged current resonant pion event, $\nu p \rightarrow \mu^{-} p \pi^{+}$, the proton is often not reconstructed due to its low energy, and thus the event is identified as a $\mu+\pi$ event. To separate charged current coherent pion events from charged current resonant pion events, 


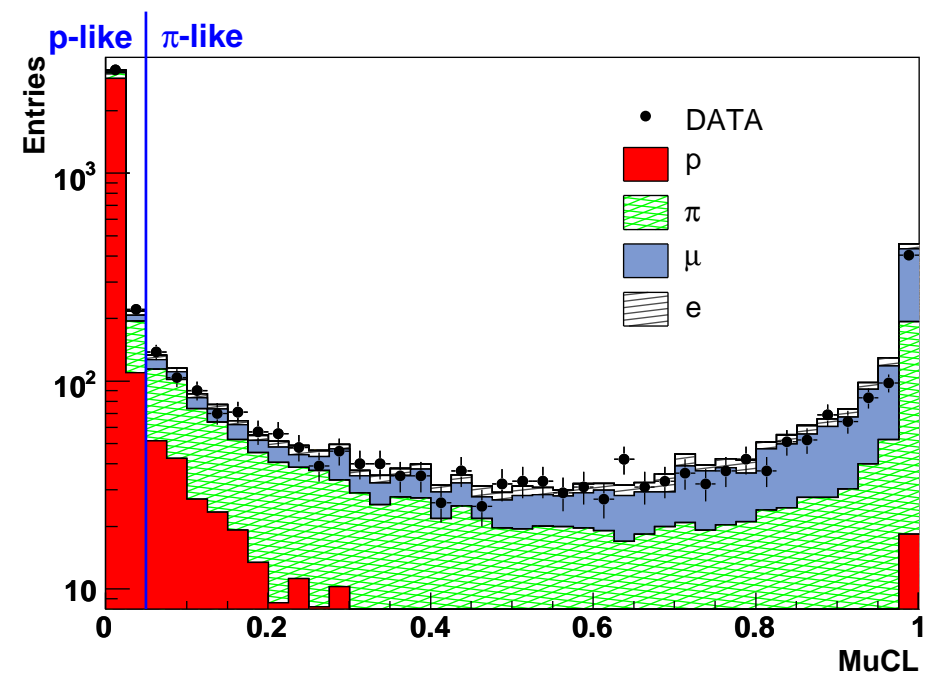

Figure 7.25: MuCL of the second track for two-track events in the MRD stopped sample.

the additional protons with momentum below the tracking threshold are detected by their large energy deposition around the vertex, so-called vertex activity. We search for the maximum deposited energy in a strip around the vertex, an area of $12.5 \mathrm{~cm} \times 12.5 \mathrm{~cm}$ in both views.

Figure 7.26 shows the maximum energy for $\mu+\pi$ events in the MRD stopped sample. A peak around $6 \mathrm{MeV}$ corresponds to the energy deposited in the strip containing the vertex by two minimum ionizing particles, and a high energy tail is mainly due to the low energy proton. To simulate such protons, we consider re-interactions of nucleons in the nucleus using the NEUT simulation as described in Section 4.2.2 as well as ones outside the nucleus using GEANT4 (described in Section 5.6.2). De-excitation gammarays from the carbon nucleus do not affect the distribution since most of the gamma-rays first interact outside the vertex region. Events with energy deposition more than $10 \mathrm{MeV}$ are considered to have activity at the vertex. Charged current coherent pion candidates are extracted from the $\mu+\pi$ events without vertex activity.

\subsection{MC tuning}

Four sub-samples, the one track events, $\mu+p$ events, $\mu+\pi$ events with vertex activity and $\mu+\pi$ events without vertex activity in the MRD stopped sample are used for constraining systematic uncertainties in the MC simulation. In this section, we describe the MC tuning in detail.

\subsubsection{Overview}

The MC simulation includes systematic uncertainties due to the detector response, nuclear effects, neutrino interaction models, and neutrino beam spectrum, and these uncertainties affect background estimation. For example, the uncertainties due to the detector response such as the crosstalk effect and the scintillator quenching affect event 


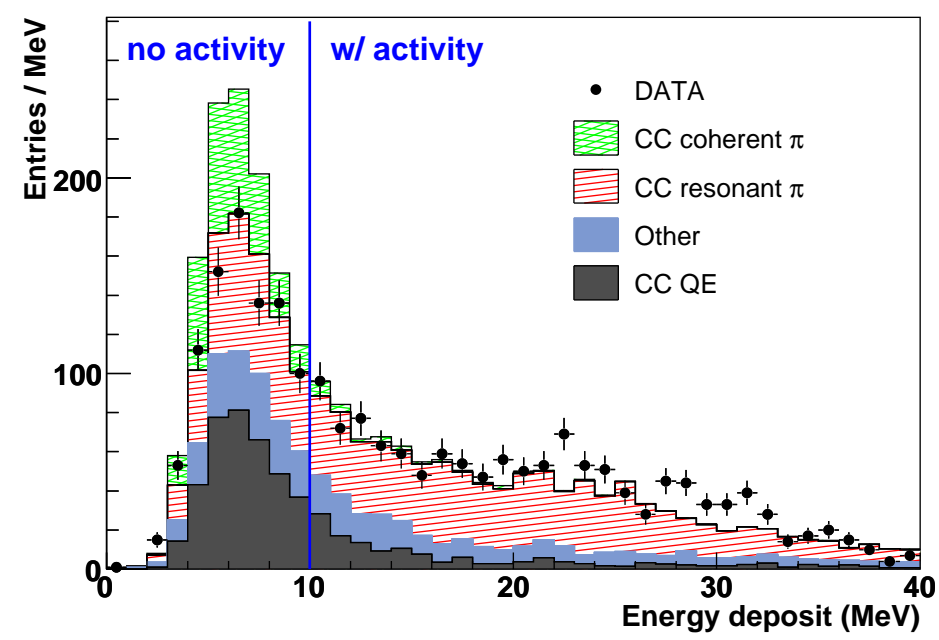

Figure 7.26: Maximum deposited energy in a strip around the vertex for the $\mu+\pi$ events in the MRD stopped sample.

reconstruction and thus cause migrations of events between sub-samples. The uncertainty in the muon momentum scale affects the shape of the reconstructed $Q^{2}$ distribution. The uncertainties due to the nuclear effects such as pion absorption and nucleon re-scattering also cause the event migration. The uncertainties in the resonant pion production and deep inelastic scattering cross sections change the amount of the background in the coherent pion event sample. All the sources of systematic uncertainty are summarized in Section 7.7.

Figure 7.27 shows reconstructed muon momenta and angles with respect to the neutrino beam before the MC tuning for the one track, $\mu+p$, and $\mu+\pi$ events with and without vertex activity. Figure 7.28 shows the reconstructed $Q^{2}$ distributions of these four sub-samples. In order to constrain these uncertainties, the MC distributions of the reconstructed $Q^{2}$ are fitted to the distributions of the four aforementioned data samples: the one track events, $\mu+p$ events, $\mu+\pi$ events with and without vertex activity in the MRD stopped sample. Each reconstructed $Q^{2}$ distribution is binned into the size of $0.05(\mathrm{GeV} / c)^{2}$ up to $1(\mathrm{GeV} / c)^{2}$.

\subsubsection{Fitting parameters}

We introduce eight fitting parameters; the normalization factor of the MRD stopped sample $\left(R_{\text {norm }}\right)$, the resonant pion scale factor $\left(R_{\text {res }}\right)$, the scale factor of other non-QE interactions $\left(R_{\text {other }}\right)$, the ratio of the number of two track events to the number of one track events $\left(R_{2 \operatorname{trk} / 1 \text { trk }}\right)$, the ratio of the number of $\mu+p$ events to the number of $\mu+\pi$ events $\left(R_{p / \pi}\right)$, the ratio of the number of low vertex activity $\mu+\pi$ events to the number of high vertex activity $\mu+\pi$ events $\left(R_{\text {act }}\right)$, the muon momentum scale $\left(R_{\text {pscale }}\right)$, and a charged current quasi-elastic Pauli-suppression parameter $\kappa$. All parameters are ratios to nominal values in the $\mathrm{MC}$ simulation, i.e. all parameters are set to 1 in the default $\mathrm{MC}$ simulation.

The parameters $R_{2 t r k / 1 t r k}, R_{p / \pi}$, and $R_{\text {act }}$ represent possible migrations between sub- 
$\mathbf{P}_{\mu}$

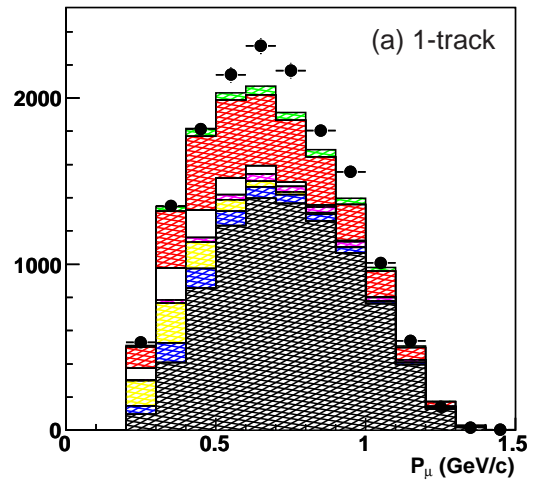

$\mathbf{P}_{\mu}$

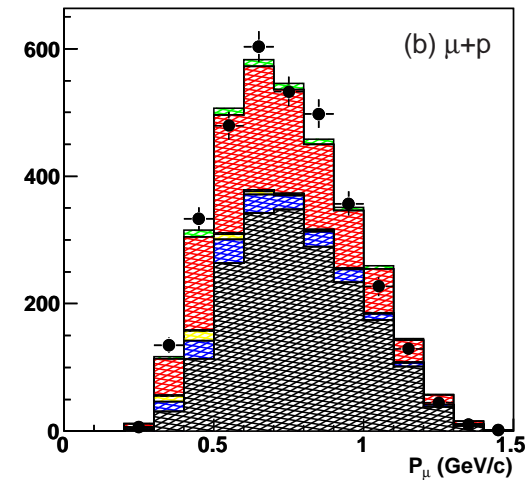

$\underline{\mathbf{P}_{\mu}}$

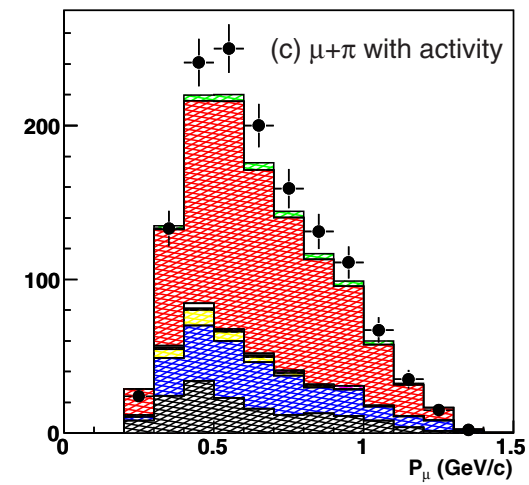

$\mathbf{P}_{\mu}$

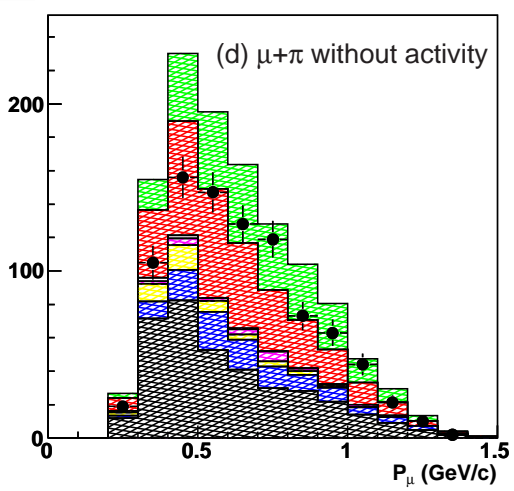

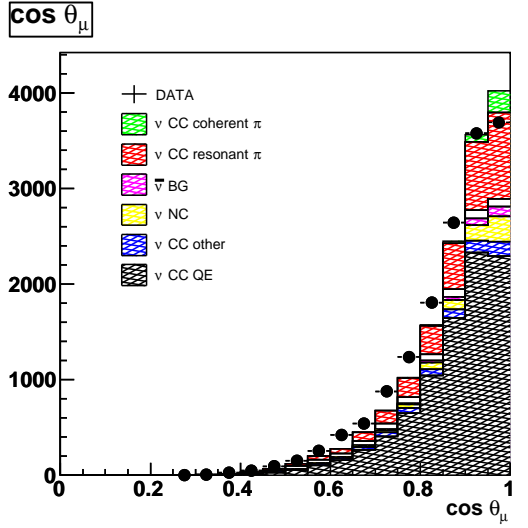

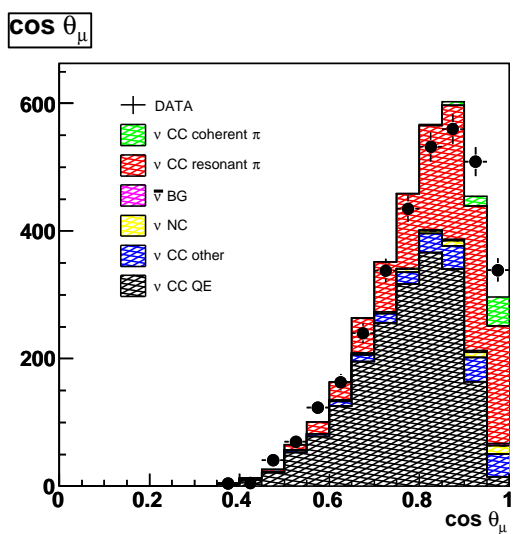

$\cos \theta_{\mu}$
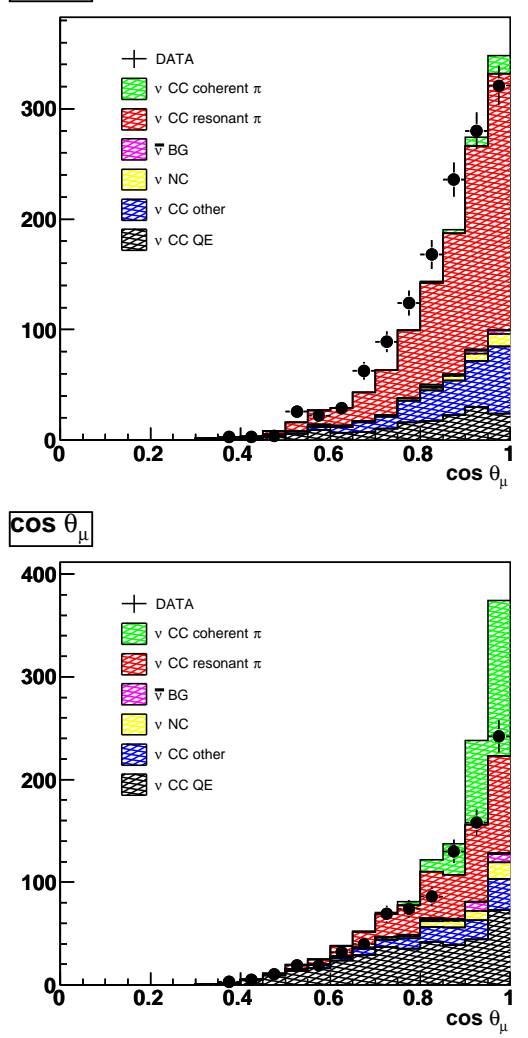

Figure 7.27: Reconstructed muon momentum and angle before fitting for (a) the one track events, (b) $\mu+p$ events, (c) $\mu+\pi$ events with activity, and (d) $\mu+\pi$ events without activity. 

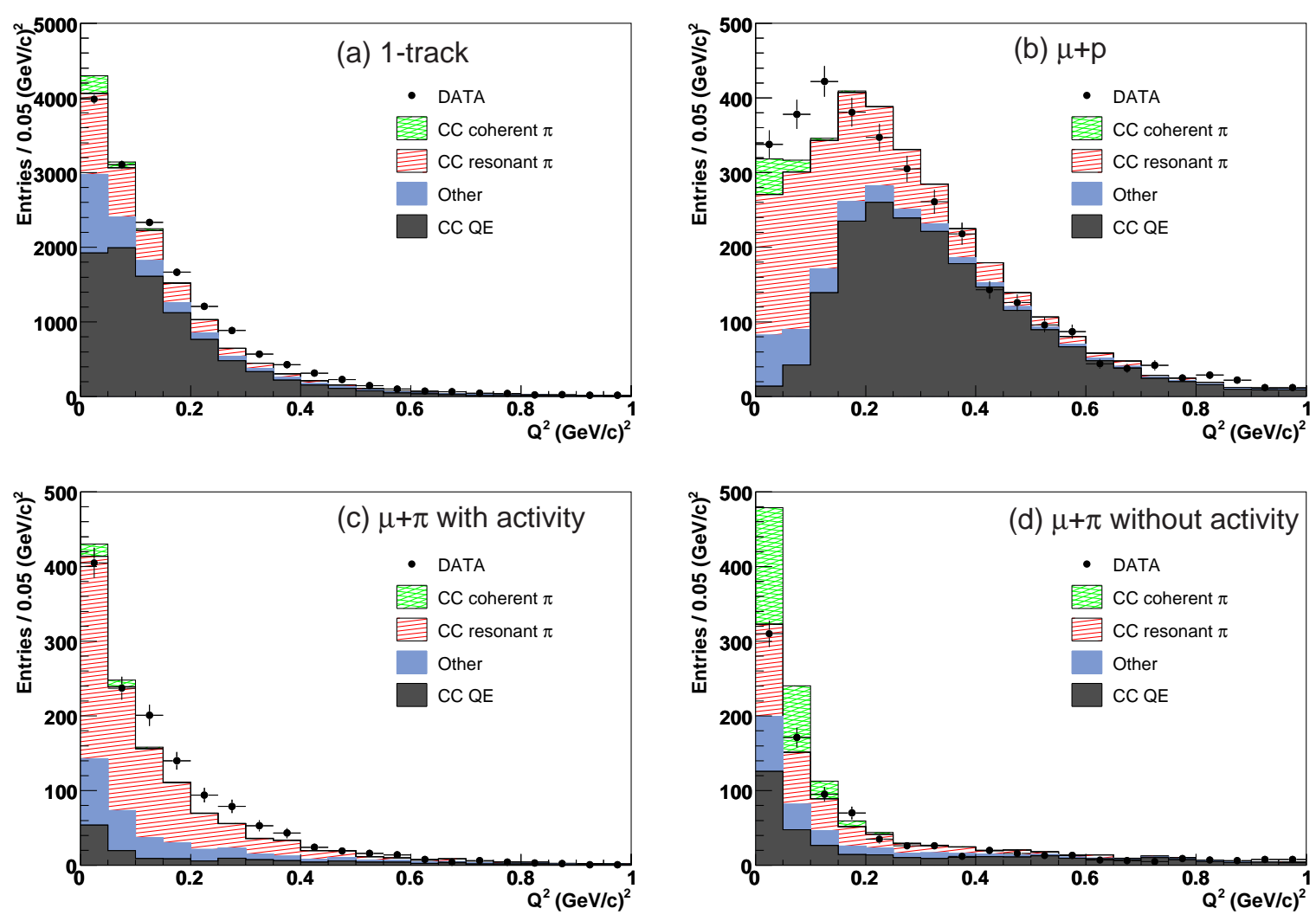

Figure 7.28: Reconstructed $Q^{2}$ before fitting for (a) the one track events, (b) $\mu+p$ events, (c) $\mu+\pi$ events with activity, and (d) $\mu+\pi$ events without activity.

samples due to systematic uncertainties. The parameter $R_{\text {pscale }}$ changes the scale of the reconstructed muon momentum for the MC simulation.

The parameter $\kappa(\kappa \geq 1)$, which was first introduced by MiniBooNE [64], controls the strength of Pauli-blocking by changing the lower bound of integration over initial state nucleon energy within the relativistic Fermi gas model:

$$
E_{\mathrm{lo}}=\kappa\left(\sqrt{p_{F}^{2}+m_{p}^{2}}-\omega+E_{B}\right)
$$

where $p_{F}$ is the Fermi surface momentum, $m_{p}$ is the out-going proton mass, $\omega$ is the energy transfer, and $E_{B}$ is the nuclear binding energy. Thus, the parameter $\kappa$ suppresses low $Q^{2}$ charged current quasi-elastic events. We employ this parameter in the fitting because a deficit of data is found at low $Q^{2}$ in the one track sample where the charged current quasi-elastic events are dominant.

\subsubsection{Definition of $\chi^{2}$}

The $\chi^{2}$ function to be minimized is given by

$$
\chi^{2}=\chi_{\text {dist }}^{2}+\chi_{\text {sys }}^{2} .
$$


The term $\chi_{\text {dist }}^{2}$ is calculated using a binned likelihood defined as [106]:

$$
\begin{aligned}
\chi_{\text {dist }}^{2} & =-2 \sum_{i, j} \ln \frac{P\left(N_{i j}^{\text {obs }} ; N_{i j}^{\mathrm{exp}}\right)}{P\left(N_{i j}^{\mathrm{obs}} ; N_{i j}^{\mathrm{obs}}\right)} \\
& =2 \sum_{i, j}\left(N_{i j}^{\mathrm{exp}}-N_{i j}^{\mathrm{obs}}+N_{i j}^{\mathrm{obs}} \times \ln \frac{N_{i j}^{\mathrm{obs}}}{N_{i j}^{\mathrm{exp}}}\right),
\end{aligned}
$$

where $P(n, \nu)$ is the Poisson probability of finding $n$ events with a expectation value $\nu$, expressed as

$$
P(n, \nu)=\frac{\nu^{n} e^{-\nu}}{n !}
$$

$N_{i j}^{\text {obs }}$ and $N_{i j}^{\text {exp }}$ are the observed and expected number of events in the $i$-th $Q^{2}$ bin in subsample $j$ ( $j=$ one track, $\mu+p, \mu+\pi$ with high and low vertex activity), respectively. The expected number of events for each sample is given by:

$$
\begin{aligned}
& N_{i, 1 \mathrm{trk}}^{\mathrm{exp}}=R_{\mathrm{norm}} \cdot\left[n_{i, 1 \mathrm{trk}}^{\mathrm{QE}}+R_{\mathrm{res}} n_{i, 1 \mathrm{trk}}^{\text {res }}+R_{\mathrm{other}} n_{i, 1 \mathrm{trk}}^{\text {other }}\right] \\
& N_{i, \mu p}^{\exp }=R_{\text {norm }} \cdot R_{2 \operatorname{trk} / 1 \mathrm{trk}} \cdot R_{p / \pi} \cdot\left[n_{i, \mu p}^{\mathrm{QE}}+R_{\mathrm{res}} n_{i, \mu p}^{\mathrm{res}}+R_{\mathrm{other}} n_{i, \mu p}^{\text {other }}\right] \\
& N_{i, \mu \pi \mathrm{H}}^{\text {exp }}=R_{\text {norm }} \cdot R_{2 \operatorname{trk} / 1 \text { trk }} \cdot\left[n_{i, \mu \pi \mathrm{H}}^{\mathrm{QE}}+R_{\text {res }} n_{i, \mu \pi \mathrm{H}}^{\text {res }}+R_{\text {other }} n_{i, \mu \pi \mathrm{H}}^{\text {other }}\right] \\
& N_{i, \mu \pi \mathrm{L}}^{\text {exp }}=R_{\text {norm }} \cdot R_{2 \text { trk } / 1 \text { trk }} \cdot R_{\text {act }} \cdot\left[n_{i, \mu \pi \mathrm{L}}^{\mathrm{QE}}+R_{\text {res }} n_{i, \mu \pi \mathrm{L}}^{\text {res }}+R_{\text {other }} n_{i, \mu \pi \mathrm{L}}^{\text {other }}\right]
\end{aligned}
$$

where $n_{i, j}^{\mathrm{QE}}, n_{i, j}^{\mathrm{res}}, n_{i, j}^{\text {other }}$ are the number of charged current quasi-elastic, charged current resonant pion, and other events in each bin in each subsample, respectively. $R_{\text {pscale }}$ and $\kappa$ do not appear explicitly in these equations, but $R_{\text {pscale }}$ causes migration between $Q^{2}$ bins and $\kappa$ changes $n_{i, j}^{\mathrm{QE}}$.

The term $\chi_{\text {sys }}^{2}$, added to constrain systematic parameters, is calculated as:

$$
\chi_{\text {sys }}^{2}=\left(\boldsymbol{P}_{\text {sys }}-\boldsymbol{P}_{\mathbf{0}}\right) \boldsymbol{V}^{-1}\left(\boldsymbol{P}_{\text {sys }}-\boldsymbol{P}_{\mathbf{0}}\right)
$$

where $\boldsymbol{P}_{\boldsymbol{s y s}}$ represents the set of systematic parameters and $\boldsymbol{P}_{\mathbf{0}}$ is the set of parameter values before fitting, expressed as:

$$
\boldsymbol{P}_{\text {sys }}=\left(\begin{array}{c}
R_{\mathrm{res}} \\
R_{2 \mathrm{trk} / 1 \mathrm{trk}} \\
R_{p / \pi} \\
R_{\mathrm{pscale}}
\end{array}\right) \quad, \quad \boldsymbol{P}_{\mathbf{0}}=\left(\begin{array}{c}
1 \\
1 \\
1 \\
1
\end{array}\right)
$$

$\boldsymbol{V}$ is a covariance matrix estimated by considering the possible variations due to systematic uncertainties in the detector responses, nuclear effects, neutrino interaction models, and neutrino beam spectrum. $R_{\text {norm }}, R_{\text {other }}, R_{\text {act }}$, and $\kappa$ are unconstrained in the fit.

\subsubsection{Covariance matrix}

The next step is to build a covariance matrix to take into account correlations between the systematic parameters. We prepare several MC event sets by changing each underlying physics parameter, i.e. the source of systematic uncertainty, by $\pm 1 \sigma$. For the 
systematic uncertainties due to detector response, the amount of crosstalk, single photoelectron resolution of the MA-PMT, the scintillator quenching effect are varied within their measured uncertainties. The hit threshold for tracking is changed by \pm 0.4 photoelectron. For uncertainties due to nuclear effects, the cross sections of pion absorption and pion inelastic scattering in the nucleus are separately varied by $\pm 30 \%$, the cross section of nucleon rescattering in the nucleus is varied by $\pm 10 \%$. The Fermi momentum of nucleons are varied by $\pm 5 \mathrm{MeV} / c$. For the systematic uncertainties due to neutrino interaction models, the axial-vector mass for both quasi-elastic and resonant pion interactions is changed from $1.21 \mathrm{GeV} / c^{2}$ to $1.11 \mathrm{GeV} / c^{2}$ simultaneously, and the resonant pion production and deep inelastic scattering cross sections are varied by $\pm 20 \%$ and $\pm 30 \%$, respectively. Further description of each source is given in Section 7.7.

The covariance between two systematic parameters $p_{i}$ and $p_{j}$ is calculated as:

$$
V_{i j} \equiv \operatorname{cov}\left[p_{i}, p_{j}\right]=\sum_{\text {source }} \frac{\left.\Delta p_{i} \Delta p_{j}\right|_{+}+\left.\Delta p_{i} \Delta p_{j}\right|_{-}}{2}
$$

where $\left.\Delta p_{i} \Delta p_{j}\right|_{+(-)}$is the product of variations of two parameters when the underlying physics parameter is increased (decreased) by the size of its uncertainty. The covariance matrix is estimated to be:

$$
\boldsymbol{V}=\left(\begin{array}{cccc}
(0.20)^{2} & -(0.09)^{2} & +(0.10)^{2} & 0 \\
-(0.09)^{2} & (0.09)^{2} & -(0.07)^{2} & 0 \\
+(0.10)^{2} & -(0.07)^{2} & (0.15)^{2} & 0 \\
0 & 0 & 0 & (0.02)^{2}
\end{array}\right)
$$

\subsubsection{Fitting results}

Events with $Q_{\mathrm{rec}}^{2}<0.10(\mathrm{GeV} / c)^{2}$ in the $\mu+\pi$ sample with low activity are not included in the fit to avoid charged current coherent pion signal events. A data excess is observed in the region with $Q_{\mathrm{rec}}^{2}<0.15(\mathrm{GeV} / c)^{2}$ in the $\mu+p$ sample. Further investigation has been performed and is described in Appendix B. This study reveals that the second track in the excess events is emitted at a relatively large angle with respect to the beam direction and has large $d E / d x$, and that the events have an additional large energy deposition at the vertex. Each of these events seems to have a muon and a proton with additional activity, and therefore the excess is not expected to affect the charged current coherent pion analysis. A possible candidate for the excess is charged current resonant pion production where the pion is absorbed in the nucleus. In such an event, two or more additional nucleons should be emitted after the pion is absorbed, which is currently not simulated. The excess cannot be explained with the introduced fitting parameters, and therefore the region is not used in the fit.

A minimum point of $\chi^{2}$ is searched using the MINUIT package [107]. Fig. 7.29 shows reconstructed $Q^{2}$ after the fitting for the one track, $\mu+p$, and $\mu+\pi$ events with and without vertex activity. The MC distributions reasonably reproduce observations in the regions used for fitting. The best fit values and errors of the fitting parameters are summarized 
Table 7.1: Best fit values and errors of the fitting parameters

\begin{tabular}{lcc}
\hline \hline Parameter & Value & Error \\
\hline$R_{\text {norm }}$ & 1.103 & 0.029 \\
$R_{2 \text { trk } / 1 \text { trk }}$ & 0.865 & 0.035 \\
$R_{p / \pi}$ & 0.899 & 0.038 \\
$R_{\text {act }}$ & 0.983 & 0.055 \\
$R_{\text {pscale }}$ & 1.033 & 0.002 \\
$R_{\text {res }}$ & 1.211 & 0.133 \\
$R_{\text {other }}$ & 1.270 & 0.148 \\
$\kappa$ & 1.019 & 0.004 \\
\hline \hline
\end{tabular}

in Table 7.1. The correlation coefficient matrix is obtained as

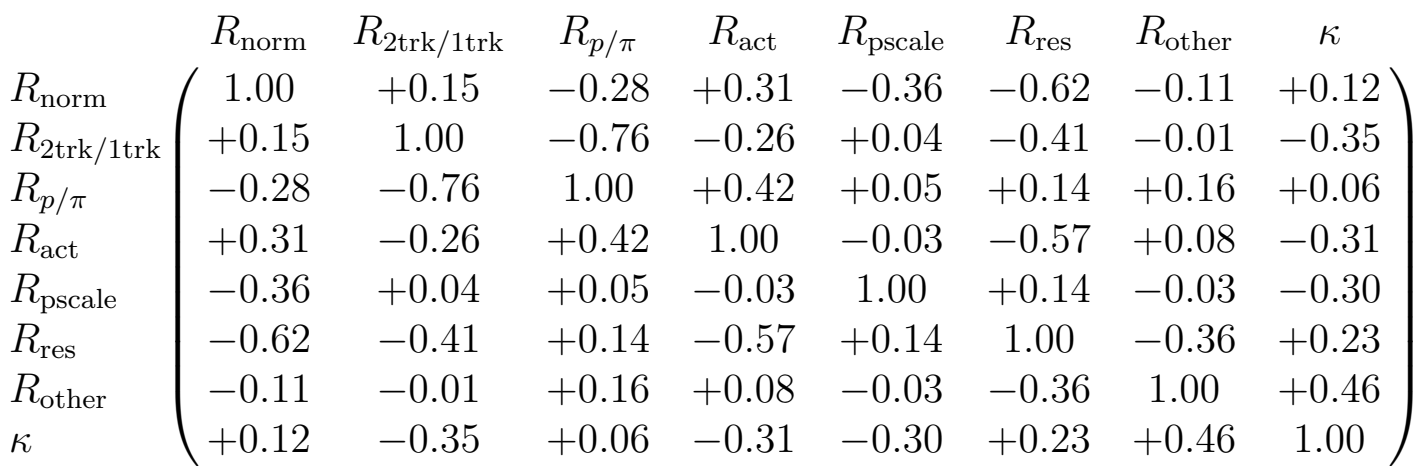

The resonant pion cross section is increased by $R_{\text {res }}$, and related with this, $R_{2 \text { trk } / 1 \text { trk }}$ is decreased to tune the ratio of the number of two track events to the number of one track events. The current knowledge of the charged current resonant pion production cross section is at a $20 \%$ level, and thus the value of $R_{\text {res }}=1.211 \pm 0.133$ is possible. The main contribution to "other" events is deep inelastic scattering, and the uncertainty in the deep inelastic scattering cross section in the default MC simulation is estimated to be $30 \%$. The obtained result of $R_{\text {other }}=1.270 \pm 0.148$ is within the uncertainty. The fitted value of $\kappa$ is consistent with MiniBooNE's result of $\kappa=1.019 \pm 0.011$. The $\chi^{2} /$ d.o.f before the fit is $473 / 75=6.31$. The $\chi^{2} /$ d.o.f after the fit is $117 / 67=1.75$.

Figure 7.30 shows reconstructed muon momenta and angles with respect to the neutrino beam after the fitting for the one track, $\mu+p$, and $\mu+\pi$ events with and without vertex activity. Other kinematic distributions after the fitting for each sub-samples are shown in Appendix C.

\subsection{Coherent pion event extraction}

Charged current coherent pion candidates are extracted from both the MRD stopped and MRD penetrated samples with the same selection criteria. In this section, we first describe the event selection for the MRD stopped sample. The event selection for the MRD penetrated sample is summarized later. 

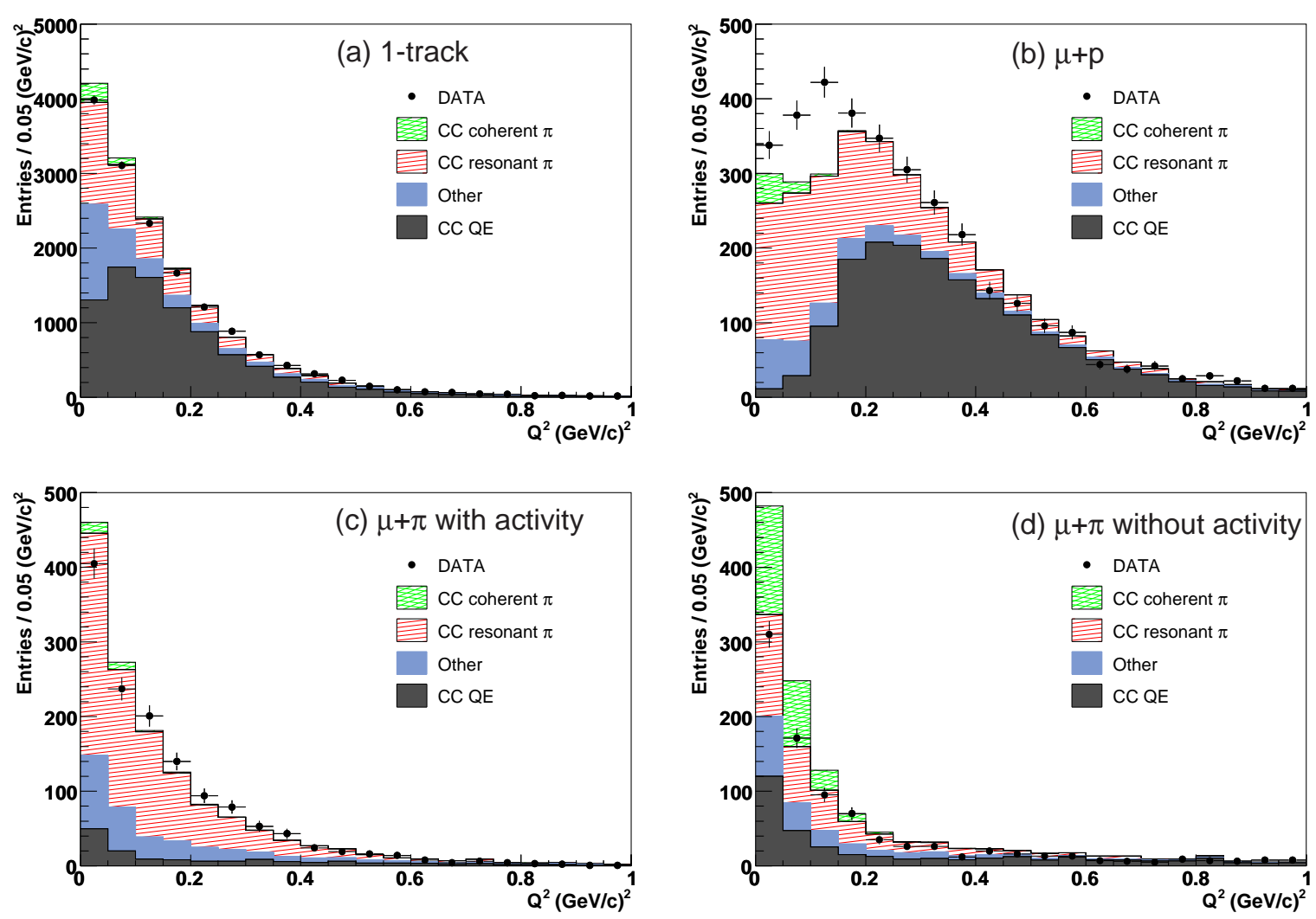

Figure 7.29: Reconstructed $Q^{2}$ after fitting for (a) the one track events, (b) $\mu+p$ events, (c) $\mu+\pi$ events with activity, and (d) $\mu+\pi$ events without activity.

\subsubsection{MRD stopped charged current coherent pion events}

\section{CC-QE rejection}

After selecting $\mu+\pi$ events which do not have vertex activity, the sample still contains charged current quasi-elastic events in which a proton is misidentified as a minimum ionizing track. We reduce this charged current quasi-elastic background by making use of kinematic information in the event. Since the charged current quasi-elastic interaction is a two-body interaction, one can predict the proton direction from the measured muon momentum $p_{\mu}$ and muon angle $\theta_{\mu}$;

$$
\vec{p}_{p}=\left(-p_{\mu x},-p_{\mu y}, E_{\nu}^{\mathrm{rec}}-p_{\mu} \cos \theta_{\mu}\right)
$$

where $p_{\mu x}$ and $p_{\mu y}$ are the projected muon momentum in the $x$ and $y$ dimension, respectively. $E_{\nu}^{\text {rec }}$ is the reconstructed neutrino energy given by Equation 7.6. For each two-track event, we define an angle called $\Delta \theta_{p}$ as the angle between the expected proton track direction given by Equation 7.22 and the observed second track direction:

$$
\cos \Delta \theta_{p}=\frac{-p_{\mu x} \tan \theta_{x z}-p_{\mu y} \tan \theta_{y z}+E_{\nu}^{r e c}-p_{\mu} \cos \theta_{\mu}}{\sqrt{\left(E_{\nu}^{r e c}\right)^{2}+p_{\mu}^{2}-2 E_{\nu}^{r e c} p_{\mu} \cos \theta_{\mu}} \sqrt{1+\tan ^{2} \theta_{x z}+\tan ^{2} \theta_{y z}}}
$$

where $\theta_{x z}$ and $\theta_{y z}$ are the second track angle in each view with respect to the beam direction. Fig. 7.31 shows the $\Delta \theta_{p}$ distribution for $\mu+\pi$ events in the MRD stopped 
$\mathbf{P}_{\mu}$

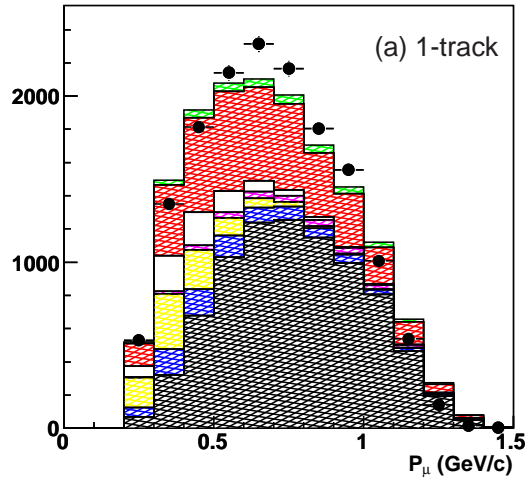

$\mathbf{P}_{\mu}$

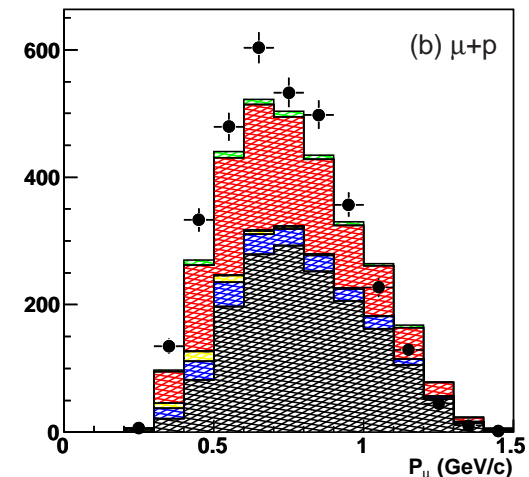

$\mathbf{P}_{\mu}$

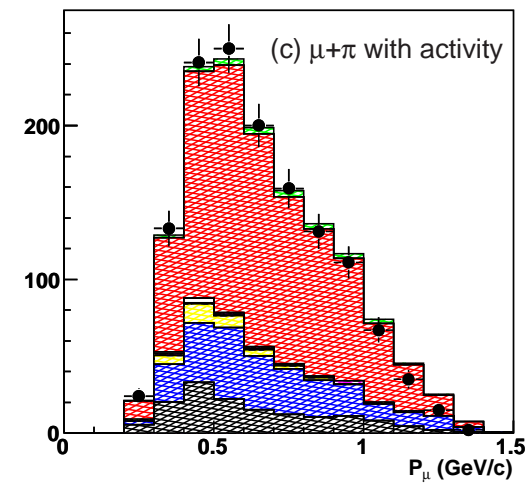

$\mathbf{P}_{\mu}$

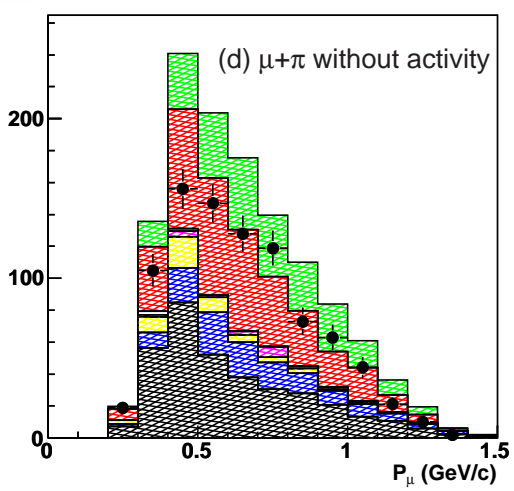

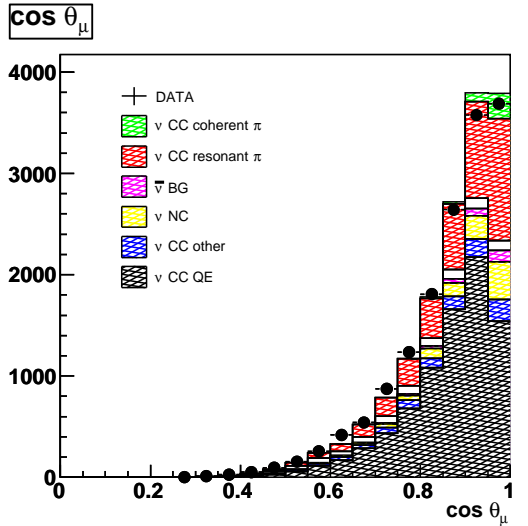
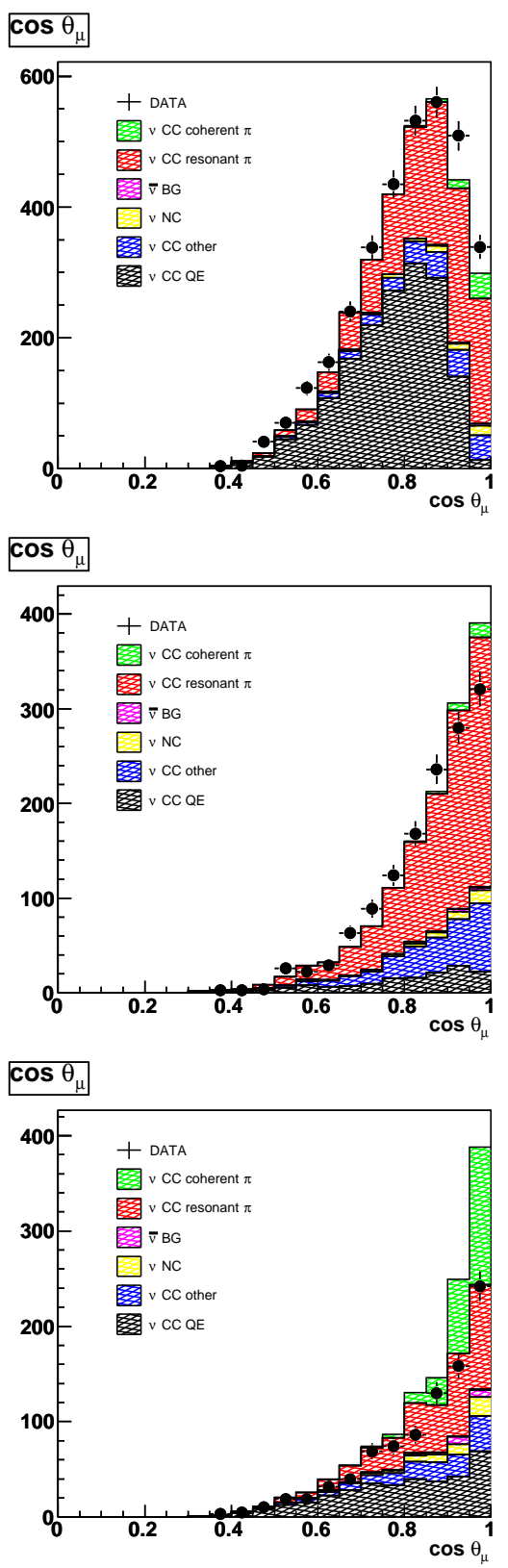

Figure 7.30: Reconstructed muon momentum and angle after fitting for (a) the one track events, (b) $\mu+p$ events, (c) $\mu+\pi$ events with activity, and (d) $\mu+\pi$ events without activity. 


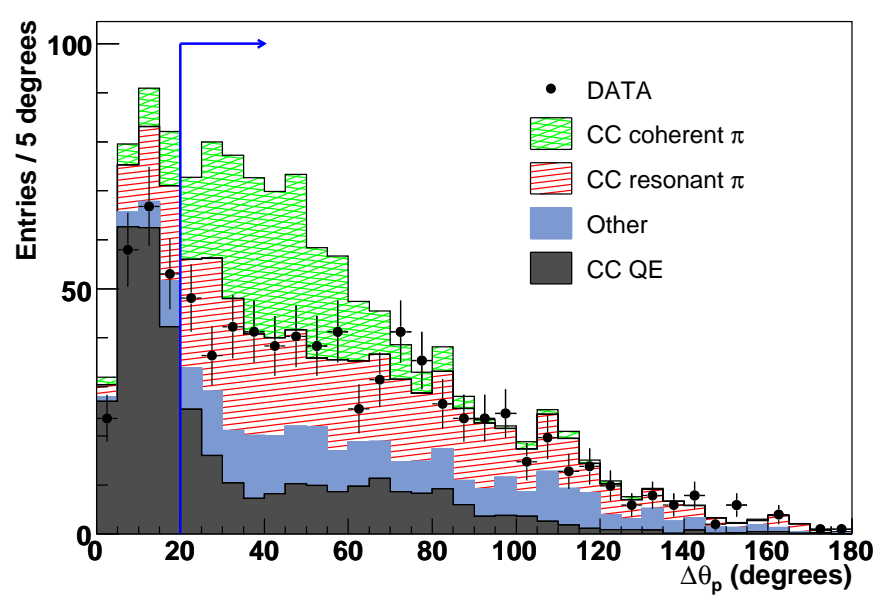

Figure 7.31: $\Delta \theta_{p}$ for the $\mu+\pi$ events in the MRD stopped sample after fitting.

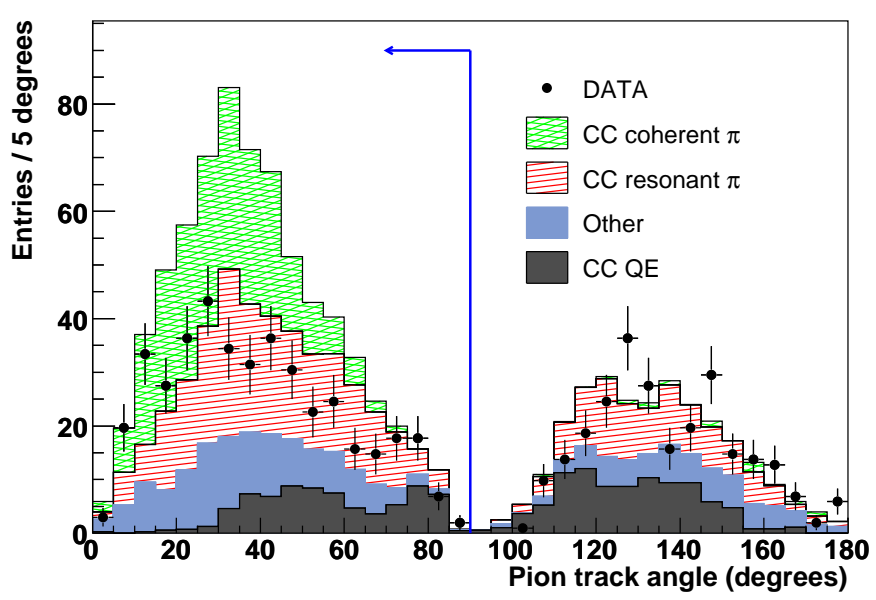

Figure 7.32: Track angle of the pion candidate with respect to the beam direction for the $\mu+\pi$ events after the charged current quasi-elastic rejection after fitting.

sample. Events with $\Delta \theta_{p}$ larger than 20 degrees are selected. With this selection, $48 \%$ of charged current quasi-elastic events in the $\mu+\pi$ sample are rejected, while $91 \%$ of charged current coherent pion events pass the cut according to the MC simulation.

\section{Pion track direction cut}

Further selections are applied in order to separate charged current coherent pion events from charged current resonant pion events which are the dominant backgrounds for this analysis. Fig. 7.32 shows the angular distribution of pion candidates with respect to the beam direction. In the case of charged current coherent pion events, both the muon and pion tracks are directed forward. Events in which the track angle of the pion candidate with respect to the beam direction is less than 90 degrees are selected. 


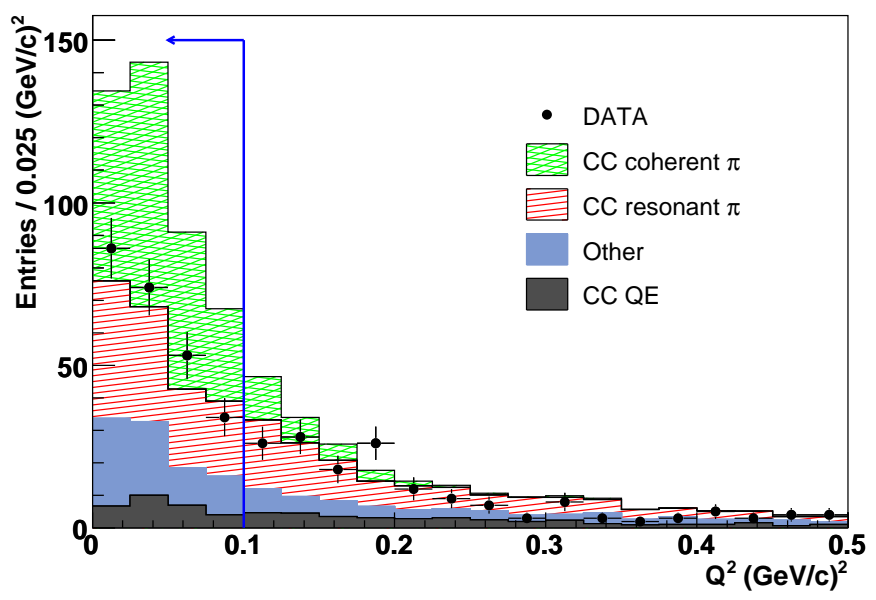

Figure 7.33: Reconstructed $Q^{2}$ for the $\mu+\pi$ events in the MRD stopped sample after the pion track direction cut and after fitting.

\section{Reconstructed $Q^{2}$ cut}

Fig. 7.33 shows the reconstructed $Q^{2}$ distribution for the $\mu+\pi$ events after the pion track direction cut. Although a charged current quasi-elastic interaction is assumed, the $Q^{2}$ of charged current coherent pion events is reconstructed with a resolution of $0.016(\mathrm{GeV} / c)^{2}$ and a shift of $-0.024(\mathrm{GeV} / c)^{2}$ according to the MC simulation as shown in Fig. 7.34. Finally, events with reconstructed $Q^{2}$ less than $0.1(\mathrm{GeV} / c)^{2}$ are selected. The charged current coherent pion event selection is summarized in Table 7.2. In the signal region, 247 charged current coherent pion candidates are observed, while the expected number of background events is $228 \pm 12$. The error comes from the errors on the fitting parameters summarized in Table 7.1, calculated as

$$
\delta N=\sqrt{\sum_{i}\left(\frac{\partial N}{\partial p_{i}} \cdot \delta p_{i}\right)^{2}+2 \sum_{i<j} \rho_{i j}\left(\frac{\partial N}{\partial p_{i}}\right)\left(\frac{\partial N}{\partial p_{i}}\right) \cdot \delta p_{i} \cdot \delta p_{j}}
$$

where $p_{i}$ represents each fitting parameter. $\delta p_{i}$ is the fitting error in the parameter $p_{i}$. Even after fitting, the reduced $\chi^{2}$ is relatively large, which indicates that the introduced parameters are not sufficient in fully reproducing the data. To take into account the incompleteness of our simulation, we enlarge the errors on the fitting parameters by a factor of $\sqrt{\chi^{2} / \text { d.o.f. }}\left(\partial N / \partial p_{i}\right) \cdot \delta p_{i}=\left|N\left(\left\langle p_{i}\right\rangle+\delta p_{i}\right)-N\left(\left\langle p_{i}\right\rangle\right)\right|$ is estimated with the $\mathrm{MC}$, where $\left\langle p_{i}\right\rangle$ is the best fit value. $\rho_{i j}$ is the correlation coefficient matrix, obtained from fitting.

The background in the final sample is dominated by charged current resonant pion production. The "other" background is comprised of $50 \%$ charged current DIS, $32 \%$ neutral current, and $18 \% \bar{\nu}_{\mu}$ events. The selection efficiency for the signal is estimated to be $10.4 \%$. 


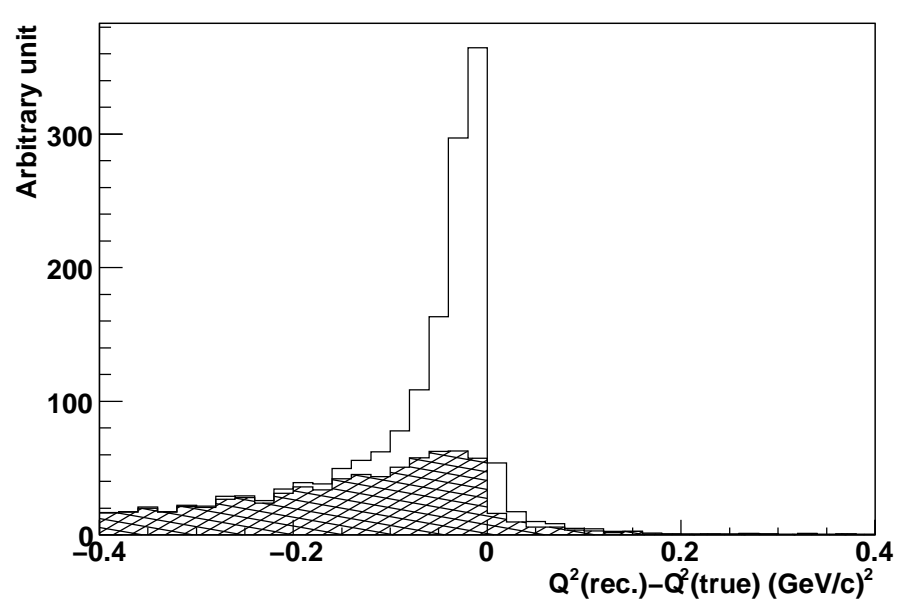

Figure 7.34: Resolution of reconstructed $Q^{2}$ for the $\mu+\pi$ events in the MRD stopped sample after the pion track direction cut. The hatched histogram shows the background contribution.

Table 7.2: Event selection summary for the MRD stopped charged current coherent pion sample. The MC simulation is normalized to the number of the SciBar-MRD matched events in data.

\begin{tabular}{lrrrr}
\hline \hline Event selection & DATA & \multicolumn{2}{r}{ MC } & Coherent $\pi$ \\
& & Signal & B.G. & Efficiency \\
\hline Generated in SciBar fid.vol. & & 1,939 & 156,766 & $100 \%$ \\
SciBar-MRD matched & 30,337 & 978 & 29,359 & $50.4 \%$ \\
\hline MRD stopped & 21,762 & 715 & 20,437 & $36.9 \%$ \\
2 track & 5,939 & 358 & 6,073 & $18.5 \%$ \\
Particle ID $(\mu+\pi)$ & 2,255 & 292 & 2,336 & $15.1 \%$ \\
Vertex activity cut & 887 & 264 & 961 & $13.6 \%$ \\
CC-QE rejection & 682 & 241 & 709 & $12.4 \%$ \\
Pion track direction cut & 425 & 233 & 451 & $12.0 \%$ \\
Reconstructed $Q^{2}$ cut & 247 & 201 & 228 & $10.4 \%$ \\
\hline \hline
\end{tabular}

\subsubsection{MRD penetrated charged current coherent pion events}

The same selection is applied to the MRD penetrated sample to extract charged current coherent pion candidates at higher energy. Fig. 7.35 shows the reconstructed $Q^{2}$ distribution of the MRD penetrated charged current coherent pion sample. The difference between the reconstructed $Q^{2}$ and true $Q^{2}$ for this sample is shown in Figure 7.36. Although only a part of the muon energy is observed, the $Q^{2}$ reconstruction performance is essentially the same because of the small muon angle. The event selection is summarized in Table 7.3. In the signal region, 57 charged current coherent pion candidates are observed, while the expected number of background events is $40 \pm 2.2$. The background in the final sample is dominated by charged current resonant pion production. The "other" 


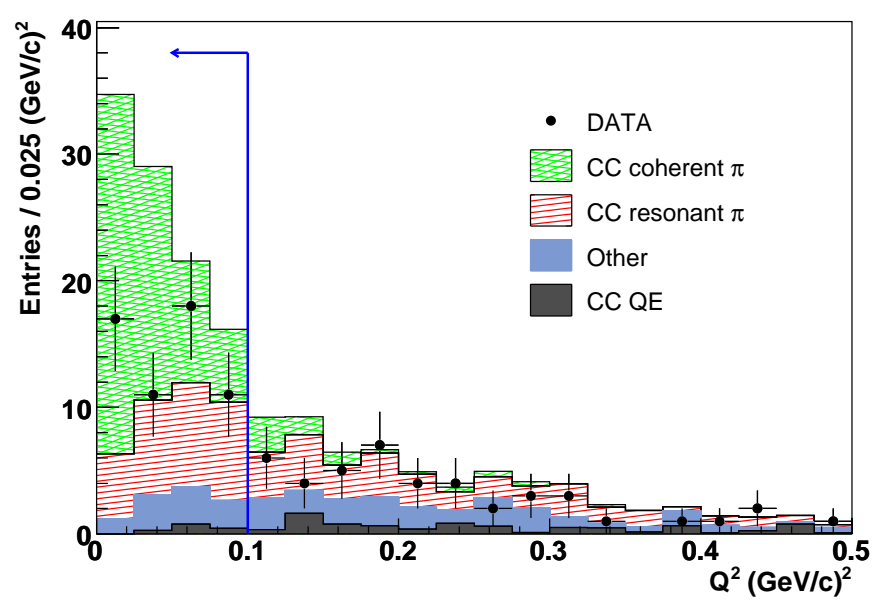

Figure 7.35: Reconstructed $Q^{2}$ for the $\mu+\pi$ events in the MRD penetrated sample after the pion track direction cut after fitting.

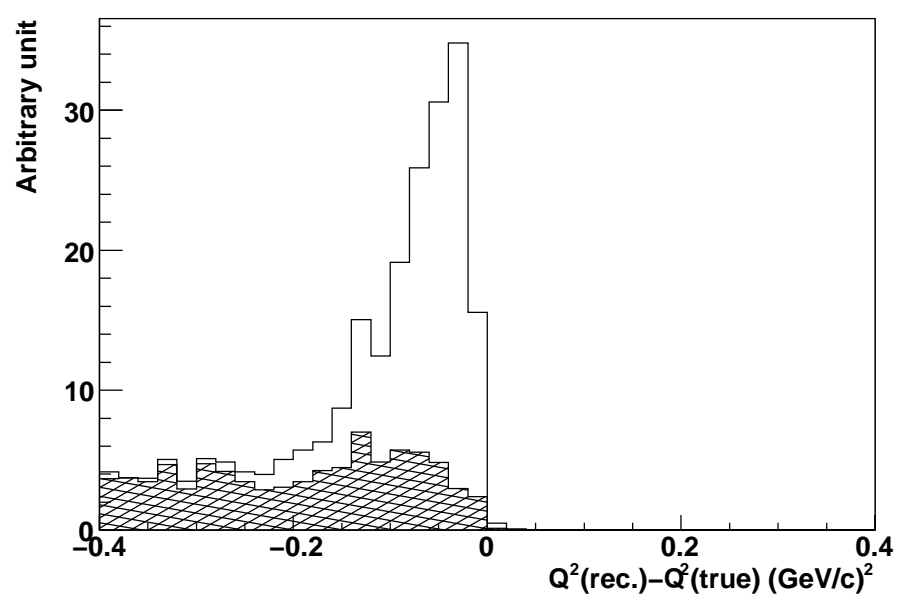

Figure 7.36: Resolution of reconstructed $Q^{2}$ for the $\mu+\pi$ events in the MRD penetrated sample after the pion track direction cut. The hatched histogram shows the background contribution.

background is comprised of $75 \%$ charged current DIS, and $25 \% \bar{\nu}_{\mu}$ events. The selection efficiency for the signal is estimated to be $3.1 \%$.

\section{6 $\sigma(\mathbf{C C}$ coherent $\pi) / \sigma(\mathrm{CC})$ cross section ratio}

We measure the cross section ratio of charged current coherent pion production] to total charged current interaction. 
Table 7.3: Event selection summary of MRD penetrated charged current coherent pion sample. The MC simulation is normalized to the number of the SciBar-MRD matched events in data.

\begin{tabular}{lrrrr}
\hline \hline Event selection & DATA & \multicolumn{2}{c}{ MC } & Coherent $\pi$ \\
& & Signal & B.G. & Efficiency \\
\hline Generated in SciBar fid.vol. & & 1,939 & 156,766 & $100 \%$ \\
SciBar-MRD matched & 30,337 & 978 & 29,359 & $50.4 \%$ \\
\hline MRD penetrated & 3,712 & 177 & 4,375 & $9.1 \%$ \\
2 track & 1,029 & 92 & 1,304 & $4.7 \%$ \\
Particle ID $(\mu+\pi)$ & 418 & 78 & 474 & $4.0 \%$ \\
Vertex activity cut & 167 & 71 & 186 & $3.6 \%$ \\
CC-QE rejection & 134 & 67 & 135 & $3.5 \%$ \\
Pion track direction cut & 107 & 66 & 109 & $3.4 \%$ \\
Reconstructed $Q^{2}$ cut & 57 & 60 & 40 & $3.1 \%$ \\
\hline \hline
\end{tabular}

\subsubsection{MRD stopped sample}

\section{Charged current coherent pion production}

The efficiency corrected number of charged current coherent pion events is calculated as

$$
N(\text { CC coherent } \pi)=\frac{N_{\text {obs }}-N_{\mathrm{BG}}}{\epsilon_{\text {coherent }}},
$$

where $N_{\text {obs }}$ is the number of observed events, $N_{\mathrm{BG}}$ is the number of background events estimated with the MC simulation, and $\epsilon_{\text {coherent }}$ is the selection efficiency of charged current coherent pion events calculated with the MC simulation, defined as

$$
\epsilon_{\text {coherent }}=\frac{\text { the number of selected CC coherent pion events }}{\text { the number of generated CC coherent pion events in the SciBar FV }} \text {. }
$$

After subtracting background and correcting for the selection efficiency, the number of charged current coherent pion candidates in the MRD-stopped sample is measured to be $179 \pm 190$ (stat); this error includes the uncertainty in the background estimation. No evidence of charged current coherent pion production is found in the sample. The neutrino energy dependence of the selection efficiency for charged current coherent pion events is shown in Fig. 7.37. The mean neutrino beam energy for true charged current coherent pion events in the sample is estimated to be $1.1 \mathrm{GeV}$ after accounting for the effects of the selection efficiency. The RMS of the neutrino beam energy is $0.27 \mathrm{GeV}$.

\section{Total charged current interaction}

The total number of charged current interactions is estimated by using the SciBarMRD matched sample. The number of charged current candidates after correcting for the selection efficiency is calculated as

$$
N(\mathrm{CC})=\frac{N_{\mathrm{obs}}^{\mathrm{CC}} \times p_{\mathrm{CC}}}{\epsilon_{\mathrm{CC}}},
$$



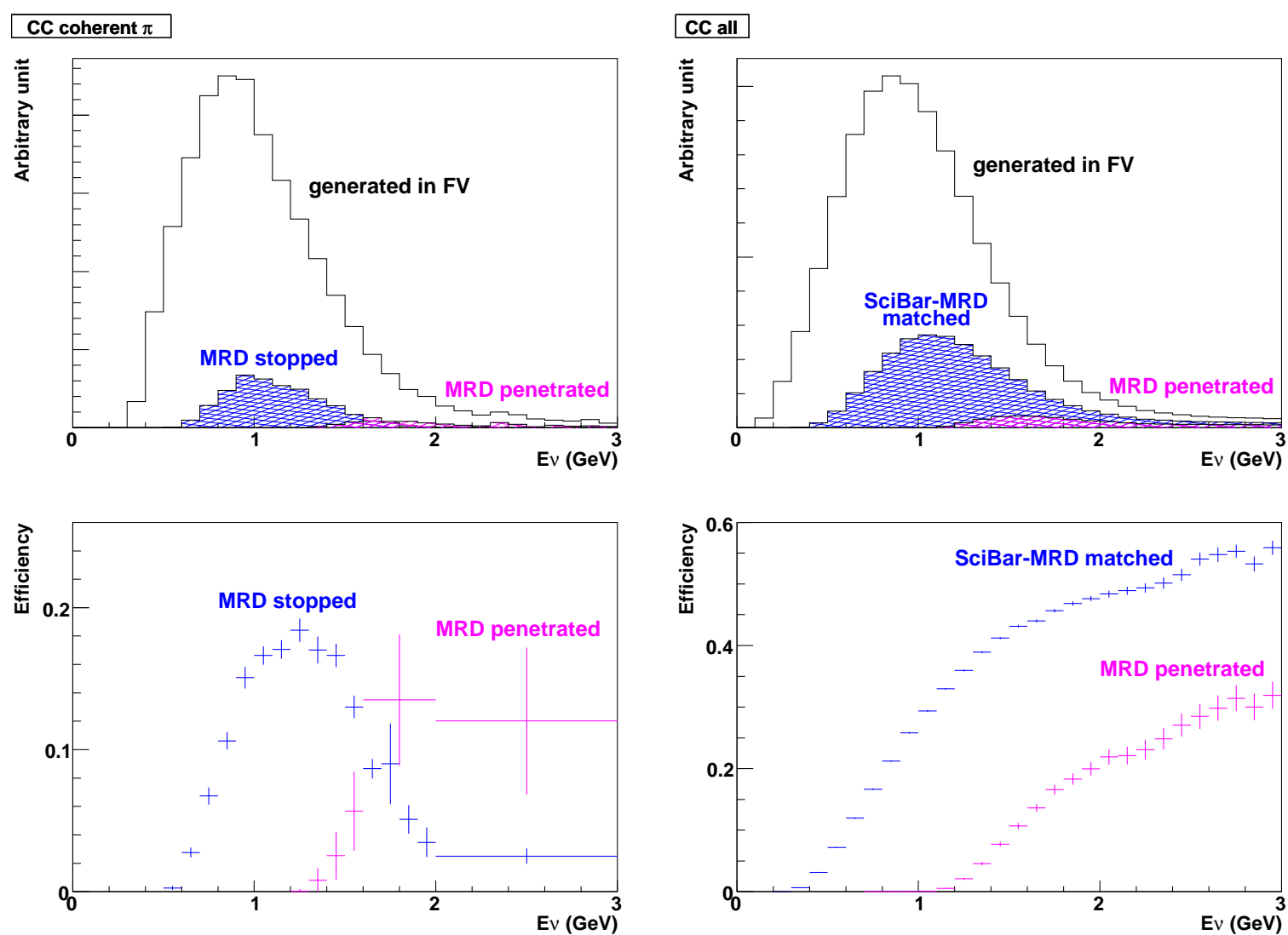

Figure 7.37: Neutrino energy spectra and selection efficiencies as a function of neutrino energy for charged current coherent pion events.

Figure 7.38: Neutrino energy spectra and selection efficiencies as a function of neutrino energy for all charged current events.

where $N_{\mathrm{obs}}^{\mathrm{CC}}$ is the number of observed charged current event candidates, $\epsilon_{\mathrm{CC}}$ and $p_{C C}$ are the selection efficiency and purity for charged current interactions in the sample, respectively.

As described in section 7.2.4, we observe 30,337 SciBar-MRD matched events. The selection efficiency and purity of charged current events are estimated to be $27.9 \%$ and $92.8 \%$, respectively. The neutrino energy dependence of the selection efficiency for charged current events is shown in Fig. 7.38. After correcting for the efficiency and purity, the number of charged current events is measured to be $(1.091 \pm 0.006($ stat $)) \times 10^{5}$.

\section{Cross section ratio}

Using this information, the ratio of the charged current coherent pion production to total charged current cross sections is measured to be

$$
\begin{aligned}
\frac{\sigma(\mathrm{CC} \text { coherent } \pi)}{\sigma(\mathrm{CC})} & =\frac{N(\mathrm{CC} \text { coherent } \pi)}{N(\mathrm{CC})} \\
& =\left(0.16 \pm 0.17(\text { stat. })_{-0.27}^{+0.30}(\text { sys. })\right) \times 10^{-2}
\end{aligned}
$$

at $1.1 \mathrm{GeV}$, where the systematic error is described later. The result is consistent with the non-existence of charged current coherent pion production, and hence we set an upper 
limit on the cross section ratio by using the likelihood distribution $(\mathcal{L})$ which is convolved with the systematic error. We calculate the $90 \%$ confidence level (C.L.) upper limit (UL) using the relation

$$
\frac{\int_{0}^{\mathrm{UL}} \mathcal{L} d x}{\int_{0}^{\infty} \mathcal{L} d x}=0.9 .
$$

Since the systematic error is asymmetric, we employ an asymmetric Gaussian as the likelihood distribution:

$$
\mathcal{L}=\frac{1}{\sqrt{2 \pi} \sigma}\left[\exp \left(-\frac{(x-m)^{2}}{2 \sigma_{+}{ }^{2}}\right) \theta(x-m)+\exp \left(-\frac{(x-m)^{2}}{2 \sigma_{-}{ }^{2}}\right)\{1-\theta(x-m)\}\right],
$$

where $m$ is the measured central value, $\sigma_{+}$and $\sigma_{-}$are the total errors for positive and negative directions, respectively, and $\sigma \equiv\left(\sigma_{+}+\sigma_{-}\right) / 2$. If $m>0$, the upper limit is expressed as

$$
\mathrm{UL}=m+\sqrt{2} \sigma_{+} \cdot \operatorname{erf}^{-1}\left[0.9-0.1 \cdot \frac{\sigma_{-}}{\sigma_{+}} \cdot \operatorname{erf}\left(\frac{m}{\sqrt{2} \sigma_{-}}\right)\right]
$$

where

$$
\operatorname{erf}(x) \equiv \frac{2}{\sqrt{\pi}} \int_{0}^{x} \exp \left(-t^{2}\right) d t
$$

Therefore, the $90 \%$ C.L. upper limit is

$$
\frac{\sigma(\mathrm{CC} \text { coherent } \pi)}{\sigma(\mathrm{CC})}<0.67 \times 10^{-2}
$$

at a mean neutrino energy of $1.1 \mathrm{GeV}$.

\subsubsection{MRD penetrated sample}

\section{Charged current coherent pion production}

After subtracting background and correcting for the selection efficiency, the number of charged current coherent pion candidates in the MRD penetrating sample is measured to be $548 \pm 254$ (stat). As in the MRD stopping sample, this includes the uncertainty due to the background estimation. The mean neutrino beam energy for true charged current coherent pion events in the sample is estimated to be $2.2 \mathrm{GeV}$ after accounting for the effects of the selection efficiency. The RMS of the neutrino beam energy is $0.80 \mathrm{GeV}$.

\section{Total charged current interaction}

Due to the higher neutrino energy in the charged current coherent pion sample, the MRD penetrated charged current sample is chosen to estimate the number of total charged current interactions at a similar neutrino energy. We observe 3,712 MRD penetrated events, and the efficiency and purity of true $\nu_{\mu}$ charged current events are estimated to be $4.5 \%$ and $97.5 \%$, respectively. The impurity largely comes from $\bar{\nu}_{\mu}$ charged current events. After correcting for the efficiency and purity, the number of charged current events is measured to be $(0.804 \pm 0.013$ (stat) $) \times 10^{5}$. A $26 \%$ difference between the MRD matched and penetrated samples is found. However, the difference can be explained by 
Table 7.4: Summary of the systematic errors in the charged current coherent pion cross section ratio.

\begin{tabular}{|c|c|c|c|c|}
\hline \multirow{2}{*}{$\begin{array}{l}\text { Source } \\
\text { Detector response }\end{array}$} & \multicolumn{2}{|c|}{$\begin{array}{l}\text { MRD stopped } \\
\text { error }\left(\times 10^{-2}\right)\end{array}$} & \multicolumn{2}{|c|}{$\begin{array}{l}\text { MRD penetrated } \\
\text { error }\left(\times 10^{-2}\right)\end{array}$} \\
\hline & +0.10 & -0.18 & +0.18 & -0.18 \\
\hline Nuclear effect & +0.20 & -0.07 & +0.19 & -0.09 \\
\hline Neutrino interaction model & +0.17 & -0.04 & +0.08 & -0.04 \\
\hline Neutrino beam & +0.07 & -0.11 & +0.27 & -0.13 \\
\hline Event selection & +0.07 & -0.14 & +0.06 & -0.05 \\
\hline Total & +0.30 & -0.27 & +0.39 & -0.25 \\
\hline
\end{tabular}

the uncertainty in the neutrino flux prediction within a $2 \sigma$ level, and this is expected to be a small effect to the cross section ratio measurement as long as we choose the charged current inclusive sample which has a similar mean neutrino energy to the corresponding coherent pion sample.

\section{Cross section ratio}

The ratio of the charged current coherent pion to total charged current production cross sections is measured to be

$$
\frac{\sigma(\mathrm{CC} \text { coherent } \pi)}{\sigma(\mathrm{CC})}=\left(0.68 \pm 0.32(\text { stat. })_{-0.25}^{+0.39}(\text { sys. })\right) \times 10^{-2}
$$

at $2.2 \mathrm{GeV}$. The systematic error is described later. No significant evidence for charged current coherent pion production is observed, and hence we set an upper limit on the cross section ratio at $90 \%$ C.L.:

$$
\frac{\sigma(\mathrm{CC} \text { coherent } \pi)}{\sigma(\mathrm{CC})}<1.36 \times 10^{-2}
$$

at a mean neutrino energy of $2.2 \mathrm{GeV}$.

\subsection{Systematic errors}

The sources of systematic error are divided into five categories, (i) detector response and track reconstruction, (ii) nuclear effects, (iii) neutrino interaction models, (iv) neutrino beam, and (v) event selection. We vary these sources within their uncertainties and take the resulting change in the cross section ratio as the systematic uncertainty of the measurement. Table 7.4 summarizes the uncertainties in the charged current coherent pion cross section ratio for the MRD stopped and MRD penetrated samples. The total systematic error is ${ }_{-0.27}^{+0.30} \times 10^{-2}$ for the MRD stopped sample, and ${ }_{-0.25}^{+0.39} \times 10^{-2}$ for the MRD penetrated sample. 
Table 7.5: Systematic errors in the charged current coherent pion cross section ratio due to the uncertainties in the detector responses.

\begin{tabular}{lcc|cc}
\hline \hline Source & $\begin{array}{c}\text { MRD stopped } \\
\text { error }\left(\times 10^{-2}\right)\end{array}$ & \multicolumn{2}{c}{ MRD penetrated } \\
& error $\left(\times 10^{-2}\right)$ \\
\hline Crosstalk & +0.04 & -0.05 & +0.12 & -0.04 \\
1 pe resolution & +0.05 & -0.02 & +0.07 & -0.06 \\
Scintillator quenching & +0.03 & -0.17 & +0.07 & -0.16 \\
Pion interaction in SciBar & +0.01 & -0.01 & +0.01 & -0.00 \\
Hit threshold & +0.07 & -0.03 & +0.09 & -0.02 \\
\hline Subtotal & +0.10 & -0.18 & +0.18 & -0.18 \\
\hline \hline
\end{tabular}

\subsubsection{Detector response and track reconstruction}

The uncertainties in the crosstalk and single photoelectron resolution of the MA-PMT, the scintillator quenching effect, simulation of pion interaction in SciBar, and the hit threshold for track reconstruction are considered. The systematic errors are summarized in Table 7.5.

\section{Crosstalk and 1 pe resolution of the MA-PMT}

The crosstalk of the MA-PMT was measured to be $3.15 \%$ for adjacent channels, with an absolute error of $0.4 \%$. The single photoelectron resolution of the MA-PMT is set to $50 \%$ in the simulation, and the absolute error is estimated to be $\pm 20 \%$. We prepare several MC event sets by changing the crosstalk level and single photoelectron resolution separately, and take the change of the charged current coherent pion cross section ratio as the systematic error.

\section{Scintillator quenching}

Birk's constant of the SciBar scintillator was measured to be $0.0208 \pm 0.0023 \mathrm{~cm} / \mathrm{MeV}[90]$ and is varied within the measurement error to evaluate the systematic error.

\section{Pion interaction in SciBar}

As mentioned in Section 5.6.2, a $10 \%$ difference of the total pion-carbon cross section between the GEANT4 simulation and external measurements is seen for higher energy pion. To evaluate systematic error due to this, we vary the cross section by $\pm 10 \%$, and take the change as the systematic error.

\section{Hit threshold}

The channel-by-channel variation of the photoelectron to energy conversion factor is measured to be approximately $20 \%$. Therefore, the hit threshold for track reconstruction is varied by $\pm 20 \%$ to evaluate the systematic error. 
Table 7.6: Systematic errors in the charged current coherent pion cross section ratio due to the uncertainties in the nuclear effects.

\begin{tabular}{lcc|cr}
\hline \hline Source & $\begin{array}{c}\text { MRD stopped } \\
\text { error }\left(\times 10^{-2}\right)\end{array}$ & $\begin{array}{c}\text { MRD penetrated } \\
\text { error }\left(\times 10^{-2}\right)\end{array}$ \\
\hline Pion absorption cross section & +0.00 & -0.05 & +0.11 & -0.00 \\
Pion inelastic cross section & +0.17 & -0.00 & +0.04 & -0.00 \\
Nucleon re-scattering cross section & +0.11 & -0.05 & +0.15 & -0.08 \\
Fermi momentum & +0.02 & -0.02 & +0.03 & -0.03 \\
\hline Subtotal & +0.20 & -0.07 & +0.19 & -0.09 \\
\hline \hline
\end{tabular}

\subsubsection{Nuclear effects}

We consider uncertainties in final state interactions inside the nucleus. This includes rescattering of nucleons and pions in the initial target nucleus. In addition, the uncertainty in the Fermi momentum of nucleons is considered. The systematic errors are summarized in Table 7.6.

\section{Pion interaction in the nucleus}

For pions produced by neutrino interactions, uncertainties on the cross sections for pion absorption and pion inelastic scattering in the nucleus are considered. The cross section of pion charge exchange is negligible compared with the other effects and is hence neglected. In the momentum range of pions from $\Delta$ decays, the cross section measurement uncertainty for both absorption and inelastic scattering is approximately $30 \%$ [85].

\section{Nucleon re-scattering}

Nucleon re-scattering in the nucleus affects vertex activity. The uncertainty in the cross section is estimated to be $10 \%$. We prepare MC event sets in which the nucleon rescattering cross section is changed by $\pm 10 \%$, and take the change of the charged current coherent pion cross section ratio as the systematic error.

\section{Fermi momentum}

In the NEUT simulation, the Fermi momentum of nucleons is set to $217 \mathrm{MeV} / c$ for carbon. According to electron quasi-elastic scattering data [108], the value is approximately $221 \pm 5 \mathrm{MeV} / c$. Therefore, an uncertainty of $\pm 5 \mathrm{MeV} / c$ is assigned. We estimate the systematic error due to this effect by eliminating events in which the momentum of the initial state nucleon is greater than $212 \mathrm{MeV} / c$. The change in the cross section ratio is negligible small compared to the other systematic error sources. We quote the same error for both sides.

\subsubsection{Neutrino interaction models}

The uncertainties in the axial vector mass for QE and resonant $\pi$, resonant $\mu^{-} n \pi^{+} / \mu^{-} p \pi^{+}$ cross section ratio, and the possible low $Q^{2}$ suppression of charged current resonant pion 
Table 7.7: Systematic errors in the charged current coherent pion cross section ratio due to the uncertainties in the neutrino interaction models.

\begin{tabular}{lcc|cc}
\hline \hline Source & $\begin{array}{c}\text { MRD stopped } \\
\text { error }\left(\times 10^{-2}\right)\end{array}$ & \multicolumn{2}{c}{ MRD penetrated } \\
error $\left(\times 10^{-2}\right)$
\end{tabular}

events are considered. The systematic errors are summarized in Table 7.7.

\section{Axial vector mass for $\mathrm{QE}$ and resonant $\pi$}

In the NEUT simulation, we set the axial vector mass $M_{A}$ to $1.21 \mathrm{GeV} / c^{2}$ for both $\mathrm{QE}$ and resonant pion production. The uncertainty in this value is estimated to be approximately $\pm 0.1 \mathrm{GeV} / c^{2}$ based on recent measurements [63,64]; results from past experiments are systematically lower than the recent measurements [109], and thus we only vary $M_{A}$ to $1.11 \mathrm{GeV} / c^{2}$, and take that change as the systematic error.

\section{Resonant $\mu^{-} n \pi^{+} / \mu^{-} p \pi^{+}$cross section ratio}

We consider the uncertainty in the charged current resonant $\mu^{-} n \pi^{+} / \mu^{-} p \pi^{+}$cross section ratio. This is motivated by the fact that resonant $\mu^{-} n \pi^{+}$channel is a dominant background in the final charged current coherent pion sample. We recall Equation 7.13 - Equation 7.16 which give us the expected number of events for each sample in the MC simulation:

$$
\begin{aligned}
& N_{i, 1 \text { trk }}^{\text {exp }}=R_{\text {norm }} \cdot\left[n_{i, 1 \text { trk }}^{\mathrm{QE}}+R_{\text {res }} n_{i, 1 \text { trk }}^{\text {res }}+R_{\text {other }} n_{i, 1 \text { trk }}^{\text {other }}\right] \\
& N_{i, \mu p}^{\text {exp }}=R_{\text {norm }} \cdot R_{2 \operatorname{trk} / 1 \text { trk }} \cdot R_{p / \pi} \cdot\left[n_{i, \mu p}^{\mathrm{QE}}+R_{\text {res }} n_{i, \mu p}^{\text {res }}+R_{\text {other }} n_{i, \mu p}^{\text {other }}\right] \\
& N_{i, \mu \pi \mathrm{H}}^{\exp }=R_{\text {norm }} \cdot R_{2 \operatorname{trk} / 1 \mathrm{trk}} \cdot\left[n_{i, \mu \pi \mathrm{H}}^{\mathrm{QE}}+R_{\mathrm{res}} n_{i, \mu \pi \mathrm{H}}^{\mathrm{res}}+R_{\mathrm{other}} n_{i, \mu \pi \mathrm{H}}^{\text {other }}\right] \\
& N_{i, \mu \pi \mathrm{L}}^{\exp }=R_{\text {norm }} \cdot R_{2 \operatorname{trk} / 1 \text { trk }} \cdot R_{\text {act }} \cdot\left[n_{i, \mu \pi \mathrm{L}}^{\mathrm{QE}}+R_{\text {res }} n_{i, \mu \pi \mathrm{L}}^{\text {res }}+R_{\text {other }} n_{i, \mu \pi \mathrm{L}}^{\text {other }}\right] .
\end{aligned}
$$

To evaluate the uncertainty in the charged current resonant $\mu^{-} n \pi^{+} / \mu^{-} p \pi^{+}$cross section ratio using SciBooNE data samples, we introduce the parameter $R_{\text {res-n }}$, and make the following replacement:

$$
R_{\text {res }} n_{i, j}^{\mathrm{res}} \rightarrow R_{\mathrm{res}}\left(n_{i, j}^{\mathrm{res}-\mathrm{p}}+R_{\mathrm{res}-\mathrm{n}} n_{i, j}^{\mathrm{res}-\mathrm{n}}\right),
$$

where $n_{i, j}^{\text {res-p }}$ and $n_{i, j}^{\text {res-n }}$ are the number of resonant $\mu^{-} p \pi^{+}$and $\mu^{-} n \pi^{+}$events in each bin in each sub-sample, respectively. The parameter $R_{\text {res-n }}$ effectively changes the resonant $\mu^{-} n \pi^{+} / \mu^{-} p \pi^{+}$cross section ratio. Since the total resonant pion production cross section is already constrained using SciBooNE data samples as described in Section 7.4, we fit data samples by changing only the parameter $R_{\text {res-n }}$, while the other systematic parameters are fixed to the best fit values. We obtain the result $R_{\text {res }-\mathrm{n}}=1.02 \pm 0.07$, and thus the uncertainty in the resonant $\mu^{-} n \pi^{+} / \mu^{-} p \pi^{+}$ratio is estimated to be $7 \%$. 


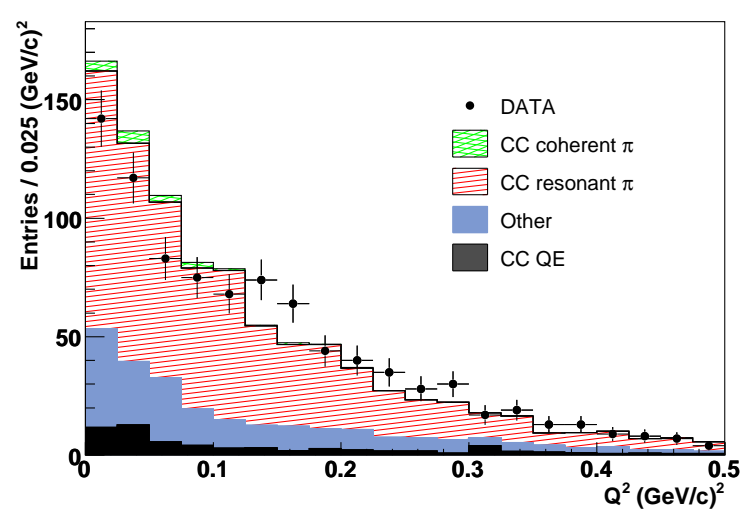

Figure 7.39: Reconstructed $Q^{2}$ for $\mu+\pi$ events with vertex activity after CC-QE rejection and pion track direction cut.

\section{Low $Q^{2}$ suppression of resonant pion events}

In addition, a disagreement of the $Q^{2}$ shape is observed in the $\mu+\pi$ events with vertex activity where charged current resonant pion production is dominant, as shown in Fig. 7.29. We estimate the systematic uncertainty, due to evident low $Q^{2}$ suppression of charged current resonant pion production even after the MC tuning, by re-weighting the true $Q^{2}$ distribution of charged current resonant pion events according to the observed low $Q^{2}$ deficit.

To make the re-weighting factor as a function of true $Q^{2}$, we reconstruct $Q^{2}$ by assuming that the out-going hadron is a $\Delta(1232)$ instead of a proton. Figure 7.40 shows the reconstructed $Q^{2}$ assuming $\Delta(1232)$, the expected $Q^{2}$ resolution estimated with the MC simulation, and the DATA/MC ratio as a function of reconstructed $Q^{2}$ for the sample. We fit the DATA/MC ratio by linear function indicated in the figure, and use it as the re-weighting function.

\subsubsection{Neutrino beam}

The uncertainties in the secondary particle production cross sections in proton-beryllium interactions, hadronic interactions in the target or horn, and the horn magnetic field model are considered as described in Section 3.2.4. Uncertainties associated with the delivery of the primary proton beam to the beryllium target and the primary beam optics, which result in the overall normalization uncertainty, are not considered in this analysis since it cancels in the cross section ratio.

The change in the neutrino beam spectrum due to these uncertainties is calculated by drawing random parameter vectors and weighting each event by a factor corresponding to the variation of the yield of the parent meson with the given momentum and angle. The extraction of the charged current coherent pion cross section ratio is repeated for each beam systematic parameter vector; The efficiency for charged current coherent pion events, the efficiency and purity for the charged current inclusive samples, and the number of background events are calculated using each parameter vector. The number of observed events is obtained from data.

Figure 7.41 shows the distribution of the charged current coherent pion cross section ratio obtained by using 1,000 neutrino flux predictions. The mean and sigma of the distribution are $0.14 \times 10^{-2}$ and $0.09 \times 10^{-2}$, respectively. Since the result using the default 

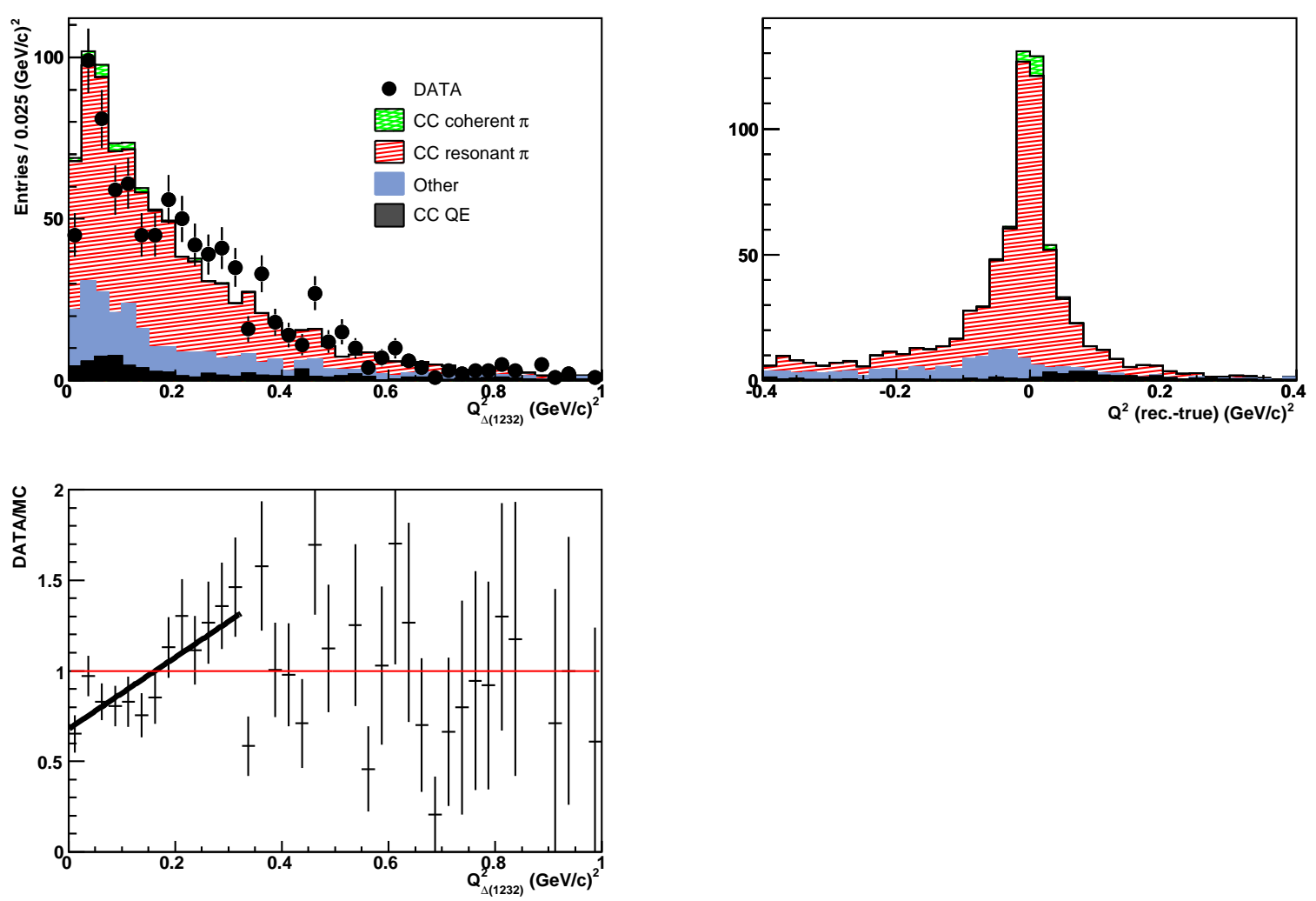

Figure 7.40: Reconstructed $Q^{2}$ assuming $\Delta(1232)$, expected $Q^{2}$ resolution, and DATA/MC ratio as a function of reconstructed $Q^{2}$ for $\mu+\pi$ events with vertex activity after CC-QE rejection and pion track direction cut.

MC simulation is $0.16 \times 10^{-2}$, we assign the systematic error of $(+0.07,-0.11) \times 10^{-2}$. The same is done for the MRD penetrated sample, and we assign the systematic error of $(+0.27,-0.13) \times 10^{-2}$.

\subsubsection{Event selection}

For the event selection variable $\Delta \theta_{p}$, we evaluate the systematic uncertainty in the cross section ratio by varying the cut placement. The uncertainty in the $\Delta \theta_{p}$ cut for charged current quasi-elastic rejection is estimated to be \pm 5 degrees. For the other variables, we already tuned the MC simulation using the migration parameters or considered possible systematic sources. Therefore, we do not include additional systematic uncertainties due to these selections.

\subsection{Summary and discussion}

Having not observed evidence for charged current coherent pion production, we set $90 \%$ confidence level upper limits on the cross section ratio of charged current coherent 


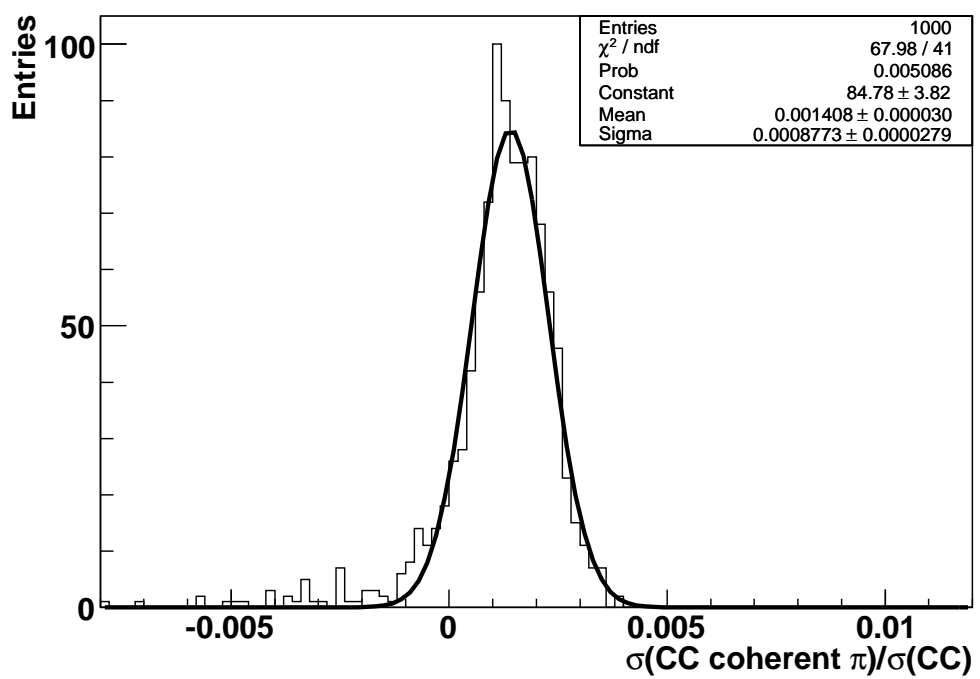

Figure 7.41: The variation of the charged current coherent pion cross section ratio for the MRD stopped sample using 1,000 neutrino flux predictions within the systematic uncertainty.

pion production to the total charged current cross section;

$$
\frac{\sigma(\mathrm{CC} \text { coherent } \pi)}{\sigma(\mathrm{CC})}<0.67 \times 10^{-2}
$$

at mean neutrino energy $1.1 \mathrm{GeV}$ and

$$
\frac{\sigma(\mathrm{CC} \text { coherent } \pi)}{\sigma(\mathrm{CC})}<1.36 \times 10^{-2}
$$

at mean neutrino energy $2.2 \mathrm{GeV}$. According to the Rein-Sehgal model [31,34] implemented in our simulation, the cross section ratio is expected to be $2.04 \times 10^{-2}$ for both cases. Our limits correspond to $33 \%$ and $67 \%$ of the prediction at $1.1 \mathrm{GeV}$ and $2.2 \mathrm{GeV}$, respectively. Our results are consistent with the $\mathrm{K} 2 \mathrm{~K}$ result; $\sigma(\mathrm{CC}$ coherent $\pi) / \sigma(\mathrm{CC})<$ $0.60 \times 10^{-2}$ at $90 \%$ C.L. measured in a $1.3 \mathrm{GeV}$ wide-band neutrino beam. ${ }^{1}$ We reveal that the Rein-Sehgal model widely used in neutrino oscillation experiments breaks down at the neutrino energy region of a few $\mathrm{GeV}$. The results have been published in Ref. [110].

Figure 7.42 shows the comparison of our results with theoretical predictions. Our 90\% CL upper limits on the ratio of charged current coherent pion production to total charged current cross sections are converted to the upper limits on the absolute cross section by using total charged current cross section predicted by the NEUT simulation; the total charged current cross section averaged over the MRD stopped and MRD penetrated samples are $1.05 \times 10^{-38} \mathrm{~cm}^{2} /$ nucleon and $1.76 \times 10^{-38} \mathrm{~cm}^{2} /$ nucleon, respectively, estimated with the MC simulation. As shown in the figure, several recent models predict a considerably smaller coherent cross section, which appears consistent with our results. However, these models have not been validated existing experimental results at higher neutrino energy.

\footnotetext{
${ }^{1}$ The $90 \%$ C.L. upper limit is calculated as UL $=m+1.28 \sigma$ which is different from our definition.
} 


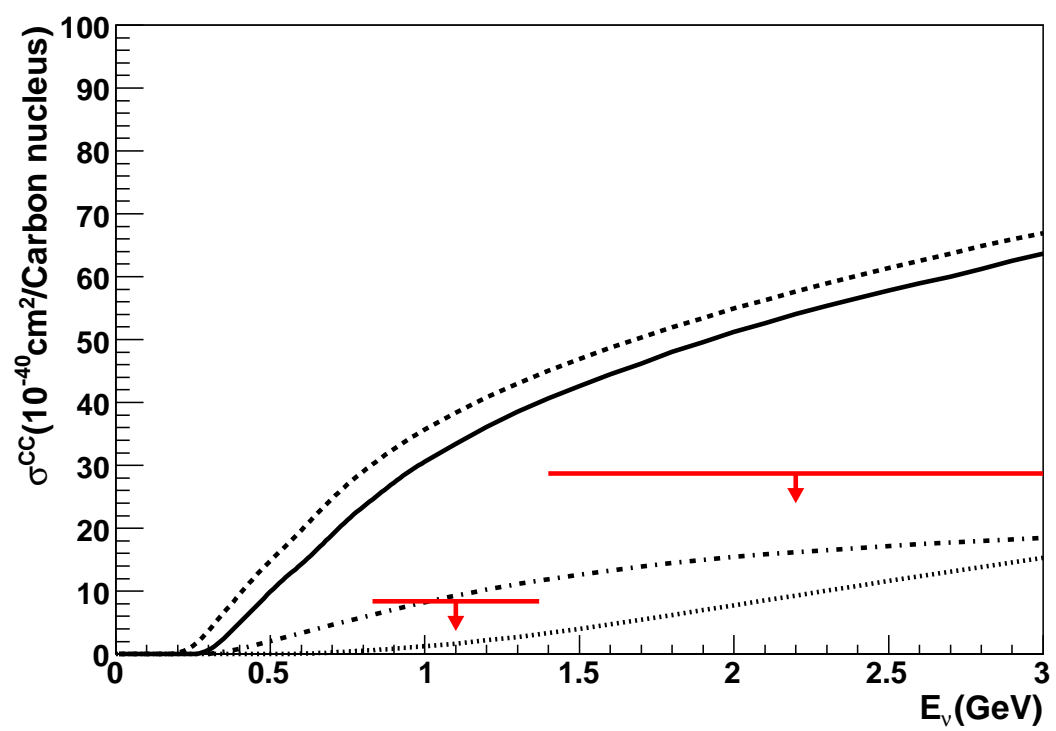

Figure 7.42: Comparison of our results with theoretical predictions. The red lines with arrows indicate our 90\% upper limits. The limits are obtained from our 90\% CL upper limits on the ratio of charged current coherent pion production to total charged current cross sections and total charged current cross section predicted by the NEUT simulation. The Rein and Sehgal model with lepton mass effects (solid line), the Rein and Sehgal model without lepton mass effects (dashed), the model of Kartavtsev et al. (dotted), and the model of Alvarez-Ruso et al. (dashed-dotted) are shown.

After we published our results, some investigations have been already performed from the phenomenological point of view $[111,112]$. Recently, Berger and Sehgal point out that the Rein-Sehgal model overestimates the pion-Carbon interaction cross sections at the pion energy region of resonance $\left(0.2<p_{\pi}(\mathrm{GeV} / c)<0.4\right)[112]$. They propose to use the cross sections obtained from pion-Carbon scattering experiments instead of using the scaling model of pion-nucleus cross sections. Their predictions on the cross section ratio of charged current coherent pion production to the total charged current cross section are $0.58 \times 10^{-2}$ at neutrino energy of $1.1 \mathrm{GeV}$ and $0.68 \times 10^{-2}$ at neutrino energy of $2.2 \mathrm{GeV}$, and both are consistent with our results. From the fact that the original Rein-Sehgal prediction for pion-Carbon scattering overlaps with this empirical result in the region $p_{\pi}>0.7 \mathrm{GeV} / c$, this modification is expected to affect only neutrino energy below $2 \mathrm{GeV}$ where the pion-Carbon cross section in the resonance region largely contributes to the calculation. Therefore, the Rein-Sehgal model would explain plenty of experimental data on coherent pion production in the wide neutrino energy range with a small modification.

On the other hand, neutral current coherent pion production is observed by the MiniBooNE experiment in the similar neutrino energy range. The fraction of neutral current coherent pion production to all neutral current single neutral pion production is measured to be $(19.5 \pm 1.1$ (stat) \pm 2.5 (sys) $\%$, which is $65 \%$ of the Rein-Sehgal prediction, at mean neutrino energy of $1.2 \mathrm{GeV}$. It is still difficult to explain both our upper limits on charged current coherent pion production and MiniBooNE's positive result on the neutral current channel at the same time. In the MiniBooNE neutral current coherent pion measurement, 
the signal is two photons from $\pi^{0}$ decay, and thus the observable kinematic variables which can be used to extract coherent pion events are the $\pi^{0}$ momentum and angle. Meanwhile, in the charged current coherent pion measurement in the SciBooNE experiment, there are plenty of kinematic variables: for example, muon momentum and angle, and the length and angle of the second track. Therefore, SciBooNE can proceed with a further investigation of the result. The future prospects of coherent pion production measurements in SciBooNE are discussed in Chapter 8.

In addition, the neutrino energy dependence of charged current coherent pion production in the neutrino energy range up to $20 \mathrm{GeV}$ will be also addressed by the Minerva experiment [113] in the near future. 


\section{Chapter 8}

\section{Discussion on Future Prospects}

In this chapter, we discuss future prospects of charged current single charged pion production measurements in the SciBooNE experiment. Since these analyses are ongoing, preliminary results presented here are not official SciBooNE results yet.

\subsection{Coherent pion production}

\subsubsection{Further study of neutrino coherent pion production}

As discussed in Section 7.8, Berger and Sehgal published a new prediction on coherent pion production after we published our result on a search for charged current coherent pion production. Interestingly, their prediction is just below our upper limit at neutrino energy $1.1 \mathrm{GeV}$, and this inspires us to a further investigation of the neutrino data to check compatibility of our data with their prediction.

Figure 8.1 shows the distributions of muon momentum, angle, pion track length, and pion angle for the MRD stopped coherent pion sample. The distributions of data in Figure 8.1 are basically in agreement with the background, but the data excess seems to cluster in a certain kinematic region: $p_{\mu}>0.7 \mathrm{GeV} / c, \theta_{\mu}<15$ degrees, $L_{\pi}<50 \mathrm{~cm}$, and $\theta_{\pi}<30$ degrees. Since the data excess appears only at the high muon momentum region, the sample is further divided into two sub-samples: $p_{\mu}<0.7 \mathrm{GeV} / c$ and $p_{\mu}>0.7 \mathrm{GeV} / c$. Figure 8.2 shows the distributions of muon angle, pion track length, and pion angle for these two different muon momentum regions in the MRD stopped coherent pion sample. In the lower muon momentum sample, all the kinematic distributions reasonably agree with the background shape. However, in the higher muon momentum sample, the shape of the data distributions are different from those of the background distributions, and thus the data excess cannot be explained by the normalization of the background.

In the case of charged current coherent pion production, muon and pion are expected to be emitted back-to-back in the $x-y$ plane because of the conservation of momentum. Therefore, the kinematic variable called $\Delta \phi$ is defined as shown in Figure 8.3. The coherent pion events are expected to distribute around $\Delta \phi=0$. Figure 8.4 shows the $\Delta \phi$ distributions for two different muon momentum regions in the MRD stopped coherent pion sample. In the lower muon momentum sample, the data and MC distributions agree well. The charged current quasi-elastic events also distribute around $\Delta \phi=0$ because of two-body interaction. On the other hand, the data excess is found around $\Delta \phi=0$ in the higher muon momentum sample. With all distributions considered, charged current 

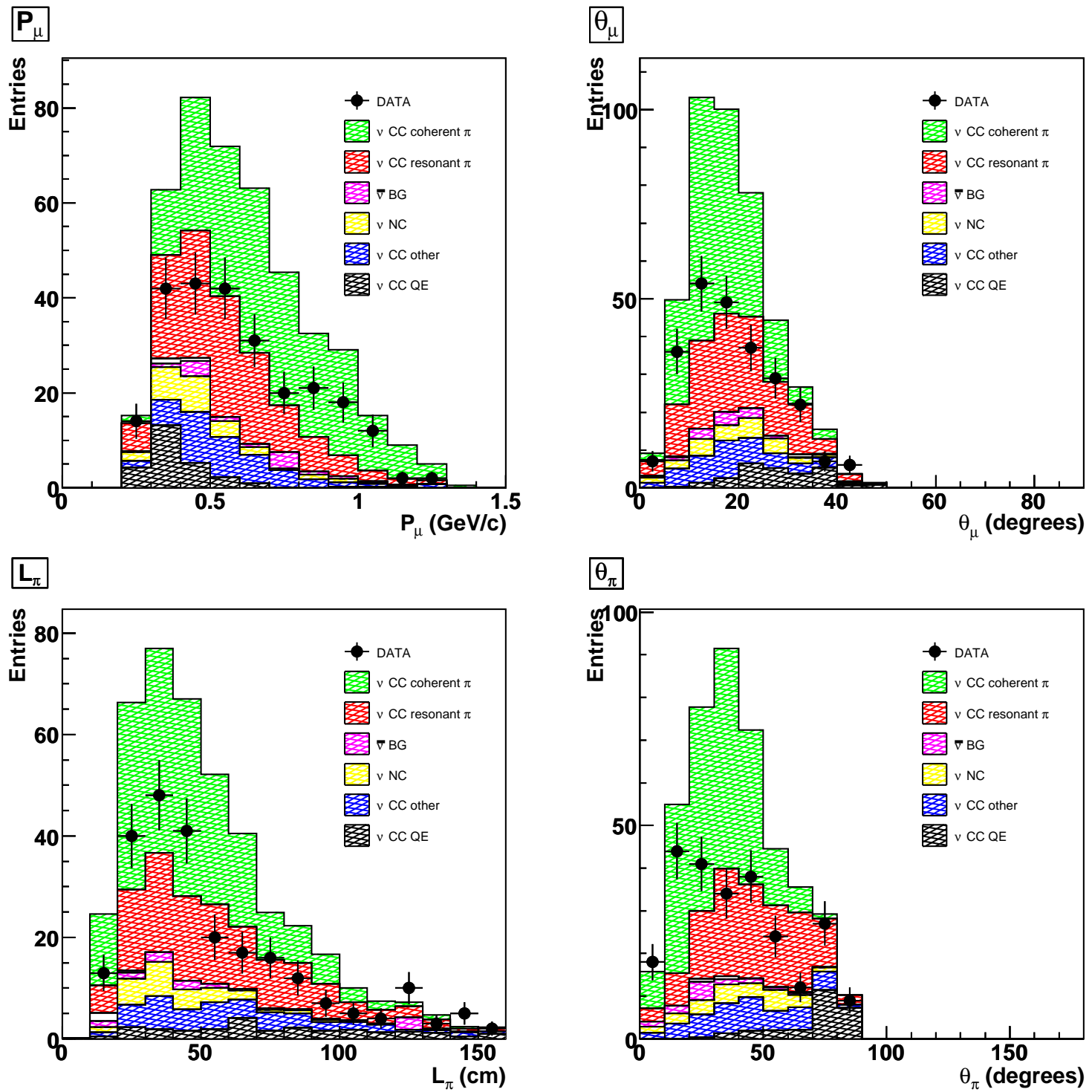

Figure 8.1: Muon momentum, angle, pion track length, and pion angle distributions for the MRD stopped coherent pion sample.

coherent pion production starts to appear in the higher muon momentum (and thus higher neutrino energy) sample. The significance of the observed signal above the background is almost $4 \sigma$ as far as the statistical error is concerned. Although systematic error is not included yet, there is a potential of reporting a first positive result on charged current coherent pion production in a few-GeV neutrino energy region.

Figure 8.6 shows distributions of pion momentum and angle for charged current coherent pion production based on the Rein-Sehgal model, and the selection efficiencies for the MRD stopped and penetrated coherent pion samples estimated with the MC simulation. In this analysis, we rely on the Rein-Sehgal model for the prediction of the kinematic distributions for charged current coherent pion production. However, these efficiency curves will be helpful when we compare data with the other theoretical models in the future. 

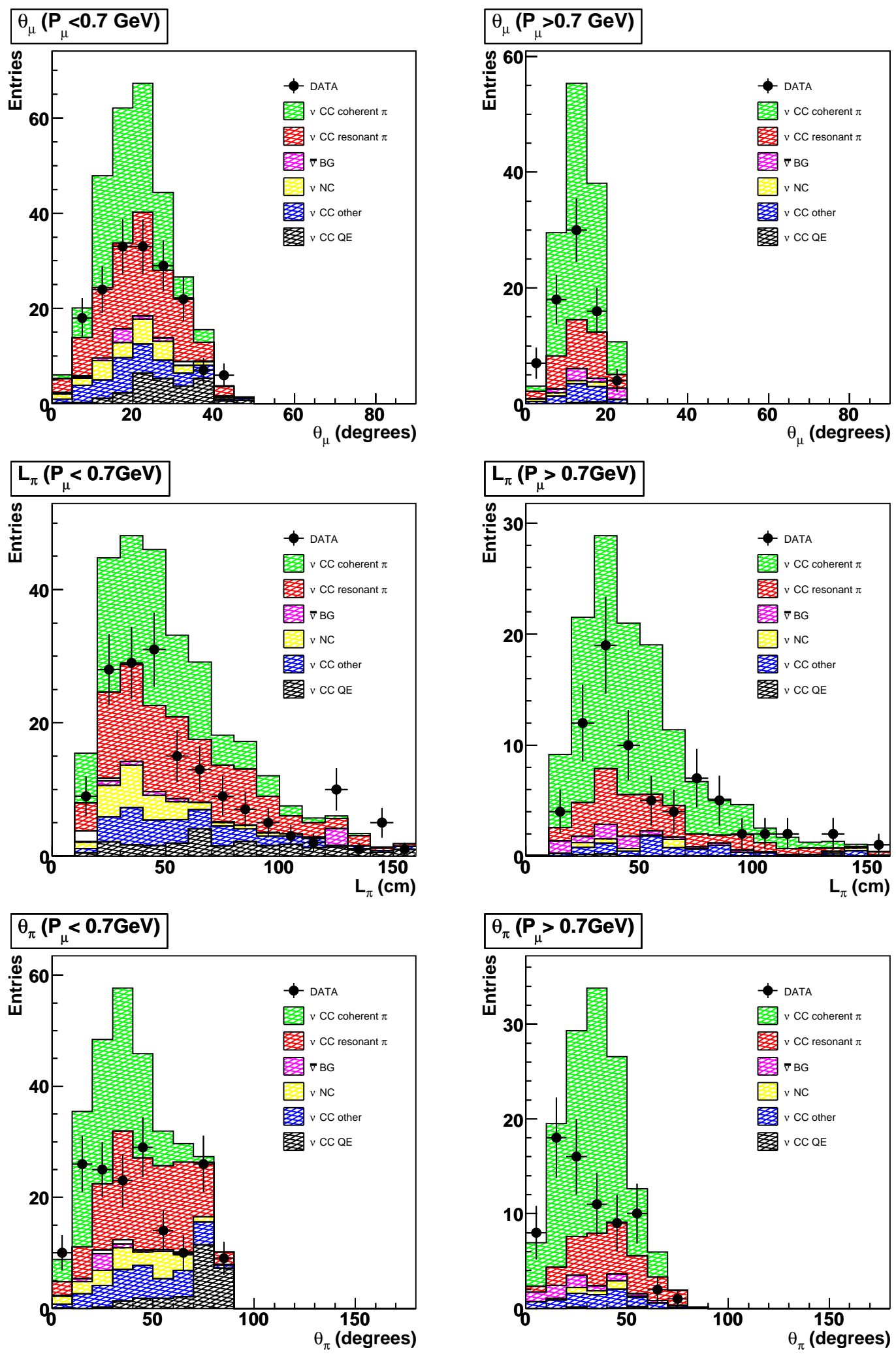

Figure 8.2: Muon angle, pion track length, and pion angle distributions for two different muon momentum regions in the MRD stopped coherent pion sample. 


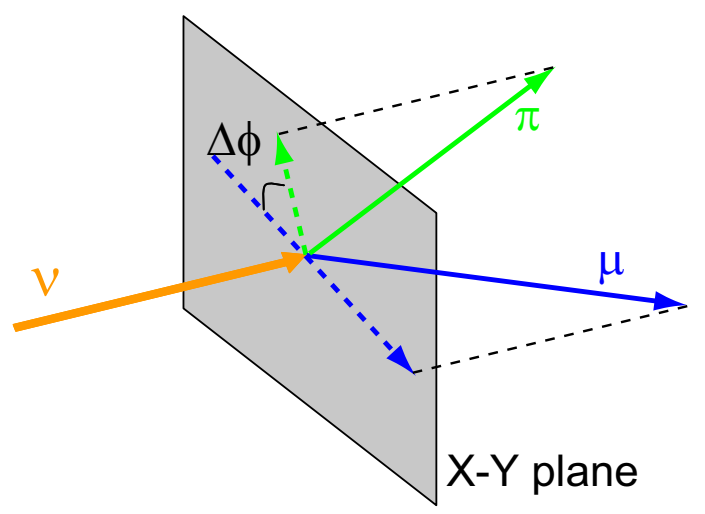

Figure 8.3: Definition of the kinematic variable $\Delta \phi$.
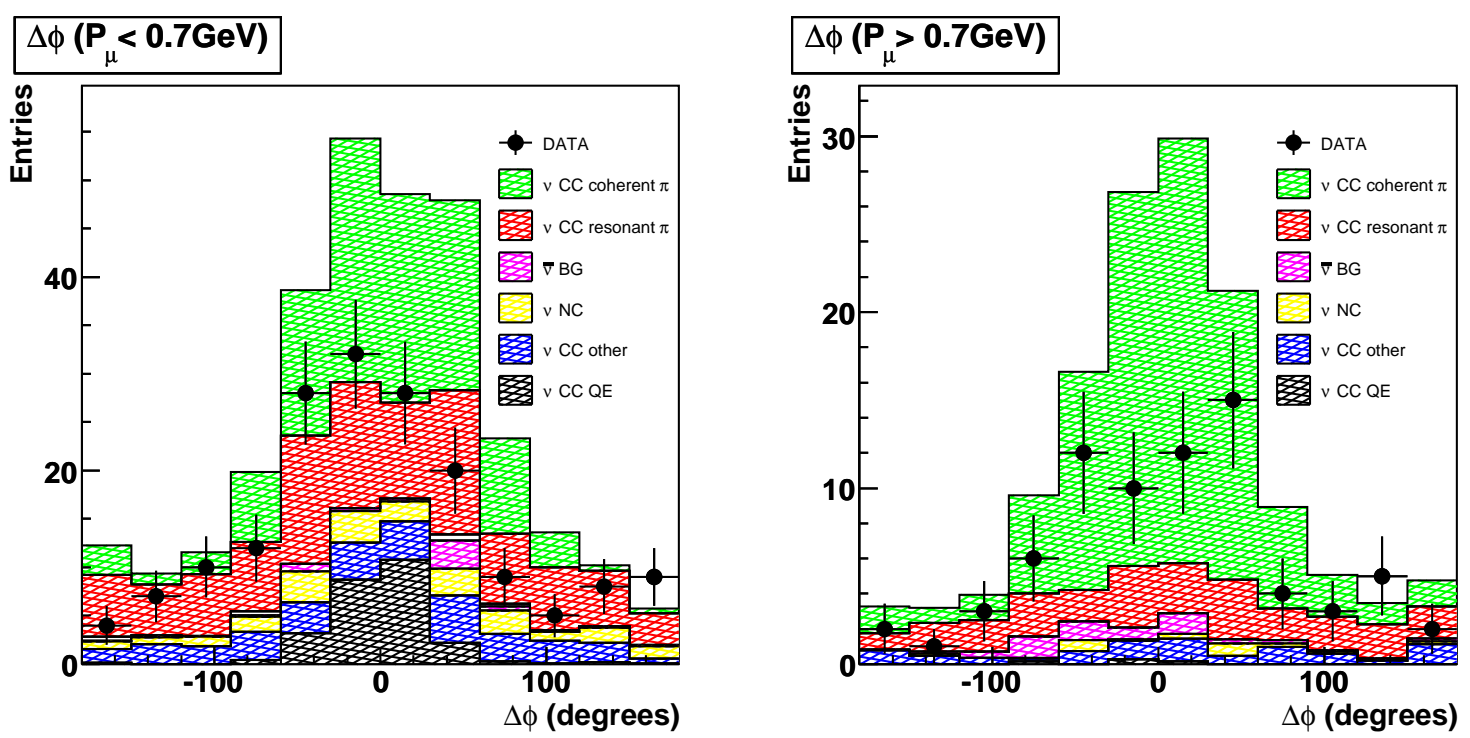

Figure 8.4: $\Delta \phi$ distributions for two different muon momentum regions in the MRD stopped coherent pion sample. 

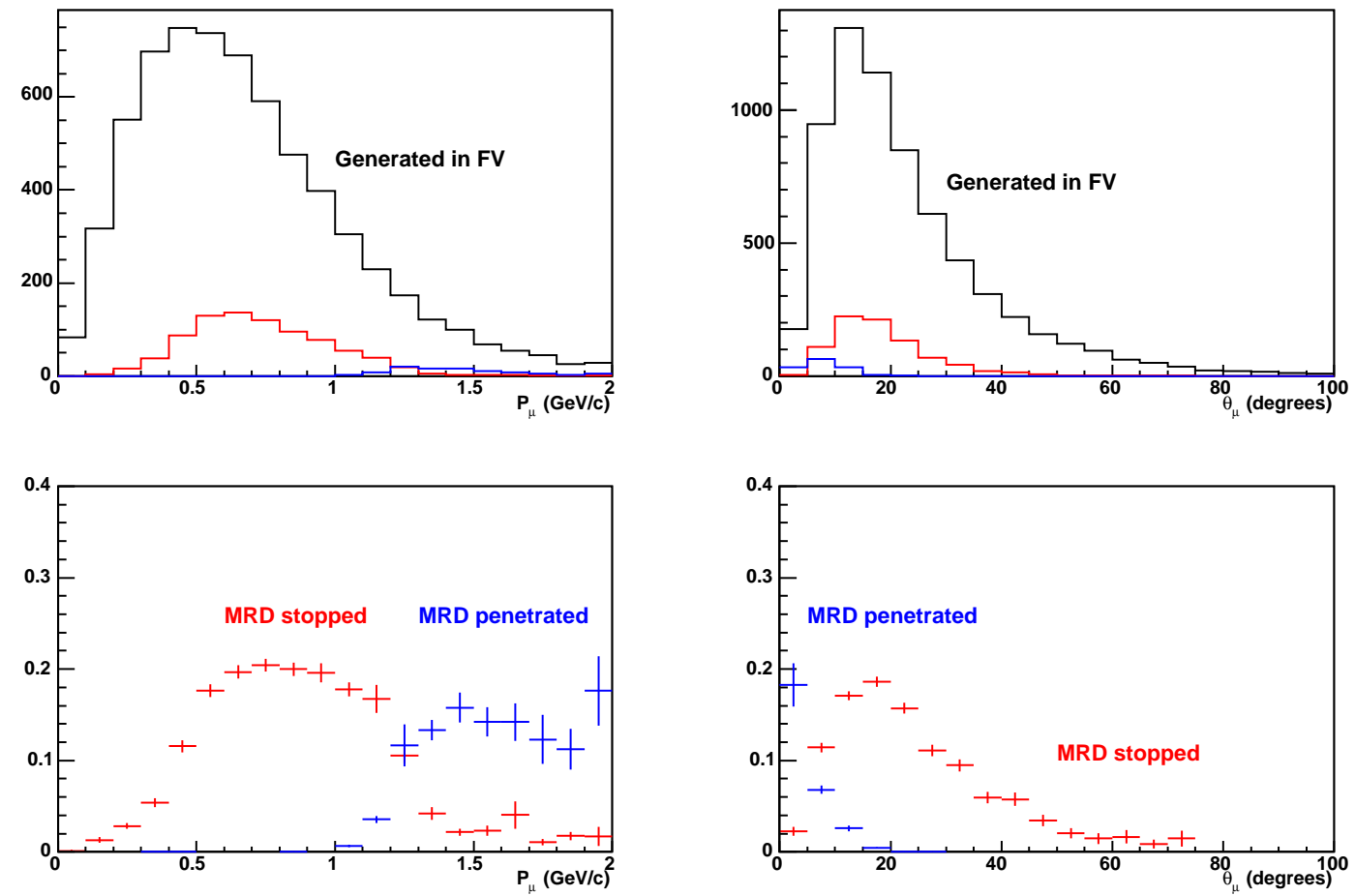

Figure 8.5: Distributions of muon momentum and angle for charged current coherent pion production based on the Rein-Sehgal model, and the selection efficiencies for the MRD stopped and penetrated coherent pion samples estimated with the MC simulation.
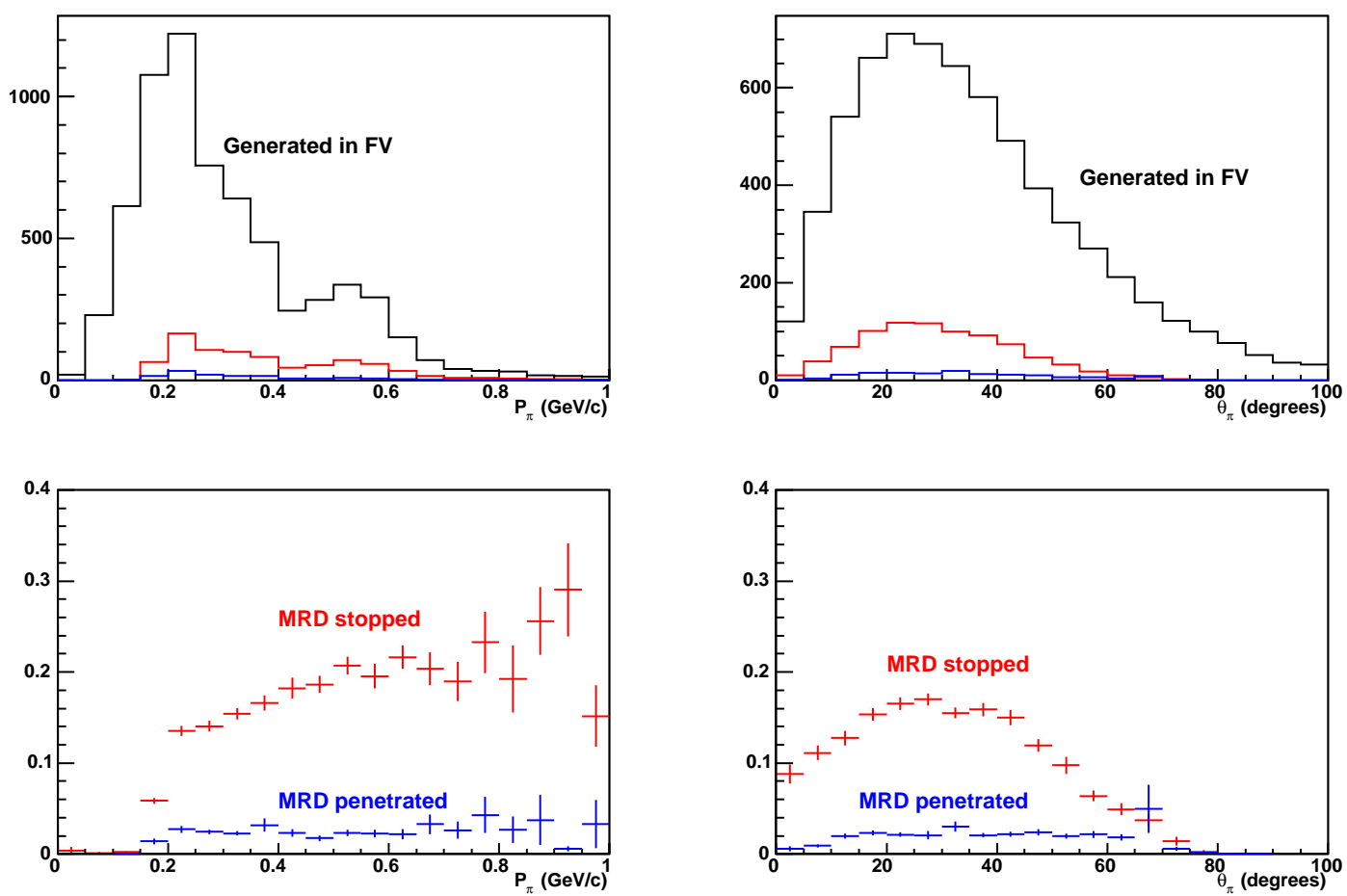

Figure 8.6: Distributions of pion momentum and angle for charged current coherent pion production based on the Rein-Sehgal model, and the selection efficiencies for the MRD stopped and penetrated coherent pion samples estimated with the MC simulation. 


\subsubsection{Antineutrino coherent pion production}

Most models predict similar absolute cross sections for neutrino and antineutrino coherent pion production, which means the ratio of charged current coherent pion events to charged current inclusive events is expected to be larger in antineutrino data because of the reduced total $\bar{\nu}$ charged current cross section. Because of this, it will be interesting to repeat this analysis on SciBooNE's already collected antineutrino data.

We have started looking at the antineutrino data with the same selection criteria as applied to neutrino data. Here, we present a preliminary result on antineutrino charged current coherent pion production analyzing the antineutrino data corresponding to $1.01 \times$ $10^{20}$ POT (66\% of the total antineutrino data), compare it with the result from the neutrino data, and discuss future prospects.

Figure 8.7 shows the reconstructed $Q^{2}$ distributions for the one track events, $\mu+p$ events, $\mu+\pi$ events with activity and without activity in the MRD stopped sample. For the MC distributions, we use the default MC simulation described in Chapter 4; i.e. the charged current coherent pion prediction is based on the Rein-Sehgal model for both neutrinos and antineutrinos, and systematic parameters described in Section 7.4 are not introduced. The MC distributions are normalized to the number of the SciBar-MRD matched events in antineutrino data. Although the $\mathrm{MC}$ tuning for constraining systematic uncertainties is not performed yet, the data and $\mathrm{MC}$ distributions agree. In antineutrino beam, neutrino backgrounds (referred to as wrong-sign backgrounds) comprise $35 \%$ of the total event rate, and therefore it is crucial to measure the amount of the wrong-sign background. The $\mu+p$ sample can give a constraint on the wrong-sign background yield since this sample is dominated by events which are induced by wrong-sign neutrinos as shown in Figure 8.7 (b).

Figure 8.8 shows the reconstructed $Q^{2}$ distribution for the MRD stopped coherent pion sample before the $Q^{2}$ cut. In the signal region, $Q^{2}<0.1(\mathrm{GeV} / c)^{2}, 97$ events are observed, while the expected number of background events is 65. After subtracting background, the number of the signal candidates, the sum of $\bar{\nu}_{\mu}$ and $\nu_{\mu}$ coherent pion productions, is $32 \pm 10$ (stat). Based on the Rein-Sehgal model, 107 coherent pion events are expected in the signal region (92 $\bar{\nu}_{\mu}$ coherent pion events and $15 \nu_{\mu}$ coherent pion events). The amount of the observed signal corresponds to $30 \%$ of the Rein-Sehgal prediction, which is consistent with the upper limit obtained from the neutrino data. This is also consistent with the recent model prediction of Berger and Sehgal.

The significance of the observed signal above the background is more than $3 \sigma$ as far as the statistical error is concerned. Although systematic error is not included yet, with a full antineutrino data set, there is a potential of reporting a first positive result on antineutrino charged current coherent pion production in a few-GeV neutrino energy region.

\subsubsection{Neutral current coherent pion production}

Theoretical models also make concrete connections between the charged and neutral current coherent pion production processes. However, as discussed in Section 7.8, it is difficult to explain our null results on charged current coherent pion production and MiniBooNE's positive result on the neutral current channel at the same time. The SciBooNE neutral current coherent pion search is, therefore, also interesting and may shed consid- 

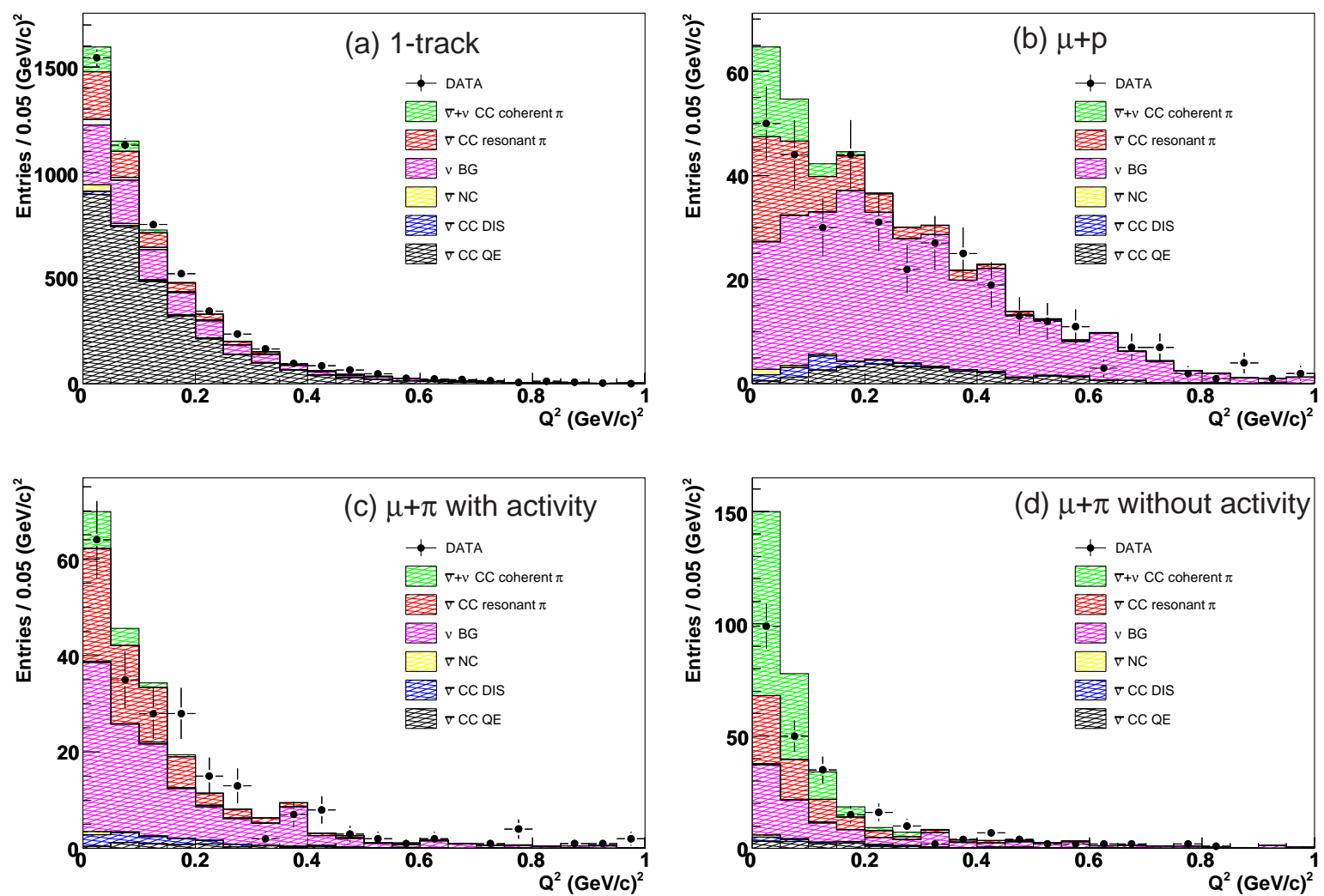

Figure 8.7: Reconstructed $Q^{2}$ for (a) the one track events, (b) $\mu+p$ events, (c) $\mu+\pi$ events with activity, and (d) $\mu+\pi$ events without activity. The MC tuning is not performed yet.

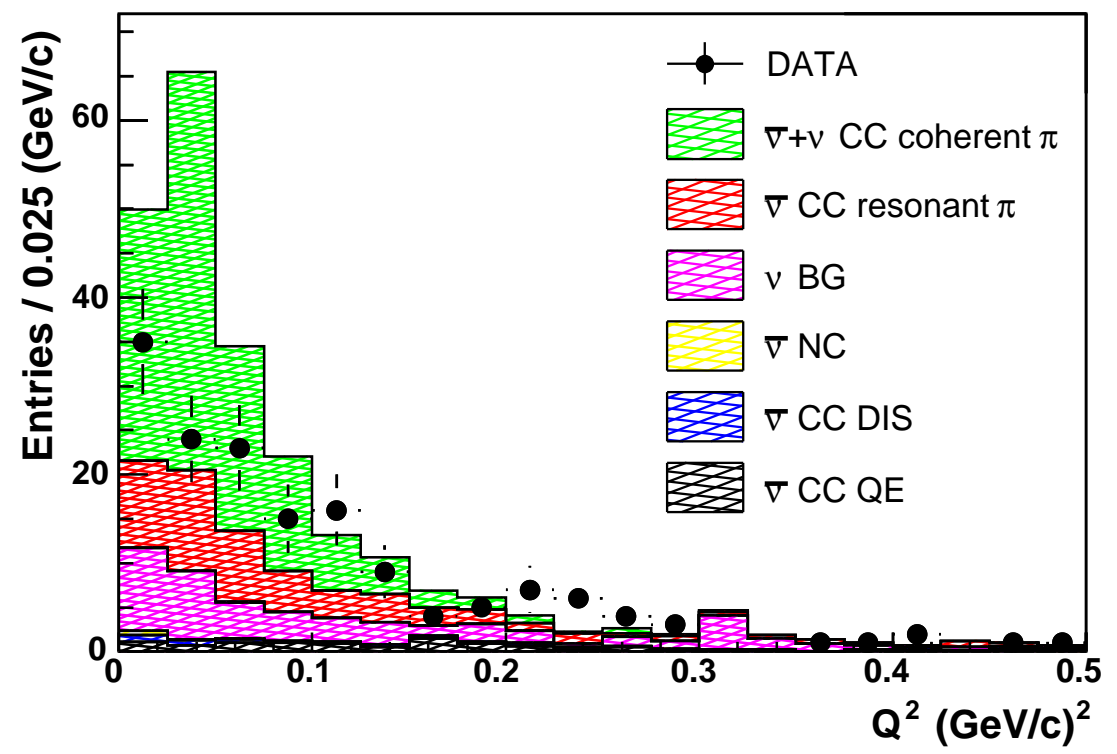

Figure 8.8: Reconstructed $Q^{2}$ for the $\mu+\pi$ events without vertex activity in the MRD stopped sample after the $\Delta \theta_{p}$ and pion track direction cuts. The MC tuning is not performed yet. 
erable light on the behavior of this interaction process. An analysis of neutral current neutral pion production in SciBooNE is currently in process [114].

\subsection{Resonant pion production}

Having finished a study of charged current coherent pion production, we start a measurement of cross sections of resonant pion productions: $\nu_{\mu} p \rightarrow \mu^{-} p \pi^{+}$and $\nu_{\mu} n \rightarrow \mu^{-} n \pi^{+}$, separately. The experimental signature of charged current resonant pion production in SciBooNE is the existence of two minimum ionizing particles which emerges from the common interaction vertex: a muon and a charged pion. Therefore, the signal candidates are basically extracted from the $\mu+\pi$ event sample described in Section 7.3. In order to separate $\nu_{\mu} p \rightarrow \mu^{-} p \pi^{+}$from $\nu_{\mu} n \rightarrow \mu^{-} n \pi^{+}$, the vertex activity is the good variable. In order to reduce charged current quasi-elastic events in these samples, the $\Delta \theta_{p}$ cut is imposed.

Figure 8.9 shows the muon momentum and angle distributions for the $\mu+\pi$ events with vertex activity in the MRD stopped sample after the $\Delta \theta_{p}$ cut. For the MC simulation, charged current coherent pion production is set to zero, based on our null result. The sample is dominated by charged current resonant $\mu^{-} p \pi^{+}$events, and thus this sample is used to extract the cross section of the resonant $\mu^{-} p \pi^{+}$channel. The largest background for this channel is charged current deep inelastic scattering in which multiple pions are produced.

On the other hand, Figure 8.10 shows the same distributions for the $\mu+\pi$ events without vertex activity in the MRD stopped sample after the $\Delta \theta_{p}$ cut. Although the fraction of charged current resonant $\mu^{-} n \pi^{+}$events is increased by the vertex activity cut, there still exists a large amount of background from charged current deep inelastic and resonant $\mu^{-} p \pi^{+}$interactions. Therefore, it might be difficult to extract the cross section of the resonant $\mu^{-} n \pi^{+}$channel, but this sample can be used to constrain the deep inelastic scattering and $\mu^{-} n \pi^{+}$cross sections for the $\mu^{-} p \pi^{+}$cross section measurement.

When we measure the resonant pion cross section, one of the largest source of the systematic uncertainty is the nuclear effects, especially pion absorption. In SciBooNE, if pion is absorbed in the nucleus, the resonant pion event is reconstructed as a one track event or a $\mu+p$ event. According to the MC simulation, approximately $40 \%$ of the resonant pion events in the one track sample or in the $\mu+p$ sample are such events. Figure 8.11 and Figure 8.12 show the muon momentum and angle distributions for the one track events and the $\mu+p$ events in the MRD stopped sample, respectively. Although these samples are dominated by charged current quasi-elastic interactions, the one track and $\mu+p$ samples can be used to constrain the strength of pion absorption in the nucleus.

Table 8.1 summarizes event samples for the resonant pion cross section measurement. SciBooNE has approximately 21,000 events which can be used the resonant pion measurement, and of these events, approximately 4,500 events are resonant $\mu^{-} p \pi^{+}$events. This is twice larger statistics than that used for the charged current resonant pion production measurement by the K2K Collaboration. With this enough statistics and better constraints on the nuclear effects, an energy dependent cross section measurement with a precision of $5-10 \%$ would be achievable. 

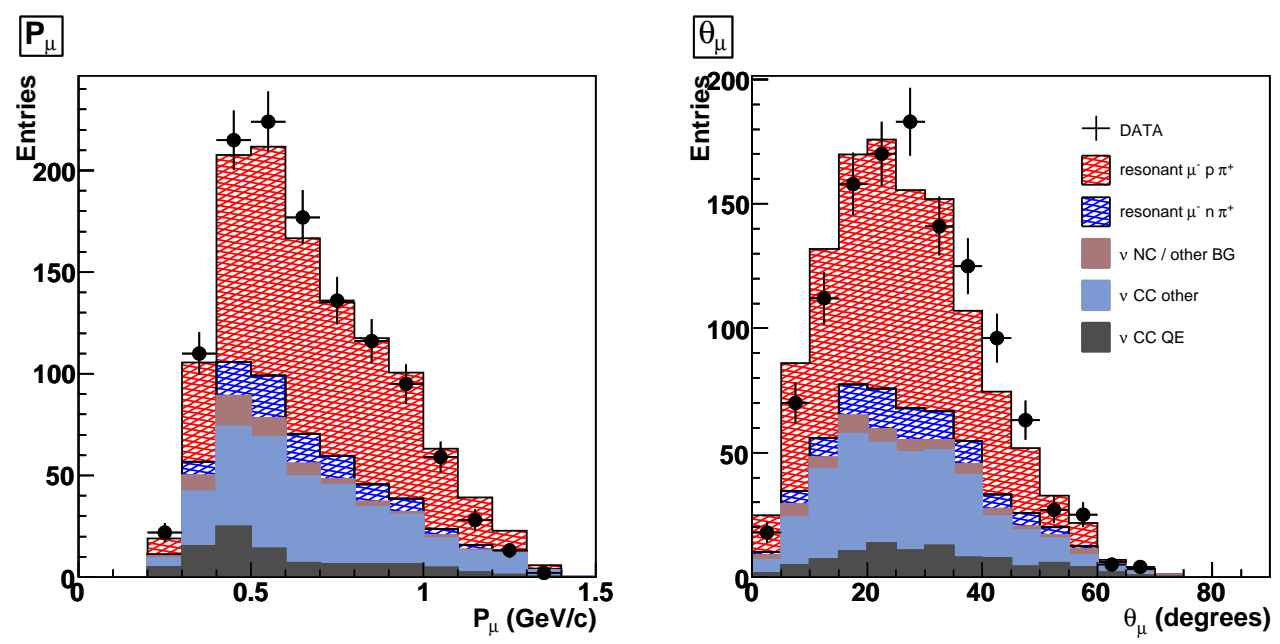

Figure 8.9: Muon momentum and angle for the $\mu+\pi$ events with vertex activity in the MRD stopped sample after the $\Delta \theta_{p}$ cut.
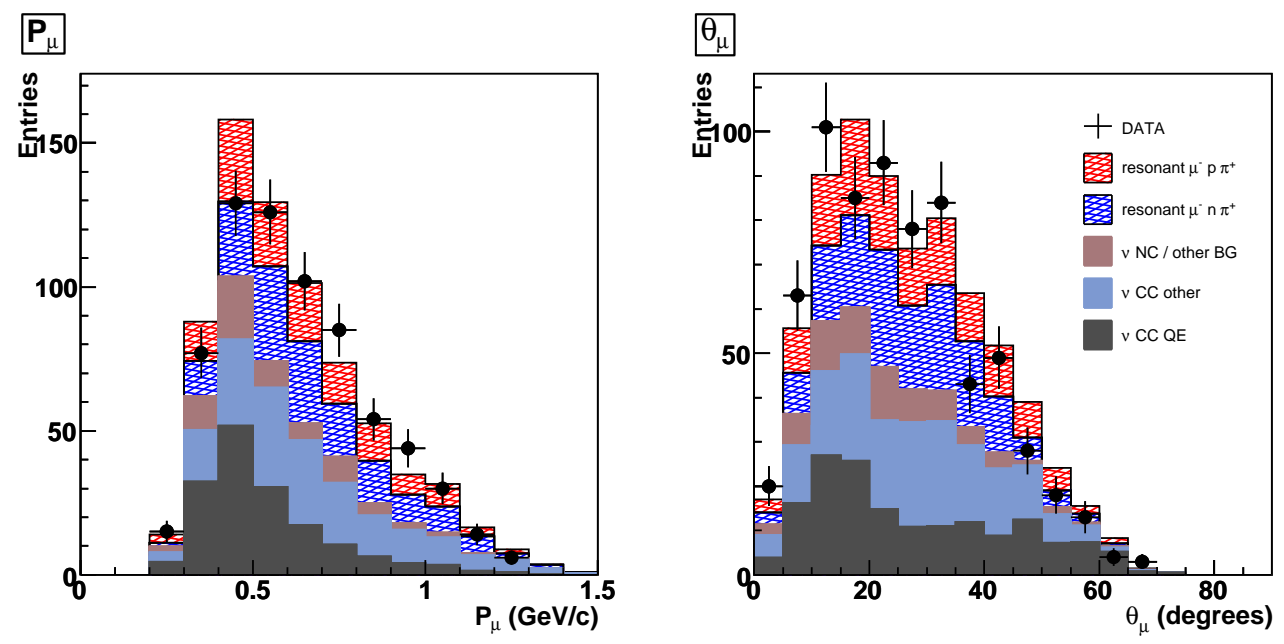

Figure 8.10: Muon momentum and angle for the $\mu+\pi$ events without vertex activity in the MRD stopped sample after the $\Delta \theta_{p}$ cut.

Table 8.1: Summary of event samples used for the resonant pion cross section measurement.

\begin{tabular}{lrrcccc}
\hline \hline Sample & DATA & \multicolumn{4}{c}{ MC } \\
& & $\mu^{-} p \pi^{+}$ & $\mu^{-} n \pi^{+}$ & CC-QE & CC-other & NC/Other \\
\hline 1 track & 15,387 & $17 \%$ & $5 \%$ & $55 \%$ & $11 \%$ & $12 \%$ \\
$\mu+p$ & 3,359 & $29 \%$ & $2 \%$ & $54 \%$ & $14 \%$ & $1 \%$ \\
$\mu+\pi$, w/ activity, nonQE & 1,197 & $55 \%$ & $8 \%$ & $8 \%$ & $26 \%$ & $3 \%$ \\
$\mu+\pi$, no activity, nonQE & 682 & $19 \%$ & $23 \%$ & $23 \%$ & $26 \%$ & $9 \%$ \\
\hline \hline
\end{tabular}



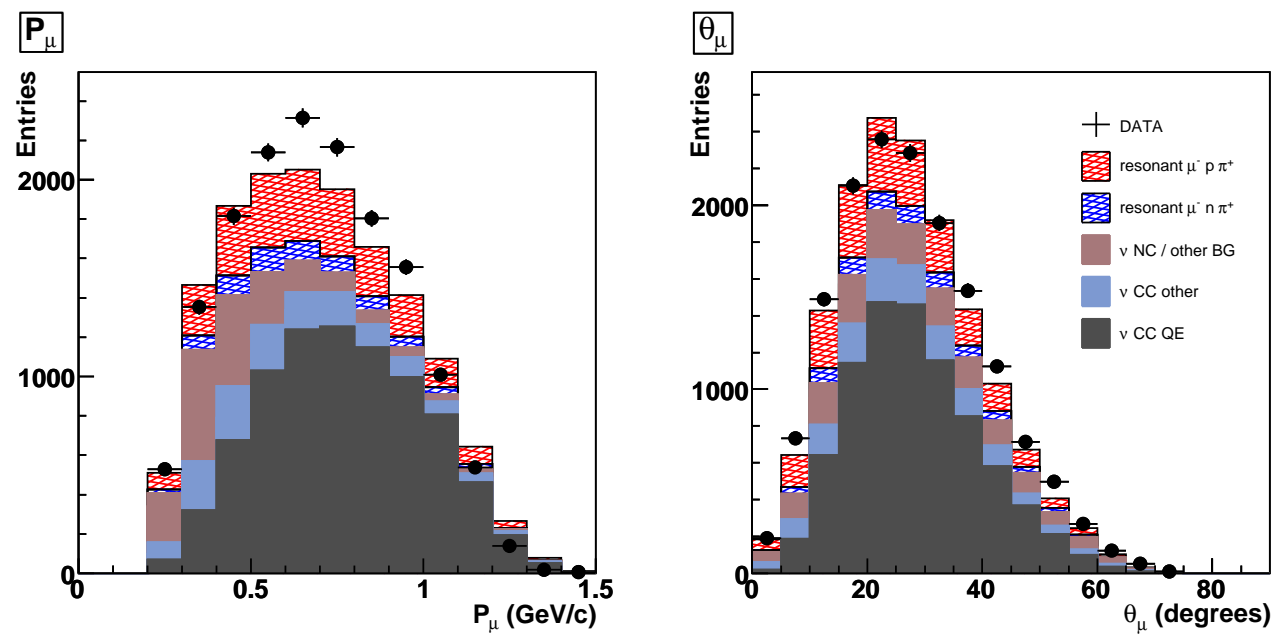

Figure 8.11: Muon momentum and angle for the one track events in the MRD stopped sample.
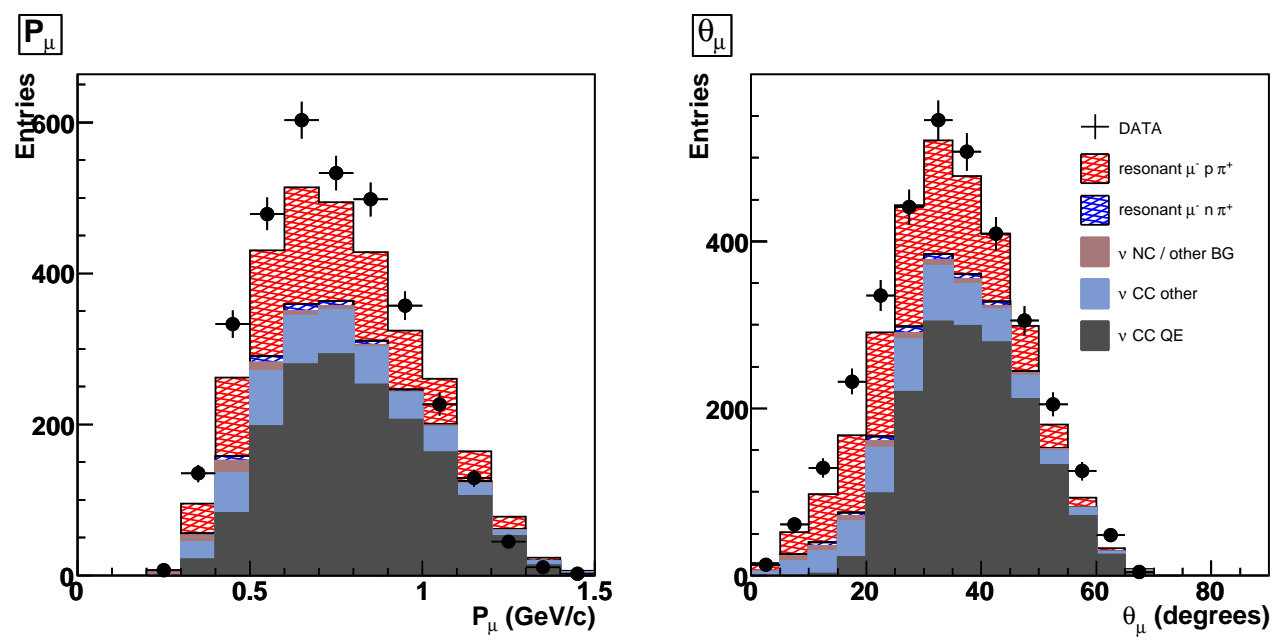

Figure 8.12: Muon momentum and angle for the $\mu+p$ events in the MRD stopped sample. 


\section{Chapter 9}

\section{Conclusions}

Understanding single charged pion production via neutrino-nucleus charged current interaction in the neutrino energy region of a few $\mathrm{GeV}$ is essential for future neutrino oscillation experiments since this process is a dominant background for $\nu_{\mu}$ disappearance measurements. There are two contributions to this process: single pion production via baryonic resonance $\left(\nu_{\mu} N \rightarrow \mu^{-} N \pi^{+}\right)$and coherent pion production interacting with the entire nucleus $\left(\nu_{\mu} A \rightarrow \mu^{-} A \pi^{+}\right)$, where $N$ is nucleon in the nucleus and $A$ is the nucleus.

The purpose of the study presented in this thesis is a precise measurement of charged current single charged pion productions, resonant and coherent pion productions, with a good final state separation in the neutrino energy region of a few $\mathrm{GeV}$. The SciBooNE experiment is designed for measuring neutrino-nucleus cross sections in these neutrino energies. We took data from June 2007 until August 2008, in both the neutrino and antineutrino beam. In total, $2.52 \times 10^{20}$ protons on target have been collected.

In this thesis, we focused on a study of charged current coherent pion production in the SciBooNE experiment. This is motivated by the fact that the contribution of coherent pion production in the region of small muon scattering angle is not small, and without measuring this component first, the precise determination of resonant pion production cross section can not be achieved. Furthermore, the coherent process is particularly interesting because it is deeply rooted in fundamental physics via Adler's partially conserved axial-vector current theorem. Using SciBooNE's full neutrino data set, corresponding to $0.99 \times 10^{20}$ protons on target, we have performed a search for charged current coherent pion production from muon neutrinos scattering on carbon, $\nu_{\mu}{ }^{12} \mathrm{C} \rightarrow \mu^{-12} \mathrm{C} \pi^{+}$with two distinct data samples. No evidence for coherent pion production is observed. We set $90 \%$ confidence level upper limits on the cross section ratio of charged current coherent pion production to the total charged current cross section;

$$
\frac{\sigma(\mathrm{CC} \text { coherent } \pi)}{\sigma(\mathrm{CC})}<0.67 \times 10^{-2}
$$

at mean neutrino energy $1.1 \mathrm{GeV}$ and

$$
\frac{\sigma(\mathrm{CC} \text { coherent } \pi)}{\sigma(\mathrm{CC})}<1.36 \times 10^{-2}
$$

at mean neutrino energy $2.2 \mathrm{GeV}$. The upper limits correspond to $33 \%$ and $67 \%$ of the Rein and Sehgal model prediction at $1.1 \mathrm{GeV}$ and $2.2 \mathrm{GeV}$, respectively. Our results are consistent with the result by the K2K experiment. We reveal that the Rein-Sehgal model 
widely used in neutrino oscillation experiments breaks down at the neutrino energy region of a few $\mathrm{GeV}$. This creates active controversies on the model of coherent pion production, and the understanding of coherent pion production is being progressed.

In addition, we have discussed future prospects of charged current single charged pion production measurements in SciBooNE. We have started a further investigation of neutrino charged current coherent pion production in response to a recent model prediction of Berger and Sehgal. Studies of charged current coherent pion production by antineutrinos and charged current resonant pion production have been started. An energy dependent cross section measurement of charged current resonant pion production with a precision of $5-10 \%$ would be achievable. 


\section{Appendix A}

\section{Track Reconstruction}

In this chapter, we describe hit preparation for tracking, track reconstruction in SciBar, and matching between the SciBar and MRD tracks.

\section{A.1 Hit preparation in SciBar}

\section{A.1.1 Crosstalk correction}

Due to the crosstalk on the photocathode of the MA-PMTs, there sometimes exists hits around channels that a charged particle actually passes through, and thus these hits make the track fuzzy. In order to remove such hits, a cross talk correction is applied to both the data and $\mathrm{MC}$ events before track reconstruction.

The matrix $M$ is the $64 \times 64$ crosstalk matrix, where the element $M_{i j}$ represents the fraction of channel $j$ 's signal that migrates to channel $i$ due to crosstalk. If $Q_{i}$ is the charge in channel $i$ before crosstalk, and $Q_{i}^{\prime}$ is the charge in channel $i$ after crosstalk, then

$$
Q_{i}^{\prime}=\sum_{j} M_{i j} Q_{j}
$$

The crosstalk correction is just the inverse process:

$$
Q_{i}=\sum_{j} M_{i j}^{-1} Q_{j}^{\prime}
$$

The crosstalk matrix is determined based on the measurement taken in laboratory. Figure A.1 illustrates the crosstalk effect. We consider a $5 \times 5$ channel array around the central channel where the light is injected. $Q_{0}$ is the total charge corresponds to the injected light, $Q$ is the observed charge in the central channel after crosstalk, and $a, b, \ldots, e$ are the ratios of the observed charge in a given channel to that in the central channel, measured in laboratory: $a=3.15 \%, b=0.7 \%, c=0.3 \%, d=0.2 \%$, and $e=0.1 \%$, averaged over the channels whose distance from the central channel are same. The crosstalk to farther channels is neglected. Thus, the total charge is calculated as

$$
Q_{0}=(1+4 a+4 b+4 c+8 d+4 e) Q
$$




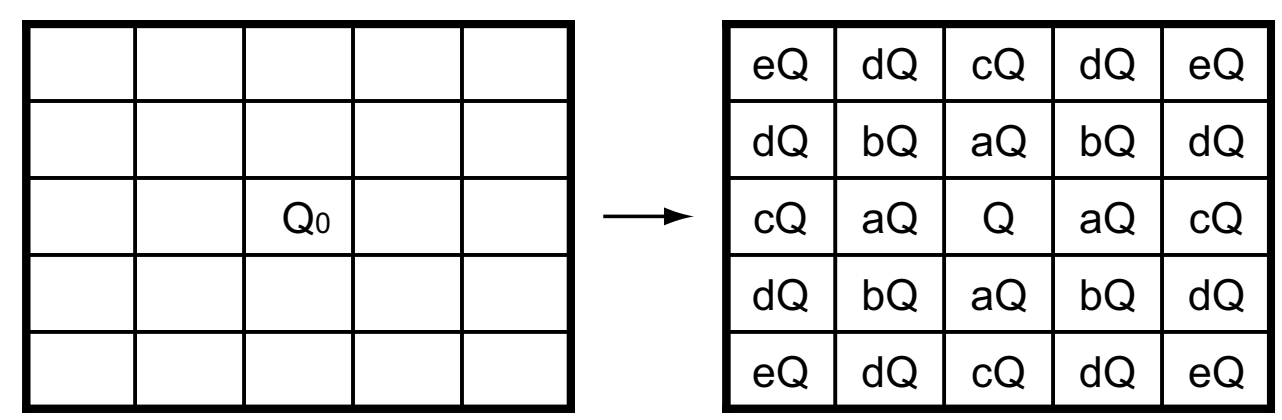

Figure A.1: Illustration of the crosstalk effect. $Q_{0}$ is the total charge corresponds to the injected light, $Q$ is the observed charge in the central channel after crosstalk, and $a, b, \ldots, e$ are the crosstalk constants.

and $M_{i j}$ is expressed using constants $a, b, \ldots, e$ :

$$
M_{i j}=\frac{1}{1+4 a+4 b+4 c+8 d+4 e} \times \begin{cases}a & (\mathbf{r}=(0,1),(1,0)) \\ b & (\mathbf{r}=(1,1)) \\ c & (\mathbf{r}=(0,2),(2,0)) \\ d & (\mathbf{r}=(1,2),(2,1)) \\ e & (\mathbf{r}=(2,2)) \\ 0 & \text { (other) }\end{cases}
$$

where $\mathbf{r} \equiv\left(n_{x(y)}, n_{z}\right)$ represents the distance from the central channel: $n_{x(y)}$ pixels and $n_{z}$ pixels from the central channel in the $x(y)$ and $z$ directions, respectively.

\section{A.1.2 Hit threshold for tracking}

After the crosstalk correction, only hits with at least two photoelectrons, corresponding to approximately $0.2 \mathrm{MeV}$, are used for tracking. The channel-by-channel variation of the corresponding energy threshold is about $20 \%$ as described in Section 5.1.6.

\section{A.2 Track reconstruction in SciBar}

Track reconstruction in SciBar has two steps: a two-dimensional (2D) track reconstruction and a three-dimensional (3D) track reconstruction.

\section{A.2.1 2D track reconstruction}

The 2D track reconstruction package is called SciBar Cellular Automaton Tracking (sbcat), originally developed for the SciBar detector in the K2K experiment [105]. The cellular automaton algorithm [115] is a discrete model with a dynamical system which evolves in discrete steps. The system consists of a regular grid of cells, and each cell has a finite number of states. These states of the cells are updated according to a common rule. The state of a cell at a given time depends only on its own state and its neighbors at the previous step. 
(A) Initial state

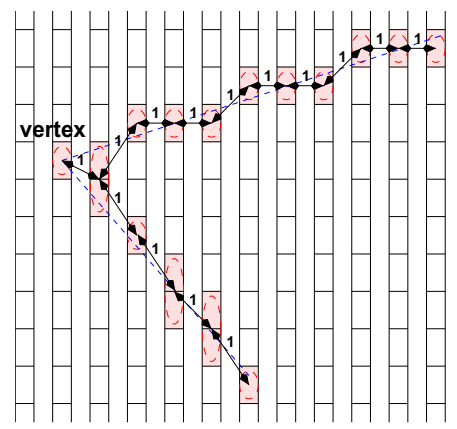

$\square$ hit

cluster

-..- true track

$\therefore$ cell with its state value
(B) After one step

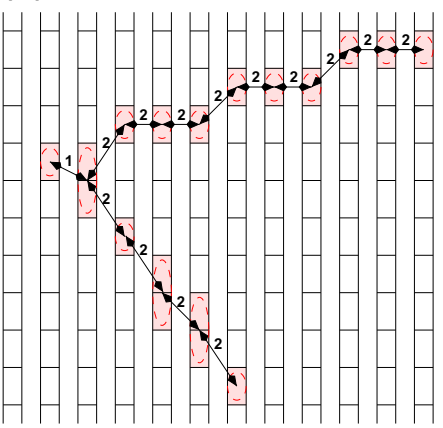

(C) Final state

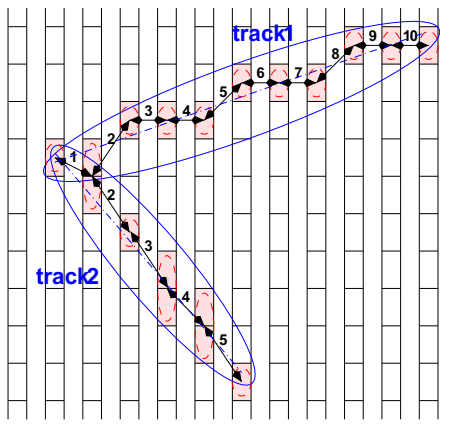

reconstructed segments

reconstructed track

Figure A.2: Illustrations of the cellular automaton tracking for each step. (A) The CAT cells are initialized with a state value of one. (B) The state value of the CAT cells is evolved by the CAT rule. (C) Finally, the track is reconstructed by connecting the CAT neighbors in order of their state values.

Prior to applying the cellular automaton tracking, we make clusters of adjacent hits in each plane since a slanting track may pass through more than one scintillator strip in a plane. We define the cell, neighbor, and evolution rule for the cellular automaton tracking as follows.

- CAT cell: A straight line segment connecting two clusters in adjacent layers. The CAT cell is neither a hit nor a cluster. To take into account detector inefficiency and geometrical acceptance due to the reflective coating, the line segment is allowed to skip over one layer. If the angle of the line segment with respect to the $z$ direction is larger than 1.1 radians, the clusters must contain more than one hit per layer.

- CAT neighbor: Only the CAT cells with a common end are considered as the CAT neighbor. In addition, the $\chi^{2}$ calculated from a linear least square fit to the three clusters belonging to two CAT cells is required to be less than 4.5.

- CAT rule: The CAT cells are initialized with a state value of one. At each time step, the algorithm looks at the upstream region of each CAT cell and looks for the CAT neighbors. If there is the CAT neighbors with the same state value, the state value of the CAT cell is incremented by one unit. The evolution stops when there is no more CAT neighbor with the same state value.

Figure A.2 shows illustrations of the cellular automaton tracking for each step. The CAT cells are indicated as arrows. First, the CAT cells are initialized with a state value of one. The state value of the CAT cells is evolved by the CAT rule at each time step. Finally, the track candidates start from the CAT cell with the lowest state value, and are reconstructed by connecting the CAT neighbors in order of their state values: $1-2-3-\cdots$. The procedure runs along the CAT cell tree until all combinations are considered. 


\section{A.2.2 3D track reconstruction}

$3 \mathrm{D}$ tracks are reconstructed by matching the timing and $z$-edges of the $2 \mathrm{D}$ tracks. The time of each 2D track is calculated as the average time of hits which are associated with the $2 \mathrm{D}$ track. The timing difference between two $2 \mathrm{D}$ tracks is required to be less than $50 \mathrm{nsec}$. The $z$-edge difference between two 2D track must be less than $6.6 \mathrm{~cm}$ for upstream and downstream edges. Reconstructed tracks are required to have at least three-layer penetration, and therefore the minimum length of a reconstructed track is $8 \mathrm{~cm}$ in the beam direction.

If more than one 2D tracks are matched to one 2D track in the other view, the procedure to choose the best combination is as follows. The combination which has smaller $z$-edge difference is favored. If two combinations have same $z$-edge difference, the $\chi^{2}$ of the energy deposition per unit length between two $2 \mathrm{D}$ tracks is calculated. The combination which has smaller $\chi^{2}$ is favored.

\section{Track edge definition}

After pairing 2D tracks, we define the upstream and downstream edges of the 3D track as follows. At first, the upstream (downstream) $z$-edge, $z_{i(f)}$, is defined as the $z$ position of the most upstream (downstream) hit among two $2 \mathrm{D}$ tracks, and therefore they are discrete. Once the $z$-edges of the 3D track are defined, the $x$ and $y$ positions of the upstream (downstream) edge of the $3 \mathrm{D}$ track, $x_{i(f)}$ and $y_{i(f)}$, are calculated by extrapolating the $2 \mathrm{D}$ tracks to the defined $z$-edges:

$$
\begin{aligned}
& x_{i(f)}=z_{i(f)} \cdot \tan \theta_{z x}+x_{0}, \\
& y_{i(f)}=z_{i(f)} \cdot \tan \theta_{z y}+y_{0},
\end{aligned}
$$

where $\tan \theta_{z x}$ and $x_{0}$ are the slope and offset of the $2 \mathrm{D}$ track in the $z$ - $x$ projection, obtained by fitting the $2 \mathrm{D}$ track with a straight line. $\tan \theta_{z y}$ and $y_{0}$ are the ones for the $2 \mathrm{D}$ track in the $z-y$ projection.

\section{Fiber attenuation correction}

Before the 3D track reconstruction, the position of each hit along the fiber is unknown, and thus we cannot correct for the light attenuation effect in the WLS fiber. However, once the 2D track pair is found, the 3D position of each hit is defined; i.e. the position of each hit along the fiber is calculated using the $2 \mathrm{D}$ track in the other view. The charge information after correcting for the fiber attenuation is calculated as

$$
Q^{\prime}=Q \exp \left(\frac{\left|X Y-X Y_{0}\right|}{\lambda}\right)
$$

where $\lambda$ is the attenuation length of the WLS fiber, $X Y$ is the position of the hit along the fiber, $X Y_{0}$ is the reference position which is set to the scintillator edge close to the MA-PMT.

\section{A.2.3 Track edge correction}

As described in Section A.1.1, we correct for the effect of crosstalk before track reconstruction. However, because of the Poisson statistics of photoelectrons, the correction 


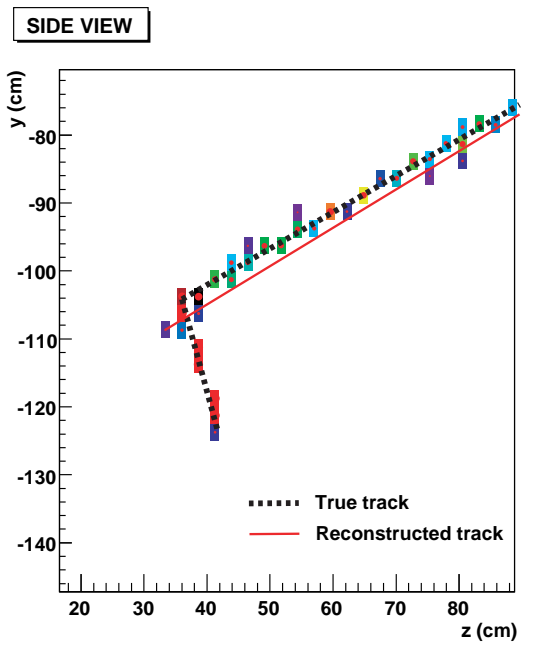

Figure A.3: Event display of a reconstructed track before the track edge correction.

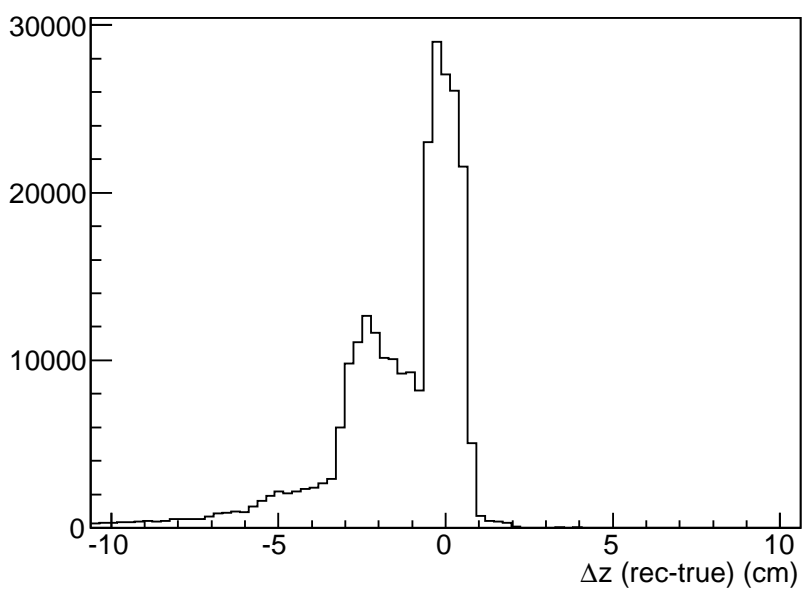

Figure A.4: Upstream z-edge difference between the true and reconstructed muon tracks before the track edge correction.

using the matrix cannot correct for the crosstalk perfectly, and therefore the track edge reconstruction is affected by the residual crosstalk hits. Figure A.3 shows an event display of a reconstructed track. The upstream $z$-edge of the track is shifted toward upstream by one layer due to the residual crosstalk hit. Figure A.4 shows the difference between the true and reconstructed upstream $z$-edge of the muon track estimated with the MC simulation. The second peak around $\Delta z=-2.6 \mathrm{~cm}$ is due to the residual crosstalk hits. Since we take the most upstream hit as the upstream $z$-edge of the track, the reconstructed $z$-edge is always biased to the same direction due to crosstalk.

Therefore, a track edge correction is applied to avoid such a reconstruction bias. The correction is done by scanning the maximum number of photoelectrons in each layer along the track to find the crosstalk hits since the number of photoelectrons of the residual crosstalk hits is expected to be small. Figure A.5 shows the number of photoelectrons in the strip of the upstream end of the track. A peak below 10 photoelectrons is due to noise hits, mainly crosstalk hits. The procedure of the track edge correction is as follows. If the most upstream hit layer satisfies the following condition, the layer is removed from the track edge calculation, and then the next layer is examined;

- The maximum number of photoelectrons in the layer is less then 10 p.e., or

- the ratio of the maximum number of photoelectrons in the layer to that in the next layer is less than 0.1 .

This is repeated until a layer which doesn't satisfy the condition appears, and then the upstream track edge is re-calculated. Figure A.6 and Figure A.7 show the event display and the upstream $z$-edge difference between the true and reconstructed muon track after the track edge correction, respectively. As seen in the event display, the track is correctly reconstructed by removing the most upstream hit from the calculation. The second peak disappears in the Figure A.7. The correction is also applied to the downstream edge. 


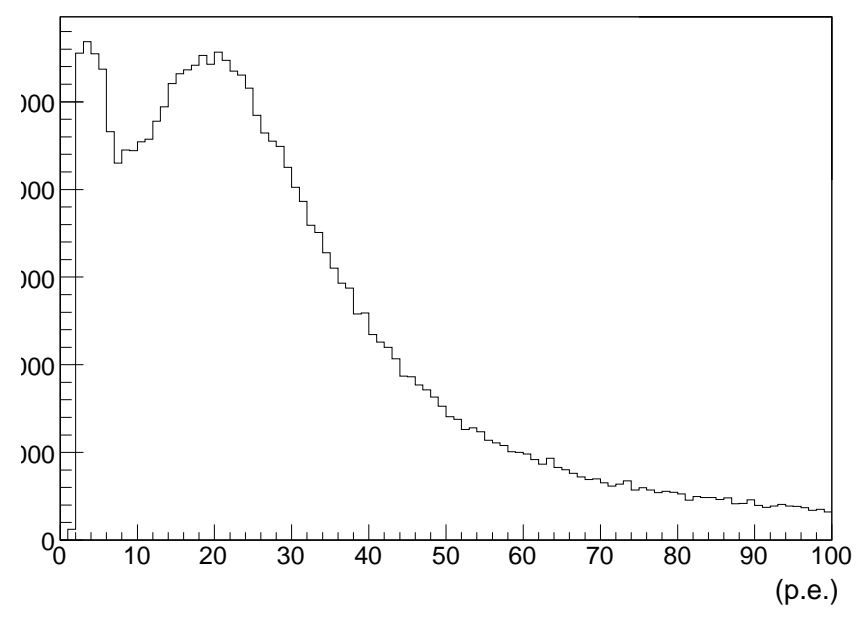

Figure A.5: Number of photoelectrons in the strip of the upstream end of the track.

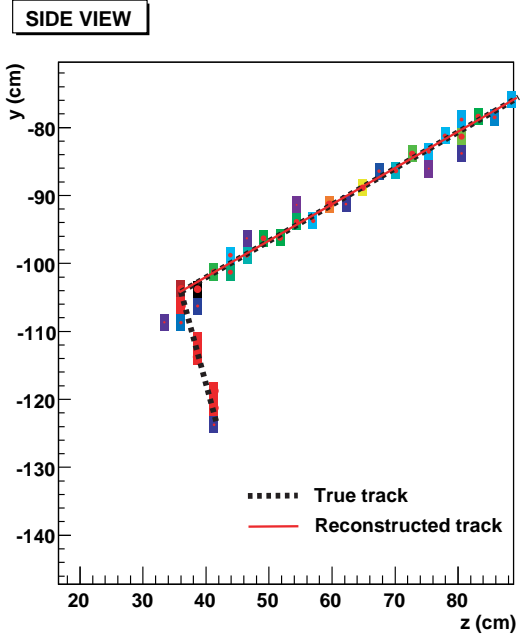

Figure A.6: Event display of a reconstructed track after the track edge correction.

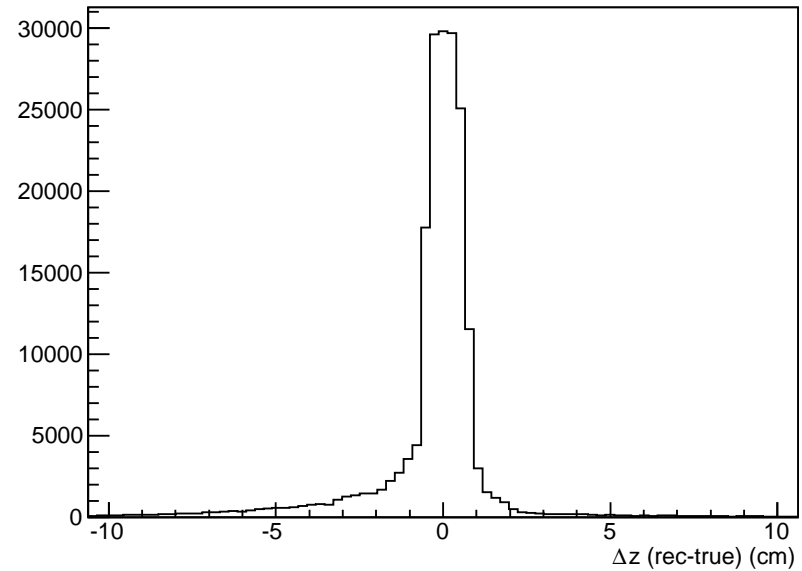

Figure A.7: Upstream z-edge difference between the true and reconstructed muon tracks after the track edge correction.

\section{A.3 Track reconstruction improvement}

In this chapter, we discuss improvements of track reconstruction implemented on top of the default reconstruction package based on the cellular automaton tracking: the kink track and overlapping track reconstructions.

\section{A.3.1 Kink track reconstruction}

Since the cellular automaton tracking reconstructs 2D tracks by looking at only the neighbor channels with a simple rule, it is possible to connect all the hits and reconstruct them as one track even if there is a kink along the path. Figure A.8 shows an example of such an event. In this case, the kink is the neutrino interaction vertex, a muon goes 

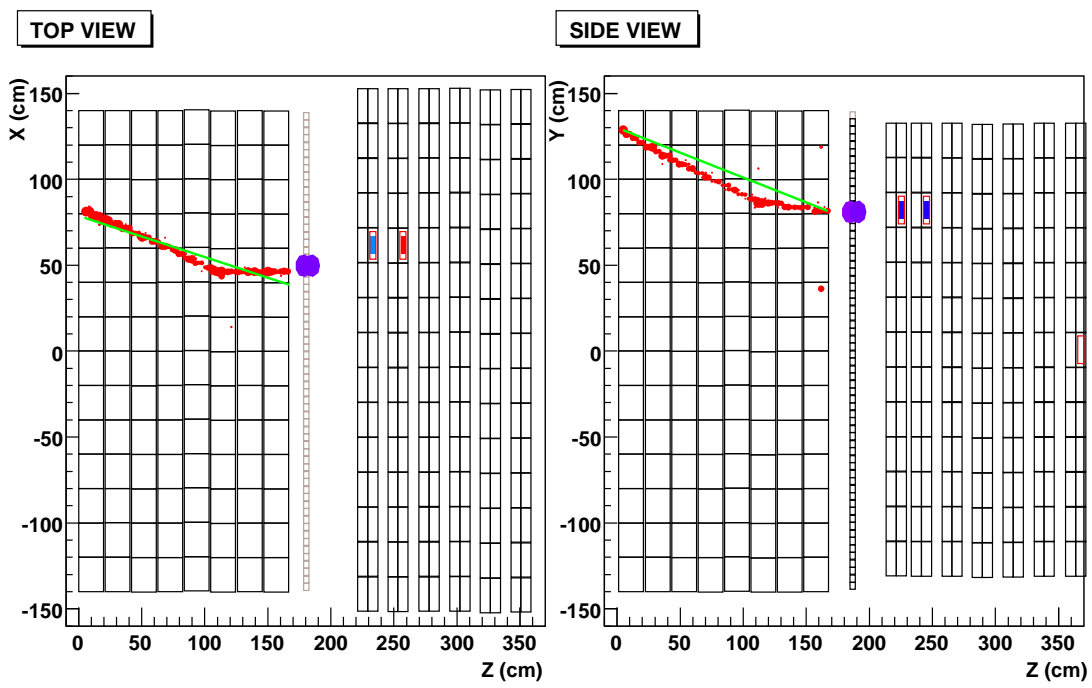

Figure A.8: Example of reconstruction failure due to a kink. The solid (green) line shows the reconstructed $3 \mathrm{D}$ track by the default tracking.

(a) Reduced chi-squared

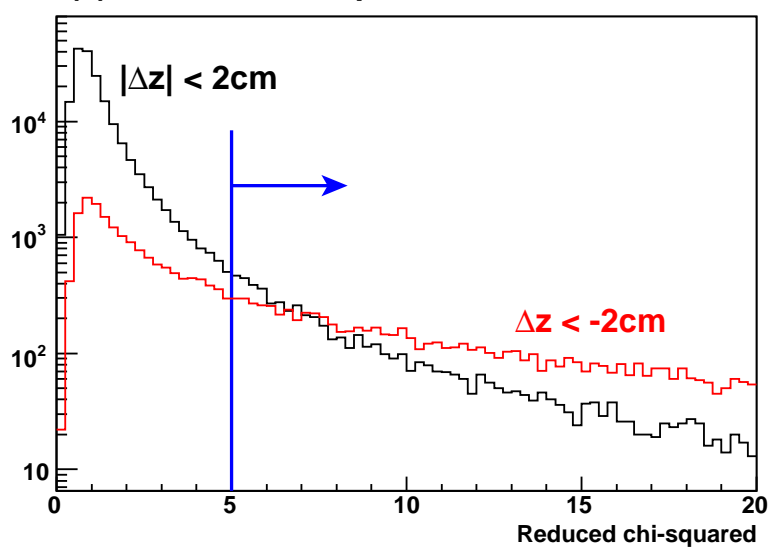

(b) Vertex $z$ resolution (rec.-true)

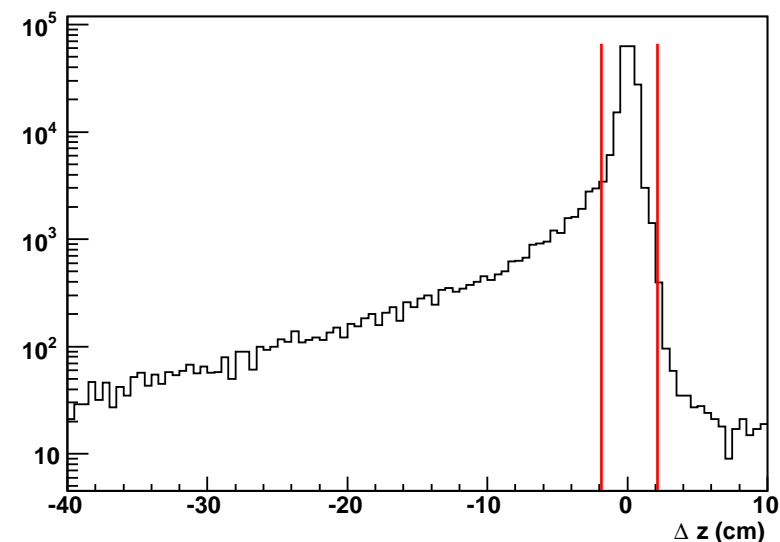

Figure A.9: Reduced $\chi^{2}$ and vertex $z$ resolution of the SciBar-MRD matched track for the MC events.

backward, and a proton is directed forward. However, the event is reconstructed as a one-track event. The interaction vertex is also mis-reconstructed because we define the vertex as the most upstream end of the muon candidate. In order to improve this, a method to find the kink in the already reconstructed track is developed as follows.

First, we compute $\chi^{2}$ of each reconstructed 2D track. Figure A.9 shows the reduced $\chi^{2}$ of the SciBar-MRD matched track for the MC events. Two distributions in Figure A.9 (a) correspond to events with the correctly reconstructed vertex $(|\Delta z|<2 \mathrm{~cm})$ and events with the reconstructed vertex shifted upstream $(\Delta z<-2 \mathrm{~cm})$, respectively. Such a misreconstructed event is mainly due to a backward-going particle which is reconstructed as a part of the SciBar-MRD matched track, and its reduced $\chi^{2}$ tends to be large. If the reduced $\chi^{2}$ is greater than 5 , we apply the kink search algorithm described below.

The next step is to find the kink position along the reconstructed track. Figure A.10 


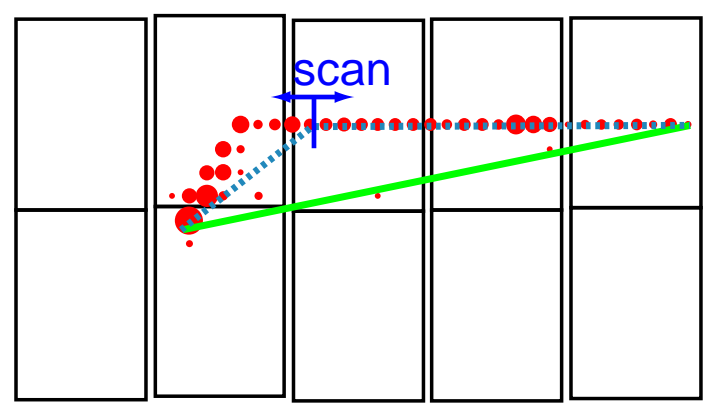

Figure A.10: Illustration of a method to find a kink.

illustrates the method. We postulate a given kink candidate position and draw a kinked line as shown in the figure. The residual sum of squares for as a function of the kink $z$-position $z_{\text {kink }}$ is defined as

$$
s\left(z_{\text {kink }}\right)=s_{\text {top }}+s_{\text {side }}=\sum_{i=1}^{N_{\text {top }}}\left(x_{i}-x_{\text {exp }}\left(z_{\text {kink }}, z_{i}\right)\right)^{2}+\sum_{i=1}^{N_{\text {side }}}\left(y_{i}-y_{\text {exp }}\left(z_{\text {kink }}, z_{i}\right)\right)^{2},
$$

where $x_{i}$ and $y_{i}$ are the average positions of hits in each layer. $x_{\exp }\left(z_{i}\right)$ and $y_{\exp }\left(z_{i}\right)$ are the $x$ and $y$ positions at $z=z_{i}$, expected by the given kinked line. We move the kink candidate along the hits and search the kink position where the residual sum of square is minimized.

Due to multiple scattering in the detector, the track can be bent with a small angle. Figure A.11 shows the opening angle of the kinked line (angle between two segments of the kinked line) for reconstructed tracks which consist of two particles (solid line) and one particle (dashed line). To reduce the possibility of breaking a true track into two, the requirement on the opening angle of the kinked line is imposed; the opening angle must be less than 165 degrees. In addition, since the angle resolution is poor for short tracks, we impose the requirement on the length of two segments of the kinked line; both segments are required to be longer than $15 \mathrm{~cm}$. If both the requirements are satisfied, the track is broken into two tracks at the kink position.

\section{A.3.2 Overlapping track reconstruction}

If two tracks are overlapping each other in one view, the shorter track (second track) is hardly reconstructed as a 3D track even if the second track is clearly seen in the other view as shown in Figure A.12. This is because we require matching of $z$-edges between $2 \mathrm{D}$ tracks in the top and side views.

The method to improve track reconstruction for tracks which are overlapped with another track in one view is as follows. We use the light yield infromation to find the overlapping second track. Figure A.13 shows the profile of the number of photoelectrons per layer along the track in the overlapping view. If two tracks overlap, the light yield is expected to be doubled. Thus, we search for the dropping point of the photoelectron profile where the following conditions are satisfied, by scanning along the track.

$$
\frac{\text { p.e. in }(i-1) \text {-th layer }}{\text { p.e. in } i \text {-th layer }}<2 \quad, \quad \frac{\text { p.e. in }(i-2) \text {-th layer }}{\text { p.e. in } i \text {-th layer }}<2
$$




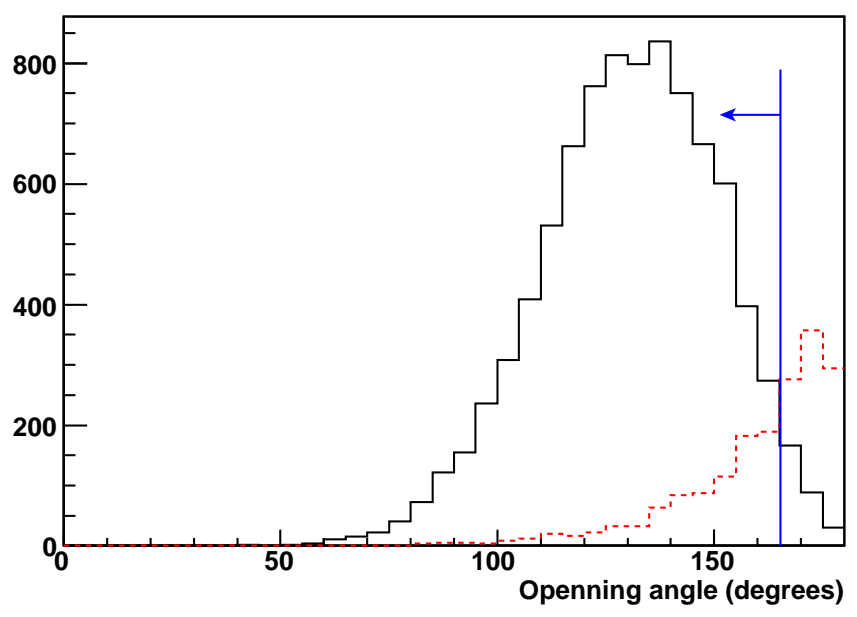

Figure A.11: Opening angle of the kinked line (angle between two segments of the kinked line) for reconstructed tracks which consist of two particles (solid line) and one particle (dashed line).
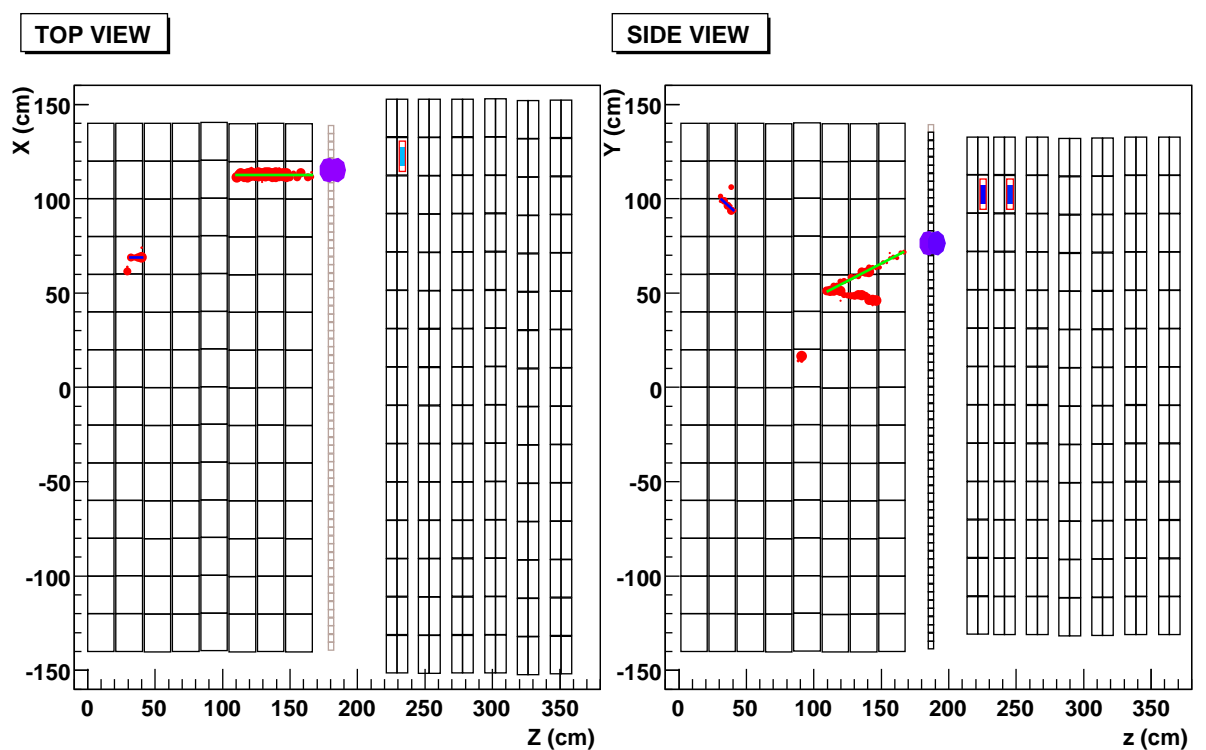

Figure A.12: Example of reconstruction failure due to track overlap. The solid (green) line shows the reconstructed 3D track by the default tracking.

The second requirement is added in order to reduce the fake due to inefficiency. If the $z$-position of the dropping point is matched with that of the track edge in the other view, we consider the point is the track edge.

\section{A.3.3 Reconstruction performance}

Figure A.14 shows the true neutrino energy distribution for $\nu_{\mu} \mathrm{CC}$ events in which at least one track is reconstructed (left), and the $\nu_{\mu} \mathrm{CC}$ efficiency as a function of true 


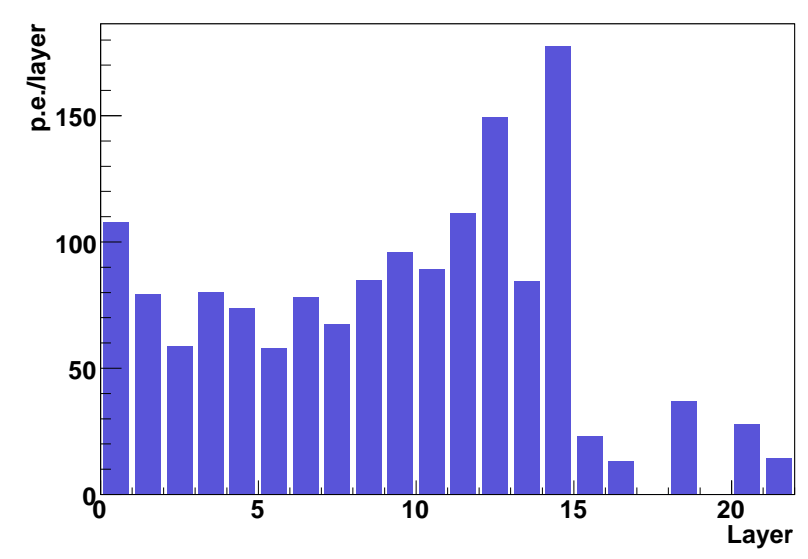

Figure A.13: Opening angle of the kinked line (angle between two segments of the kinked line) for reconstructed tracks which consist of two particles (solid line) and one particle (dashed line).
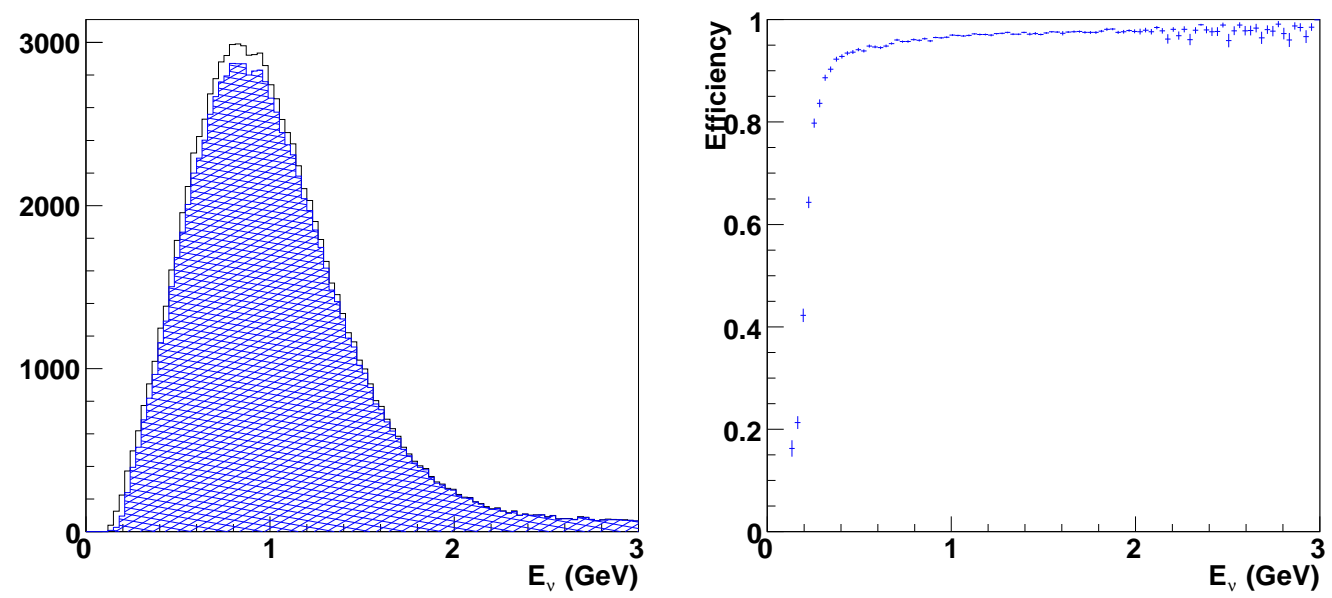

Figure A.14: True neutrino energy distribution for $\nu_{\mu} \mathrm{CC}$ events in which at least one track is reconstructed (left) and $\nu_{\mu} \mathrm{CC}$ efficiency as a function of true neutrino energy (right).

neutrino energy (right), estimated with the MC simulation. The efficiency is defined as

$$
\nu_{\mu} \text { CC efficiency } \equiv \frac{\text { the number of } \nu_{\mu} \text { CC events reconstructed }}{\text { the number of } \nu_{\mu} \text { CC events generated in the fiducial volume }}
$$

where the fiducial volume is defined in Section 7.2.2. According to the MC simulation, $96 \%$ of charged current interactions in SciBar are reconstructed to have at least one track, averaged over entire neutrino energy spectrum. 


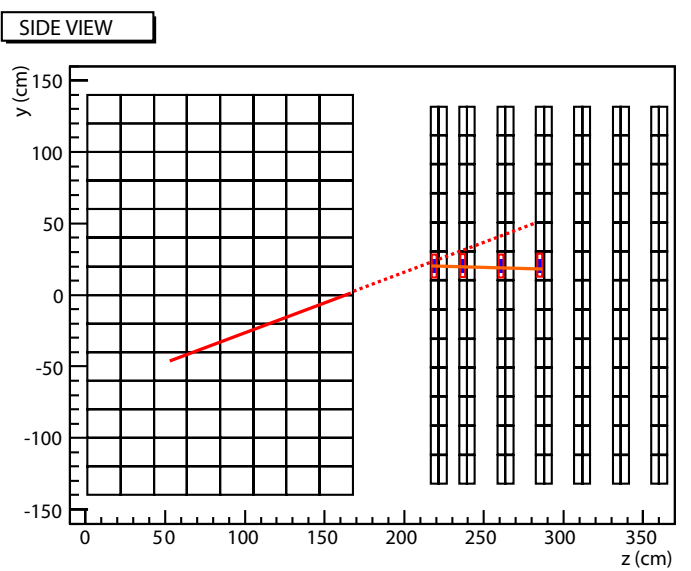

Figure A.15: Schematic drawing of the matching between a SciBar track and an MRD track.

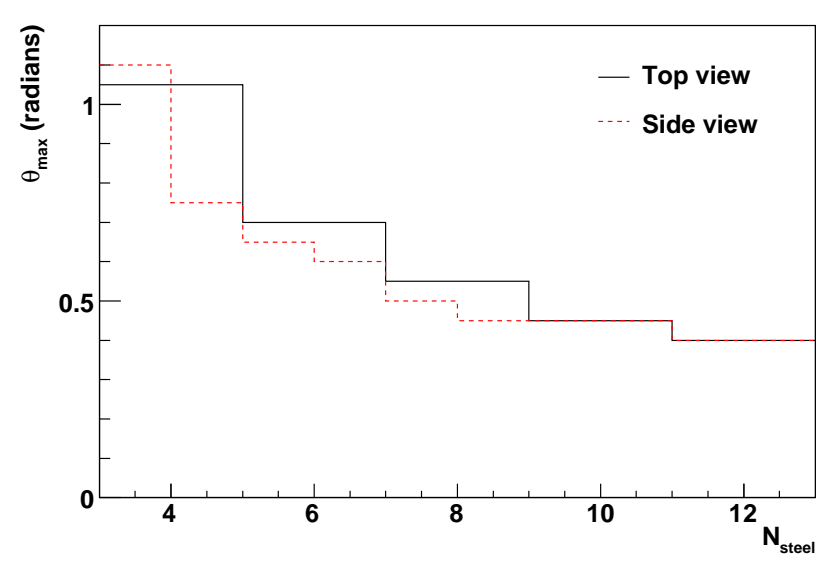

Figure A.16: Maximum angle difference between the SciBar and MRD tracks allowed for matching as a function of the number of steel plates. The solid and dashed lines show the requirements in the top and side view, respectively.

\section{A.4 Matching between SciBar and MRD tracks}

The reconstructed 3D track in SciBar which is matched with a track or hits in the MRD is defined as the SciBar-MRD matched track. The matching procedure is done at the 2D track level. If the downstream edge of the SciBar 2D track is on the last two layers of SciBar, we search for a MRD 2D track or hits which are matched to the SciBar track, using the timing and position information. There are two methods: the track-based matching and the hit-based matching.

\section{A.4.1 Track-based matching}

If MRD tracks are found, we examine the matching between the SciBar and MRD tracks. For matching a MRD track to a SciBar track, the upstream edge of the MRD track is required to be on either one of the first two layers of the MRD. The transverse distance between the SciBar and MRD tracks at the first layer of the MRD must be less than $30 \mathrm{~cm}$. The requirement on the difference between track angles with respect to the beam direction is given by

$$
\left|\theta_{\mathrm{MRD}}-\theta_{\mathrm{SB}}\right|<\theta_{\max }
$$

where $\theta_{\max }$ is a function of the length of the MRD track, varying between 0.4 radian and 1.1 radians as shown in Figure A.16. The function is obtained as the $3 \sigma$ boundary of the distribution in each MRD scintillator plane with the MC simulation. For the timing matching, the timing difference between the SciBar and MRD tracks is required to be within 100 nsec. For track reconstruction in the MRD, at least two hit layers in each view are needed, and thus this matching method is used for tracks which penetrate at least three steel plates. 


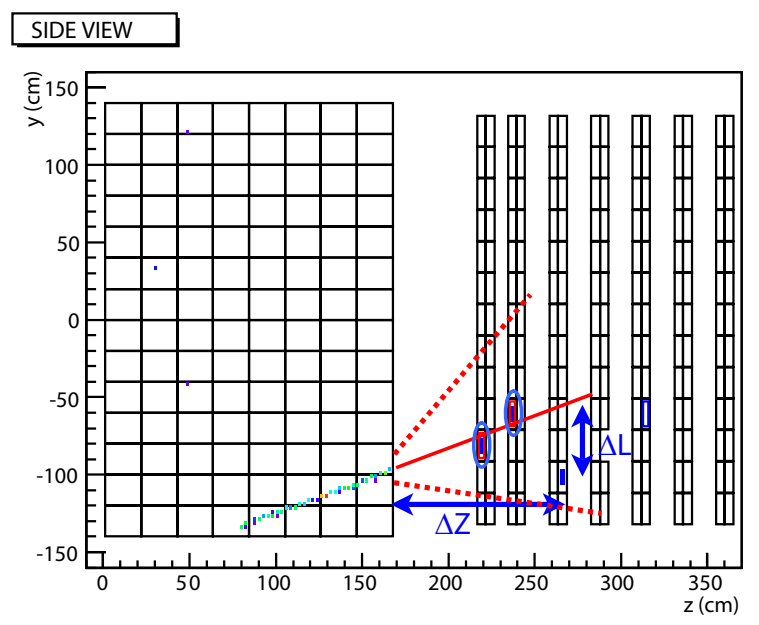

Figure A.17: Schematic drawing of the matching between a SciBar track and hits in the MRD. The solid line shows the extrapolation from the SciBar track, and the dashed lines show the boundaries for the matching.

\section{A.4.2 Hit-based matching}

If no MRD track is found, we extrapolate the SciBar track to the MRD and search for nearby contiguous hits in the MRD identifying a short muon track. The requirement for the hit position is as follows.

$$
\tan \left(\theta-\theta_{\max }\right) \times \Delta Z-Z_{0}<\Delta L<\tan \left(\theta+\theta_{\max }\right) \times \Delta Z+Z_{0}
$$

where $\theta$ is the angle of the SciBar $2 \mathrm{D}$ track with respect to the beam direction. $\Delta Z$ is the distance between the downstream edge of SciBar and the MRD hit position in the $z$ dimension. $\Delta L$ is the distance between the extrapolated position and the MRD hit position in the $x$ or $y$ dimension. $\theta_{\max }$ is set to 0.5 radian, and $Z_{0}$ is set to $10 \mathrm{~cm}$. The requirement corresponds to a cone with an aperture of \pm 0.5 radian and a transverse offset within $10 \mathrm{~cm}$ of the extrapolated SciBar track at the upstream edge of the MRD as shown in Figure A.17. The matching criteria imposes a muon momentum threshold of $350 \mathrm{MeV} / c$. 


\section{Appendix B}

\section{Low $Q^{2}$ Data Excess in the $\mu+p$ Sample}

In this chapter, we investigate the low $Q^{2}$ data excess found in $\mu+p$ sample.

Figure B.1 shows length and angle of the second track in $Q^{2}<0.15 \mathrm{GeV} / c^{2}$ in the $\mu+p$ sample. In the 2 nd track angle distribution, the data excess appears in between 30 degrees and 90 degrees, at relatively large angle. To enrich the data excess, events in which the second track angle is between 30 degrees and 90 degrees are selected.
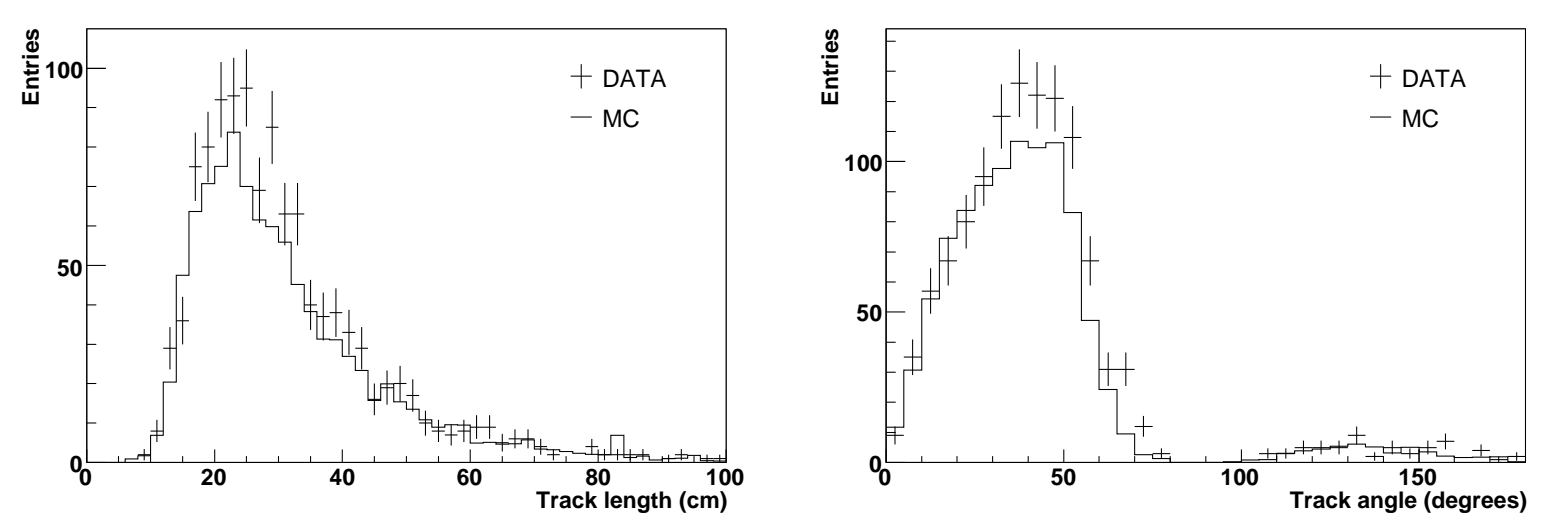

Figure B.1: Length (left) and angle (right) of the second track in $Q^{2}<0.15 \mathrm{GeV} / c^{2}$ in the $\mu+p$ sample.

Figure B.2 shows the vertex distributions for events the data-excess enriched sample. No localization of the data excess is found. Figure B.3 shows $d E / d x$ of the first and second tracks for the data-excess enriched sample. The data excess is at $\sim 2 \mathrm{MeV} / \mathrm{cm}$ for the first track and at $\sim 6 \mathrm{MeV} / \mathrm{cm}$ for the second track, and thus the event really looks like $\mu+p$. Figure B.4 shows vertex activity, the maximum deposited energy in a strip around the vertex, for the data-excess enriched sample. The data excess is seen at larger vertex activity (above $20 \mathrm{MeV}$ ), and the activity is actually located at the vertex. This indicates the existence of an additional low energy proton from the vertex. 

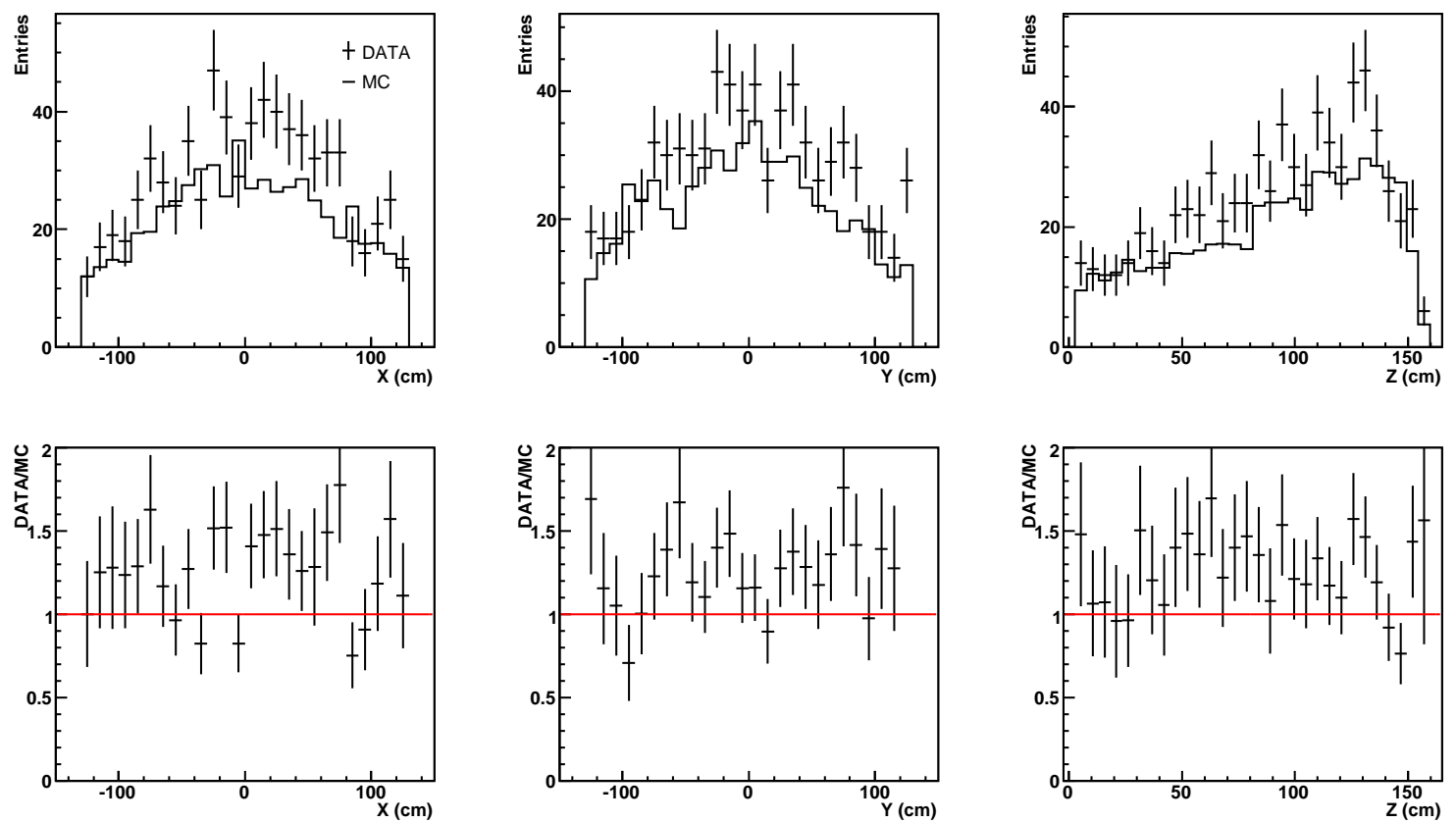

Figure B.2: Vertex distributions for the data-excess enriched sample.
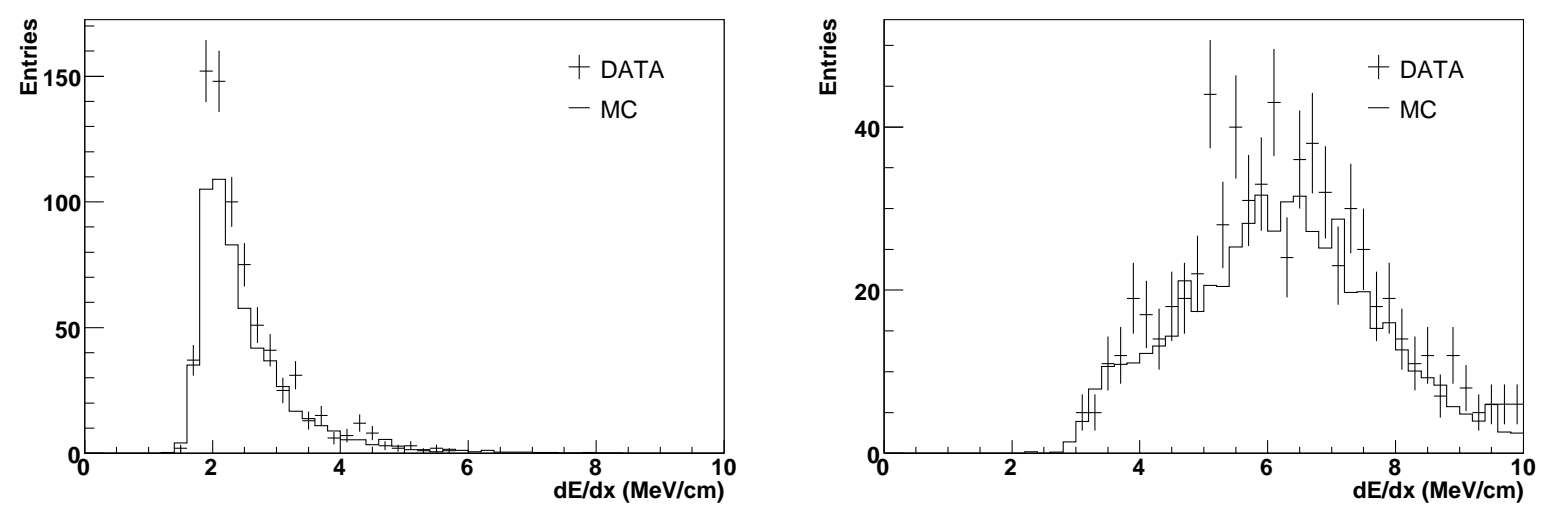

Figure B.3: $d E / d x$ of the first (left) and second (right) tracks for the data-excess enriched sample. 


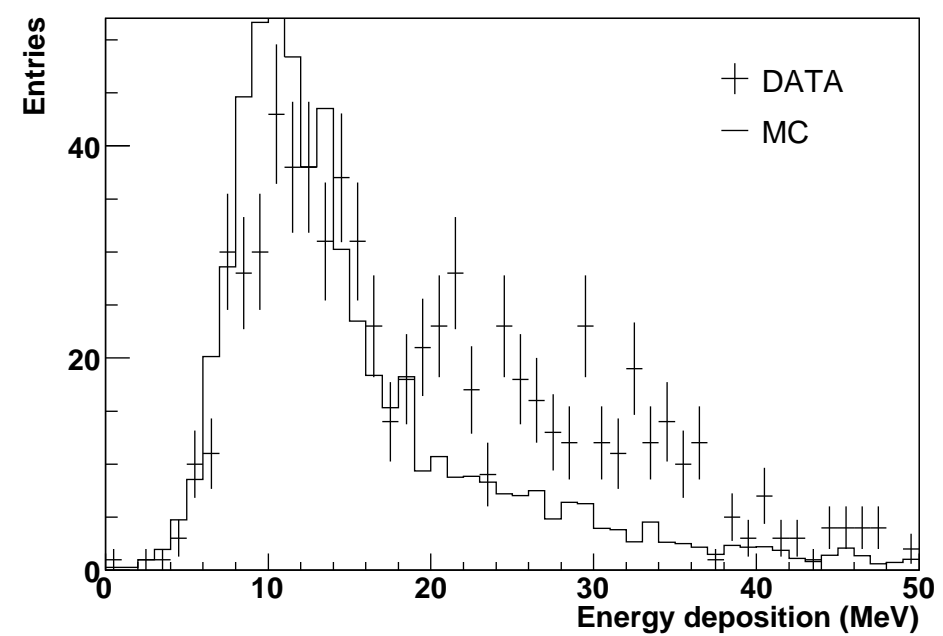

Figure B.4: Vertex activity for the data-excess enriched sample.

In summary, the features of the excess events are as follows.

- Two tracks with an additional large energy deposition at the vertex.

- The first track is MIP-like and has small angle.

- The second track is proton-like and has lage angle (greater than 30 degrees).

- The vertex activity indicates the existence of an additional low energy proton.

A possible candidate of the excess event is $\mathrm{CC}$ resonant pion production where the pion is absorbed in the nucleus. In such an event, two or more additional nucleons should be emitted after the pion is absorbed, which is currently not simulated in NEUT. These protons produced by pion absorption might be the origin of the large angle second track or the large vertex activity. On the other hand, in the $\mathrm{MC}$ simulation, the corresponding events might be reconstructed as one track events without nucleons produced by pion absorption. However, there are approximately 10,000 events at small $Q^{2}$ in the one track sample, while this event migration is at a level of a few hundred events. Therefore, it is difficult to find these events in the one track sample. 


\section{Appendix C}

\section{Kinematic Distributions after the MC Tuning}

In this chapter, we show several kinematic distributions for each sub-sample after the MC tuning described in Section 7.4.

Figure C.1 shows muon momentum and angle with respect to the neutrino beam for the one track sample. Figure C.2 shows muon momentum, angle with respect to the neutrino beam, the second track length, and the second track angle with respect to the neutrino beam for the $\mu+p$ sample. Figure C.3 and Figure C.4 show the kinematic distributions for the $\mu+\pi$ events with and without vertex activity, respectively.
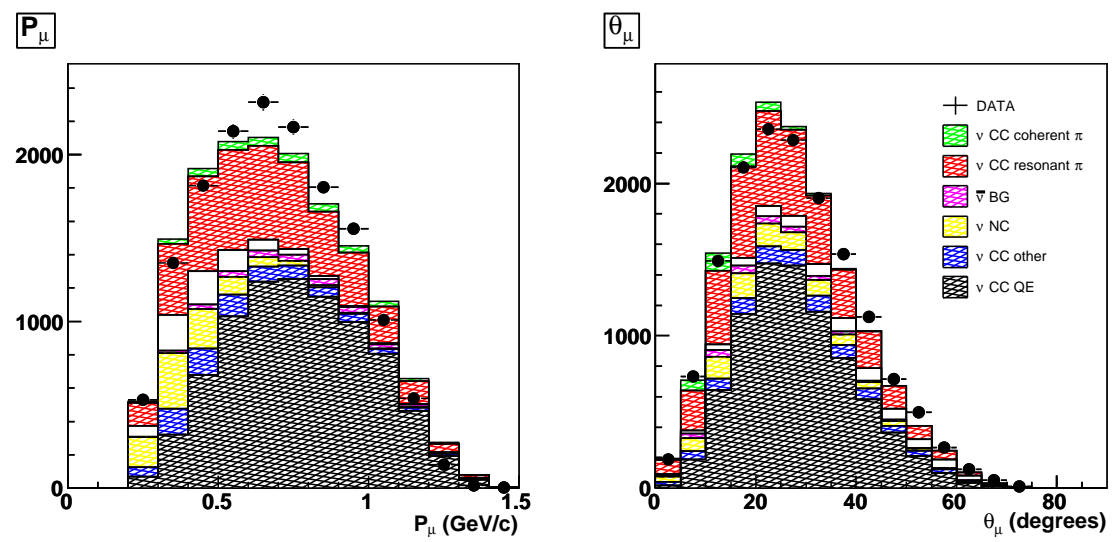

Figure C.1: Muon momentum and angle distributions for the one track events. 


\section{$\mathbf{P}_{\mu}$}

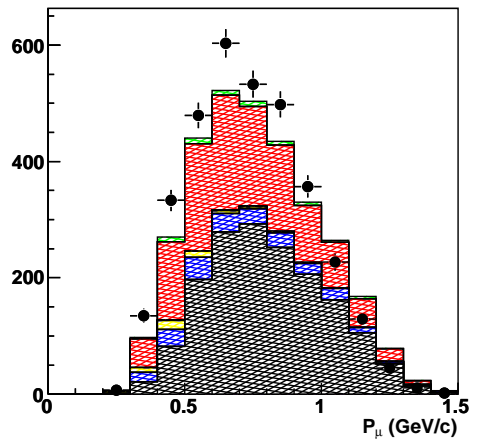

$L_{\text {2nd }}$

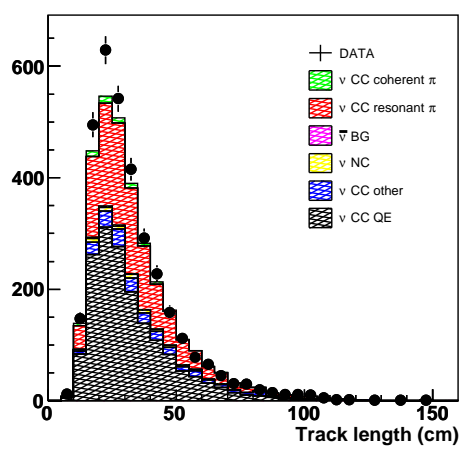

$\theta_{\mu}$

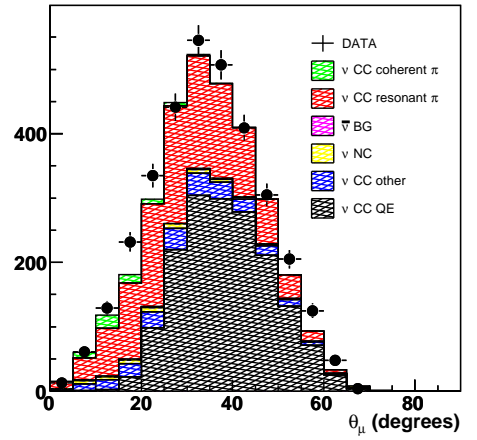

$\theta_{\text {2nd }}$

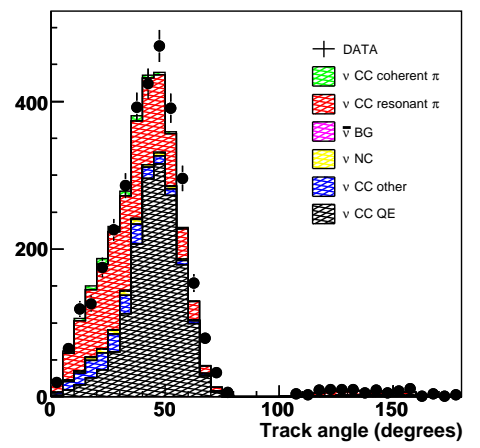

Figure C.2: Muon momentum and angle, 2nd track length, and 2nd track angle distributions for the $\mu+p$ events.

$\mathbf{P}_{\mu}$

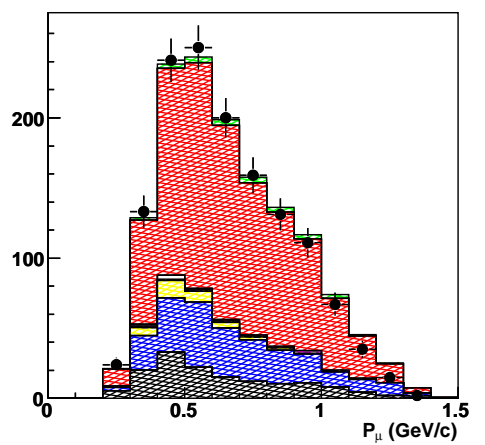

$L_{\text {2nd }}$

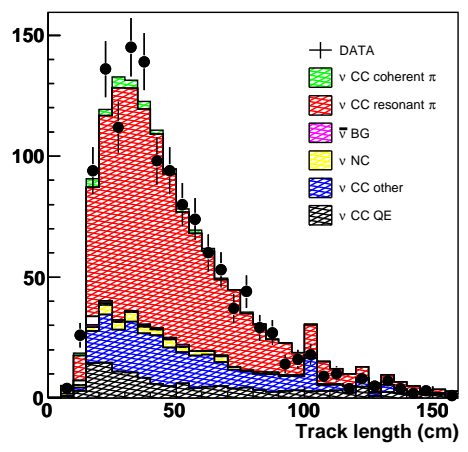

$\theta_{\mu}$

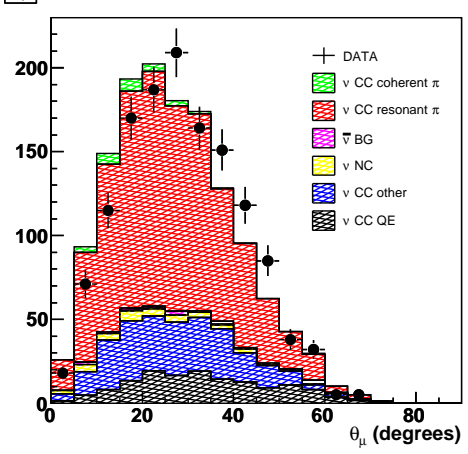

$\theta_{2 \text { nd }}$

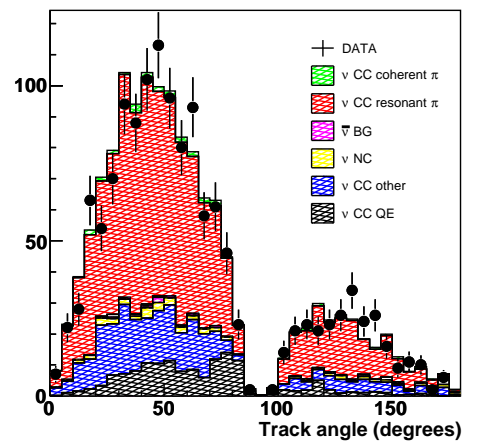

Figure C.3: Muon momentum and angle, 2nd track length, and 2nd track angle distributions for the $\mu+\pi$ events with vertex activity. 

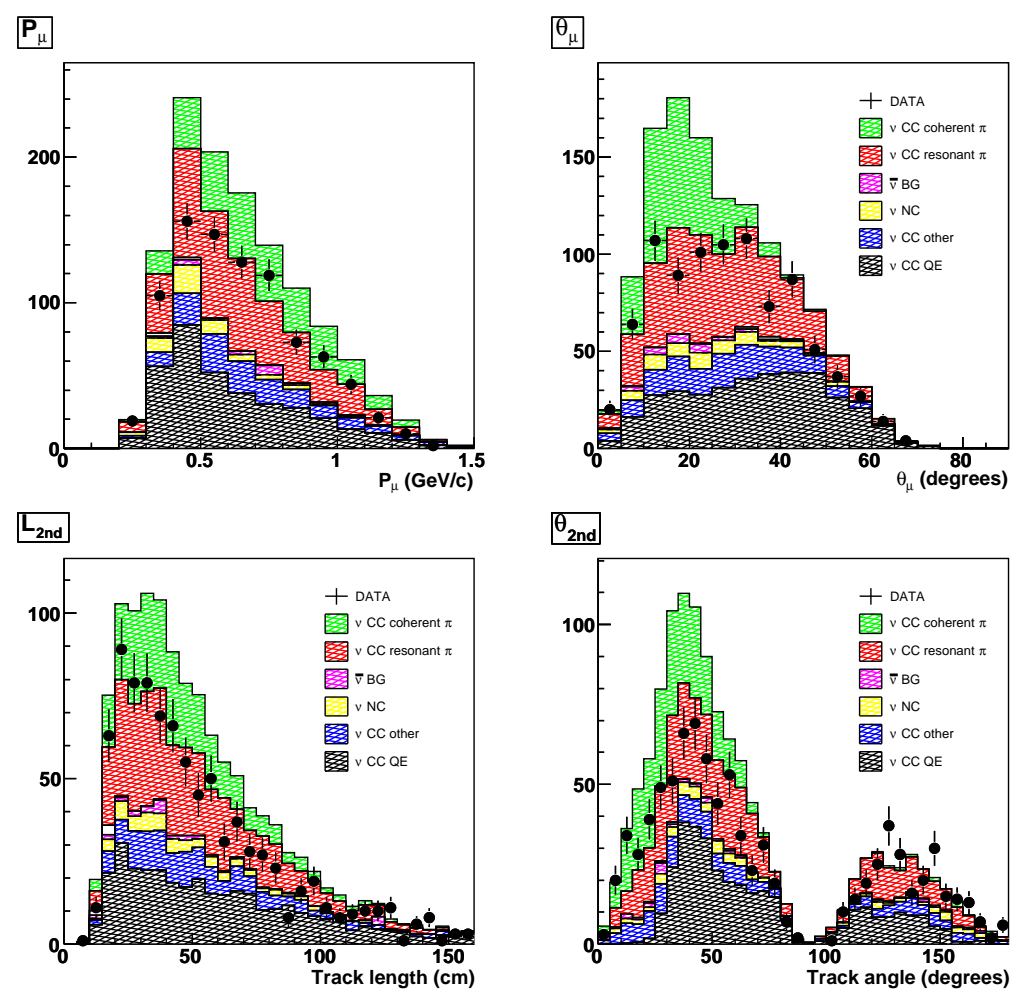

Figure C.4: Muon momentum and angle, 2nd track length, and 2nd track angle distributions for the $\mu+\pi$ events without vertex activity. 


\section{List of Tables}

1.1 Past measurements of charged current resonant pion production by neutrinos. 9

1.2 Measured cross section ratios of charged current resonant charged pion production to charged current quasi-elastic scattering in the K2K experiment. 10

1.3 List of past measurements of coherent pion production. . . . . . . . . . . . 11

2.1 History of the SciBooNE experiment. . . . . . . . . . . . . 16

3.1 Sanford-Wang parameters used in the simulation . . . . . . . . . . . . . . 21

3.2 Feynman scaling parameters used in the simulation . . . . . . . . . . . . 21

3.3 Neutrino-producing decay modes considered in the simulation. . . . . . . . 22

4.1 Expected number of $\nu_{\mu}$ interactions in the SciBar fiducial volume at Sci-

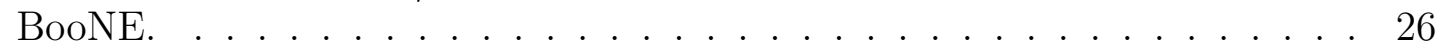

5.1 Specifications of the SciBar detector . . . . . . . . . . . . 35

5.2 Specifications of the MRD detector. . . . . . . . . . . . . . . . . . 44

6.1 Beam quality cuts and fractions of the total number of protons on target that fail each cut. . . . . . . . . . . . . . . . 50 50

6.2 Summary of SciBooNE data-taking. The table shows the number of protons on target (POT) collected after application of data quality cuts, as described in the text. . . . . . . . . . . . . . . . . 53

7.1 Best fit values and errors of the fitting parameters . . . . . . . . . . . 77

7.2 Event selection summary for the MRD stopped charged current coherent pion sample. . . . . . . . . . . . . . . . . . . 82

7.3 Event selection summary of MRD penetrated charged current coherent pion sample. . . . . . . . . . . . . . . . . . . 84

7.4 Summary of the systematic errors in the charged current coherent pion cross section ratio. . . . . . . . . . . . . . . . . . 87

7.5 Systematic errors in the charged current coherent pion cross section ratio due to the uncertainties in the detector responses. . . . . . . . . . . . 88

7.6 Systematic errors in the charged current coherent pion cross section ratio due to the uncertainties in the nuclear effects. . . . . . . . . . . . . . 89

7.7 Systematic errors in the charged current coherent pion cross section ratio due to the uncertainties in the neutrino interaction models. . . . . . . . . . 90

8.1 Summary of event samples used for the resonant pion cross section measurement. . . . . . . . . . . . . . . . . . 104 


\section{List of Figures}

1.1 Allowed or excluded regions in the $\tan ^{2} \theta-\Delta m^{2}$ plane from various experiments. . . . . . . . . . . . . . . . . . . 3

1.2 Charged current total cross section divided by $E_{\nu}$ for neutrino nucleon charged current interactions. . . . . . . . . . . . . . 5

1.3 Expected distribution of the reconstructed neutrino energy assuming the CC-QE kinematics, and the energy resolution in the case of no oscillation.

1.4 The diagrams of the signal and background processes for the $\nu_{\mu}$ disappearance study. . . . . . . . . . . . . . . . . . . .

1.5 Expected distribution of the reconstructed neutrino energy in the case of $\Delta m_{23}^{2} \sim 2.7 \times 10^{-3} \mathrm{eV}^{2}$ and $\sin ^{2} 2 \theta_{23} \sim 1 \ldots \ldots \ldots$

1.6 Ratio of the reconstructed neutrino energy distribution with oscillation to one without oscillation. . . . . . . . . . . . . . . 7

1.7 Size of the measurement error in the oscillation parameters, $\sin ^{2} 2 \theta_{23}$ and $\Delta m_{23}^{2}$, as a function of true value of $\Delta m_{23}^{2}$ in various cases of the systematic uncertainty. . . . . . . . . . . . . . . . .

1.8 Experimental results on charged current resonant pion production cross sections in the neutrino energy region of a few $\mathrm{GeV}$. . . . . . . . . . . 9

1.9 Cross section for $\nu_{\mu}{ }^{12} \mathrm{C} \rightarrow \mu^{-} \pi^{+12} \mathrm{C}$ interaction. . . . . . . . . . . . . 12

1.10 Existing experimental results on the coherent pion production cross section $\left(E_{\nu}<20 \mathrm{GeV}\right) \ldots \ldots \ldots \ldots \ldots \ldots \ldots$

2.1 Schematic drawing of the experimental setup of SciBooNE. . . . . . . . . 16

2.2 Comparison of the muon neutrino energy spectra at K2K, T2K, and SciBooNE. . . . . . . . . . . . . . . . . . 17

3.1 Neutrino flux predictions at the SciBooNE detector as a function of neutrino energy $E_{\nu}$, normalized per unit area, proton on target (POT) and neutrino energy bin width in neutrino mode (left) and antineutrino mode running. . . . . . . . . . . . . . . . . . .

3.2 Systematic uncertainty in the neutrino energy distribution for the total interactions by muon neutrinos. . . . . . . . . . . . . . . . . 24

4.1 Neutrino-nucleus cross sections per nucleon divided by neutrino energy. . . 26

5.1 Schematic drawing of the SciBooNE detector. . . . . . . . . . . 33

5.2 Schematic drawing of SciBar. . . . . . . . . . . . . . . . . 34

5.3 Schematic drawing of the SciBar readout system. . . . . . . . . . . . 36

5.4 Emission spectrum for the SciBar scintillator. . . . . . . . . . . . . 37 
5.5 Schematic drawing of a scintillator strip. . . . . . . . . . . . . . . 37

5.6 Ratio of the observed energy deposition per unit length $(d E / d x)$ to the expected $d E / d x$ as a function of the expected $d E / d x \ldots \ldots$. . . . . . . 37

5.7 Absorption and emission spectra for Kuraray Y11(200)MS type WLS fiber. 38

5.8 Picture of a front-end board. . . . . . . . . . . . . . . . . . 39

5.9 Picture of a DAQ board. . . . . . . . . . . . . . . . . . . . 39

5.10 Schematic drawing of the SciBar gain monitoring system. . . . . . . . . . . 40

5.11 Measured stability of the LED intensity during the whole data-taking period. 41

5.12 Measured gain stability during the whole data-taking period for a typical MA-PMT channel. . . . . . . . . . . . . . . . . . . . . . 41

5.13 Number of photoelectrons for cosmic-ray muons for a typical channel. . . . 42

5.14 Energy calibration constants for all channels. . . . . . . . . . . . . . 42

5.15 Schematic drawing of the EC module. . . . . . . . . . . . . . . . 43

5.16 Hit finding efficiency as a function of position for a typical scintillator plane. 45

5.17 Event display of a typical muon neutrino charged current quasi-elastic scattering $\left(\nu_{\mu} n \rightarrow \mu^{-} p\right)$ candidate in SciBooNE data. . . . . . . . . . . 46

5.18 Displacement of each SciBar scintillator layer with respect to the most upstream layer. . . . . . . . . . . . . . . . . . . . . . 4 47

$5.19 \pi^{+}$-carbon interaction cross sections as a function of pion kinetic energy. $\quad 49$

6.1 Distributions of the beam quality variables for a typical beam condition. . 52

6.2 History of the accumulated number of protons on target (POT). . . . . . . 53

6.3 Stability of the number of charged current candidate events in SciBar normalized to the number of protons on target. . . . . . . . . . . . . . . 55

6.4 Stability of the number of charged current candidate events in the MRD normalized to the number of protons on target. . . . . . . . . . . 55

7.1 Difference between the reconstructed and true vertex in each projection estimated with the MC simulation. . . . . . . . . . . . . . 58

7.2 Reconstructed vertex distribution of beam-timing events after 1st layer veto

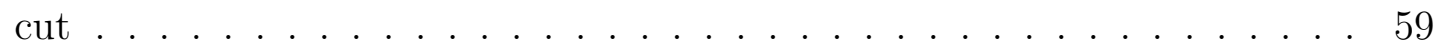

7.3 Event timing distribution of the SciBar-MRD matched events . . . . . . . 59

7.4 Reconstructed vertex distribution of the SciBar-MRD matched sample. . . 60

7.5 $\nu_{\mu}$ CC efficiencies versus true muon momentum and angle for the SciBarMRD matched sample. . . . . . . . . . . . . . . . . . . 61

$7.6 \nu_{\mu} \mathrm{CC}$ efficiencies versus true neutrino energy and the square of fourmomentum transfer for the SciBar-MRD matched sample. . . . . . . . . . 61

7.7 Number of steel planes penetrated by the muon track for the SciBar-MRD matched sample . . . . . . . . . . . . . . . . . . . 62

7.8 Schematic drawings of the MRD stopped, MRD penetrated, and MRD side-escaped events. . . . . . . . . . . . . . . . . . . . 62

$7.9 \nu_{\mu}$ CC efficiencies versus true muon momentum and angle for the MRD stopped and MRD penetrated samples. . . . . . . . . . . . . . 64

$7.10 \nu_{\mu} \mathrm{CC}$ efficiencies versus true neutrino energy and the square of fourmomentum transfer for the MRD stopped and MRD penetrated samples. . 64

7.11 Difference between the reconstructed and true muon momenta for the MRD stopped sample estimated with the MC simulation. . . . . . . . . . . . 65 
7.12 Difference between the reconstructed and true muon angles for the MRD stopped sample estimated with the MC simulation. . . . . . . . . . . .

7.13 Difference between the reconstructed and true $E_{\nu}$ for the MRD stopped sample estimated with the MC simulation. . . . . . . . . . . . . . 65

7.14 Difference between the reconstructed and true $Q^{2}$ for the MRD stopped sample estimated with the MC simulation. . . . . . . . . . . . .

7.15 Difference between the reconstructed and true muon momenta for the MRD penetrated sample estimated with the MC simulation. . . . . . . . . . 66

7.16 Difference between the reconstructed and true muon angles for the MRD penetrated sample estimated with the MC simulation. . . . . . . . . . 66

7.17 Difference between the reconstructed and true $E_{\nu}$ for the MRD penetrated sample estimated with the MC simulation. . . . . . . . . . . . 66

7.18 Difference between the reconstructed and true $Q^{2}$ for the MRD penetrated sample estimated with the MC simulation. . . . . . . . . . . . 66

7.19 Flow chart of event classification. . . . . . . . . . . . . . . . 67

7.20 Track edge distance distribution for all reconstructed tracks in the MRD stopped sample. . . . . . . . . . . . . . . . . . . 68

7.21 Number of tracks from the vertex for the MRD stopped sample. . . . . . . 68

7.22 Expected $d E / d x$ distribution of a cosmic-ray muon and confidence level as a function of $d E / d x$ observed in a scintillator plane. . . . . . . . . . . . . 69

$7.23 \mathrm{dE} / \mathrm{d} x$ of the muon track and proton track. . . . . . . . . . . . . . 70

$7.24 \mathrm{MuCL}$ of the muon track and proton track. . . . . . . . . . . . . . 70

7.25 MuCL of the second track for two-track events in the MRD stopped sample. 71

7.26 Maximum deposited energy in a strip around the vertex for the $\mu+\pi$ events in the MRD stopped sample. . . . . . . . . . . . . . . . . .

7.27 Reconstructed muon momentum and angle before fitting for the one track events, $\mu+p$ events, $\mu+\pi$ events with activity, and $\mu+\pi$ events without activity.

7.28 Reconstructed $Q^{2}$ before fitting for the one track events, $\mu+p$ events, $\mu+\pi$ events with activity and without activity. . . . . . . . . .

7.29 Reconstructed $Q^{2}$ after fitting for the one track events, $\mu+p$ events, $\mu+\pi$ events with activity, and $\mu+\pi$ events without activity. . . . . . .

7.30 Reconstructed muon momentum and angle after fitting for the one track events, $\mu+p$ events, $\mu+\pi$ events with activity, and $\mu+\pi$ events without activity. . . . . . . . . . . . . . . . . 79

$7.31 \Delta \theta_{p}$ for the $\mu+\pi$ events in the MRD stopped sample after fitting. . . . . . 80

7.32 Track angle of the pion candidate with respect to the beam direction for the $\mu+\pi$ events after the charged current quasi-elastic rejection after fitting. 80

7.33 Reconstructed $Q^{2}$ for the $\mu+\pi$ events in the MRD stopped sample after the pion track direction cut and after fitting. . . . . . . . . . . . . 81

7.34 Resolution of reconstructed $Q^{2}$ for the $\mu+\pi$ events in the MRD stopped sample after the pion track direction cut. . . . . . . . . . . . . .

7.35 Reconstructed $Q^{2}$ for the $\mu+\pi$ events in the MRD penetrated sample after the pion track direction cut after fitting. . . . . . . . . . . . .

7.36 Resolution of reconstructed $Q^{2}$ for the $\mu+\pi$ events in the MRD penetrated sample after the pion track direction cut. . . . . . . . . . . . . 
7.37 Neutrino energy spectra and selection efficiencies as a function of neutrino energy for charged current coherent pion events. . . . . . . . . . . .

7.38 Neutrino energy spectra and selection efficiencies as a function of neutrino energy for all charged current events. . . . . . . . . . . . . . . . . . 85

7.39 Reconstructed $Q^{2}$ for $\mu+\pi$ events with vertex activity after CC-QE rejection and pion track direction cut. . . . . . . . . . . . . . . . . . 91

7.40 Reconstructed $Q^{2}$ assuming $\Delta(1232)$, expected $Q^{2}$ resolution, and DATA/MC ratio as a function of reconstructed $Q^{2}$ for $\mu+\pi$ events with vertex activity after CC-QE rejection and pion track direction cut. . . . . . . . . . . . 92

7.41 The variation of the charged current coherent pion cross section ratio for the MRD stopped sample using 1,000 neutrino flux predictions within the systematic uncertainty. . . . . . . . . . . . . . . . . . . 93

7.42 Comparison of our results with theoretical predictions. . . . . . . . . . . 94

8.1 Muon momentum, angle, pion track length, and pion angle distributions for the MRD stopped coherent pion sample. . . . . . . . . . . . . . 97

8.2 Muon angle, pion track length, and pion angle distributions for two different muon momentum regions in the MRD stopped coherent pion sample. . . . 98

8.3 Definition of the kinematic variable $\Delta \phi \ldots \ldots \ldots$

8.4 $\Delta \phi$ distributions for two different muon momentum regions in the MRD stopped coherent pion sample. . . . . . . . . . . . . . . . . . 99

8.5 Distributions of muon momentum and angle for charged current coherent pion production based on the Rein-Sehgal model, and the selection efficiencies for the MRD stopped and penetrated coherent pion samples estimated with the MC simulation.

8.6 Distributions of pion momentum and angle for charged current coherent pion production based on the Rein-Sehgal model, and the selection efficiencies for the MRD stopped and penetrated coherent pion samples estimated with the MC simulation.

8.7 Reconstructed $Q^{2}$ for the one track events, $\mu+p$ events, $\mu+\pi$ events with activity and without activity. . . . . . . . . . . . . . . 102

8.8 Reconstructed $Q^{2}$ for the $\mu+\pi$ events without vertex activity in the MRD stopped sample after the $\Delta \theta_{p}$ and pion track direction cuts. . . . . . . . 102

8.9 Muon momentum and angle for the $\mu+\pi$ events with vertex activity in the MRD stopped sample after the $\Delta \theta_{p}$ cut. . . . . . . . . . . . . . . . 104

8.10 Muon momentum and angle for the $\mu+\pi$ events without vertex activity in the MRD stopped sample after the $\Delta \theta_{p}$ cut. . . . . . . . . . . . . . . . . . 104

8.11 Muon momentum and angle for the one track events in the MRD stopped sample. . . . . . . . . . . . . . . . . . . . 105

8.12 Muon momentum and angle for the $\mu+p$ events in the MRD stopped sample.105

A.1 Illustration of the crosstalk effect. . . . . . . . . . . . . . . . . . . 109

A.2 Illustrations of the cellular automaton tracking for each step. . . . . . . . 110

A.3 Event display of a reconstructed track before the track edge correction. . . 112

A.4 Upstream $z$-edge difference between the true and reconstructed muon tracks before the track edge correction. . . . . . . . . . . . . . . . . . . . . 112

A.5 Number of photoelectrons in the strip of the upstream end of the track. . . 113 
A.6 Event display of a reconstructed track after the track edge correction. . . . 113

A.7 Upstream $z$-edge difference between the true and reconstructed muon tracks after the track edge correction. . . . . . . . . . . . . . . . . . 113

A.8 Example of reconstruction failure due to a kink. . . . . . . . . . . . . . . 114

A.9 Reduced $\chi^{2}$ and vertex $z$ resolution of the SciBar-MRD matched track for the $\mathrm{MC}$ events. . . . . . . . . . . . . . . . . . . . . 114

A.10 Illustration of a method to find a kink. . . . . . . . . . . . . . . 115

A.11 Opening angle of the kinked line for reconstructed tracks which consist of two particles and one particle. . . . . . . . . . . . . . . 116

A.12 Example of reconstruction failure due to track overlap. . . . . . . . . . 116

A.13 Opening angle of the kinked line for reconstructed tracks which consist of two particles and one particle. . . . . . . . . . . . . . . 117

A.14 True neutrino energy distribution for $\nu_{\mu}$ CC events in which at least one track is reconstructed and $\nu_{\mu} \mathrm{CC}$ efficiency as a function of true neutrino energy. . . . . . . . . . . . . . . . . . . . . 117

A.15 Schematic drawing of the matching between a SciBar track and an MRD track. . . . . . . . . . . . . . . . . . . . . 118

A.16 Maximum angle difference between the SciBar and MRD tracks allowed for matching as a function of the number of steel plates. . . . . . . . . . . . 118

A.17 Schematic drawing of the matching between a SciBar track and hits in the MRD. . . . . . . . . . . . . . . . . . . . 119

B.1 Length and angle of the second track in $Q^{2}<0.15 \mathrm{GeV} / c^{2}$ in the $\mu+p$ sample. . . . . . . . . . . . . . . . . . . . 120

B.2 Vertex distributions for the data-excess enriched sample. . . . . . . . . 121

B.3 $d E / d x$ of the first (left) and second (right) tracks for the data-excess enriched sample. . . . . . . . . . . . . . . . . . . . . 121

B.4 Vertex activity for the data-excess enriched sample. . . . . . . . . . . . . 122

C.1 Muon momentum and angle distributions for the one track events. . . . . . 123

C.2 Muon momentum and angle, 2nd track length, and 2nd track angle distributions for the $\mu+p$ events. . . . . . . . . . . . . . . . . . 124

C.3 Muon momentum and angle, 2nd track length, and 2nd track angle distributions for the $\mu+\pi$ events with vertex activity. . . . . . . . . . . . . . 124

C.4 Muon momentum and angle, 2nd track length, and 2nd track angle distributions for the $\mu+\pi$ events without vertex activity. . . . . . . . . . . 125 


\section{Bibliography}

[1] Z. Maki, M. Nakagawa and S. Sakata, Prog. Theor. Phys. 28, 870 (1962).

[2] T2K, Y. Itow et al., hep-ex/0106019.

[3] S. J. Barish et al., Phys. Rev. D16, 3103 (1977).

[4] S. Bonetti et al., Nuovo Cim. A38, 260 (1977).

[5] Gargamelle Neutrino Propane, S. Ciampolillo et al., Phys. Lett. B84, 281 (1979).

[6] N. Armenise et al., Nucl. Phys. B152, 365 (1979).

[7] S. V. Belikov et al., Z. Phys. A320, 625 (1985).

[8] G. M. Radecky et al., Phys. Rev. D25, 1161 (1982).

[9] T. Kitagaki et al., Phys. Rev. D34, 2554 (1986).

[10] P. S. Auchincloss et al., Z. Phys. C48, 411 (1990).

[11] J. P. Berge et al., Z. Phys. C35, 443 (1987).

[12] V. B. Anikeev et al., Z. Phys. C70, 39 (1996).

[13] A. I. Mukhin et al., Sov. J. Nucl. Phys. 30, 528 (1979).

[14] D. MacFarlane et al., Z. Phys. C26, 1 (1984).

[15] N. J. Baker et al., Phys. Rev. D25, 617 (1982).

[16] K. Hiraide, Master's thesis, Kyoto University (2005).

[17] J. Campbell et al., Phys. Rev. Lett. 30, 335 (1973).

[18] S. J. Barish et al., Phys. Rev. D19, 2521 (1979).

[19] W. Lerche et al., Phys. Lett. B78, 510 (1978).

[20] T. Bolognese, J. P. Engel, J. L. Guyonnet and J. L. Riester, Phys. Lett. B81, 393 (1979).

[21] J. Bell et al., Phys. Rev. Lett. 41, 1008 (1978).

[22] J. Bell et al., Phys. Rev. Lett. 41, 1012 (1978). 
[23] Aachen-Bonn-CERN-Munich-Oxford, P. Allen et al., Nucl. Phys. B176, 269 (1980).

[24] Aachen-Birmingham-Bonn-CERN-London-Munich-Oxford, P. Allen et al., Nucl. Phys. B264, 221 (1986).

[25] D. Allasia et al., Nucl. Phys. B343, 285 (1990).

[26] V. V. Ammosov et al., Sov. J. Nucl. Phys. 50, 67 (1989).

[27] SKAT, H. J. Grabosch et al., Z. Phys. C41, 527 (1989).

[28] K2K, A. Rodriguez et al., Phys. Rev. D78, 032003 (2008), [0805.0186].

[29] S. L. Adler, Phys. Rev. 135, B963 (1964).

[30] S. S. Gershtein, Y. Y. Komachenko and M. Y. Khlopov, Sov. J. Nucl. Phys. 32, 861 (1980).

[31] D. Rein and L. M. Sehgal, Nucl. Phys. B223, 29 (1983).

[32] A. A. Belkov and B. Z. Kopeliovich, Sov. J. Nucl. Phys. 46, 499 (1987).

[33] E. A. Paschos, A. Kartavtsev and G. J. Gounaris, Phys. Rev. D74, 054007 (2006), [hep-ph/0512139].

[34] D. Rein and L. M. Sehgal, Phys. Lett. B657, 207 (2007), [hep-ph/0606185].

[35] S. K. Singh, M. Sajjad Athar and S. Ahmad, Phys. Rev. Lett. 96, 241801 (2006).

[36] L. Alvarez-Ruso, L. S. Geng, S. Hirenzaki and M. J. Vicente Vacas, Phys. Rev. C75, 055501 (2007), [nucl-th/0701098].

[37] L. Alvarez-Ruso, L. S. Geng and M. J. Vicente Vacas, Phys. Rev. C76, 068501 (2007), [0707.2172].

[38] H. Faissner et al., Phys. Lett. B125, 230 (1983).

[39] E. Isiksal, D. Rein and J. G. Morfin, Phys. Rev. Lett. 52, 1096 (1984).

[40] SKAT, H. J. Grabosch et al., Zeit. Phys. C31, 203 (1986).

[41] CHARM, F. Bergsma et al., Phys. Lett. B157, 469 (1985).

[42] CHARM-II, P. Vilain et al., Phys. Lett. B313, 267 (1993).

[43] BEBC WA59, P. Marage et al., Z. Phys. C31, 191 (1986).

[44] BEBC WA59, P. P. Allport et al., Z. Phys. C43, 523 (1989).

[45] E632, M. Aderholz et al., Phys. Rev. Lett. 63, 2349 (1989).

[46] E632, S. Willocq et al., Phys. Rev. D47, 2661 (1993).

[47] K2K, M. Hasegawa et al., Phys. Rev. Lett. 95, 252301 (2005), [hep-ex/0506008]. 
[48] MiniBoonE, A. A. Aguilar-Arevalo et al., Phys. Lett. B664, 41 (2008), [0803.3423].

[49] SciBooNE, A. A. Aguilar-Arevalo et al., hep-ex/0601022.

[50] K. Nitta et al., Nucl. Instrum. Meth. A535, 147 (2004), [hep-ex/0406023].

[51] SciBooNE, K. Hiraide, Nucl. Phys. Proc. Suppl. 159, 85 (2006).

[52] SciBooNE, K. Hiraide, AIP Conf. Proc. 967, 316 (2007).

[53] SciBooNE, K. Hiraide, AIP Conf. Proc. 981, 253 (2008).

[54] MiniBooNE, A. A. Aguilar-Arevalo et al., 0806.1449.

[55] GEANT4, S. Agostinelli et al., Nucl. Instrum. Meth. A506, 250 (2003).

[56] M. G. Catanesi et al., Eur. Phys. J. C52, 29 (2007), [hep-ex/0702024].

[57] E910, I. Chemakin et al., Phys. Rev. C77, 015209 (2008), [0707.2375].

[58] J. R. Sanford and C. L. Wang, BNL Note No. 11299 (1967).

[59] N. V. Mokhov et al., nucl-th/9812038.

[60] Y. Hayato, Nucl. Phys. Proc. Suppl. 112, 171 (2002).

[61] G. Mitsuka, AIP Conf. Proc. 981, 262 (2008).

[62] C. H. Llewellyn Smith, Phys. Rept. 3, 261 (1972).

[63] K2K, R. Gran et al., Phys. Rev. D74, 052002 (2006), [hep-ex/0603034].

[64] MiniBooNE, A. A. Aguilar-Arevalo et al., Phys. Rev. Lett. 100, 032301 (2008), [0706.0926].

[65] L. A. Ahrens et al., Phys. Rev. Lett. 56, 1107 (1986).

[66] C. H. Albright, C. Quigg, R. E. Shrock and J. Smith, Phys. Rev. D14, 1780 (1976).

[67] R. A. Smith and E. J. Moniz, Nucl. Phys. B43, 605 (1972).

[68] D. Rein and L. M. Sehgal, Ann. Phys. 133, 79 (1981).

[69] R. P. Feynman, M. Kislinger and F. Ravndal, Phys. Rev. D3, 2706 (1971).

[70] C. Berger and L. M. Sehgal, Phys. Rev. D76, 113004 (2007), [0709.4378].

[71] K. S. Kuzmin, V. V. Lyubushkin and V. A. Naumov, Mod. Phys. Lett. A19, 2815 (2004), [hep-ph/0312107].

[72] D. Rein, Z. Phys. C35, 43 (1987).

[73] S. K. Singh, M. J. Vicente-Vacas and E. Oset, Phys. Lett. B416, 23 (1998).

[74] M. Gluck, E. Reya and A. Vogt, Eur. Phys. J. C5, 461 (1998), [hep-ph/9806404]. 
[75] A. Bodek and U. K. Yang, hep-ex/0308007.

[76] KAMIOKANDE, M. Nakahata et al., J. Phys. Soc. Jap. 55, 3786 (1986).

[77] M. Derrick et al., Phys. Rev. D17, 1 (1978).

[78] Z. Koba, H. B. Nielsen and P. Olesen, Nucl. Phys. B40, 317 (1972).

[79] Amsterdam-Bologna-Padua-Pisa-Saclay-Turin, S. Barlag et al., Zeit. Phys. C11, 283 (1982).

[80] T. Sjostrand, Comput. Phys. Commun. 82, 74 (1994).

[81] P. Musset and J. P. Vialle, Phys. Rept. 39, 1 (1978).

[82] J. E. Kim, P. Langacker, M. Levine and H. H. Williams, Rev. Mod. Phys. 53, 211 (1981).

[83] C. W. De Jager, H. De Vries and C. De Vries, Atom. Data Nucl. Data Tabl. 14, 479 (1974).

[84] L. L. Salcedo, E. Oset, M. J. Vicente-Vacas and C. Garcia-Recio, Nucl. Phys. A484, 557 (1988).

[85] D. Ashery et al., Phys. Rev. C23, 2173 (1981).

[86] G. Rowe, M. Salomon and R. H. Landau, Phys. Rev. C18, 584 (1978).

[87] H. W. Bertini, Phys. Rev. C6, 631 (1972).

[88] S. J. Lindenbaum and R. M. Sternheimer, Phys. Rev. 105, 1874 (1957).

[89] J. Birks, Theory and Practice of Scintillation Counting (Pergamon Press, 1964).

[90] M. Hasegawa, Ph.D. thesis, Kyoto University (2006).

[91] P. Adamson et al., Nucl. Instrum. Meth. A492, 325 (2002), [hep-ex/0204021].

[92] T. Morita, Master's thesis, Kyoto University (2004).

[93] T. Sasaki, Master's thesis, Kyoto University (2004).

[94] S. Yamamoto, Master's thesis, Kyoto University (2003).

[95] M. Yoshida et al., IEEE Trans. Nucl. Sci. 51, 3043 (2004).

[96] Y. Arai et al., IEEE Trans. Nucl. Sci. 49, 1164 (2002).

[97] S. Buontempo et al., Nucl. Instrum. Meth. A349, 70 (1994).

[98] J. A. Crittenden et al., Phys. Rev. D34, 2584 (1986).

[99] A. Heikkinen, N. Stepanov and J. P. Wellisch, nucl-th/0306008.

[100] C. Wilkin et al., Nucl. Phys. B62, 61 (1973). 
[101] A. S. Clough et al., Nucl. Phys. B76, 15 (1974).

[102] A. S. Carroll et al., Phys. Rev. C14, 635 (1976).

[103] R. D. Ransome et al., Phys. Rev. C45, 509 (1992).

[104] M. K. Jones et al., Phys. Rev. C48, 2800 (1993).

[105] H. Maesaka, Ph.D. thesis, Kyoto University (2005).

[106] S. Baker and R. D. Cousins, Nucl. Instrum. Meth. 221, 437 (1984).

[107] F. James and M. Roos, Comput. Phys. Commun. 10, 343 (1975).

[108] E. J. Moniz et al., Phys. Rev. Lett. 26, 445 (1971).

[109] V. Bernard, L. Elouadrhiri and U. G. Meissner, J. Phys. G28, R1 (2002), [hepph/0107088].

[110] SciBooNE, K. Hiraide et al., Phys. Rev. D78, 112004 (2008), [0811.0369].

[111] J. E. Amaro, E. Hernandez, J. Nieves and M. Valverde, 0811.1421.

[112] C. Berger and L. M. Sehgal, 0812.2653.

[113] Minerva, D. Drakoulakos et al., hep-ex/0405002.

[114] Y. Kurimoto, The SciBooNE Experiment at FNAL, in Neutrino Oscillation Workshop, Otranto, Lecce, Italy, 2008.

[115] W. Stephen, Cellular Automata and Complexity (Westview Press, 2002). 